\title{
ANAL YTICAL AND EXPERIMENTAL INVESTIGATION OF THE EFFECTS OF THE MACHINING PROCESSES ON THE MECHANICAL BEHAVIOUR OF CARBON EPOXY COMPOSITE LAMINATES
}

\author{
by \\ Muhammad Saleem \\ Bachelor of Engineering in Mechanical Engineering \\ NWFP University of Engineering \& Technology, Peshawar, Pakistan, 1991 \\ Master of Applied Science, \\ Ryerson University, Toronto, Canada, 2010
}

A dissertation presented to

RYERSON UNIVERSITY

in partial fulfillment of the

requirements for the degree of

DOCTOR OF PHILOSOPHY

in the program of

MECHANICAL \& INDUSTRIAL ENGINEERING

Toronto, Ontario, Canada, 2014

(C) Muhammad Saleem, 2014 


\section{AUTHOR'S DECLARATION FOR ELECTRONIC SUBMISSION OF A DISSERTATION}

I hereby declare that I am the sole author of this dissertation. This is a true copy of the dissertation, including any required final revisions, as accepted by my examiners.

I authorize Ryerson University to lend this dissertation to other institutions or individuals for the purpose of scholarly research.

I further authorize Ryerson University to reproduce this dissertation by photocopying or by other means, in total or in part, at the request of other institutions or individuals for the purpose of scholarly research.

I understand that my dissertation may be made electronically available to the public. 


\title{
ANALYTICAL AND EXPERIMENTAL INVESTIGATION OF THE EFFECTS OF THE MACHINING PROCESSES ON THE MECHANICAL BEHAVIOUR OF CARBON EPOXY COMPOSITE LAMINATES
}

\author{
Muhammad Saleem
}

\section{Doctor of Philosophy, Mechanical \& Industrial Engineering, Ryerson University, Toronto} (2014)

\begin{abstract}
To join various components, drilling is the most frequently used machining process for carbon fiber-reinforced polymer (CFRP) composites. However, it induces various defects such as microcracks, resin degradation and fiber pull-out. In order to eliminate these problems, an appropriate machining process must be employed. Therefore, the main objective of this research is to conduct an analytical and experimental study to investigate the influence of the machining process on the mechanical behaviour of a CFRP structural component and assembly drilled with conventional (CM) and abrasive water-jet machining (AWJM) processes.

All CFRP composite laminates did not demonstrate any prominent change in the mechanical properties during static tests. However, fatigue tests showed that the damage accumulation in conventional machining was higher than that in AWJM specimens. Thus, in the case of CM specimens the endurance limit was less than AWJM specimens. The difference in the mechanical behaviour of the composite laminates can be related to the initial surface integrity induced by the difference in the mechanism of material removal of each machining process. This difference in surface texture was responsible for the initiation of stress concentration sites as evident from infrared thermographic stress and thermal analysis. The heat dissipated from all laminates was also correlated to the damage accumulation. It was identified that the surface roughness criterion ( $R a$ ) used for the characterization of the surface roughness for metals was not suitable for composites as roughness values of all specimens were same, however different mechanical behaviour was observed. An IR thermographic damage criterion (TDC) was developed which related the temperature threshold area with damage evolution. TDC further confirms the
\end{abstract}


superiority of AWJM compared to CM process. Further it was identified that AWJM process was less sensitive to the composite's stacking sequence compared to conventional machining. The main conclusion of this research is that the failure modes of composite structure parts and assembly are highly affected by the choice of the machining process. This study therefore confirms that the machining process has an important contribution for the design of composite parts and assembly in order to improve the service life of the machined composite structures. 


\section{Acknowledgements}

I am highly indebted to my colleagues, friends and instructors who provided me all the help; I have needed throughout my $\mathrm{PhD}$ studies and the completion of this dissertation. First, I wish to express my deep sense of gratitude to my supervisor Dr. Habiba Bougherara for her continuous encouragement, guidance and support throughout my studies, including the writing of this dissertation. I am also thankful to my supervisor for providing me the opportunity to work on this research project and to present my work in a number of international conferences. In addition, Dr. Habiba Bougherara must be credited for sparking my interest in research and also has been a wonderful influence on my career. I am thankful for her much-needed encouragement that helped me see this dissertation through.

I am also thankful for the support of Dr. Lotfi Toubal (University of Quebec at Trois-Rivieres Canada) and Dr. Redouane Zitoune (Institut Clément Ader France), for their collaboration and invaluable technical knowledge for the completion of this project. I would also like to thank Dr. Redouane Zitoune for providing composite material for testing. I express sincere thanks to JEDO TECHNOLOGIE industry, Toulouse, French for providing specimens drilled with water-jet machining process. Mr. Ihab provided his precious time and expertise in the experimental work of infrared thermography, and fatigue testing. Mr. Ihab provided me with useful input that enriched my understanding and enthusiasm on the field. Last, but certainly not least, I recognize my parents and wife for their selfless and absolute support in good times and bad.

Muhammad Saleem

Toronto, August 2014 


\section{TABLE OF CONTENTS}

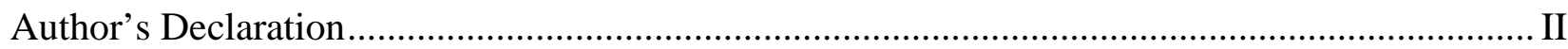

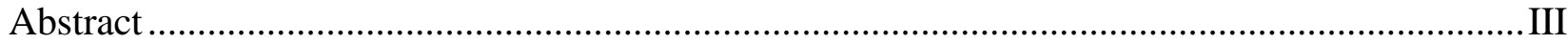

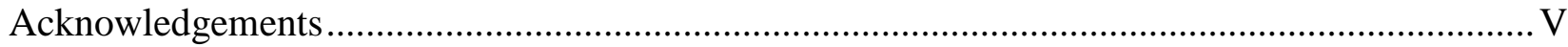

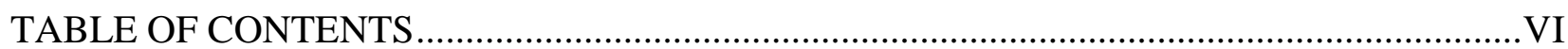

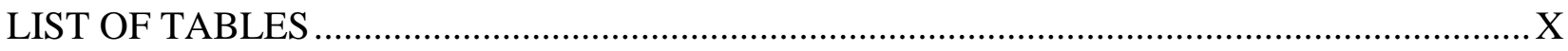

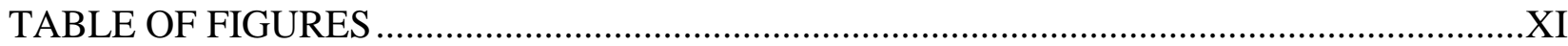

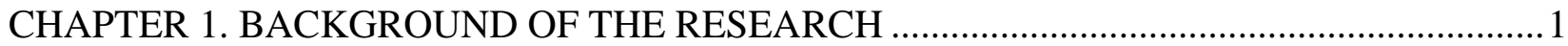

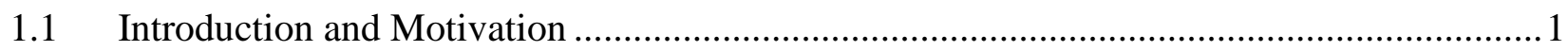

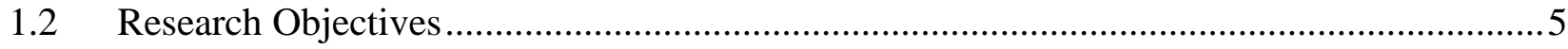

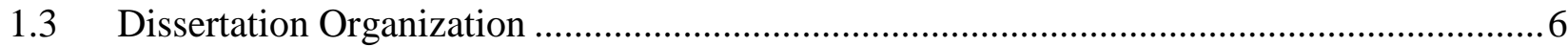

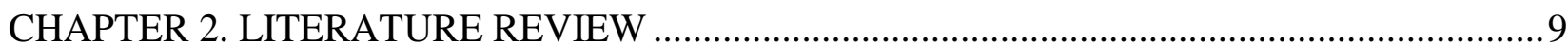

2.1 Conventional Machining of Composite Material ...............................................................

2.1.1 Parametric Study ……………………………...............................................10

2.1.2 Effect of Drilling Parameters on the Thrust Force..................................................11

2.1.3 Effect of cutting Parameters on Delamination .......................................................11

2.1.4 Effect of the Cutting Tool on the Hole Geometry..................................................12

2.2 Effect of a machined Surface on Stress Concentration.....................................................13

2.3 Fatigue Behaviour of Composite Plates..............................................................................13

2.4 Challenges in the drilling of Composites............................................................................

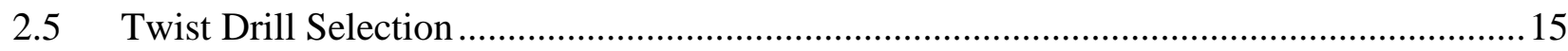

2.6 Non-Conventional machining of Composite Laminates.......................................................16

2.6.1 Selection of a non-conventional machining process .............................................17

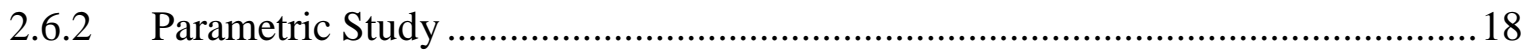


Standoff distance .............................................................18

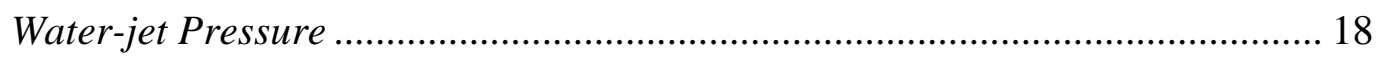

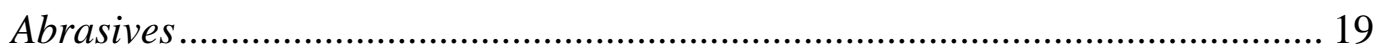

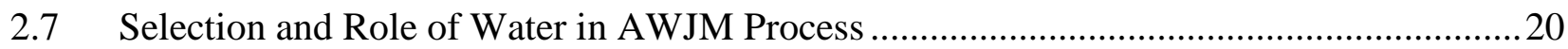

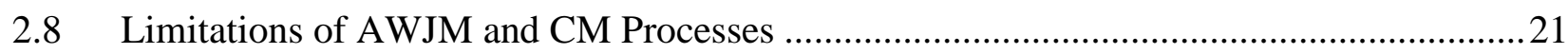

2.9 Effect of Machining Process on the Surface Roughness ....................................................21

2.9.1 Effect of CM Process on Surface Roughness........................................................22

2.9.2 Effect of AWJM Process on Surface Roughness ...................................................24

2.10 Fatigue Effect on Composite Structure..........................................................................2

2.10.1 Fatigue behaviour of the Machined Surface …………..........................................26

2.11 Fatigue Failure Mechanism.......................................................................................27

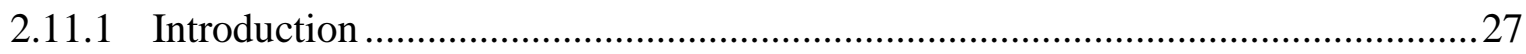

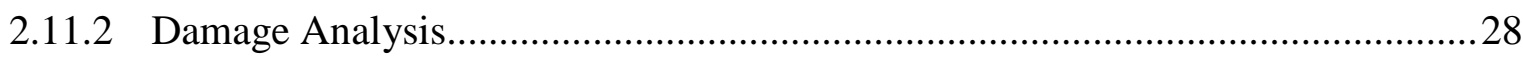

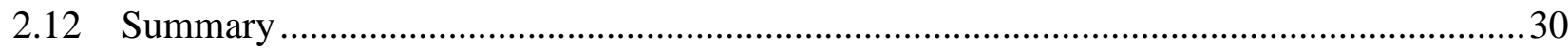

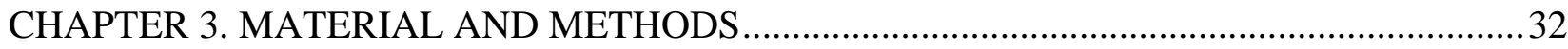

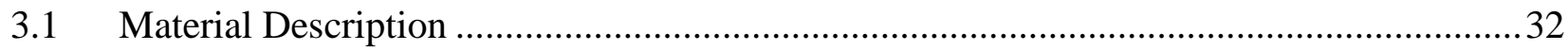

3.2 Specimen Preparation Details ...........................................................................................3

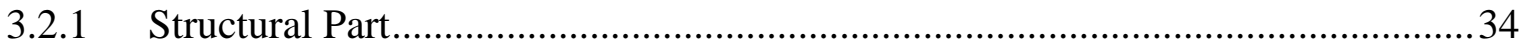

3.2.2 Structural Assembly ............................................................................................. 35

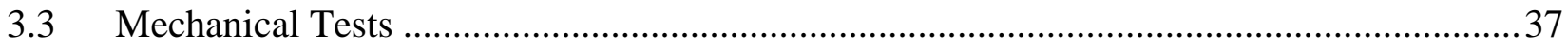

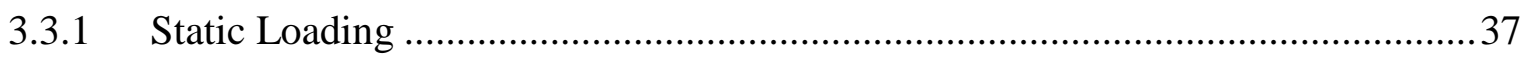

3.3.2 Fatigue Loading................................................................................................. 38

3.4 Infrared Thermographic Measurement ………............................................................... 40

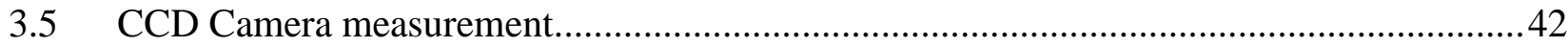




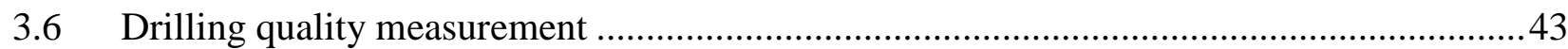

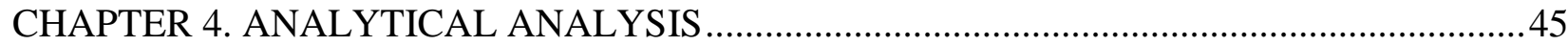

4.1 Analytical stress and strains in orthotropic laminate ........................................................45

4.1.1 Calculations for Elastic properties of Composite Laminate ……….......................46

4.1.2 Hook's law for orthotropic composite laminate ....................................................48

4.1.3 Stress-strain relations for orthotropic lamina ........................................................50

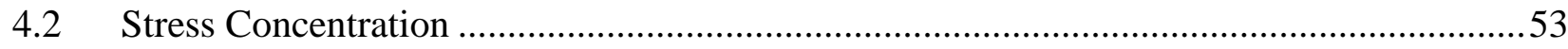

4.2.1 Stress Concentration Criteria ……………………………………………….....54

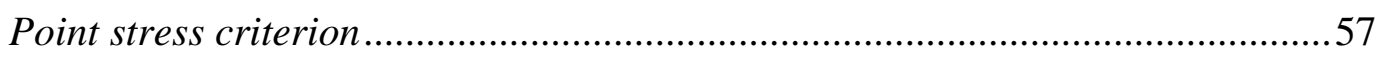

Average stress criterion ................................................................................. 58

4.2.2 IR Thermal and Stress concentration factor .........................................................58

4.3 Stress field at and far from the circular hole .......................................................................61

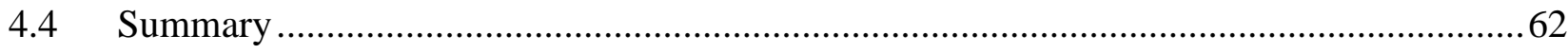

CHAPTER 5. STATIC TEST RESULTS FOR THE SINGLE PLATE ........................................64

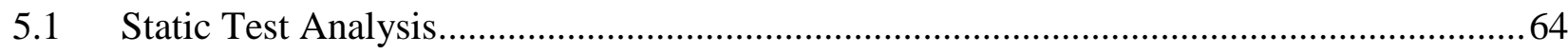

5.1.1 Results and discussion...................................................................................64

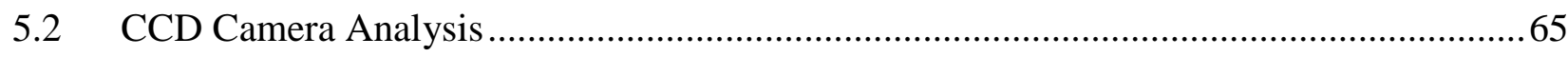

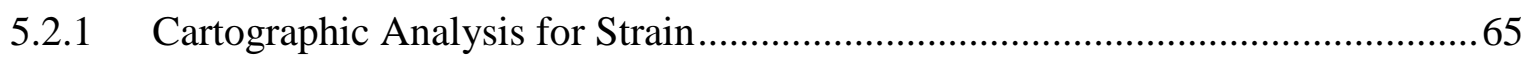

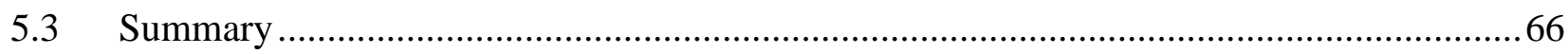

CHAPTER 6. FATIGUE TEST RESULTS FOR THE SINGLE PLATE ………...........................70

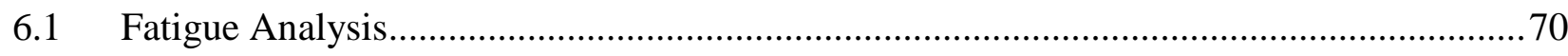

6.1.1 Elastic Modulus Results and Discussion............................................................70

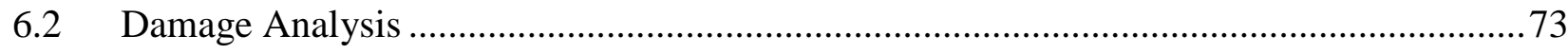

6.2.1 Damage assessment (Angle ply Laminate) ………………....................................73 
6.2.2 Fatigue damage assessment of Angle ply and quasi isotropic lay-ups ................81

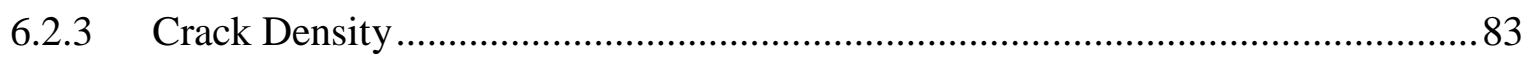

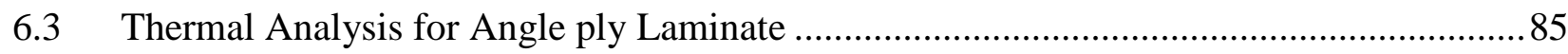

6.4 Damage assessment of angle ply and quasi isotropic laminates using IR thermographic...90

6.5 Correlation between Heat Dissipation and Damage accumulation...................................92

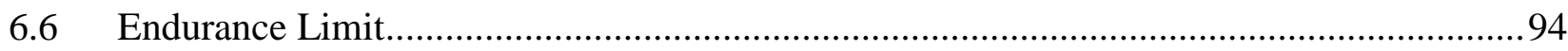

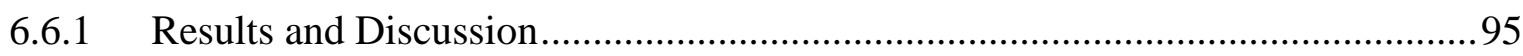

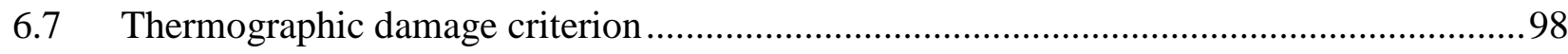

6.8 Surface Morphology of the Post-fatigued Angle ply Laminate.................................... 102

6.9 Surface Morphology of the Post-fatigued Angle ply and Quasi Isotropic laminates......... 106

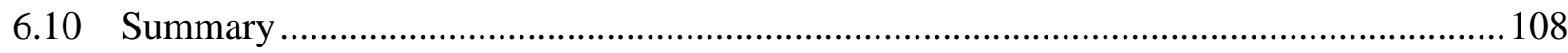

CHAPTER 7. FATIGUE TESTING OF CFRP ASSEMBLY …........................................112

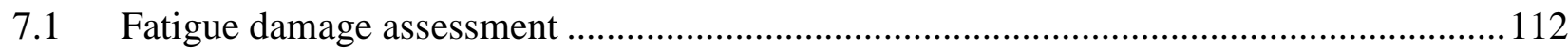

7.2 Damage assessment using infrared thermography................................................... 120

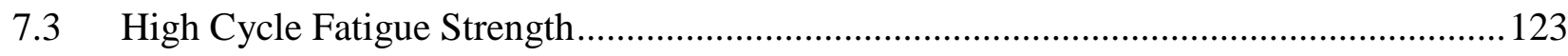

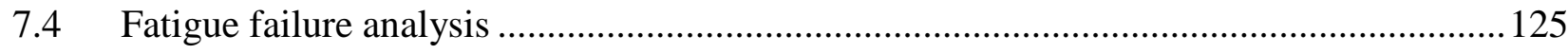

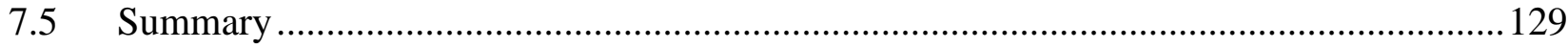

CHAPTER 8. CONCLUSION, CONTRIBUTIONS AND FUTURE WORK.........................130

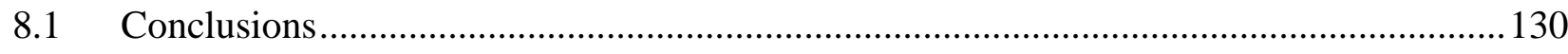

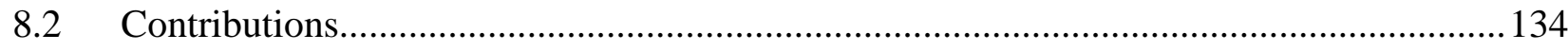

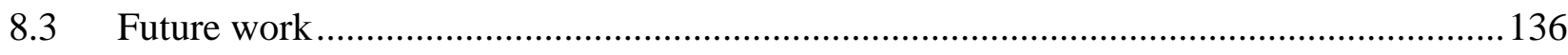

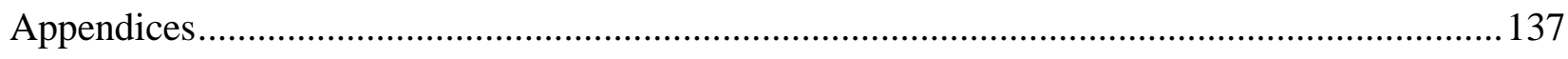

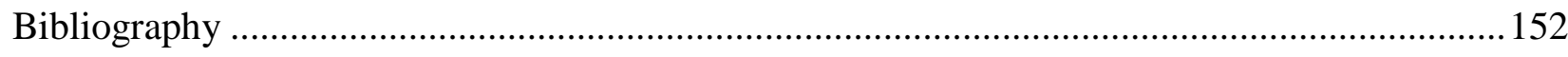




\section{LIST OF TABLES}

Table 2-1 Significance of AWJ parameters on machining results [79] .................................. 19

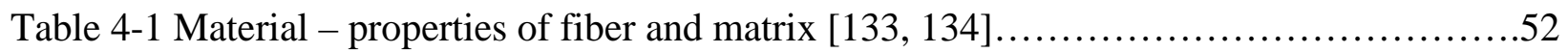

Table 4-2 Calculated lamina and laminate material properties from MATLAB code ................ 53

Table 4-3 Effect of machining process on the stress concentration factors and strength of carbon/epoxy composite with circular hole under uniaxial tensile loading............................... 60

Table 6-1 illustrated functional performance and roughness parameters relation. A solid circle shows a significant amount of information linking the surface finish to the functional performance category, a half solid circle shows that there is some evidence and an open circle

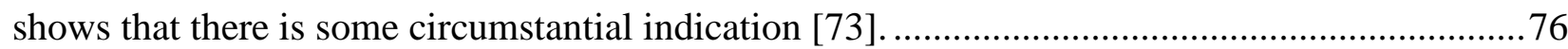

Table 6-2 2D Statistical roughness parameters of average surface roughness $R a$, maximum profile valley depth $R v$ and skewness Rsk for the two types of specimens, All values were

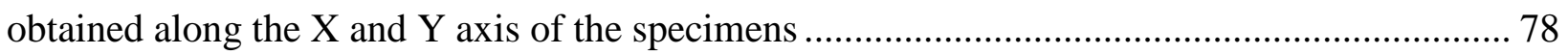

Table 6-3 Surface roughness of pre-fatigue CF/Epoxy assembly specimens drilled with the CM

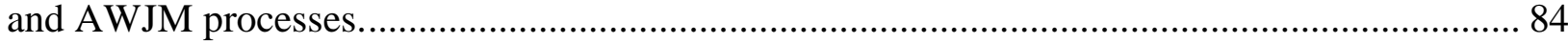

Table 6-4 The \% area of crack of angle ply and quasi isotropic CF/Epoxy laminates machined with (a) CM and (b) AWJM technique, pre-fatigue testing .................................................. 85

Table 7-1 Surface roughness of pre-fatigue CF/Epoxy assembly specimens drilled with CM and AWJM processes..................................................................118

Table 7-2 The \% area of crack of CF/Epoxy pre-fatigue specimens drilled with CM and AWJM processes. Crack density was calculated using image analysis software. 118 


\section{TABLE OF FIGURES}

Figure 2-1 Terminology of a standard point twist drill [66] ..............................16

Figure 2-2 Links between surface features and performance [73] ......................................... 23

Figure 2-3 Surface roughness $(R a)$ versus feed rate and for various spindle speed for CFRP and

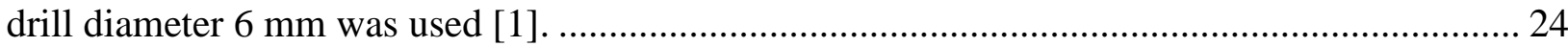

Figure 2-4 The evolution of the modulus of elasticity E vs damage D according to the number of

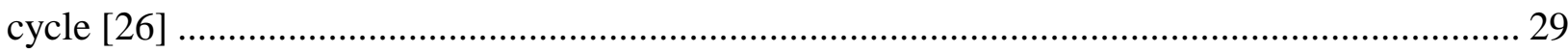

Figure 3-1 Unidirectional fibers used for manufacturing of composite laminates................33

Figure 3-2 Twist drill used for conventional machining ................................................................ 34

Figure 3-3 Composite plate with centre hole drilled with conventional and abrasive water-jet machining processes, used for static and fatigue testing .............................................................. 34

Figure 3-4. Geometry of single-lap, single-bolt and single-shear assembly manufactured from two pieces of CF/Epoxy composite laminates ................................................................................ 36

Figure 3-5 (a) Experimental set-ups for fatigue loading for the composite plate, IR camera was positioned at about $100 \mathrm{~cm}$ from the specimen, (b) photograph of extensometer instrumented on the composite material specimen. 39

Figure 3-6 Loading pattern of carbon epoxy laminates, increasing loading stages from $3 \mathrm{kN}$ to $11 \mathrm{kN}$ at 5000 cycles for each fatigue loading stage [120]........................................................... 40

Figure 3-7 Temperature variations during fatigue loading [26] .................................................. 42

Figure 3-8 Experimental devices for static and fatigue tests of assembly specimens, showing infrared camera and assembly specimen ................................................................................. 42

Figure 3-9 Surface Roughness test using NANOVEA 400 series tester ....................................... 43

Figure $4-1 \mathrm{x}$ and $\mathrm{y}$ shows global while 1 and 2 represent the local coordinate system...................46

Figure 4-2 Stress components in global x, y, z coordinate system ............................................... 49

Figure 4-3 Geometry of multilayer composite laminate................................................................ 51

Figure 4-4 orthotropic lamina showing fiber angle, global to local coordinate system ................ 52 
Figure 4-5 Orthotropic composite plate with circular hole.

Figure 4-6 Failure mechanism of the circular hole specimen representing point and average stress criterion 55

Figure 4-7 Stress distribution of CFRP composites with circular hole subjected to a tensile load along long axis of the specimen. 62

Figure 5-1 Static tests strain values from stress vs. strain graph for the laminates drilled with abrasive water-jet and conventional machining process.

Figure 5-2 Digital images showing: (a) strain coordinates, and specimen showing cartography of the deformations in plan YY $\left(\varepsilon_{\mathrm{yy}}\right)$, (b) XX $\left(\varepsilon_{\mathrm{xx}}\right)$ and (c) XY $\left(\varepsilon_{\mathrm{xy}}\right)$ under axial tensile load of 14 $\mathrm{KN}$ in the direction of OY for CM specimen 67

Figure 5-3 Digital images showing deformations in plans (a) YY ( $\left.\varepsilon_{\mathrm{yy}}\right)$, (b) XX $\left(\varepsilon_{\mathrm{xx}}\right)$ and (c) XY $\left(\varepsilon_{\mathrm{xy}}\right)$ under axial tensile load of $14 \mathrm{KN}$ in the direction of OY for AWJM specimen. 68 Figure 6-1 Calculated experimental tests results of the elastic modulus vs. normalized cycles for fatigue loading from $3 \mathrm{kN}$ to rupture of all (a) CM and (b) AWJM specimens. All units are in Megapascal. .71

Figure 6-2 Calculated experimental tests results of the elastic modulus vs. normalized cycles for fatigue loading cases of (a) 53\% and (b) 59\% load to rupture of all specimens. A similar trend was also obtained for all other fatigue loading cases of specimens drilled with conventional and AWJ machining. All units are in Megapascal. 72

Figure 6-3 Three regions of cracking propagating mechanism in composite laminate [139] ...... 72

Figure 6-4 Comparison of the evolution of the damage for various level of loading for (a) Conventional machining and (b) Abrasive water-jet machining..... 73

Figure 6-5 Comparison of the evolution of the damage for fatigue loading cases of (a) 59\% and for (b) $65 \%$ load to rupture, for all examined specimens. 74

Figure 6-6 Cartography of the surface roughness of the wall of the hole for different specimens: (a) hole obtained with conventional machining using a cutting tool and (b) with abrasive waterjet. 77 
Figure 6-7 SEM photographs comparing the hole surfaces machined with two different techniques. A circular hole was machined with (a) conventional cutting tool and (b) Abrasive water-jet. 80

Figure 6-8 The evolution of damage for various level of for CF/Epoxy quasi isotropic specimens machined with (a) CM and (b) AWJM technique, tested at 10Hz frequency and 10000 cycles.. 81 Figure 6-9 The evolution of damage for various level of loading for CF/Epoxy quasi isotropic specimens machined with (a) CM (b) AWJM technique, tested at $10 \mathrm{~Hz}$ frequency and 10000 cycles. 82

Figure 6-10 Surface Roughness images showing the cross section of circular hole of CF/Epoxy angle ply lay-up laminates, pre-fatigue test, a circular hole obtained with the (a) CM (b) AWJM techniques. 83

Figure 6-11 Surface Roughness images showing the cross section of circular hole of quasi isotropic lay-up laminate, pre-fatigue test, a circular hole obtained with the (a) CM and (b) AWJM techniques 84

Figure 6-12 Image processing step by step procedure to determine the damage region. 85 Figure 6-13 Stages of the temperature changes for fatigue loading. In each stage, a load was applied for 5000 cycles $(1250 \times 4)$ till rupture for the CM specimen. A similar temperature trend was also obtained for all the CM specimens. 86

Figure 6-14 Stages of the temperature changes for fatigue loading. In each stage a load was applied for 5000 cycles $(1250 \mathrm{x}$ 4) till rupture for the AWJM specimen. A similar temperature trend was also obtained for all AWJM specimens. 87 Figure 6-15 Temperature contours for the CM specimens at 5000 cycles under (a) $3 \mathrm{kN}$, (b) $8 \mathrm{kN}$ and (c) $10 \mathrm{kN}$ cyclic loads. The area around the hole within the rectangle is considered for investigation. 88 Figure 6-16 Temperature contours for AWJM specimens at 5000 cycles under (a) 3 kN, (b) 8 kN and (c) $11 \mathrm{kN}$ cyclic loads. The highest temperatures are in pink/violet and the lowest are in black/dark blue. The area around the hole within the rectangle is considered for investigation. 88 Figure 6-17 Temperature maps for loads (a) $3 \mathrm{kN}$, (b) $5 \mathrm{kN}$ (c) $7 \mathrm{kN}$ and (d) $8 \mathrm{kN}$ of angle ply 90 
Figure 6-18 Temperature maps for loads (a) $3 \mathrm{kN}$, (b) $5 \mathrm{kN}$ (c) $7 \mathrm{kN}$ and (d) $9 \mathrm{kN}$ of angle ply AWJM specimen at $10 \mathrm{~Hz}$ frequency and 10000 cycles.

Figure 6-19 Temperature maps for loads (a) 20\%, (b) 55\% and (c) and (d) $65 \%$ of quasi isotropic CM specimen at $10 \mathrm{~Hz}$ frequency and 10000 cycles. 91

Figure 6-20 Temperature maps for loads (a) 20\%, (b) 55\% and (c) and (d) $70 \%$ of quasi isotropic AWJM specimen at $10 \mathrm{~Hz}$ frequency and 10000 cycles. 92

Figure 6-21 Comparison between the change of the maximum temperature and the damage for AWJM specimen at a different level of loading: $6 \mathrm{kN}, 8 \mathrm{kN}$ and $10 \mathrm{kN}$...................................... 94

Figure 6-22 Endurance limit prediction [122] ....................................................................... 95

Figure 6-23 Graphical representation of endurance limits for the specimens (a) CM and (b) AWJM. The endurance limit obtained by intersecting the two straight lines that interpolate the experimental data of the stress and temperature. 96

Figure 6-24 The endurance limit of CF/Epoxy specimens machined with (a)(c) AWJM and (b)(d) $\mathrm{CM}$ processes, tested at $10 \mathrm{~Hz}$ frequency and at 10000 cycles. .................................................... 97

Figure 6-25 Cumulative damage zone under increasing fatigue cycles [148]............................. 99

Figure 6-26 shows the thermal threshold area considered in Equation (47) for evaluating the damage 100

Figure 6-27 Infrared thermographic image showing thermal threshold area 100

Figure 6-28 Comparison of the area of the portion having maximum temperature with the each loading stage from $3 \mathrm{kN}$ to $11 \mathrm{kN}$. 101

Figure 6-29 Comparison of the maximum and mean temperature against applied load for CM and AWJM specimens 102

Figure 6-30 SEM micrograph showing a cross section of the circular hole of CF/Epoxy angle ply composite, circular hole obtained with the CM. 103

Figure 6-31 Fractured carbon epoxy composite laminate. 104

Figure 6-32 SEM micrograph showing cross section of circular hole of CF/Epoxy angle ply composite, circular hole obtained with AWJM 105 
Figure 6-33 SEM micrograph showing the cross section of a circular hole of CF/Epoxy angle ply (Fig. a and b) and quasi isotropic (Fig. c and d) laminates, the circular hole was obtained with the CM process 107

Figure 6-34 SEM micrograph showing cross section of circular hole of CF/Epoxy angle ply (Fig. a) and quasi isotropic (Fig. b) laminates, circular hole obtained with AWJM process 107

Figure 7-1. The evolution of the damage for various level of loading for CF/Epoxy quasi isotropic assembly specimens. Specimens machined with (a): the CM process and (b): the AWJM process. Condition of the tests: frequency of $10 \mathrm{~Hz}$ and number of the cycles for each level of loading is 10000 113

Figure 7-2 The evolution of the damage for various level of loading for CF/Epoxy angle ply assembly specimens. Specimens machined with (a): the CM process and (b): the AWJM process. Condition of the tests: frequency of $10 \mathrm{~Hz}$ and number of the cycles for each level of loading is 10000.

Figure 7-3 Surface Roughness images showing cross section of circular hole of pre-fatigue Carbon/Epoxy quasi isotropic laminate, circular hole obtained with (a) CM and (b) AWJM processes, while Figure (a_1) and (b_1) shows SEM images of the selected locations. 116

Figure 7-4 Surface Roughness images showing angle ply of circular hole of pre-fatigue Carbon/Epoxy angle ply lay-up laminate, circular hole obtained with (a) CM (b) AWJM processes

Figure 7-5 Hole elongation during fatigue testing of AWJM angle ply lay-up assembly specimen for loads of (a) $65 \%$ (b) $65 \%$ (c) $70 \%$ (d) $75 \%$ (e) $80 \%$ of UTS. 119 Figure 7-6 Hole elongation during fatigue testing of AWJM quasi isotropic assembly specimen for loads of (a) $65 \%$ (b) $70 \%$ (c) $75 \%$ (d) $80 \%$ (e) $85 \%$ of UTS. 119 Figure 7-7 Temperature maps for loads of (a) $25 \%$, (b) $45 \%$ (c) $65 \%$ and (d) $80 \%$ of AWJM angle ply lay-up assembly specimen tested at $10 \mathrm{~Hz}$ frequency and 10000 cycles. 120 Figure 7-8 Temperature maps for loads of (a) 25\% (b) $45 \%$ (c) $65 \%$ and (d) $70 \%$ for CM angle ply lay-up assembly specimen tested at $10 \mathrm{~Hz}$ frequency and 10000 cycles...... 121 
Figure 7-9 Temperature maps for loads of (a) $25 \%$ (b) $45 \%$ (c) $65 \%$ and (d) $85 \%$ for AWJM quasi isotropic lay-up assembly specimen tested at $10 \mathrm{~Hz}$ frequency and 10000 cycles.

Figure 7-10 Temperature maps for loads of (a) $25 \%$ (b) $45 \%$ (c) $65 \%$ and (d) $75 \%$ for CM quasi isotropic lay-up assembly specimen tested at $10 \mathrm{~Hz}$ frequency and 10000 cycles. 122

Figure 7-11 Graphs showing correlation of damage and temperature of (a) angle ply and (b) quasi isotropic lay-up assembly specimens drilled with AWJM process

Figure 7-12 The high cycle fatigue strength of CF/Epoxy angle ply lay-up assembly specimens machined with (a) AWJM and (b) CM process, tested at 10Hz frequency at 10000 cycles. ..... 124 Figure 7-13 The high cycle fatigue strength of CF/Epoxy quasi isotropic lay-up assembly specimens machined with (a) AWJM and (b) CM technique, tested at $10 \mathrm{~Hz}$ frequency at 10000 cycles. 125

Figure 7-14 Images showing failure mode of CF/Epoxy angle ply lay-up laminates (a) IR image and (b) photograph of AWJM fractured specimen. While (c) and (d) shows IR image and photograph of CM fractured specimen, respectively 126

Figure 7-15 Images showing failure modes of CF/Epoxy quasi isotropic lay-up composite assembly. IR images (a1,b1) and (a2,b2) shows images before final failure, IR images (c1) and (c2) shows failure, while (d1) and (d2) is the photograph of fractured specimen and (e1) and e2 illustrates fractured specimens from thickness side of AWJM and CM laminates, respectively127 


\section{LIST OF APPENDICES}

Appendix A-1 Matlab code for calculating Material Properties and Stresses 137

Appendix A-2 Experimental and Calculated Strain values (Equation 20) in global and local coordinate system for +45 and -45 degree angle plies.

Appendix A-3 Calculated Stress values (from Equation 24) in local coordinate system for +45 and -45 degree angle plies 142 Appendix A-4 The elastic modulus vs normalized cycles for fatigue loading for (a) L1-CD1, (b) L2-PD1, (c) L4-PD1 and (d) L2-WJ120d specimens. 143 Appendix A-5 The elastic modulus vs normalized cycles for fatigue loading for (a) L2-WJ120, (b) L2-WJ220, (c) L4-WJ120 and (d) L4-WJ220 specimens. 144 Appendix A-6 Comparison of the evolution of the damage for specimens (a) L1-CM1, (b) L2PD1, (c) L4-PD1 and (d) L2-WJ120d. 145

Appendix A-7 Comparison of the evolution of the damage for specimens (a) L2-WJ120, (b) L2WJ220, (c) L4-WJ120 and (d) L4-WJ220. 146

Appendix A-8 Various stages of the temperature for fatigue loading cases of 18, 24, 29, 35, 41, 47, 53, and 59 \% load to rupture for (a) L1-CM, (b) L2-PD1, (c) L4-PD1, (d) L2-WJ120d specimens.

Appendix A-9 Various stages of stabilization of the temperature for fatigue loading cases of 18, 24, 29, 35, 41, 47, 53, and 59 \% load to rupture for (a) L2-WJ120, (b) L2-WJ220, (c) L4-WJ120, (d) L4-WJ220 specimens.

Appendix A-10 The evolution of the damage for various level of loading for Carbon/Epoxy (a) quasi isotropic and (b) angle ply lay-up assembly specimen tested at $10 \mathrm{~Hz}$ frequency for 10000 cycles, drilled with AWJM processes.

Appendix A-11 The endurance limit of $\left[ \pm 45^{\circ}\right]_{2 S}$ CF/Epoxy specimens machined with (a) AWJM and (b) CM technique, tested at $10 \mathrm{~Hz}$ frequency at 10000 cycles. 149 Appendix A-12 The endurance limit of [90/+45/0/-45] $]_{S}$ CF/Epoxy specimens machined with (a) AWJM and (b) CM technique, tested at $10 \mathrm{~Hz}$ frequency and at 10000 cycles. 150 
Appendix A-13, List of Publications . 


\section{CHAPTER 1. BACKGROUND OF THE RESEARCH}

\subsection{Introduction and Motivation}

Recently, the use of composite materials has increased in various areas of science and technology due to their high specific stiffness, strength and corrosion resistance when compared to metals. This has especially been the case in the aerospace industries, where fiber-reinforced polymers (FRPs) are used in the construction of aircraft structural members [1]. Composite materials are primarily used within load carrying aircraft structures. Recent examples include the Airbus A350XWB where the composite content has increased to $50-60 \%$ by weight [2, 3]. The Boeing 360, the world's largest helicopter featuring all-composite fuselage, achieves an overall weight reduction of about 25\% over conventional materials. Composites are also employed extensively in the transmission housing, drive shafts, rotor controls, hubs, blades, and landing gear components of the Boeing 360 [4]. Another example of the use of composite materials is the structure of Gripen JAS39, a new Swedish fighter aircraft [5]. Similarly, in the passenger aircraft Boeing 757/767 it has increased gradually from 5\% of the total weight in 1980s to approximately $50 \%$ in Boeing 787. The world's largest passenger aircraft Airbus 380 uses composites of about $25 \%$ of its total weight [6].

In today's aerospace industry, fiber reinforced composite structures require strong joints for aircraft wing and tail assemblies [7]. Joints have an important role in transferring loads between various parts of an aircraft's assembly [8]. These joints must sustain high tensile, compressive, shear, bending and fatigue stresses. The joining of composite parts on a structure often requires the manufacturing of holes so that bolts or rivets can be inserted. Since manufacturing a complex composite structure with a final shape (without machining process) is not feasible, hence the assembly of one mechanical component with another is unavoidable. This assembly problem results in the inevitable application of joints in manufacturing processes $[9,10]$. It is clear that adhesive bonding and mechanical joints are the two widely used joining techniques. With adhesive joints, the load is distributed over a larger area than in the case of mechanical joints, though adhesive joints require special surface preparation and are not easily disassembled for inspection and repair. In addition, the service temperature and the level of the moisture limit the field of application of the adhesive joints. Consequently, mechanical joints are generally preferred and are largely used in aerospace applications [9]. 
Each part of the bolted joint has the tendency to deform individually. Furthermore, bolted joints are always considered as a source of damage initiation and propagation during cyclic loading as they represent the weakest part of an assembly [7, 8]. Hence the efficiency of an assembly largely depends on the joints. Also, poor selection of the drilling process, non-optimal machining parameters and tool geometry results in delamination and matrix degradation due to the overheating of the cutting tool. Therefore, the strength and life of the bolted or riveted joints are highly influence by the quality and accuracy of the hole [11].

To obtain holes to join various components of a structure, different processes of machining can be used such as conventional and non-conventional machining processes. Different conventional techniques used for composite materials are drilling, turning, sawing and grinding by using a suitable cutting tool and process parameters, whereas water-jet, laser, plasma and ultrasonic machining are considered as non-conventional machining processes [12-15]. It is important to mention that drilling holes with these machining processes leads to the destruction of the fiber continuity of the laminates. These discontinuous fibers have a significant impact on the function and final failure of the assembly parts because of the discontinuity of the load transfer in these fibers. To avoid this problem some authors have proposed to replace drilled holes by molded holes [12-15]. Composite parts with molded holes offer better failure strength compared to the composite parts with drilled holes. However, experimental tensile tests conducted by [12-15] have showed that the first damage of the specimen with a molded hole was observed for small tensile load compared to the specimen with a drilled hole. Hence, for joining various components of a complex structure, we often require drilling operation as it is a commonly used technique for producing holes in aircraft industries $[9,10]$. Holes produced are used in order to join various components in a structure with the help of bolts or rivets in accordance to the customer requirements [2]. For instance, a typical wing of an aircraft may have as many as 5000 holes [16]. A bolted or riveted joint transfer load within the structure and its strength and life is dependent on the machined surface. Defect-free machining of holes results in cost reduction and increased fatigue life of the composite structures [11].

Conventional drilling usually applied to isotropic materials is generally less efficient for anisotropic materials like composite material [17]. Nevertheless, with this process of machining the obtained holes are often accompanied by several damages in the composite structure. The occurrence of these damages is a major concern in the machining of the composite by the 
conventional machining process. From the literature review, these damages can be classified into three zones: (1) delamination at the hole entry, (2) degradation of the resin at the wall of the hole $[3,12-14,18]$ and (3) delamination at the exit of the hole in the last plies, which is generally due to the cutting tool's thrust force [13]. Machine induced surface damages includes, delamination, cracks, thermal degradation of the matrix and the abrasive nature of reinforcement fibers causes fast tool wear, leading to the repeated change of tool [19]. These are the main factors for poor surface quality, which considerably decrease productivity and product quality [15]. Even though the CM process is utilized very frequently in today's manufacturing by selecting suitable cutting parameters and tool geometry, but most of the problems mentioned above remain the major cause of producing surface damage [11]. Such damages can cause significant reduction in tensile and compressive strength and it also decreases stiffness [2], consequently reducing the performance of the entire structure [11] which adds to huge economic burden, as $60 \%$ of the rejected components during manufacturing owe to the inferior hole quality $[1,11,18,20]$. Nonoptimal cutting tool designs, rapid tool wear, and machining conditions can be considered for the above mentioned problems. Such anomalies also arise from the abrasive nature of the reinforcing fibers [15]. Hence higher quality of the machine holes translates in the higher quality of the structure joints [19]. Therefore the machining of a hole with a conventional machining process is a challenging task in manufacturing industries as of different mechanical properties of each layup of the composite laminate [19]. In order to produce low cost, efficient and lean aircraft manufacturing, machining processes and conditions must be studied in detail.

Currently, a lot of research work on the conventional machining of composites has been done, however, the machining of composite materials is still evolving and is not fully understood yet, especially the influence of machined surface behaviour on the mechanical properties. All previous research works are mainly concentrated on the effect of the process parameters, hole geometry, thrust force, cutting speed and feed rate on the delamination. Since the machined surface texture is an important parameter in evaluating the fatigue performance of the machined surface, it is also of paramount importance for selecting a suitable machining process for engineering components. Therefore, there is a need to select a cost effective machining process in order to minimize the problems stated above to obtain optimum fatigue life. Hence it is proposed to obtain holes through non-conventional machining like water-jet drilling with the addition of abrasive materials. Abrasive water-jet machining (AWJM) can be used for metallic 
alloys, ceramics and composites for through cutting, milling, turning, cleaning etc. [21]. Waterjet cutting is widely used in the machining of composites as it has good preconditions for cutting composite laminates and also reduces the problems made by CM process [12]. It is well known that a machined surface produced by AWJM is free from abnormalities like thermal or nonthermal stresses along the cutting path [22], matrix degradation, fiber pull-out and delamination [22-24] as a low thrust force is produced during this technique [25]. It also eliminates secondary finishing process [22-24]. Minimal force is exerted on the work piece, no smoke or dust is generated and no workpiece distortion occurs during this process [23]. In conventional machining, energy is released in the form of heat when the material deforms or is damaged and fissured in an irreversible way [12, 26]. These thermal damages can be prevented as there is no significant rise in temperature during AWJM process [23, 24] and the machined surface produced is almost without any heat affected zone [24]. This technique can be effectively used for the quick drilling of holes in composites [3].

Based on the existing literature, it is known that for conventional machining (CM) the effect of the drilling parameters was mainly studied. Only few studies were interested in investigating the effect of the machining quality on the mechanical behaviour of the composites. Most of these works focus only on conventional machining such as axial or orbital drilling. To the best of my knowledge there is no study available in literature that investigated the influence of conventional and non-conventional machining processes on the mechanical behaviour of composite laminates using infrared thermography and correlated the fatigue damage mechanisms of the structure parts to the surface quality of the machined holes drilled with conventional and abrasive water-jet machining processes. In addition, there is no research work available in open literature that correlates the mechanical behaviour of the assembly specimens drilled with abrasive water-jet machining process to the surface integrity. Additionally, at present no criterion was developed for the characterization of the surface of composite laminates after machining, however, currently surface roughness $(R a)$ is used instead, which is successfully using for metallic material.

All of the above discussion needs an extensive research study to explore a machining process which will be suitable for composite laminates used in aerospace field. For this purpose a comprehensive research program was planned to analyze machined induced damage, which is presented in the following section. 


\section{$1.2 \quad$ Research Objectives}

The main objective of this research is to investigate the influence of the surface topography induced by conventional and non-conventional machining processes on the mechanical behaviour of the CFRP laminates of different stacking sequence to minimize the issues discussed in Section 1.1 in order to obtain longer life of the structural parts and assembly during working load.

In order to achieve the aforementioned main objective, the following sub objectives need to be realized:

1- Compute and analyze the material and mechanical properties of CFRP orthotropic composite laminates using analytical analysis combined with experimental testing.

2- Investigate the effect of the surface texture on the mechanical behaviour of the drilled single rectangular composite plates during static and fatigue testing.

3- Assess the suitability of the surface roughness criterion $(R a)$ for the composite materials

4- Correlate the surface integrity to the damage mechanisms using surface roughness measurements

5- Characterize the progressive damage mechanisms in the composite specimens during fatigue testing

6- Develop a suitable new non-destructive damage criterion to quantifying this damage for the machined composites.

7- Assess the effect of the surface quality on the mechanical behaviour of assembled single-lap, single-joint composite specimens during static and fatigue testing.

To achieve the above sub objectives, we have divided the current research into two phases: analytical and experimental.

In the analytical phase, the stress concentration around the machined hole will be studied in order to develop a criterion to understand the effect of the stress concentration on the conventional and AWJ machined surfaces. For this purpose, a thermal concentration factor (TCF) will be developed and will be calculated based on the heat dissipation from composite laminates. This will be done using the infrared camera software called ALTAIR. Then the infrared stress concentration factor (SCF) will be calculated from another software called ALTAIR LI. Finally, TCF and SCF will be correlated with standard stress criteria, which is average and point stress criterion. Along with the stress concentration factor, a thermographic damage criterion (TDC) 
will be developed to quantify the damage mechanisms in the composite specimens. The TDC will relate thermal threshold area with damage accumulation, thereby displaying the influence of the applied machining process on the mechanical behaviour of the composite laminates.

The far field stress in the transverse direction $\left(\sigma_{\mathrm{x}}\right)$ and stress at the circular hole will be analyzed using the lamina theory for composite materials. These stresses will be compared to those obtained from experimental testing and will be used to investigate the influence of machining process on the mechanical behaviour.

Phase II of the current research will present the experimental study performed to investigate mechanical behaviour of the composite laminates drilled with two different machining processes using different processing parameters and laminates with different stacking sequence i.e. angle ply $\left(\left[ \pm 45^{\circ}\right]_{2 S}\right)$ and quasi isotropic $\left([90 /+45 / 0 /-45]_{S}\right)$ lay-ups. Experiments will be performed by using two sets of specimens namely: structural part (single rectangular plate) and single-lap, single-bolted joint composite assembly.

In the experimental phase of the study the following analyses will be conducted:

First, the stress-strain analysis in static test will be performed using an extensometer and image correlation to determine the influence of machining (abrasive water-jet and conventional drill bit) based on the measurement of deformations under tensile load. Next, detailed fatigue experimental examinations will be carried on a single structure part as well as on the composite assembly of different lay-ups. The stiffness degradation and the damage accumulation in the machined composite specimens will be calculated and correlated to the thermal damage obtained from the IR camera. The endurance limit for the composite specimens will be calculated from the variation of the temperature $(\Delta \mathrm{T})$ and the stress $(\sigma)$ obtained from the thermal camera.

For the assessment of the surface quality (i.e., integrity) of the machined composite specimens, the surface microstructure and the surface roughness of the hole will be measured by means of standardized roughness parameters ( $R a, R v, R s k)$. The topography of the surface of the hole will be then compared with those obtained from scanning electron microscopy (SEM) observations.

\subsection{Dissertation Organization}

This study consists of two main sections. The first section is related to the introduction and literature review, whereas the second section presents the analytical and experimental analysis. Chapter wise detail is given below: 
CHAPTER 1 gives the introduction and background information of the research topic. This chapter introduces the problems faced during drilling holes with conventional machining and explains the need for an alternative machining process. The motivation behind present research is also described here along with dissertation organization.

CHAPTER 2 is a literature review dedicated to studying the effect of conventional and nonconventional machining process on the drilled surface. The influence of machined surface roughness on the fatigue behaviour is also summarized from the literature to understand the criterion used previously for composite failure during service life. Furthermore, the effect of various machining parameters is also studied to acquire sufficient knowledge to select suitable machining parameters for drilling of composite laminates for current research.

Experimental details including the material used for tests, specimens details, experimental setup and tests procedures for the static and fatigue tests will be discussed in CHAPTER 3. Details regarding surface microstructure analysis are also given in this chapter. CHAPTER 4 presents an analytical analysis of orthotropic laminate. Local stresses are analyzed using Hook's law. Further, this chapter presents the development of thermal concentration criterion and will validate it with stress concentration, point and average stress criterion. Stresses at and far from the circular hole will be investigated in this chapter to find the influence of machining process on the mechanical behaviour. CHAPTER 5 presents stress vs strain results of the specimens drilled with two machining processes, during static tests. Strain will be analyzed in this chapter, using images obtained from CCD camera. At the end of this chapter, a brief summary of the chapter is presented.

To fully understand the cyclic behaviour of composite laminates, the process of damage growth and the progressive damage accumulation in fatigue tests will be quantified and is presented in the CHAPTER 6. CHAPTER 6 first presents the stiffness reduction E (modulus of elasticity) for each cycle using the stress-strain graphs obtained from the data acquired from the extensometer and then stiffness degredation values will be used to draw damage accumulation trends to evaluate two machining processes. Surface roughness tests results will also be analyzed to find out whether the surface roughness is an appropriate criterion for the composite laminates or not. An infrared camera was also employed in the experimental work to evaluate the variation in the temperature to explore the machined surface behaviour, which is presented in CHAPTER 6. The variation of temperature $(\Delta \mathrm{T})$ versus the stress $(\sigma)$, was obtained from the ALTIAR and 
ALTIAR LI softwares to find out the point of inflexion (change in the slope) which is the endurance limit and is presented in this chapter. An appropriate damage criterion is also developed and is presented in CHAPTER 6 to evaluate a suitable machining process. At the end of CHAPTER 6, a summary of the chapter is presented to identify a suitable drilling process for composite machining.

CHAPTER 7 presents the local and global damage assessments of the assembly specimens to quantify and follow the damage propagation on the machined surface. At the end of the thesis, in CHAPTER 8, concluded remarks and a list of the contributions to the current study will be presented along with a summary of the future work. 


\section{CHAPTER 2. LITERATURE REVIEW}

This chapter presents the literature review, conducted to improve the quality of machined surface by proper selection of a drilling process. It was investigated that the surface quality of the composites drilled with conventional machining is strongly dependent on the machining parameters such as tool geometry, cutting forces (thrust and torque) [13], the spindle rotation and feed rate of the tool [14]. Thus, in the current chapter the effect of machining parameters on the drilled surface and then on the mechanical properties of the composite material is presented. In addition, this literature review is presented to investigate the effect of stress concentration around circular hole. In the next section the effect of machined surface on the fatigue behaviour, is presented. Section six presents the literature review to select a suitable non-conventional machining process for current research to obtain holes in composite laminates. In the following section, the literature review of the non-conventional machining parameters is presented. Section nine presents the effect of machining parameters on surface roughness and then the roughness effect on the mechanical behaviour during fatigue testing is presented. A literature review related to the fatigue behaviour of the composite structure is presented in section ten. The fatigue damage mechanism is presented at the end of the current chapter followed by a brief summary of the current chapter.

\subsection{Conventional Machining of Composite Material}

Drilling of composites largely deteriorates the initial stiffness under applied cyclic loading. This loss of stiffness resulted in catastrophic failure of the composite material machined parts. Such a poor drilled surface results in $60 \%$ of the machined parts rejection [27]. In the current section different drilling parameters from literature will be examined for their effect on the machined surface integrity along with the effect of the cutting tool on the drilling surface. Hence to identify the suitable drilling parameters and cutting tool to minimize the surface damage and also to provide bases for the non-conventional drilling process to produce a machine surface comparable with the conventional machining process in order to investigate the influence of the similar (in respect of the machined surface roughness) machined surface on the mechanical properties during static and fatigue testing. 


\subsubsection{Parametric Study}

It is important to study the effect of drilling parameters such as feed rate, cutting speed etc. to minimize or eliminate machining defects during the drilling process. Numerous researchers found a correlation between the feed rate and cutting speed, and their influence on machined surface quality, which is given below:

According to the research of Zitoune et al. [1] during the drilling of holes in composites, the chip coming out affects the hole entry and its wall. At a low feed rate these chips are continuous, whereas dust-like chips can be obtained with the increased feed rate. Continuous chips generally produce high surface roughness as they cannot travel easily from the flutes, which increases torque requirements and temperature, probably causing drill breakage. A spindle speed of 1050, 2020 and $2750 \mathrm{rpm}$ and a feed rate of $0.05,0.1$ and $0.15 \mathrm{~mm} / \mathrm{rev}$ were used for machining the composite. The effect of feed rate was also investigated by Wern et al. [28] where it was observed that damage generated in drilling composites is related to the feed rate and drill geometry. It was also found that surface roughness was increased with an increase of feed rate from $0.1778 \mathrm{~mm} / \mathrm{rev}$. Tsao et al. [29] performed experiments using feed rates of $0.1,0.2$ and 0.3 $\mathrm{mm} / \mathrm{rev}$ and found that the feed rate shows physical significance and has the largest contribution to the overall performance in drilling CFRP laminate. A similar observation was also made by Kim et al. [30] during the drilling of thermoplastic composite such as graphitepolyetheretherketone (PEEK) composite. Similarly Davim et al. [31] considered feed rate and spindle speed for their research. The feed rate of 0.05, 0.1 and $0.2 \mathrm{~mm} / \mathrm{rev}$ and spindle speed of 3500, 4500 and 5500 rpm were used for drilling. It was found that a higher feed rate and spindle speed produced more damages around the hole [31]. In the same thematic Gaitonde et al. [32]

observed that feed rate is linearly related to the damage while cutting speed behaves non-linearly with damage at the entrance of the hole. However, Campos Rubio et al. [33] have shown that during drilling with high spindle speed $(40,000 \mathrm{rpm})$, the increasing of the feed rate does not cause the increase of the damage area. Furthermore, the effect of feed rate and speed on the thrust force and delamination is also presented and is given below in addition the effect of tool geometry and cutting tool material on the hole geometry is also presented here. 


\subsubsection{Effect of Drilling Parameters on the Thrust Force}

Krishnaraj et al. [34] found that high mechanical strength can be achieved at a spindle speed of $3000 \mathrm{rpm}$ and feed rate of $0.02 \mathrm{~mm} / \mathrm{rev}$ because these drilling parameters generated less thrust force compared to the spindle speed of $4000 \mathrm{rpm}$ and feed rate of $0.02 \mathrm{~mm} / \mathrm{rev}$. In [35] it was investigated that increasing the feed rate from 0.05 to $0.23 \mathrm{~mm} / \mathrm{rev}$, resulted in an increase in the thrust force from 48-120 N. It was also observed that decreasing the feed rate improved the machined surface quality but on the other hand it decreased the productivity and increased the tool wear. Similar results were also obtained by [36]. In [37] it was showed that feed rate, cutting speed and drill geometry were the main factors affecting thrust force. Furthermore, it was found that when the cutting speed was kept low, the drill wear rate and productivity decreased while increasing the cutting speed, tool wear increases which increases thrust force. In the same thematic, Fernandes et al. [38] found that when the feed rate increased from 0.03 to 0.12 $\mathrm{mm} / \mathrm{rev}$, the thrust force increased. Similar results were observed by Zhang et al. [39] using feed rates of $0.005,0.01,0.02$ to $0.05 \mathrm{~mm} / \mathrm{rev}$. Moreover Zhang et al. found that the thrust force was responsible for the delamination at the hole exit. Other analytical and numerical studies have also shown that the thrust force was responsible for the damage induced at the hole exit [10, 40, 41]. Lin and Chen [42] demonstrated that drilling carbon fiber-reinforced composite at high speed ranging from 9550 to $38650 \mathrm{rev} / \mathrm{min}$ increases tool wear which resulted in the increase of the thrust force.

\subsubsection{Effect of cutting Parameters on Delamination}

Delamination at the entry and exit of hole has been analyzed by various researchers. A number of research work illustrated that delamination is influenced by the selection of suitable machining parameters [13, 43, 44], the geometry of the cutting tool tip [33, 45, 46], and the nature of its material [47-49]. Moreover these research works found that the thrust force is the main factor for delamination at the hole exit. The Taguchi technique was used [13, 49, 50] to study the influence of drilling parameters on the delamination. It was found that delamination at the hole entry and exit increases with the cutting speed and the feed rate of the tool. For a higher cutting speed and feed rate, the delamination is larger in composite materials. Feed rates of 0.04, 0.08 and $0.15 \mathrm{~mm} / \mathrm{rev}$ and spindle speed of 1000, 1500 and $2000 \mathrm{rpm}$ were used by [49]. [13, 50] used feed rates of 0.05, 0.1 and $0.2 \mathrm{~mm} / \mathrm{rev}$ and spindle speed of 1910, 2547 and $3138 \mathrm{rpm}$ in 
their research. The same results were also observed by [13] by using feed rate of 0.05, 0.1 and $0.2 \mathrm{~mm} / \mathrm{rev}$ and spindle speed of 1910, 2457 and $3138 \mathrm{rpm}$ [13]. Chen [51] carried out the drilling of composite laminates and concluded that, with the increase of spindle speed from 3700 to 6000 rpm, delamination at exit of the tool increased considerably. Furthermore, it was found that by decreasing the feed rate, delamination decreases at the exit which resulted in improved hole quality. Similarly, Kilickap et al. [52] observed the influence of cutting speed and feed rate on the delamination of the machined hole. It was concluded that the higher feed rate and cutting speed has the highest influence on the delamination damage. In [53], it was observed that cracks and the de-bonding of fibers occurred due to the low cutting speed and feed rate ratio. Furthermore, it was found that higher cutting speeds caused a higher cutting temperature which resulted in the softening of the matrix due to the low coefficient of thermal conduction and low transition temperature of the matrix.

\subsubsection{Effect of the Cutting Tool on the Hole Geometry}

Delamination of the first ply takes place at the initial stage of drilling which resulted in the debonding of that ply. Delamination increases with the rake angle as a higher rake angle increases thrust forces. It was recommended to use a small rake angle to reduce or eliminate the lifting up of the first ply which accordingly reduces the defects at entry of the hole [41].

Davim et al. [49] found that a carbide drill performed better than a helical flute HSS drill, when drilling CFRP as it generated less damage. Abrate et al. [15] found that the carbide drill was suitable for drilling composites because of the abrasive nature of the fibers. Similar results were also obtained by Nalrotra S.K when drilling GFRP and CFRP using a carbide drill and a HSS drill. In [54], it is mentioned that tungsten carbide with 6 and 8\% cobalt micro grain (for ISO grades K10 and K20 respectively) and diamond coating, is a suitable tool material for drilling composites when a feed rate of 0.05 to $0.15 \mathrm{~mm} / \mathrm{rev}$ was used. Whereas Manna A. et al. [55], observed that the machined surface quality decreased with the increase of cutting tool diameter above $6 \mathrm{~mm}$. In addition, it was found that a carbide tool produces a smaller thrust force compared to an HSS drill for the feed rate of 50 and $250 \mathrm{~mm} / \mathrm{min}$ [56]. 


\subsection{Effect of a machined Surface on Stress Concentration}

All above mentioned studies (Section 2.1) showed a high contribution in producing inferior machined surface quality, either by increasing or reducing the feed rate and spindle speed for the conventional machining (CM) procedure. Despite the fact that $\mathrm{CM}$ is thought to be the dominant material removal process however, it is appropriate for the areas where the machine part is not under high repeated loading and the damaged surface is not a great concern.

The defects produced during the CM process have a large influence on the strength of the specimen under fatigue load, despite the selection of appropriate machining parameters [11]. This inferior surface quality produces high stress concentration sites. Several researchers considered stress around the vicinity of hole under tensile load. For the drilled composites many analytical criteria have been developed to find the failure modes of CFRP. All these criteria are based on the stress concentration theory around the circular hole area where the damage is developed. These criteria depend on the material properties, specimen geometry and service loads. For drilled composite laminates, the Point Stress Criterion (PSC) is generally used [57, 58]. This criterion describes the stress riser in the vicinity of the hole as a function of the hole's radius. Tercan M. et al. [57] applied same idea to find the critical crack length at the edge of the hole. Eriksson et al. [11] proposed a Damage Zone Criterion (DZC), which described maximum stress in the vicinity of the hole. Toubal et al. [59] used a carbon epoxy composite with a circular hole under tensile load to find stress concentration and the strain field by employing the interferometry experimental technique.

\subsection{Fatigue Behaviour of Composite Plates}

For drilling holes in composite laminates, various non-conventional machining processes have been developed, for example, water-jet cutting, laser cutting, electro-discharge machining (EDM) and ultrasonic cutting [60]. However, drilling is the oldest, still most significant [61] and frequently used machining process for producing holes, using twist drill, for riveting and fastening structural assemblies of the composite laminates, as explained earlier. Nevertheless, with the conventional machining we induce various damages in the composites such as delamination at the entry and the exit of the hole, the pull-out fibers and the resin degradation [10]. Correspondingly, these damages result in the degradation of the mechanical properties during service life. Several works have been keen on the analysis of delamination at the entry 
and the exit of the hole induced by the interaction between the cutting tool (conventional machining) and the composite material. A number of works show that this damage is influenced by the choice of the machining parameters, the geometry of the cutting tool tip [33, 43, 45], and the nature of its material as well as the process of the manufacturing of the composites parts [47, 48, 62]. In literature, currently only a few studies are interested in identifying the influence of damage, induced by the process of machining on the mechanical behaviour [2, 11, 34, 63, 64].

In the work conducted by [2] it was shown that failure loads of specimens with conventional drilled holes are inferior to those with molded holes. In addition, specimens with drilled holes represent brutal fractures and specimens with molded holes have progressive fractures. In the same thematic, experimental tests conducted by Persson et al. [11] used carbon fiber reinforced epoxy subjected to static and fatigue loading. Specimens were subjected to pin loading and uniaxial compressive loading using Kungliga Tekniska högskolan (KTH) method and the conventional drilling process. It was shown that during the fatigue test, failure stresses of specimens with drilled holes using the axial drilling process with polycrystalline diamonds (PCD) dagger drill, were 29\% less compared to specimens with drilled holes using an orbital machining process such as KTH process. This difference in the failure stress can be related to the difference of the quality of the machined surface (i.e., wall of the holes). More precisely with a dagger drill the fibers pull-out and thermal degradation located on the wall of the holes were more important compared to those located on the wall of the holes when the machining was carried out using the orbital process. Krishnaraj et al. [34] observed the effect of drilling parameters on the strength of the drilled hole in a composite material made of glass fiber reinforced polymer (GFRP). It was noted that specimens drilled at higher feed rates failed at a lesser load than specimens drilled at lower feed rates. This can be explained by the fact that high feed rate and speeds provoke more delamination at the hole, which in turn affects the failure load.

\subsection{Challenges in the drilling of Composites}

The following are the challenges faced in drilling composites with the conventional machining process:

- Damage on the inner surface of the hole. 
- It is difficult to attain dimensional accuracy during the drilling of composites because of the difference in the coefficients of thermal expansion in matrix and fiber. Drilled holes are often found to be smaller than the drill used.

- Poor thermal conductivity of the fiber and the matrix favors heat buildup at the cutting region. Heat generated is affected by the high cutting speed and low feed rate.

- Tool life is usually shorter because of the abrasive nature of the composite. Reinforcement fibers cause severe wear by abrasion of the cutting edges. The wear of the cutting edge in turn increases the thrust force. The thrust force was found to be the most controlling factor of the onset of delamination. Hence attention is given in selection of appropriate cutting tool and parameters [65].

- Misalignment of cutting tool and vibration of specimen should also be avoided.

\subsection{Twist Drill Selection}

Drilling is a widely used technique for machining or enlarging, through or blind holes in materials. This technique comprises of a rotating tool moved into a stationary specimen. The tool rotates along its axis of rotation. The twist drill is the frequently used drilling tool, generally available in diameters from 0.25 to $80 \mathrm{~mm}$ [66]. It is a general purpose tool that can be used on wood, metals, plastic and most other materials. Usually drilling needs the secondary finishing process, however in some cases it may not need the finishing process as drills such as solid carbide produce tighter tolerance holes [67].

A standard twist drill in Figure 2-1 shows point and helix angles. A standard twist drill with a helix and point angles of $30^{\circ}$ and $118^{\circ}$ is suitable for drilling steel. For cutting brass, bronze and composites [68] usually slow-helix drills with a helix and point angles of $20^{\circ}$ and $120^{\circ}$ are used, while for cutting plastic a point angle of $90^{\circ}$, can be used. A point angle of $120^{\circ}$ is preferred for composite drilling as it produces less thrust forces compared to point angle of $100^{\circ}$ and $90^{\circ}$. Softer material like aluminum alloys and copper can be machined with a quick-helix drill with a helix angle of $40^{\circ}$ and a point of $100^{\circ}$.

Solid carbide drills are made entirely of tungsten carbide—shank, body and point. Solid carbide drills offer the following advantages [67]: 
- Are capable of higher productivity than HSS twist drills.

- Are self-centering—no centering drill is required.

- Are coated with titanium nitride (TiN) and other types of coatings for increased tool life.

- Give excellent chip control and chip evacuation in most materials.

- Can be reground and recoated to keep tooling costs low.

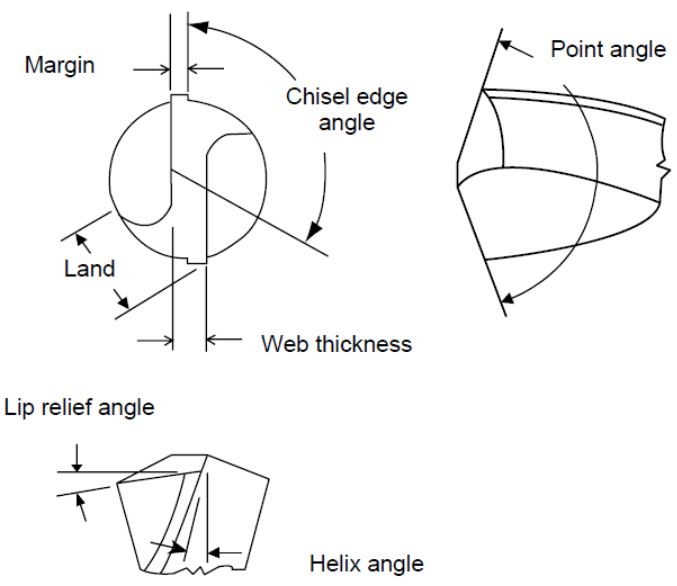

Figure 2-1 Terminology of a standard point twist drill [66].

From the above literature review it is found that the use of a solid carbide tool with a coating of titanium nitride (TiN) is suitable for drilling composite laminates to minimize drilling induced defects.

\subsection{Non-Conventional machining of Composite Laminates}

Conventional drill tips used for efficient metal cutting were designed such that the tip heating the material would provide the plastic flow. However for composites, the drilling process must be slow down to avoid or minimize heating, as composites cannot endure heat. This heat is mostly accumulated due to the pressure exerted by the operator to drill the hole [69]. Furthermore, in the conventional machining process, there are also other damages generated on the machined surface which was discussed in CHAPTER 1. To avoid such problems, it is proposed to obtain holes through a non-conventional machining process. Non-conventional machining is classified according to the form of the energy used for machining such as thermal, electrical and mechanical processes. These processes consist of laser, plasma, electro discharge, abrasive 
water-jet and ultrasonic machining processes. The following study is carried out to select an appropriate non-conventional machining process for drilling composite laminates.

\subsubsection{Selection of a non-conventional machining process}

Laser often generates heat affected zones and thermal distortion when drilling composites and ceramics. Such undesired surface properties after drilling produces rough surface, which needs secondary finishing process that significantly rise the machining cost [70]. Laser is a slow material removal process furthermore; microcracks due to thermal stresses can also initiate during this process. Many materials like copper, brass, glass, ceramics cannot be cut with laser [67]. Moreover it is difficult to cut material more than $50 \mathrm{~mm}$ thickness with laser technique [66].

Electro-discharge machining (EDM) can be used to drill small diameter holes with a good surface finish. This technique can produce more than 1000 holes per second [70]. However this technique is suitable for drilling electrically conductive material [70] and it generates a larger heat affected zone than laser cutting [67]. Ultrasonic drilling can produce a fine surface but the production rate of holes decreases for harder materials [70]. Although plasma cutting is faster than lasers for cutting thick specimens, it is less accurate than a laser [67].

Abrasive water-jet machining (AWJM) can produce holes in hard and soft materials. In comparison with the water-jet technique, AWJM is 10 to 50 times faster. This machining process provides good surface finish. Almost $80 \%$ lower water pressure is required for AWJM than water-jet machining to accelerate water-jet. Abrasive to water ratio is around 3/7 by volume [66]. Abrasives, such as garnet, sand, aluminum, olivine etc. of various sizes can be used for AWJM. This technique generates a finer surface finish (30\% less surface roughness) than WJM [66]. AWJM can be used to machine copper and its alloys, aluminum, steel, tungsten carbide, ceramics, composites, acrylic, concrete, rocks, graphite, silica glass, titanium and alloys etc [71, 72]. In a single pass at a traverse speed of $38 \mathrm{~mm} / \mathrm{min}$, it can cut through $360 \mathrm{~mm}$ thick slabs of concrete and $76 \mathrm{~mm}$ thick steel plates at surface roughness $R a$ from 3.8 to $6.4 \mu \mathrm{m}$ [66]. The

machined surface produced with AWJM is almost without any heat affected zone [73]. This machining process is environmentally friendly as synthetic oils or other chemicals are not required for lubrication and cooling purpose [74]. 


\subsubsection{Parametric Study}

In this technique, a number of parameters play a vital role in the material removal such as abrasive grit size, water-jet pressure and the minimum angle between the axis of the nozzle and the plane of the surface. The influence of these parameters on the surface of the specimen depends on the cutting depth [17]. It is important to point out that the grit size and standoff distance are the most significant parameters affecting the surface finish $[17,75]$ when cutting thin specimens, whereas for thick specimens, grit size and water-jet pressure are the most influential parameters [17] in the abrasive water-jet machining process.

In view of the state of the art research and development that has taken place in the field of AWJM process, a brief literature review is presented here to assess the suitability of this technique according to the various processing parameters to obtain a machine surface integrity comparable to the surface generated using conventional machining process.

\section{Standoff distance}

In [66] it was described that standoff distance (SOD) plays an important role in material removal. Normally 0.5 to $5 \mathrm{~mm}$ can be used as standoff distance. Higher accuracy and the smallest kerf width can be achieved with a smaller standoff distance $(0.5 \mathrm{~mm})$ while a higher material removal rate can be obtained with SOD of $5 \mathrm{~mm}$. However, if the SOD is above $5 \mathrm{~mm}$ till $50-80 \mathrm{~mm}$, the jet cutting ability decreases and in this case the water-jet can be used for surface cleaning [66]. Similar result was also found by Fowler [76]. It was found that the material removal rate decreased with the increase of SOD. Thus, for higher productivity and better accuracy, a shorter SOD is required [71]. With the increase of the SOD to $2.75 \mathrm{~mm}$, the kerf taper increases. However further increase in the SOD decreases the kerf taper because of the expansion of the jet which decreases the energy of the exterior of the jet. This decrease in energy is not sufficient to create macro-damage at the edge of hole [17].

\section{Water-jet Pressure}

The material removal rate is exponentially related to water-jet pressure. An increase in water-jet pressure resulted in an increase in velocity imparted to the abrasive particles, consequently allowing more energy for the erosion of material which finally results in increase of material removal rate. Water-jet velocity can also be increased by reducing the orifice diameter together 
with sufficient abrasive flow rate [71]. Horn G.P et al. [25] used a garnet abrasive of eightymesh size $(177 \mu \mathrm{m})$ with water-jet pressure of $290 \mathrm{MPa}$. The polymer matrix composite specimen of length $152 \mathrm{~mm}$, width $25 \mathrm{~mm}$, thickness $2 \mathrm{~mm}$ and $6 \mathrm{~mm}$ hole diameter was tested at a stress ratio of 0.1 and a frequency of $2 \mathrm{~Hz}$. AWJM specimen induced more damages than the specimens machined with the brad point drills. The author commented that "the increased level of damage from this machining method may be due to this particular combination of materials and machining properties and is not a general condemnation of abrasive water-jet machining." Localized residual stresses of AWJM specimens, the same as generated by shot peening, were investigated by Arola et al. [77] . Such residual stresses are helpful in improving fatigue life as they are compressive in nature. Furthermore, less microstructure changes was observed by Arola et al., which resulted in less deformation.

\section{Abrasives}

Abrasive particles are more generally use for material removal and these are available in the size ranging from 5-500 $\mu \mathrm{m}$. The rate of material removal decreases when an abrasive size of less than $100 \mu \mathrm{m}$ was used [76]. Commonly used inexpensive and generally available abrasives are garnet, sand, silicon carbide, aluminum and olivine [74] while diamond and cubic boron nitride abrasives are much more expensive [78].

The impact of various process parameters used for AWJ machining is given in Table 2-1. This

Table 2-1 Significance of AWJ parameters on machining results [79]

\begin{tabular}{lccccccc}
\hline \multirow{2}{*}{\multicolumn{1}{c}{ Machining results }} & \multicolumn{7}{c}{ AWJ parameters } \\
\cline { 2 - 8 } & $\mathrm{P}$ & $\mathrm{d}_{\mathrm{n}}$ & $\mathrm{l}_{\mathrm{m}}$ & $\mathrm{d}_{\mathrm{m}}$ & $\mathrm{m}_{\mathrm{a}}$ & $\mathrm{d}_{\mathrm{a}}$ & mat \\
\hline Volume removal rate & 3 & 3 & 1 & 2 & 3 & 2 & 2 \\
Depth of cut & 3 & 3 & 0 & 2 & 3 & 2 & 2 \\
Width of cut & 1 & 0 & 0 & 3 & 1 & 0 & 0 \\
Surface roughness & 2 & 2 & 0 & 0 & 1 & 3 & 2 \\
Running cost of cutting & 1 & 1 & 1 & 1 & 2 & 0 & 0 \\
\hline
\end{tabular}


P Water-jet pressure, $d_{n}$ Water-jet orifice diameter, $l_{m}$ Mixing tube length, $d_{m}$ Mixing tube diameter, $m_{a}$ Abrasive flow rate, $d_{a}$ Abrasive particle diameter, Mat Material strength property, 3 Most significant, 2 More significant, 1 Less significant, 0 Not significant

table shows that material removal rate depends on various processing parameters such as orifice diameter, abrasive flow rate and water pressure [79].

The abrasive water-jet machining process also has some disadvantages such as low nozzle life, high noise level (more than 80db), high equipment cost and hazards from the rebounding of abrasives during the machining process [71].

\subsection{Selection and Role of Water in AWJM Process}

Water and air are generally feasible to use in abrasive jet machining. Oils and other chemicals are not environmentally friendly to use in this type of machining process while the huge economic cost of boiler installation discourages the use of steam. Despite the fact that air is highly compressible, the use of air is limited because of the moisture condensation when air is compressed above $5 \mathrm{MPa}$. The abrasive air jet (AAJ) system is appropriate to use for micromachining or cleaning applications as this system operates at around $1 \mathrm{MPa}$. The abrasive water-jet technique operates at $400 \mathrm{MPa}$, however some machines can operate at pressure up to 600-700 MPa and are suitable for cutting both soft and hard material at a lager depth [74]. The water has no direct effect on material removal while machining hard material with abrasive water-jet machining process, hence the water impact pressure cannot be considered. Still, water plays an indirect role in AWJM, as explained below [74];

- Water stores and transfers the energy required to accelerate abrasive particles

- Water guides the abrasive particles and focuses impacts within a small spot

- It flushes debris and abrasive particles away from the working zone to expose a fresh material surface

- Reduces heat due to the friction between the particle and work piece

- Water also provides cooling medium since heat is generated during deformation when machining heat sensitive materials hence AWJM is considered a "cold" machining process. 


\subsection{Limitations of AWJM and CM Processes}

The possible reason of the vacuum reduction, created by the Bernoulli effect, is the increase in wear of the abrasive feed pipe internal diameter and pinholes created in it. The reduction in vacuum due to these reasons consequently reduces the abrasive flow rate which finally affects the material removal rate and the depth of the cut. To reduce these effects, proper monitoring is required. For this purpose a vacuum gauge has to be utilized to measure vacuum to replace the feed pipe at a constant value of $27 \pm 1 \mathrm{~mm}$ of mercury at a machine operating pressure of 258.6 MPa (37500 psi) [76].

The abrasive feed rate is mechanically controlled and so for deliberate bulk changes, such as increase in water-jet pressure, it results in an increased abrasive flow rate that can be accommodated and can be easily controlled to $5 \mathrm{~g} \mathrm{~s}^{-1}$. Prolonged operation of the machine resulted in orifice and nozzle wear which in turn change the characteristics of the abrasive waterjet. Hence it is recommended to change the orifice and nozzle after every 50 hours of machine operation or according to the manufacturer's instruction, to minimize or eliminate these effects. This is necessary for efficient material removal because water pressure effects the abrasive velocity. Periodic calibration of the water pressure gauge should be done; the allowable variation in water pressure is $\pm 3.4 \mathrm{MPa}$. To minimize the variation in water pressure and its effect on material removal, a predictive maintenance plan should be made to replace intensifier and water pump seals after each 500 hours of machine operation, or according to the manufacturer's instructions [76].

For conventional machining, CNC position errors should around \pm 5 microns for all three axes $(\mathrm{x}, \mathrm{y}, \mathrm{z})$. The drill bit should not be worn out. To measure the drill bit diameter for checking its accuracy, a digital internal diameter micrometer should be used and should also be calibrated. Collet chuck should be used for precisely holding the drill bit to avoid any errors during drilling holes.

\subsection{Effect of Machining Process on the Surface Roughness}

Every machined or manufactured surface has certain components that combine together to define surface quality, those are external and internal features. External features include surface topography and chemistry at the interface with the atmosphere while sub surface metallurgy and mechanical properties are considered internal features, as illustrated in Figure 2-2. This figure 
also shows the influence of these features on the functional performance [73]. According to this figure, fatigue is related to the quality and condition of the examined surface. Cracks always initiate from a free surface with the cyclic loading, thus fatigue is considered a surface sensitivity process. The fatigue cracks are usually nucleated from the area of higher cyclic plastic deformations and these are the places of plastic strain concentration [80]. Thus to understand the effect of the conventional and non-conventional machining processes on the surface features, a literature review is presented in the following section.

\subsubsection{Effect of CM Process on Surface Roughness}

Manna et al. [55] found that the spindle speed was a more influential parameter affecting surface roughness ( $\mathrm{Ra}$ ) than the feed rate with the contribution of $60.22 \%$ and $32.49 \%$ respectively when a spindle speed of 200, 800, 1400, $2000 \mathrm{rev} / \mathrm{min}$ and a feed rate of $0.02,0.05,0.08,0.11 \mathrm{~mm} / \mathrm{rev}$ was used for drilling e-glass (alumino-borosilicate glass) fiber reinforced polymer composite.

Similarly, Davim et al. found that the cutting speed was the significant parameter that has the highest influence on the surface roughness $(R a)$ while the feed rate has the highest influence on the delamination. A feed rate of 0.05, 0.1 and $0.2 \mathrm{~mm}$ and a speed of 3500, 4500 and $4500 \mathrm{rpm}$ were used for these tests [81]. Similarly Davim et al. also concluded that the feed rate is the cutting parameter that affects surface roughness [82]. Further Davim et al. [83] used polycrystalline diamond cutting tools to examine GFRP to investigate the influence of machining parameters on surface roughness. It was observed that the surface roughness $(R a)$ increased with the increase in feed rate and decreased with the increase in cutting velocity.

Similar research was performed by Latha et al. [84] and it was observed that the feed rate and drill diameter contributed $66.05 \%$ and $22.57 \%$ to the surface roughness of the machines surface whereas the spindle speed has $1.67 \%$ effect on the surface roughness. Research work discussed in [1], described that the surface roughness increased with the increase in feed rate and vice versa for all diameters, however, the effect of the spindle speed on surface roughness was found to be small (Figure 2-3). Similarly Palanikumar K. et al. [85] found that surface roughness increases with the increase of feed rate. In the same thematic, Sheikh-Ahmad JY reported that feed rates below $0.3 \mathrm{~mm} / \mathrm{rev}$ produced a finer surface finish [79].

El-Sonbaty I. [35] investigated that, by increasing the cutting speed, the surface roughness of glass fiber reinforced epoxy composites machined surface showed better results, however, higher 


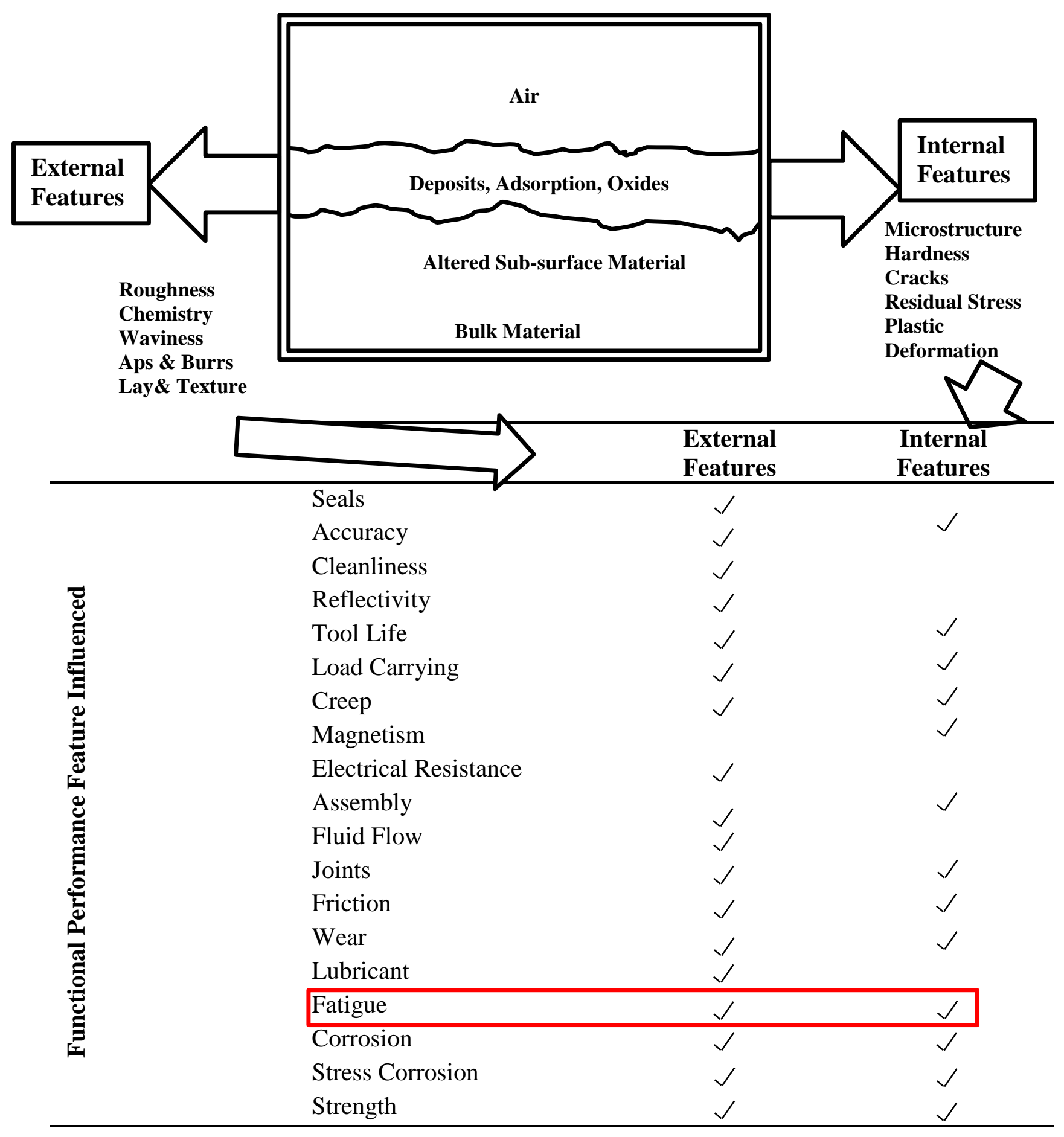

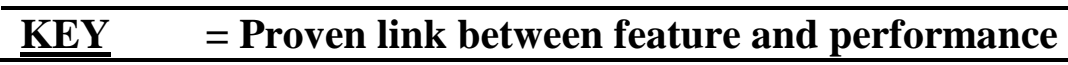

Figure 2-2 Links between surface features and performance [73] 
surface roughness was obtained by a decreasing feed rate. In same thematic Tsao and Hocheng [86] found the feed rate and spindle speed are the parameters that have largest influence on the machined surface roughness. Khashaba U.A. et al. [87] investigated that the fibers were partially sheared from the matrix by increasing the feed rate. However, the complete shearing of fibers can be obtained at a low feed rate and consequently surface finish was improved.

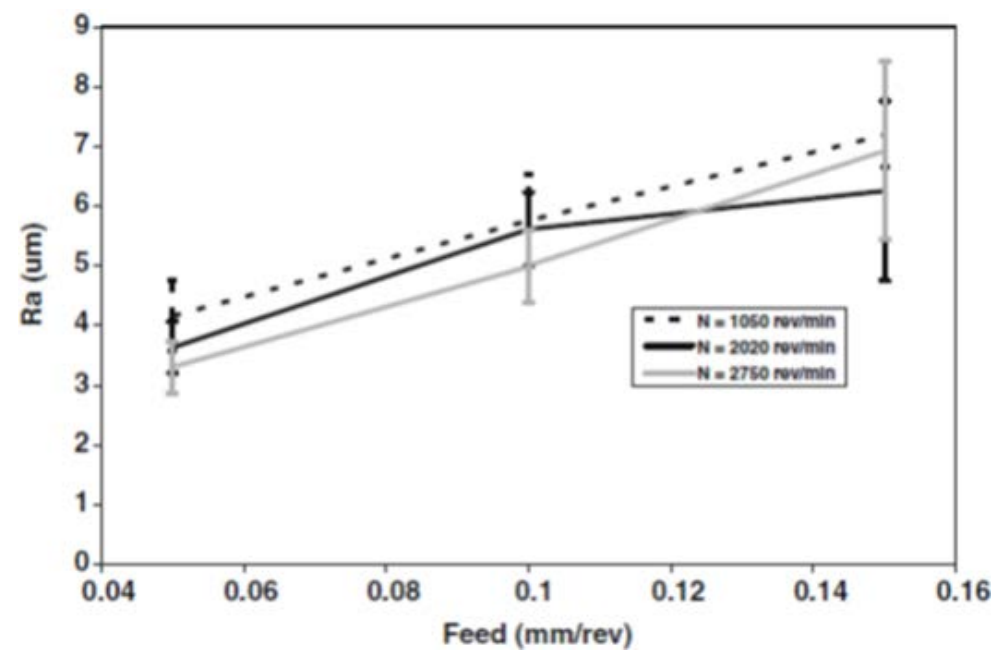

Figure 2-3 Surface roughness $(R a)$ versus feed rate and for various spindle speed for CFRP and drill diameter $6 \mathrm{~mm}$ was used [1].

\subsubsection{Effect of AWJM Process on Surface Roughness}

In case of abrasive water-jet machining, surface roughness largely depends on the abrasive's mass flow rate and particle size. Improved surface roughness can be obtained by using smaller sized abrasive particles [79]. In [66] it was shown that to get roughness of soft material similar to hard ones, generally smaller abrasive particles should be preferred. On one hand, smaller abrasive particles decrease the surface roughness but on the other hand it reduces the depth of the cut and also leaves an undeformed chip along the cutting edge.

In [66], it was shown that the surface roughness of a $5 \mathrm{~mm}$ plate obtained with AWJM ( $R a=4.4$ $\mu \mathrm{m})$ was around $30 \%$ less than that obtained by WJM $(\mathrm{Ra}=6.4 \mu \mathrm{m})$, when an abrasive size of \# 80 mesh (177 microns), an abrasive flow rate of $300 \mathrm{~g} / \mathrm{min}$ and a standoff distance of $5 \mathrm{~mm}$ were used [66]. Surface roughness of $5 \mu \mathrm{m}$ was obtained in [88] when water pressure of $240 \mathrm{MPa}$ was used for the cutting of a $12.5 \mathrm{~mm}$ thick laminate. Further increasing water-jet pressure resulted in a reduction of the surface roughness because higher water pressure extended the size of the 
cutting wear zone as the kinetic energy provided by the higher water pressure increased. The effect of standoff distance on surface roughness was studied in [17]. The water-jet entrance point was mainly affected with the SOD, which also affected the surface roughness of this area. A higher SOD increases the influence of the external drag from the surrounding which expands the water-jet before contacted with the machined surface. This expansion of the water-jet reduces the density of the abrasive particles in the outer portion of the jet. The less dense jet decreases the depth of cut and increases the surface roughness. At a standoff distance of around $2 \mathrm{~mm}$, minimum surface roughness can be obtained.

\subsection{Fatigue Effect on Composite Structure 2.10.1 Introduction}

Composite structures always need joints which should perform efficiently under tension, compression, bending and cyclic loads. Some of the examples of the bolted joints in aircraft structure include; aircraft wing and tail assemblies [7], the wing to fuselage connection and the horizontal/vertical tail connection to the fuselage [8]. Joints are of two types: the lap joint and butt joint. In the composite materials handbook (MIL-HDBK-17) it is given that the lap joint should preferably be used for aircraft structures, since single-lap joint is more representative for the aircraft applications than double-lap joint [89]. Single bolt, single shear joints are preferably used in aircraft structure [90] because of its weight, cost, aerodynamic smoothness and design [91]. Hence in current research the single-bolt, single-joint assembly will be considered for aerospace grade composite laminates.

Bolted joints are always the source of crack initiation and propagation [7] because of the presence of the bolts, which induces stress concentration around the hole [92]. Another source of the crack initiation from the machined surface is the machining technique used for the manufacturing of the circular hole as explained in Section 2.1. In bolted joints, failure usually occurred in two modes, namely, self-loosening and fatigue. Self-loosening generally occurred when transverse or shear load was applied to the joint while fatigue failure was mainly found when the joint was subjected to tensile load [93]. Fatigue failure occurred around the circular hole and the main reasons for this type of failure includes, hole wear, damage in bolt-hole contact surface and drilling induced damage such as delamination in the composite [92]. 
Most of the existing researches are focused on the effect of the stacking sequence, clearance between the hole and the bolt, bolt clamping torque, laminate thickness and the geometric parameters of bolted joints. Most of these researches considered these parameters (mentioned above) to find the fatigue life and strength of the composite material joint [89, 94-101], however none of the studies connected the life and strength of joints with the surface integrity. The following are the few research works in which the decrease in strength and stiffness degradation owing to the damage around the fastener hole was investigated, but none of the research work has focused on minimizing these damages by considering a suitable machining process.

\subsubsection{Fatigue behaviour of the Machined Surface}

Saunders et al. discussed the damage development around a $6.7 \mathrm{~mm}$ thick graphite/epoxy bolted hole assembly under fatigue tensile load. It was found that damage around the fastener hole produces delamination, which may also cause failure of the specimen [102]. Similarly, Starikov et al. [103] found that fatigue damage accumulation strongly affects the relative displacement between the composite plates of the assembly. It was also further investigated by Starikov et al. [92] that at a high applied load on a bolted joint specimen, severe damage was produced around the bolt hole which varied on different bolt holes. Furthermore, Smith and Pascoe [97] observed that the fatigue damage significantly reduced the bearing stiffness of the laminates. Fatigue damage propagation reduced considerably at a higher clamp-up torque of $5.6 \mathrm{~N} \mathrm{~m}$ compared to $2.3 \mathrm{~N} \mathrm{~m}$. McCarthy et al. [101] investigated the effect of the bolt-hole clearance of single-lap, single-bolted graphite/epoxy composite joints on the stiffness and strength. The stiffness of a single-lap joint reduced significantly with the increase of the clearance while the ultimate strain of all tested specimens was increased. Similarly, for larger clearance joints a significant loss in stiffness was observed by Lawlor V. P. et al. [89]. It was found that the reason for this earlier loss of stiffness was the contact loads on the hole which is due to the larger amount of bolted joint damage at the given loading because of the larger bolt-hole clearance. These specimens were further examined with optical microscopy and scanning electron microscopy. Microscopic observation showed that for all specimens significant damage was found at the shear plane of the bolted joints. More significant damage was found for the specimens that had a larger bolt-hole clearance. Moreover, specimens with less clearance were failed by bolt failure, while specimens with larger clearance showed larger displacements before failure. This large displacement owed 
to the larger bolt-hole clearance which created more concentrated loads on the tested specimen and thus inducing more damage in the specimen. In the research conducted by Okutan B. [104], it was shown that ply orientation and geometries, width to diameter and edge distance to diameter ratio of fiber-glass reinforced epoxy single pinned composites joints play an important role on the mechanical behaviour and then on failure of mechanically fastened joints. Aktas A. [105] performed similar tests on glass-epoxy composite plates with single and serial pinned joints and found the failure strength and failure mode. Three different ratios of edge to hole diameters (E/D) were considered, those were 2.3, 4 and 5 while 2, 3.4 and 5 were considered for the width of the specimen to hole diameter ratio (W/D). For the case of W/D=2 (E/D=4) and $\mathrm{E} / \mathrm{D}=2(\mathrm{~W} / \mathrm{D}=4)$ ratios, minimum failure load was obtained, however, the specimens with ratios of E/D and W/D equal to 4, failed at maximum load. Lim T. S. et al. [100] found the effect of the stacking sequence of the bolted joint specimen under fatigue load. Laminates whose major plies were stacked in the axial direction were tested for its suitability for bolted joint structures and it was found that it can be successfully used under cyclic load.

Persson et al. [95] considered various testing factors to find the strength and fatigue life of the composite specimens. Those factors were hole quality, geometry, material properties, laminate configuration and fastener type. For the higher ratio of hole diameter to spacing width, an increase in strength was found during static tests. While during fatigue testing more important ratios were bolt diameter to specimen width and hole diameter to laminate thickness. Moreover, a small deflection of less than $0.05 \mathrm{~mm}$ was noted up to $90 \%$ of the fatigue life and after this deflection was increased more quickly.

\subsection{Fatigue Failure Mechanism}

\subsubsection{Introduction}

Fatigue is considered as the major failure mechanism for composite laminates under cyclic loading. Extensive research is required to investigate the durability and fatigue damage accumulation in the composite material. Presently extensive research has been carried out for metals. For composite materials, fatigue analysis and resulting life assessment becomes difficult since there are different properties of the fiber and matrix. They may considerably affect each other and the interfacial regions between them, under fatigue loading [106]. Fatigue failure depends on many factors including raw material characteristics, magnitude and the orientation of 
applied stresses. Therefore the design analysts must address the implications of various modes of loads [107]. When static load is applied on a material, stress redistribution and local plastic deformation do not contribute to the final failure. The material only fails because of the larger applied load. However, at the yield point, in the case of cyclic load, microcracks and plastic deformation are produced. With a continuous cyclic load, the crack propagated and at particular loading cycle the specimen failed. Fatigue is more complicated than static loading. Fatigue depends on various factors such as the surface quality of the material, intensity and the nature of the applied load on the composite laminate. Thus, fatigue analysis has become an early driver in the product development processes in industries especially in the aerospace industry [107]. Hence to understand the fatigue failure mechanism, its detailed knowledge is indeed needed, which is given below.

\subsubsection{Damage Analysis}

Fatigue damage in composites is quite a difficult phenomena to describe. Literature shows that damage can be defined macroscopically, phenomenologically, physically and microscopically. Macroscopically and phenomenologically it can be described using a secant modulus, residual strength or compliance while physically or microscopically it can be described as the density of cracks, the length of cracks, the delamination area and the number of broken fibers etc. The interaction of various damage types in fatigue loading results in stiffness reduction and degradation in composite properties. So during the service life of composite under fatigue loading it is not feasible to describe the damage evolution via a single damage type. Conversely, a physical description of the damage evolution in a cumulative damage model is difficult to explain. Hence, at present macroscopic and phenomenological fatigue damage are more feasible to use [108]. Damage evolution in a composite is usually described as a reduction in stiffness under fatigue loading. Stiffness decreases continuously with the increase of damage and can be regularly measured without any detrimental effect on the material. Stiffness changes can also correlate with any microscopic damage such as the counting of broken fibers, leaching away the matrix material. Additionally stress redistributions also causes changes in stiffness. Stress redistribution in composite laminates owe to any internal deformation [109]. Furthermore, misaligned fibers, resin-rich regions or voids created during the fabrication process and damage initiated/generated during machining processes are normally considered as crack initiation and 
propagation sites. The continuous damage growth under tension-tension loading in fatigue life was studied and is shown in Figure 2-4. Damage accumulation is shown against loading cycles, where $\mathrm{D}$ is the damage, $\mathrm{N}$ is the loading cycles and $\mathrm{N}_{\mathrm{f}}$ is the number of cycles to failure. Damage

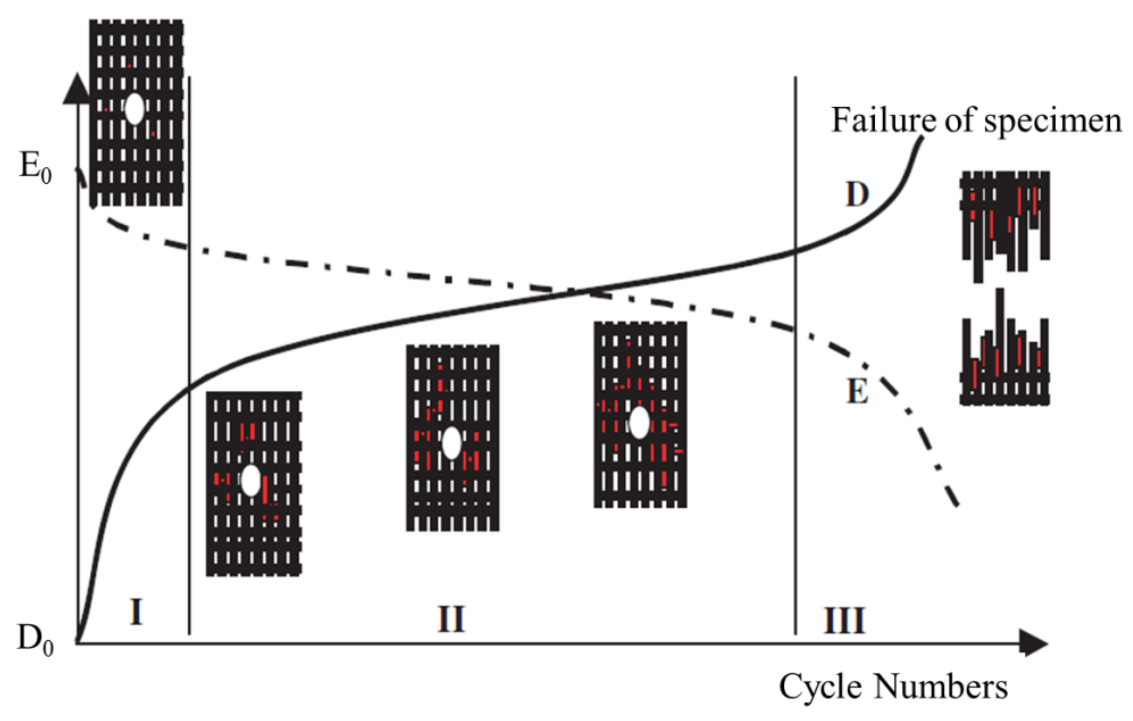

Figure 2-4 The evolution of the modulus of elasticity E vs damage D according to the number of cycle [26]

accumulation is divided in three stages as shown in this figure. Stage I shows the first $20 \%$ of the fatigue life representing multiple crack initiations within the matrix [110]. During this stage debonding among the weak interfaces between matrix and fibers arises along with the breakage of some low strength fibers [106]. The crack initiation continues and grows in multiple mode and number within the matrix and these cracks are the source of the damage accumulation in the stage II. The second stage corresponds to the $70-80 \%$ of the fatigue life. Damage accumulation at this stage causes a reduction in stiffness and matrix-fiber debonding. With the increase of loading cycles the crack grows rapidly between the fiber-matrix interface and it reaches the fiber. Soon, it reaches a stage where the failure stress increases from the loading at a weak point in a fiber, which represents the stage II as shown in Figure 2-5. It causes a reduction in stiffness which results in fiber failure and the carried load is transferred back into the neighboring matrix regions. The inclination of the damage and stiffness curve in this stage is lower than two other stages [110], showing steady damage growth [106]. This damage evolution with the loss of stiffness during the fatigue life of composite laminate is shown in Figure 2-5 as stage II. With a 
further increase of the loading cycles, the fiber becomes too weak to support the applied load and catastrophic failure of fiber occurs, as shown in stage III. This stage has a short fatigue life (about $10-20 \%$ of total fatigue life) but a rapid increase in the damage and a sharp decrease in stiffness is observed [110]. Figure 2-4 also shows the increase in damage as the change in stiffness of the composite laminate. Accumulated damage in this case is given as [26]:

$$
\mathrm{D}=1-\frac{\mathrm{E}}{\mathrm{E}_{0}}
$$

From this graph we can see that $E_{o}$ is the initial stiffness when damage is $D_{o}$ and with the increase in the number of cycles the stiffness reduces and the damage accumulation increases and finally we get damage $\mathrm{D}$ and $\mathrm{E}$ which is the elasticity for the damaged material. Thus the same concept will be used on the current research experimental fatigue data to get the damage accumulation and to investigate whether machining processes influence the damage accumulation of the specimens drilled with the CM and the AWJM process or not. This study will be presented in CHAPTER 6 and CHAPTER 7.

\subsection{Summary}

Based on the existing literature review, it is known that material removal by a conventional machining process significantly deteriorates the fatigue performance of composite materials [2, 111, 112]. From the literature review it was investigated that machined surface integrity during a CM was a major concern and was mainly influenced by the drilling parameters which can also be related with the fatigue life. The feed rate, spindle speed and type of cutting tool were the most influential parameters which affected the machined surface integrity. Moreover, a literature review was conducted to find the optimum machining parameters for CF/Epoxy composite. From literature it can be found that a low feed rate and spindle speed is beneficial for high mechanical strength but it also produces continuous chips which normally produce high surface roughness, consequently raising stress concentration sites. Low feed rate and spindle speed also generated high temperature that was assisted by a low coefficient of thermal conduction of matrix material. This temperature was more than the glass temperature of epoxy which is $150^{\circ} \mathrm{C}$. This high temperature destroyed matrix stability and resulted in a rough surface. The low feed rate also decreases the productivity. Increasing the spindle speed will increase tool wear and 
thrust forces. A high thrust force results in the degradation of the machine surface. So from the literature review it was concluded that the spindle speed of 2020 and $1050 \mathrm{rpm}$ and feed rate of 0.1 and $0.13 \mathrm{~mm} / \mathrm{rev}$ were the suitable parameters normally used for composite machining [1] which will give roughness less than $5 \mu \mathrm{m}$. A tungsten carbide tool of $6 \mathrm{~mm}$ diameter, with a coating of titanium nitride will be used in the current study, which is also suitable for the machining of composite laminates [10, 113]. For the conventional technique, CNC machining will be used as the portable drilling machine produces a vibration and machine errors. Moreover the feed and speed of those machines are fixed.

From the literature review it was found that abrasive water-jet machining (AWJM) is widely used for composites materials. With this process, it is possible to reduce damages that are typical of CM technique [12]. AWJM does not produce heat-affected zones; hence there is no smoke, dust or work piece distortion [23]. Literature shows that a suitable abrasive particle size should be in the range of $100-220 \mu \mathrm{m}$ to obtain a surface finish that will be comparable to the surface obtained with a conventional machining process. The increase in water-jet pressure results in an increase in particle velocity, and hence results in an increase in the material removal rate. Thus for the abrasive water-jet machining (AWJM) process, an abrasive particle size of 120, 220 and 180 microns along with water-jet pressure of 145 and $170 \mathrm{MPa}$ will be considered for angle ply and quasi isotropic lay-up laminates. In current research, structure part and single-bolt single-lap joint assembly will be considered for aerospace grade composite laminates. From literature it was observed that single-bolt single-lap joint assembly is more representative to the aircraft structural joints. For the composite structural assembly, the literature showed that all previous works mainly considered the effect of the bolt and hole clearance, stacking sequence, clamp up force, bolt torque and the geometric tolerance on the strength and fatigue life of the composite material joints. Based on the literature reviewed, there is still room for research to investigate the influence of the machined surface generated from various machining processes on the mechanical behaviour of the single-lap, single-bolted joint composite assembly. To the best of my knowledge, this is the first study that investigates the influence of conventional and nonconventional machining processes on the mechanical behaviour of composite assembly which correlates the damage mechanism of the assembly specimens to the surface quality of the machined holes drilled with conventional and abrasive water-jet machining processes. 


\section{CHAPTER 3. MATERIAL AND METHODS}

In this chapter, all the details regarding the specimen, its preparation and test procedure is presented. The first section describes the composite material used for static and fatigue tests. The second section presents the preparation of the structural part and assembly specimen for the tests. The third section explains the static and fatigue test procedures used for current research while section four provides the details of a global non-destructive thermal detection technique (infrared thermography) which was used as a damage detection technique. The last section of this chapter presents the destructive analysis techniques including surface roughness and scanning electron microscopy used for the microstructure analysis of pre and post fatigue specimens.

\subsection{Material Description}

The main emphasis of this study is to investigate the machining effect on carbon fiber epoxy composite's structural parts and on assembly specimens. For this purpose, two types of symmetric laminates were manufactured for testing namely, angle ply lay-up $\left(\left[ \pm 45^{\circ}\right]_{2 S}\right)$ and quasi isotropic lay-up $\left([90 /+45 / 0 /-45]_{S}\right)$. The composite material used for testing was unidirectional prepregs of $0.26 \mathrm{~mm}$ thickness made of thermoset polymer resin as shown in Figure 3-1.

The carbon fibers and epoxy resin known with their commercial name: HexPly T700-M21GC, were used for the manufacturing of the composite prepregs and were provided by Hexcel composites. T700 carbon fibers can be used in high tensile application with the tensile strength of $4900 \mathrm{MPa}$ and elongation of 2.10\%. Whereas M21GC epoxy resin is high performance tough resin which operates in an environment up to $150^{\circ} \mathrm{C}$ and has a good resistance to moisture. This fiber and resin is currently used in aircraft fuselage structure and in aircraft wings and tail panels. All laminates produced by the autoclave manufacturing technique with 58\% fiber content, $40.4 \%$ epoxy and $1.6 \%$ void content in the resin. The autoclave manufacturing process was used to achieve the optimal mechanical performance of the composites. 


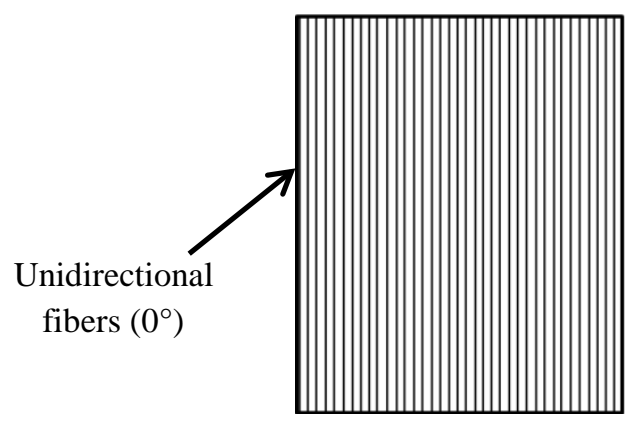

Figure 3-1 Unidirectional fibers used for manufacturing of composite laminates

The curing process for the composite panels was carried out at $180^{\circ} \mathrm{C}$ for 2 hours. During curing, 7 bars pressure was maintained in an autoclave and vacuum pressure was adjusted to 0.7 bars and was maintained during the entire cure cycle. The manufactured composite panels have the final dimensions of $300 \times 300 \times 2.1$ mm (avg.). All panels consist of 8 layers, orientated according to the required stacking sequence. All panels were visually inspected for any manufacturing defects and no visible defects were observed.

The angle ply $\left(\left[ \pm 45^{\circ}\right]_{2 S}\right)$ stacking sequence of the composite panels was chosen for the following reasons. Firstly, since one of the goals was to measure the heat dissipated during the fatigue test, it is important to select a stacking sequence that promotes a non-linear mechanical behaviour in the presence of progressive damages [114]. Secondly, the results of some preliminary fatigue tests conducted on the specimens with unidirectional (UD) stacking sequence did not show any heat dissipation. Thirdly, the mechanical behaviour of these specimens was almost linear and the type of fracture observed was brittle.

\subsection{Specimen Preparation Details}

All specimens (56 No.) were prepared from nine panels. Specimens were made from same mother plate of dimensions $300 \times 300 \times 2.1 \mathrm{~mm}$, to reduce the problem linked to the variability of the mechanical properties of the composite part due to the manufacturing process [115]. All panels were cut using an ultra-thin diamond blade $(0.35 \mathrm{~mm}$ thick $)$-SMART CUT ${ }^{\mathrm{TM}}$ — from UKAM Industrial Superhead Tools, Valencia, California, USA for the preparation of structural parts and assembly specimens. Details of specimen preparation for each type are given below; 


\subsubsection{Structural Part}

Specimens for structural parts were prepared in two sets: the first set of specimens was composed of sixteen composite plates with AWJM holes having a diameter of $6 \mathrm{~mm}$ (5.94 \pm 0.072 ), while the second set consisted of sixteen plates with holes drilled with a conventional drill bit without using a coolant. Wet drilling is recommended when drilled hole depth is twice or more than the drilled diameter. A tungsten carbide drill as shown in (Figure 32) was used for the CM process. It has a helix and point angles of $20^{\circ}$ and $120^{\circ}$ respectively. All test specimens were manufactured according to ASTM D5766/D5766M - 11 [116] standard

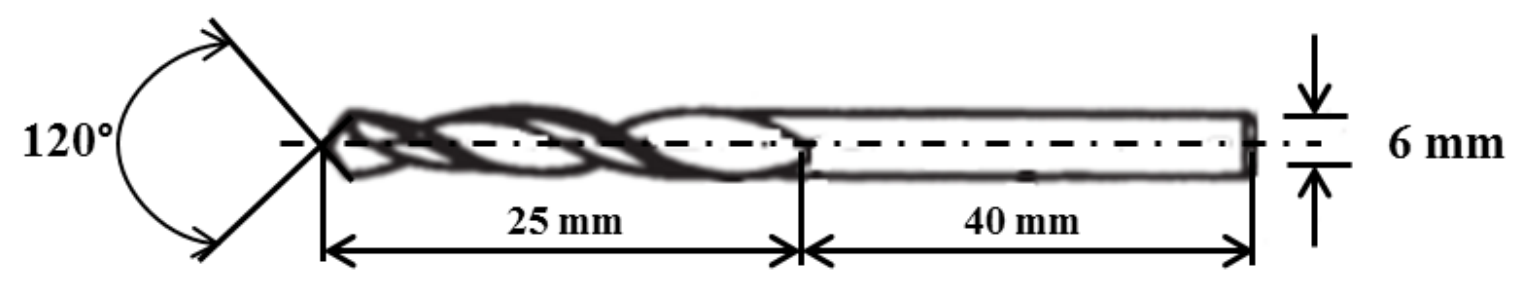

Figure 3-2 Twist drill used for conventional machining

(Standard Test Method for Open-hole Tensile Strength of Polymer Matrix Composite Laminates). According to this standard, the dimensions of the specimens were $270 \mathrm{~mm}$ long, 45 $\mathrm{mm}$ wide and $2.1 \mathrm{~mm}$ thick and the ratio between the hole diameter and the specimen thickness (d/t) was $2.85 \mathrm{~mm}$. The dimensions of the specimen are illustrated in Figure 3-3. In the current case, the end tabs were not equipped since for the open hole specimens, open hole acts as stress riser to force failure in the circular hole region according to ASTM D5766/D5766M - 11 [116].

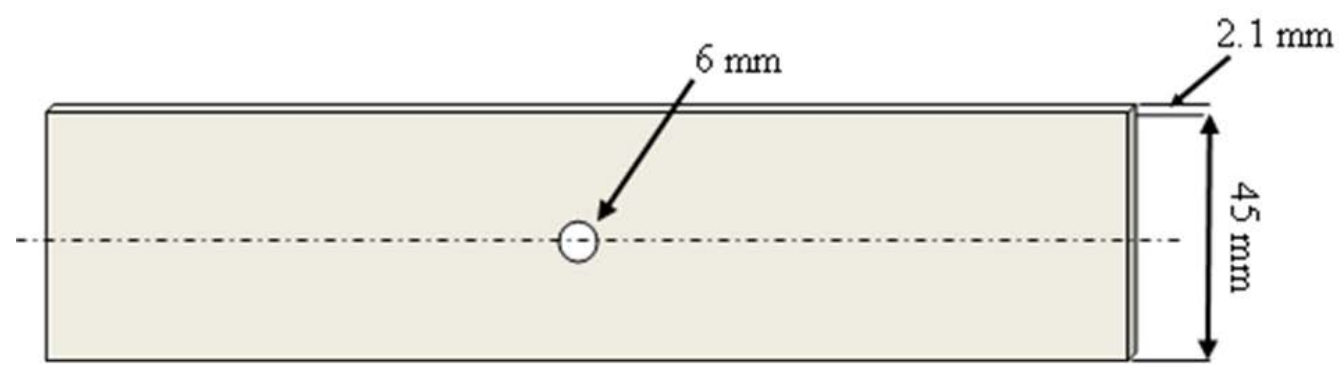

Figure 3-3 Composite plate with centre hole drilled with conventional and abrasive water-jet machining processes, used for static and fatigue testing 
All holes were situated at the center of the plates. For the conventional machining process, a numerically controlled machine was used for drilling holes. During the drilling of the hole in each plate, a fresh area of aluminium backup plate was used in order to avoid bending and interlaminar delamination of the composite laminates, especially the last plies. For the conventional machining process, a carbide drill as shown in Figure 3-2 with two lips was used to drill the holes. The machining parameters were chosen according to the literature review conducted in CHAPTER 2, so as to avoid delamination between plies and to obtain a fine machine surface. For conventional machining, a spindle speed of 2020 and $1050 \mathrm{rev} / \mathrm{min}$, and feed rate of 0.1 and $0.13 \mathrm{~mm} / \mathrm{rev}$ were used, whereas for non-conventional machining, the abrasive water-jet machining (AWJM) process was used for drilling holes. An abrasive size of 120, 220 and 180 microns along with a jet pressure of 145 and $170 \mathrm{MPa}$ was used for the AWJM process to drill holes in laminates with angle ply $\left(\left[ \pm 45^{\circ}\right]_{2 S}\right)$ and quasi isotropic $\left([90 /+45 / 0 /-45]_{S}\right)$ lay-ups. The standoff distance of $4 \mathrm{~mm}$ and water-jet incidence angle of $90^{\circ}$ were kept constant during machining holes. Finally, all machined holes were visually inspected and no delamination or resin degradation was observed.

\subsubsection{Structural Assembly}

For assembly testing, two types of laminates were manufactured, namely, angle ply $\left(\left[ \pm 45^{\circ}\right]_{2 \mathrm{~S}}\right)$ and quasi isotropic ([90/+45/0/-45 $\left.]_{\mathrm{S}}\right)$ lay-ups. In the case of quasi isotropic lay-up laminates, $0^{\circ}$

plies were in the loading direction while $90^{\circ}$ plies were transverse to the loading direction. For each part of assembly specimen, the dimensions were kept according to ASTM D-5961 standard (Standard Test Method for Bearing Response of Polymer Matrix Composite Laminates) [117] as shown in Figure 3-4. ASTM standard, D-5961/D-5961M-96 shows the standard configurations of the composite laminates assembly for the single-lap, single-bolt joint. The circular hole for each part of the assembly specimen was $6 \mathrm{~mm}(5.97 \pm 0.017)$ in diameter and followed the width of the specimen to the diameter of the hole $(\mathrm{w} / \mathrm{d})$ ratio, which was 6 , whereas, edge distance to bolt diameter (e/d) ratio was taken as 3 and the edge distance was taken to be half of the width. To achieve acceptable efficiency of the joint, the overlap to thickness ratio was kept of the order of $17: 1$.

Circular holes were drilled using conventional and non-conventional machining processes. For conventional machining (CM), a computerized numerically controlled (CNC) machine, equipped 
with a carbide twist drill, was used to machine a $6 \mathrm{~mm}$ diameter hole in the center of the specimen with $+0.015 \mathrm{~mm}$ tolerance, in accordance with ISO fitting H7, which is generally used

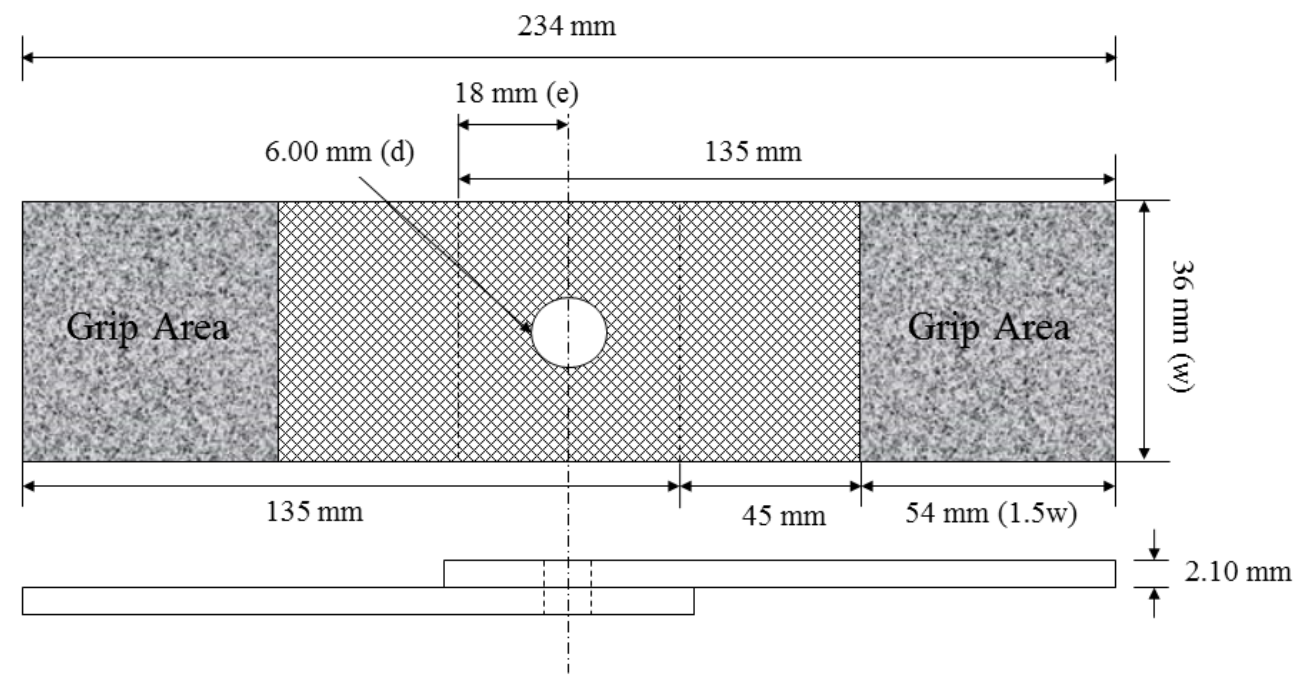

Figure 3-4. Geometry of single-lap, single-bolt and single-shear assembly manufactured from two pieces of CF/Epoxy composite laminates

in the aerospace industry. The CNC machine had a repeatability of $\pm 0.005 \mathrm{~mm}$. For the CM, a spindle speed of 2020 and $1050 \mathrm{rpm}$, and feed rate of 0.1 and $0.13 \mathrm{~mm} / \mathrm{rev}$ were used, whereas for non-conventional machining, the abrasive water-jet machining (AWJM) process was used for machining holes. An abrasive size of 220 and 180 microns along with a jet pressure of 145 and $170 \mathrm{MPa}$ were used for the laminates having $\left[ \pm 45^{\circ}\right]_{2 S}$ and $[90 /+45 / 0 /-45]_{S}$ stacking sequence to drill holes with the repeatability of $\pm 0.07 \mathrm{~mm}$. The standoff distance of $4 \mathrm{~mm}$ and water jet incidence angle of $90^{\circ}$ was kept constant during drilling holes. Machined holes obtained with CM and AWJM processes were inspected with scanning electron microscopy (SEM) and with a surface roughness profilometer for any abnormalities created during drilling processes. These abnormalities will be discussed in Section 6.2.1.

In order to join two composite plates for making an assembly, a proper clamping torque and bolts were selected according to ASTM D-5961 standard to prevent wear due to the sliding, buckling or bending of the two joining parts of the assembly specimen. A clamping torque of 3.4 $\mathrm{N}-\mathrm{m}$ was applied to tight hexagonal socket head steel bolt, using a calibrated micro torque wrench. Grade 12.9 M6 bolts of diameter of $5.92 \pm 0.02 \mathrm{~mm}$, made of alloy steel containing 
chromium and nickel, were used for assembly. The tensile strength of the bolts was $1200 \mathrm{~N} / \mathrm{mm}^{2}$. A standard M6 bolt was used with tolerance according to ISO fitting 6H (5.32/5.21mm). For aerospace structural fastener holes a typical tolerance on fastener-hole clearance is $0 /+0.075 \mathrm{~mm}$ [117]. A steel nut and two washers at the head and nut side of the joint were used. The washers were used to eliminate the through-thickness failure of the assembly specimens [8] and it also prevents bolt and nut rotation.

The end tabs of the length, twice the width of the specimen were made according to ASTM D5961 standard, from the CFRP composite laminates with the same thickness as of the composite plates used for assembly specimens. The end tabs were used to decrease the eccentricity of the loading path, so as to reduce out-of-plane bending moments and to obtain the symmetry of the assembly joint to get uniform line of action of the loading path. To avoid any slippage of the specimen due to the end tab during fatigue testing, ES6228 (resin and hardener) a thin paste bonding material was used. The bonding material was applied to the roughened surface of the end tab and on the assembly parts. The bonded surfaces were pressed firmly in a jig for 24 hours at room temperature to prevent movement during initial curing time. The cured material has a very high strength for tough and durable assemblies. The bonding material used has a tensile lap shear of $24 \mathrm{MPa}$ at $77^{\circ} \mathrm{F}$ and a tensile strength of $65.5 \mathrm{MPa}$.

\subsection{Mechanical Tests}

\subsubsection{Static Loading}

Static tests were performed with an INSTRON 4206 electromechanical testing machine, equipped with a $150 \mathrm{kN}$ load cell. The tests were performed in accordance with ASTM D3039 standard to determine the ultimate tensile strength (UTS) [118]. Static tests were performed on CM and AWJM assembly specimens to obtain ultimate tensile strength and stress-strain behaviour of the specimens. Tests were conducted at a displacement rate of $2 \mathrm{~mm} / \mathrm{min}$. For the local deformation detection technique, an extensometer of gauge length $25 \mathrm{~mm}$ was installed for the measurement of strain values. These deformations were continuously recorded in a small area around the circular hole of the specimens. Furthermore, displacement was also recorded when the specimen was tested under tensile static loads. The specimen's load and deformations were continuously recorded by an appropriate digital data acquisition system to obtain stressstrain curves. We intended to measure the fatigue at different maximum stress levels of ultimate 
tensile strength (UTS). Thus the ultimate tensile strength (UTS) was determined for both laminates to calculate loads applied in each step during fatigue tests. The ultimate tensile strength of CF/Epoxy composite plates with holes can be calculated as:

$$
\sigma=\frac{p_{u l t}}{t \times(w-d)}
$$

where pult is the ultimate force, ' $w$ ' is the specimen width, ' $t$ ' is the thickness and 'd' is the hole diameter [119].

The average value of the ultimate tensile strength was $17.35 \mathrm{kN}$ and $34.78 \mathrm{kN}$, for the case of four $\left[ \pm 45^{\circ}\right]_{2 S}$ and $[90 /+45 / 0 /-45]_{S}$ lay-up laminates, respectively, and the average total elongation was $9 \%$. The ultimate tensile strength (UTS) of four angle ply $\left(\left[ \pm 45^{\circ}\right]_{2 S}\right)$ assembly specimens was $7.18 \mathrm{kN}$ whereas for four quasi isotropic lay-up laminates ([90/+45/0/-45]s) the average value of UTS was $8.32 \mathrm{kN}$. These values of UTS were identical for both processes of machining. The static tensile data don't show any difference in the ultimate failure strength between all the tested specimens.

\subsubsection{Fatigue Loading}

The setup for fatigue testing was comprised of a target (specimen), an MTS 322 mechanical tester and a PC-based image acquisition system (a laptop computer) (Figure 3-5). All fatigue tests were performed using an MTS 322 tester having load cell of $250 \mathrm{kN}$. The specimens were clamped to the MTS tester by wedge shaped grips. ASTM D5766/D5766M-11 standard showed that the length of gripping should be twice of the width of the specimen. Furthermore, the grips were correctly aligned. The specimens were mounted in the grips using a torque of $40 \mathrm{lb}$.in. and was aligned with a spirit level. All tests were performed at room temperature under load control mode using hydraulically operated wedge grips at a speed of $2 \mathrm{~mm} / \mathrm{min}$ and supplied a cyclic load to the composite plate. At the start of fatigue tests, $100 \mathrm{~N}$ of preload was applied, the load was increased linearly at $5 \mathrm{~N} / \mathrm{sec}$, to achieve constant minimum and maximum cyclic alternating load levels, according to the stress ratio $\mathrm{R}=0.1$. A calibrated MTS 244.31 hydraulic linear actuator, MTS 662.22C-01 force transducer with $250 \mathrm{kN}$ capacity and MTS linear variable displacement transducer provided an uniaxial stroke, a recording of force and displacement, respectively. A data acquisition unit was connected with a desktop computer and was used to obtain all the required data. For local deformation measurement an extensometer of gauge length 


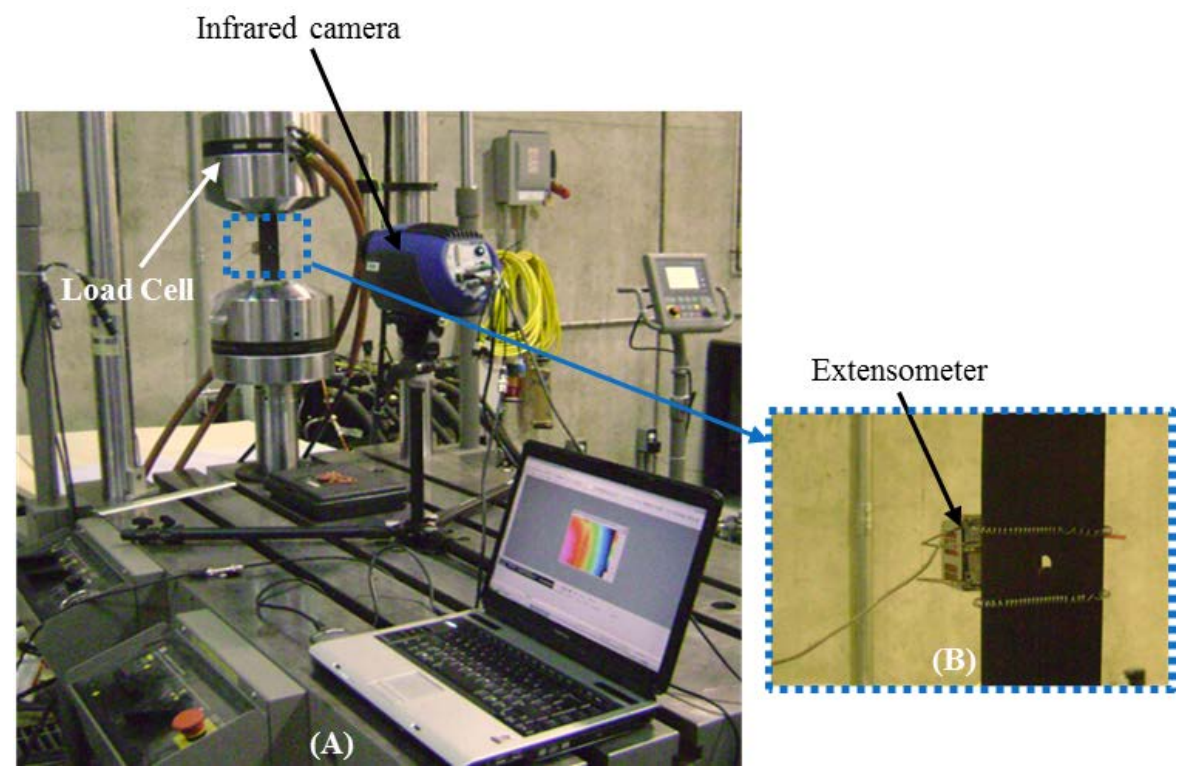

Figure 3-5 (a) Experimental set-ups for fatigue loading for the composite plate, IR camera was positioned at about $100 \mathrm{~cm}$ from the specimen, (b) photograph of extensometer instrumented on the composite material specimen.

$25 \mathrm{~mm}$ was used in the loading axis which was connected with the data-acquisition system of the hydraulic machine and was fixed on the gauge-length section of the specimen (Figure 3-5b). An extensometer was used to monitor the local strain during the cyclic constant loading condition for the calculation of the stiffness degradation of the specimens.

Fatigue tests were performed using a constant amplitude sinusoidal waveform in load control mode, whose minimum and maximum loads are shown in Figure 3-6, with a loading frequency of $10 \mathrm{~Hz}$, at various maximum applied stress levels. To remove any residual stress, static load was initially applied as shown from the first slope. The second slope represents the average value

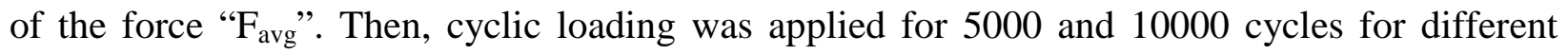
fatigue tests. The cyclic loading was stopped when it reached the maximum number of predetermined cycles set for each step loading test which was 5000 and 10000 cycles. The fatigue test was continuous until the final failure of the structure part and assembly specimens. Uniaxial tension-tension load was applied on all specimens. For [90/+45/0/-45]S lay-up laminates, $0^{\circ}$ plies were parallel to the loading direction while $90^{\circ}$ plies were transverse to the loading direction. For structural parts, the force and deformation were continuously recorded during fatigue tests. For each loading step the data was recorded separately. Step loading fatigue 
tests were started at a low load i.e. $25 \%$ of the ultimate tensile strength, for a predetermined number of cycles. At the completion of the allotted number of cycles, the specimen was cooled to ambient temperature. Once the specimen cooled down to its initial temperature, a load was applied in the next loading stage with a constant increase in the load (30\% of UTS) and this progressive step loading cycles was carried on till final failure of the specimen.

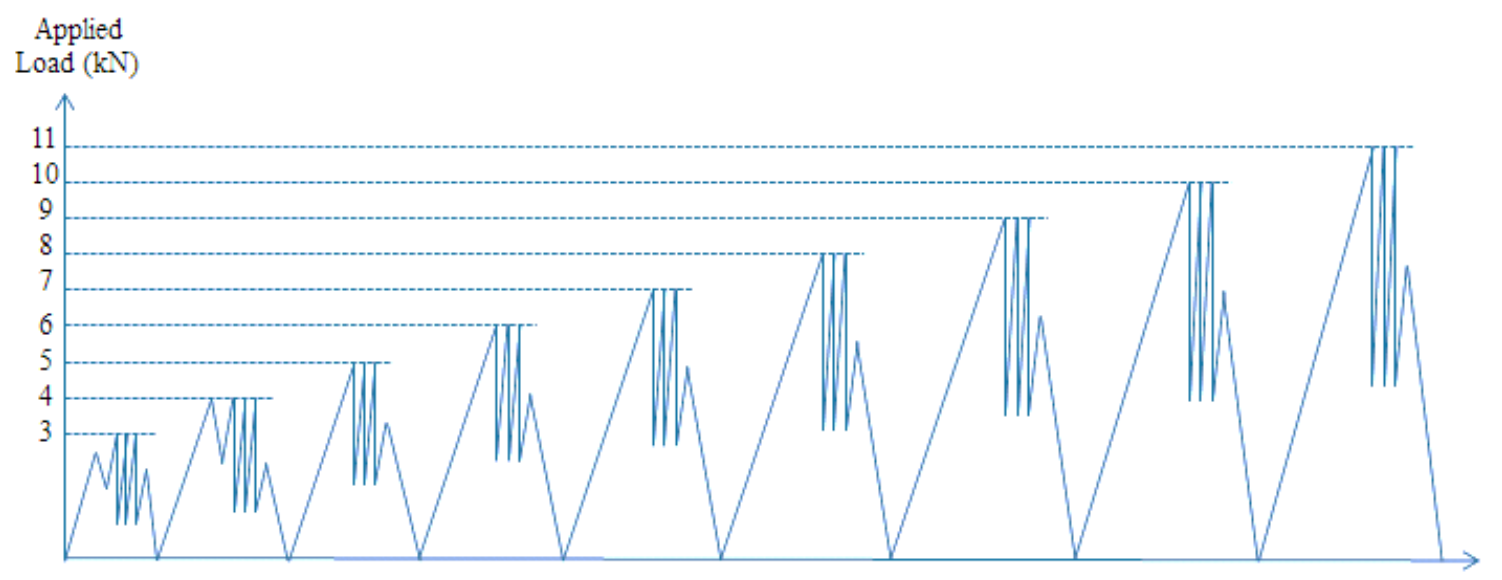

Figure 3-6 Loading pattern of carbon epoxy laminates, increasing loading stages from $3 \mathrm{kN}$ to $11 \mathrm{kN}$ at 5000 cycles for each fatigue loading stage [120]

For each load step, the assembly specimens were fatigue tested for 10000 cycles. The displacement was recorded using the crosshead displacement sensor (LVDT) with a resolution $(\mathrm{dL})$ of 0.003 corresponding to a strain precision $\left(\mathrm{dL} / \mathrm{L}_{0}\right)$ of $0.0013 \%\left(\mathrm{~L}_{0}=234 \mathrm{~mm}\right)$. The edges of the specimens were exactly parallel to each other and both parts of the assembly specimen were perfectly aligned. Furthermore, the circular hole was exactly at the center of the plate, therefore no rotation of the joint was observed.

\subsection{Infrared Thermographic Measurement}

An Infrared thermographic camera (Figure 3-8) was used to monitor heat dissipation during fatigue tests. An IR camera FLIR SC5000 Series was used for this purpose. The camera was fixed on a tripod and placed at a distance of $100 \mathrm{~cm}$ from the specimens gripped in MTS 322 to capture stable images without any vibration and to ensure that the specimens were located in the middle of the image window before taking thermal measurements. The IR camera recorded the fluctuating surface temperature of the specimens by taking a series of images in which 
every pixel represented the average temperature of the matching location. The IR camera was equipped with an integrated built-in auto focusing motorized $27 \mathrm{~mm}$ lens which could also be easily focused using the software. The rectangular specimens made of carbon epoxy composite had an emissivity of $\varepsilon=1$, coefficient of thermal conductivity $\alpha=2.6 \times 10^{-6}{ }^{\circ} \mathrm{C}^{-1}$, and density $\rho$ $=1.44 \mathrm{~g} / \mathrm{cm}^{3}$. An IR camera captures images at a high speed frame rate ( $5 \mathrm{~Hz}$ to $170 \mathrm{~Hz}$ ). For 5000 and 10000 cycles we captured images with a frame rate of one frame per every $4^{\text {th }}$ cycle. An IR camera is a tool used to continuously monitor the specimen surface temperature by taking the infrared signals. The system synchronized these IR signals with the loading signals, in order to be able to produce video images and temperature calculations then carried out. The IR camera has an image resolution of 320 x 240 pixels and provides extremely high temperature sensitivity (a little under $20 \mathrm{mK}$ ). High temperature sensitivity is enabled to detect the heat dissipation resulted from damage growth during tension-tension fatigue loading. This enabled tracking the temperature profile as a function of the number of loading cycles, and to detect damage development during the cyclic loading by comparing the sequence of obtained thermographic images. As we know that the deformation of solid materials is almost always accompanied by releases of heat. When the material becomes deformed or is damaged and fissured, a part of the energy necessary to the starting and propagation of the damage is transformed in an irreversible way into heat [26, 121-128]. Thus this technique makes it possible to measure stress, temperatures etc. remotely and without physical contact with the specimen as it is a non-destructive technique. Hence, in our case, for various loads till 5000 and 10000 cycles, the same sample was used to plot the stress versus temperature until final failure. By analyzing the temperature plots of the external surface during the application of cyclic loading, it was possible to evaluate the damage evolution using thermographic images and to determine the endurance limit [26, 121-127]. A diagram of temperature variation due to thermoelastic heating and heat dissipation during cyclic loading is shown in Figure 3-7 [26].

The temperature measurement images were generated by Altair software (Cedip Infrared Systems, Croissy-Beaubourg, France) and saved for further analysis during fatigue experiments. All tests were performed in laboratory ambient temperature $\left(22^{\circ} \mathrm{C}\right)$ to avoid any temperature variation during infrared thermographic analysis. The test setup for assembly specimens is shown in Figure 3-8. In present research, the experimental work performed with the combination of local and global detection techniques to monitor damage accumulation. 


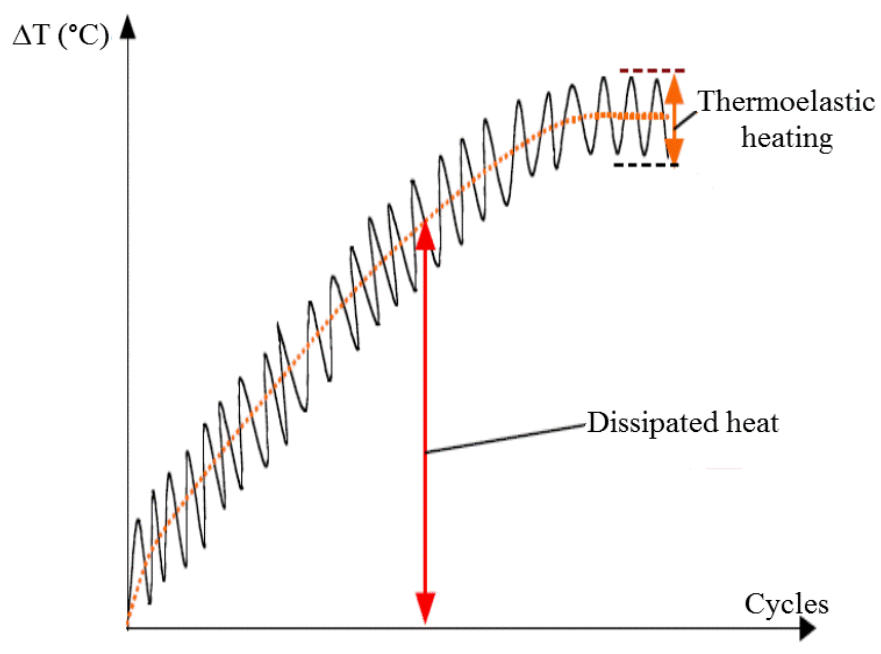

Figure 3-7 Temperature variations during fatigue loading [26]

Local and global detection techniques comprise an extensometer and infrared thermography, respectively, as explained earlier. Accordingly, results of damage accumulation obtained from the extensometer were validated with infrared thermographic observations.

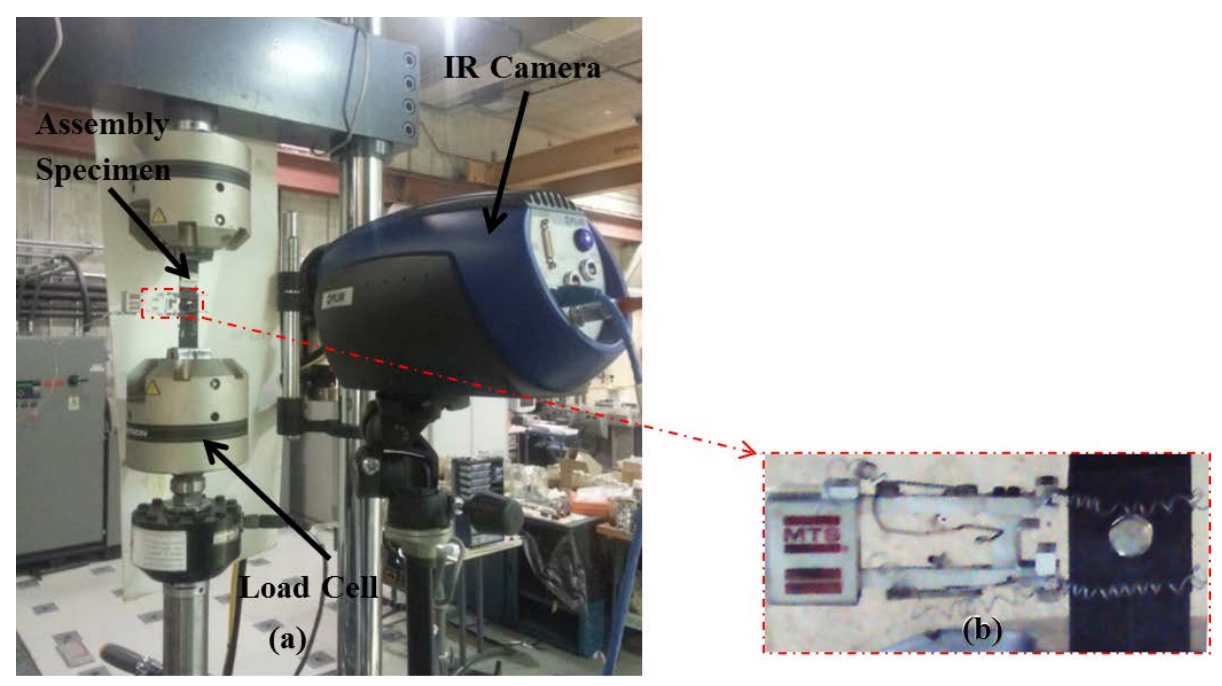

Figure 3-8 Experimental devices for static and fatigue tests of assembly specimens, showing infrared camera and assembly specimen

\subsection{CCD Camera measurement}

Experimental tests were conducted on structure parts using a CCD camera to obtain the digital images of the specimens in the area around circular hole in the directions along $\mathrm{X}$ and $\mathrm{Y}$ planes, with a resolution of $1280 \times 1024$ pixels. This method is a non-contact method based on the 
correlation of the digital images. These images were then analyzed with VIC-2D software.

Digital images obtained from the CCD camera were used to achieve the specimen's deformation and strain concentration around the hole under axial tensile load. Stresses around the hole were calculated from these strain values. Afterwards, these calculated stresses around circular hole were compared to the theoretical stresses calculated from Lekhnitskii’s equation [129].

\subsection{Drilling quality measurement}

In order to investigate the quality of the machined surface and the machined surface texture, a NANOVEA 400 series profilometer and scanning electron microscopy (SEM) were used. For this purpose we cut the specimen's circular hole into two pieces (Figure 3-9). For the profilometer tests, a cut-off and transverse length of 1 and 2 mm respectively along $\mathrm{x}$ and $\mathrm{y}$-axis were considered for surface investigation. The $x$-axis was along the bed of the NANOVEA 400 series profilometer when observed from the front (Figure 3-9). The effects of each drilling process on surface roughness were obtained from the respective surface profile at various locations on the circumference of the hole along its $\mathrm{x}$ and $\mathrm{y}$-axis. As all the specimens were from the same mother plate, there was no material effect on the machining process and on the final surface roughness values. The average surface roughness $(R a)$ was measured according to ISO 4287/1 standards using NANOVEA 3D software to investigate the quality of the machined surface.

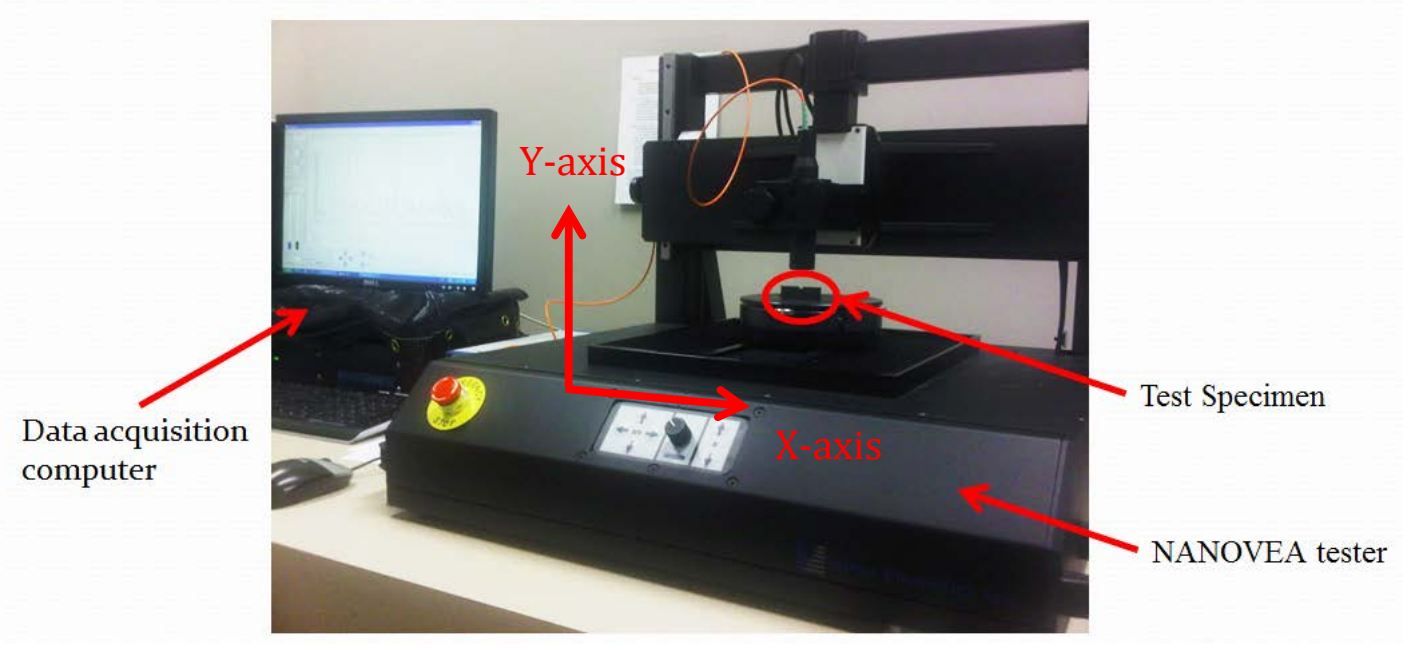

Figure 3-9 Surface Roughness test using NANOVEA 400 series tester 
NANOVEA 400 series profilometer measured surface finish by dragging a skid-type probe with diamond stylus of $10 \mu \mathrm{m}$ diameter, across the specimen. As the probe drifts along a specified area, the roughness profile of the surface was continuously recorded and hence reveals the roughness of the surface. NANOVEA 3D software interprets the obtained roughness profile information. The surface roughness $(R a)$ values obtained from the software, based on centerline average, were mainly used for this study. This data was also transformed by the software in graphical representation.

Pre and post-failure microscopic investigation of the fatigue tested specimens was done using scanning electron microscopy (SEM) JEOL JSM-6380LV to observe the microstructure and to detect the propagation of the damage, the mode of failure and the failure mechanism of the postfatigue CFRP specimens. SEM of pre-failure specimens was also performed to confirm the surface roughness results. 


\section{CHAPTER 4. ANALYTICAL ANALYSIS}

Carbon fiber epoxy composite is currently getting more attention because of its mechanical properties and increasing use in aerospace industries mainly due to its light weight, which considerably reduces the weight of the structure. Carbon fiber epoxy composite is an anisotropic material that has different mechanical properties in a different direction. Therefore, one of the main challenges in composite materials is their machining. Thus, keeping in view these characteristics and the challenge, it is planned to perform an analytical and experimental study of aerospace grade carbon epoxy composite laminates drilled with two types of machining processes. Hence this chapter presents an analytical study of the two types of the laminates. Firstly, analytical stress-strain, elastic properties and Hook's law of the composites are defined, followed by the stress-strain relation for a lamina. In next section, the thermal concentration criterion is developed and verified with the stress concentration factor, point and average stress criterion. Stress at the circular hole and far from the hole is calculated. At the end of the chapter, a brief summary of the chapter is presented.

\subsection{Analytical stress and strains in orthotropic laminate}

In this section, the lamina and laminate material properties and the strain and stress calculations are carried out, considering the specimen is in the linear elastic region of the stress vs strain curve. For this purpose, the local and global coordinate system was considered. The local coordinate system is parallel to the fibers and the global coordinate system is at a fixed reference point of the coordinate system. Figure 4-1 illustrates the local and global coordinate system considered for carbon epoxy composite laminate used in the current study. For the current study, global strains were obtained from the analysis of CCD camera images, while local strain and stress values will be calculated from these global strain values. The global and local coordinates are represented by x, y, z and the 1, 2, 3 axes, respectively. For global coordinates, $\sigma_{\mathrm{xx}}, \sigma_{\mathrm{yy}}, \sigma_{\mathrm{zz}}$ and $\tau_{\mathrm{xy}}, \tau_{\mathrm{xz}}, \tau_{\mathrm{yz}}$ are normal and shear stresses, respectively while the corresponding normal and shear strains are $\varepsilon_{\mathrm{xx}}, \varepsilon_{\mathrm{yy}}, \varepsilon_{\mathrm{zz}}$ and $\gamma_{\mathrm{xy}}, \gamma_{\mathrm{xz}}, \gamma_{\mathrm{yz}}$ respectively. Moreover, for the local coordinate system $\sigma_{11}, \sigma_{22}, \sigma_{33}$ and $\tau_{12}, \tau_{13}, \tau_{23}$ are the normal and shear stresses respectively whereas $\varepsilon_{11}, \varepsilon_{22}$, $\varepsilon_{33}$ and $\gamma_{12}, \gamma_{13}, \gamma_{23}$ are the corresponding normal and shear strains respectively. 


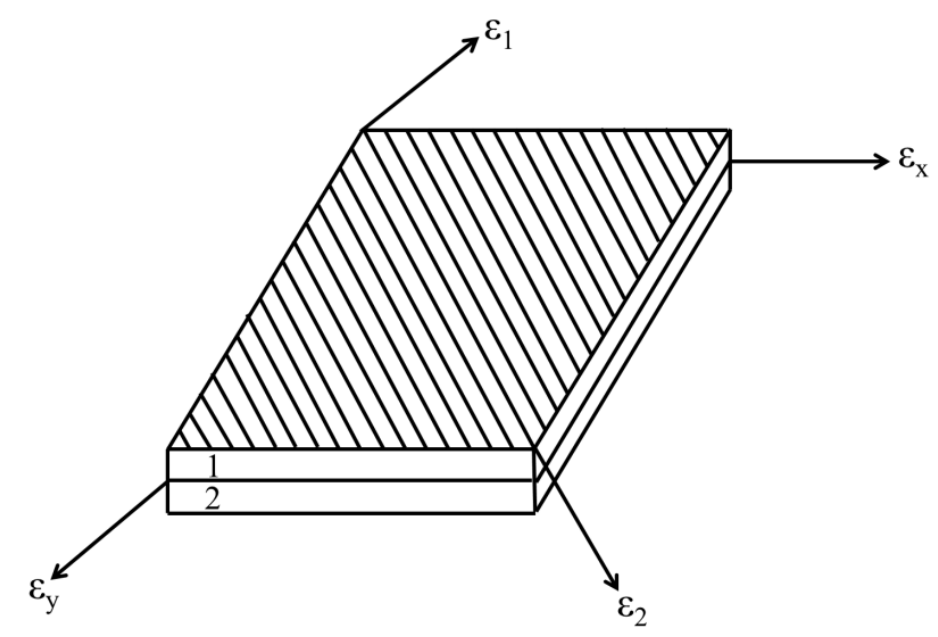

Figure 4-1 x and y shows global while 1 and 2 represent the local coordinate system.

\subsubsection{Calculations for Elastic properties of Composite Laminate}

A unidirectional (UD) composite with uniform fiber properties and diameter is selected. The fibers are continuous and parallel throughout the rectangular specimen. It is considered that there is no slippage between fibers and the matrix and there is perfect bonding between them. Moreover, strain experienced by the fiber and matrix are equal in each ply when the load applied is parallel to the fiber direction. Furthermore, the global strain find from experiments can be assumed as the same for all plies as shown in following equation, when strains act as a parallel springs.

$$
\left.\left.\left.\varepsilon_{\mathrm{x}}\right)_{1}=\varepsilon_{\mathrm{x}}\right)_{2}=\varepsilon_{\mathrm{x}}\right) \text { global from tests }
$$

while stresses of fiber and matrix are different as both have a different elastic modulus, it can be written as;

$$
\begin{gathered}
\sigma_{f}=E_{f} \varepsilon_{L} \\
\sigma_{m}=E_{m} \varepsilon_{L}
\end{gathered}
$$

For the examined specimen, the load $F_{c}$ is applied to the composite laminate, which is equally shared between the fibers $\left(F_{f}\right)$ and matrix $\left(F_{m}\right)$ to maintain equilibrium between the total applied force on the composite in the fiber direction and the sum of forces in the fiber and matrix. So forces inside fiber and matrix can be written as [130]; 


$$
F=A_{f} \sigma_{f}+A_{m} \sigma_{m}
$$

The cross sectional area of fiber and matrix can be written as;

$$
F=A V_{f} \sigma_{f}+A V_{m} \sigma_{m}
$$

Where $V_{f}$ and $V_{m}$ are fiber and matrix volume fractions respectively, now stresses in the above equation can be written in the form of strain as from Equation 4 [130];

$$
F=A V_{f} E_{f} \varepsilon_{L}+A V_{m} E_{m} \varepsilon_{L}
$$

or it can be written as;

$$
\sigma_{L}=\left(V_{f} E_{f}+V_{m} E_{m}\right) \varepsilon_{L}
$$

The above equation can be defined in terms of the elastic modulus of composite which is along the fiber direction, thus the elastic modulus of composite can be written as;

$$
E_{C}=E_{L}=E_{f} V_{f}+E_{m} V_{m}
$$

The longitudinal elastic modulus of the lamina parallel to the fiber direction is $\mathrm{E}_{\mathrm{L}}$, which can mathematically be expressed as [130];

$$
E_{L}=E_{f} V_{f}+E_{m} V_{m}
$$

In the case of the load applied in a transverse direction, the elastic modulus can be found from the following equation [130];

$$
E_{T}=E_{f} E_{m} /\left(E_{f} V_{m}+E_{m} V_{f}\right)
$$

Where $E_{\mathrm{f}}$ and $\mathrm{E}_{\mathrm{m}}$ are the elastic modulus of the fiber and the matrix, respectively, whereas all the values of this equation are expressed in gigapascal (GPa).

Two Poisson ratios are considered, namely, $v_{12}$ and $v_{21}$ which are the major and minor Poisson ratios. The major Poisson ratio is related to longitudinal to the transverse strain while the minor is related to the transverse to longitudinal strain. For major and minor Poisson ratios, the strain is applied in longitudinal and transverse directions, respectively. Hence the Poisson's ratio can be calculated from following equation for a lamina, when the strain is in longitudinal direction [130];

$$
\begin{gathered}
v_{L T}=v_{f} V_{f}+v_{m} V_{m} \\
v_{L T}=\left(E_{T} / E_{L}\right) v_{L T}
\end{gathered}
$$


The fiber and matrix shear modulus in gigapascal can be calculated from;

$$
\begin{aligned}
& G_{f}=E_{f} /\left(2\left(1+v_{f}\right)\right) \\
& G_{m}=E_{m} /\left(2\left(1+v_{m}\right)\right)
\end{aligned}
$$

and the transverse shear modulus of the lamina is;

$$
G_{L T}=G_{f} G_{m} /\left(G_{f} V_{m}+G_{m} V_{f}\right)
$$

\subsubsection{Hook's law for orthotropic composite laminate}

The elastic behaviour of an anisotropic composite laminate is described generally by 21 independent elastic constants and is generally different along each axis. These 21 constants are reduced from 81 elastic constants because of the 3 symmetry relationships as many composite materials have a structure with one or more symmetries. In the present case, for a complete description of the elastic behaviour of composite laminate, the last nine independent constants are required. Moreover, the composite laminates are loaded in the plane of the laminate. Such a loading is called a plane-stress condition in which all out-of-plane stress components are zero. For plane stress condition, the stress is described by nine components of stress tensor $\sigma_{\mathrm{ij}}$ as shown in Figure 4-2, in this case, all terms related to $\mathrm{x}_{3}$ are considered to be zero i.e. $\sigma_{3}=0$.

Similar to stress, there are also nine strain components i.e. $\varepsilon_{\mathrm{ij}}$. All nine stress and strain components are necessary for the complete description of the elastic behaviour of the orthotropic material. The relationship for the two dimensional stress-strain behaviour is given below, which is generally known as Hook's law [131];

$$
\sigma_{i}=Q_{i j} \varepsilon_{j}
$$

where $Q_{i j}$ is the stiffness matrix, representing the elastic characteristics of the material, where $i, j$ $=1,2,3,4,5,6, \sigma_{\mathrm{i}}$ is the engineering stress component, and $\varepsilon_{\mathrm{j}}$ is the engineering strain 
component. $\sigma_{\mathrm{i}}$ and $\varepsilon_{\mathrm{j}}$ are the vector components. So the stress strain relation is modified and is given for two dimensions as below [131];

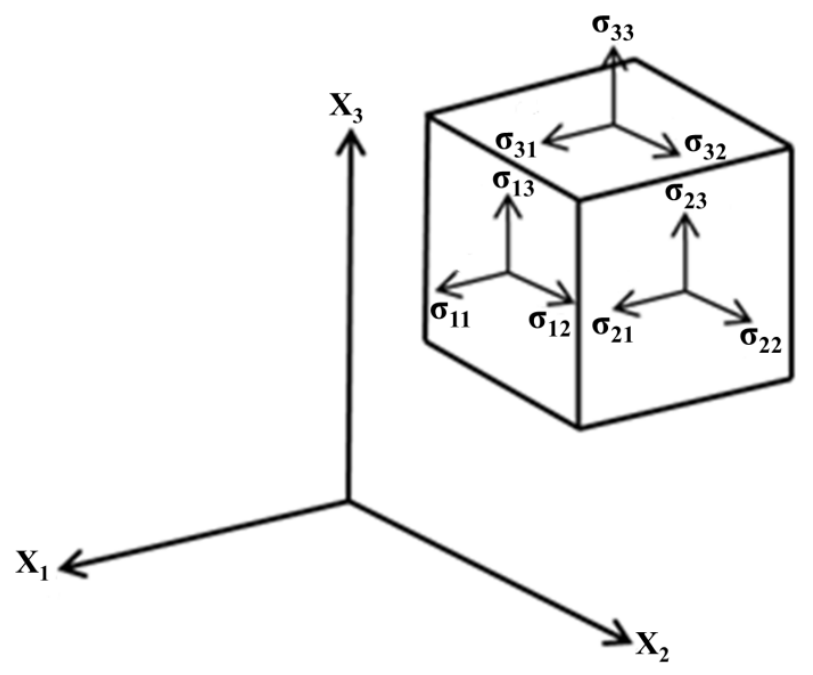

Figure 4-2 Stress components in global x, y, z coordinate system

$$
\left[\begin{array}{l}
\sigma_{1} \\
\sigma_{2} \\
\tau_{12}
\end{array}\right]=\left[\begin{array}{lll}
Q_{11} & Q_{12} & 0 \\
Q_{12} & Q_{22} & 0 \\
0 & 0 & Q_{66}
\end{array}\right]\left[\begin{array}{l}
\varepsilon_{1} \\
\varepsilon_{2} \\
\gamma_{12}
\end{array}\right]
$$

The stress-strain relation given in Equation (13) can be expressed with respect to strain, which is the inverse of Equation (13) as [132];

$$
\varepsilon_{i}=S_{i j} \sigma_{j}
$$

where $S_{i j}$ is called the compliance matrix or compliance tensor. It is found that the compliance and elasticity tensors have same independent components along with five none zero constants, those are, $E_{11}, E_{22}, v_{12}, v_{23}, G_{12}$. So the stress-strain relation for two dimensional orthotropic materials, in terms of a compliance matrix can be written as [132];

$$
\left[\begin{array}{l}
\varepsilon_{1} \\
\varepsilon_{2} \\
\gamma_{12}
\end{array}\right]=\left[\begin{array}{ccc}
S_{11} & S_{12} & 0 \\
S_{12} & S_{22} & 0 \\
0 & 0 & S_{66}
\end{array}\right]\left[\begin{array}{l}
\sigma_{1} \\
\sigma_{2} \\
\tau_{12}
\end{array}\right]
$$

It may be noted that Equation 14 and Equation 16 are inversions of each other. So the following relationships between the elements of stiffness and the compliance matrix may be obtained by the matrix inversion [132]; 


$$
\begin{array}{ll}
\mathrm{Q}_{11}=S_{22} /\left(S_{11} S_{22}-S^{2}{ }_{12}\right) & \mathrm{Q}_{22}=S_{11} /\left(S_{11} S_{22}-S^{2}{ }_{12}\right) \\
\mathrm{Q}_{12}=-S_{12} /\left(S_{11} S_{22}-S^{2}{ }_{12}\right) & \mathrm{Q}_{66}=1 / S_{66}
\end{array}
$$

To solve the components of the stiffness matrix in terms of engineering constants, we obtained [131];

$$
\left.\begin{array}{ll}
\mathrm{Q}_{11}={ }^{E_{L}} /\left(1-v_{L T} v_{T L}\right) & \mathrm{Q}_{22}={ }^{E_{T}} /\left(1-v_{L T} v_{T L}\right) \\
\mathrm{Q}_{22}={ }^{v_{L T}} E_{T} /\left(1-v_{L T} v_{T L}\right) & \mathrm{Q}_{66}=G_{L T}
\end{array}\right\}
$$

Similarly, elements of the compliance matrix can also be derived in terms of engineering constants, these are;

$$
\left.\begin{array}{ll}
\mathrm{S}_{11}=1 / E_{L} & \mathrm{~S}_{22}=1 / E_{T} \\
\mathrm{~S}_{12}=-v_{L T} / E_{L}=-v_{T L} / E_{T} & \mathrm{~S}_{66}=1 / G_{L T}
\end{array}\right\}
$$

\subsubsection{Stress-strain relations for orthotropic lamina}

The eight plies (Figure 4-3) orthotropic laminate with its principle material axes (global coordinate) oriented at an angle $\theta$ which is \pm 45 with the reference coordinate axes (local coordinate) as shown in Figure 4-5. The angle between loading and fiber direction in each lamina is \pm 45 .

Strains values obtained from the experiment can easily be transformed from one set of axes to another, i.e. from global to local coordinate system namely, $x-y$ to $L-T$ axes as shown in Equation (19). The x-axis of strain in local coordinates in Equation (19) is along fiber direction, as shown in Figure 4-4.

$$
\left[\varepsilon_{\text {local }}\right]=[T]\left[\varepsilon_{\text {global }}\right]
$$

The above equation can be written for longitudinal and transverse coordinates as [130];

$$
\left\{\begin{array}{l}
\varepsilon_{L} \\
\varepsilon_{T} \\
\gamma_{L T}
\end{array}\right\}=[T]\left\{\begin{array}{l}
\varepsilon_{x} \\
\varepsilon_{y} \\
\gamma_{x y}
\end{array}\right\}
$$




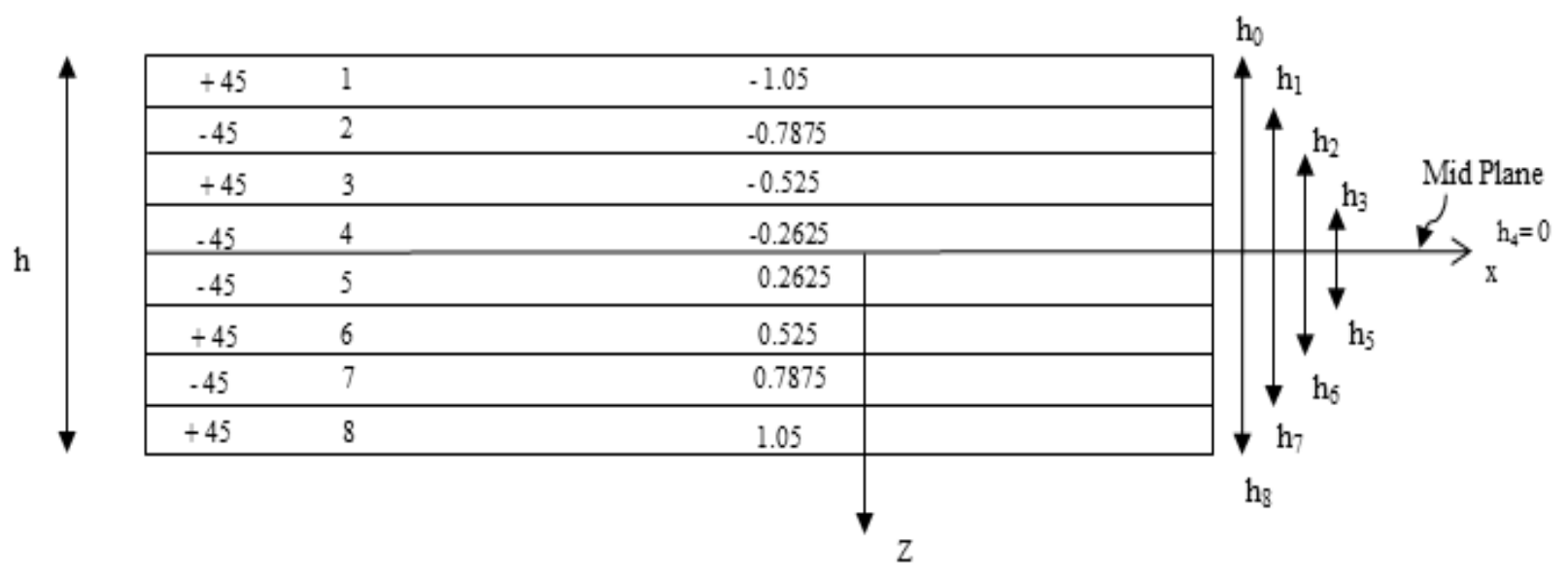

Figure 4-3 Geometry of multilayer composite laminate

For each lamina, Equation 20 can be calculated separately. The transformation matrix $[\mathrm{T}]$ is given by [130];

$$
\begin{gathered}
{[T]=\left[\begin{array}{lll}
Q_{11} & Q_{12} & Q_{16} \\
Q_{12} & Q_{22} & Q_{26} \\
Q_{16} & Q_{26} & Q_{66}
\end{array}\right]} \\
{[T]=\left[\begin{array}{ccc}
\cos ^{2} \theta & \sin ^{2} \theta & \sin \theta \cos \theta \\
\sin ^{2} \theta & \cos ^{2} \theta & -\sin \theta \cos \theta \\
-2 \sin \theta \cos \theta & \sin \theta \cos \theta & \cos ^{2} \theta-\sin ^{2} \theta
\end{array}\right]}
\end{gathered}
$$

It should be noted that the angle $\theta$ is taken as positive when the angle of the L-T axes measured from $x-y$ axes is in a counter clockwise direction as shown in Figure 4-4. Second is that for the transformation of strain, engineering strain is used, instead of tensor strain (then use $\gamma_{x y}$ as $1 / 2 \gamma_{x y}$ ). Thus the simplified Equation (22) is written as [130];

$$
[\mathrm{T}]=\left[\begin{array}{ccc}
\mathrm{m}^{2} & \mathrm{n}^{2} & \mathrm{mn} \\
\mathrm{n}^{2} & \mathrm{~m}^{2} & -\mathrm{mn} \\
-2 \mathrm{mn} & 2 \mathrm{mn} & \mathrm{m}^{2}-\mathrm{n}^{2}
\end{array}\right]
$$

Where $\mathrm{m}=\cos \theta$ and $\mathrm{n}=\sin \theta$

So in linear elastic region from the strain in local coordinate calculated from Equation (20) can now be used to calculate stress in local coordinate using the plane stress formulas as;

$$
\left.\begin{array}{l}
\sigma_{\mathrm{L}}=\frac{\mathrm{E}_{\mathrm{L}}}{1-v_{\mathrm{LT}} v_{\mathrm{TL}}}\left(\varepsilon_{\mathrm{L}}+\varepsilon_{\mathrm{T}} v_{\mathrm{TL}}\right) \\
\sigma_{\mathrm{T}}=\frac{\mathrm{E}_{\mathrm{T}}}{1-v_{\mathrm{LT}} v_{\mathrm{TL}}}\left(\varepsilon_{\mathrm{T}}+\varepsilon_{\mathrm{L}}\right)
\end{array}\right\}
$$




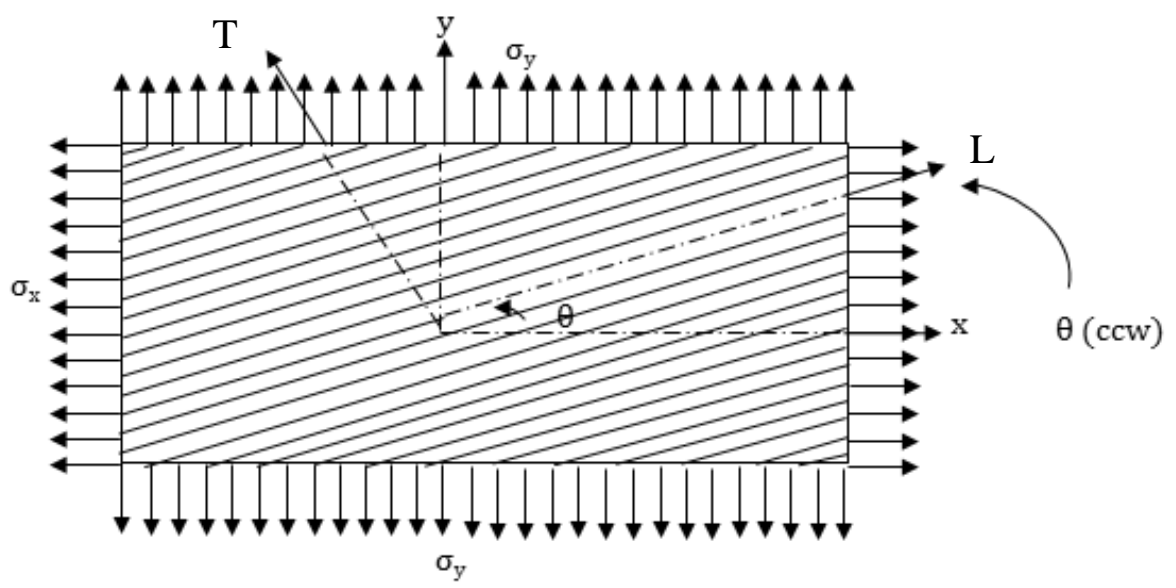

Figure 4-4 orthotropic lamina showing fiber angle, global to local coordinate system

The material properties of the fiber and matrix, used to calculate the material properties of the lamina and laminate, using the above equations in MATLAB code are given in Table 4-1. The MATLAB code used to find material properties of the lamina and laminate are given in Appendix A- 1 and the calculated values are given in Table 4-2. These properties are used to find Point and Average Stress Critera and variations in stress at and far from circular hole. The calculated material properties are also used to find the variations in strain and stress through the laminate thickness from Equation (20) and Equation (24) which is given in Appendix A-2 and Appendix A-3.

Table 4-1 Material - properties of fiber and matrix [133, 134]

\begin{tabular}{lcc}
\hline \multicolumn{1}{c}{ Parameter } & Carbon fiber T700 & M21 epoxy \\
\hline Elastic modulus (E) & $230 \mathrm{GPa}$ & $4.5 \mathrm{GPa}$ \\
Poisson ratio (v) & 0.25 & 0.40 \\
Volume fraction & $58 \%$ & $40.6 \%$ \\
Void & $1.4 \%$ & \\
Ultimate tensile strength & $17.35 \mathrm{MPa}(205 \mathrm{MPa})$ \\
Fiber diameter & $7 \mu \mathrm{m}$ \\
UD Hexply T700-M21GC stacking sequence & {$\left[ \pm 45^{\circ}\right]_{2 \mathrm{~S}}$} \\
\hline
\end{tabular}


Table 4-2 Calculated lamina and laminate material properties from MATLAB code

\begin{tabular}{|c|c|c|}
\hline Mechanical Properties & $\begin{array}{c}\text { Lamina } \\
\text { (GPa) axes }\end{array}$ & $\begin{array}{l}\text { Laminate } \\
\text { (GPa) axes }\end{array}$ \\
\hline Longitudinal Youngs modulus of the lamina $(G P a)=E_{L}$ & 134.8700 & 10.2501 \\
\hline Transversal Youngs modulus of the lamina $(\mathrm{GPa})=\mathrm{E}_{\mathrm{T}}$ & 8.1618 & 10.2501 \\
\hline $\begin{array}{l}\text { Poisson ratios of the lamina in Longitudinal } \\
\text { direction }=v_{L T}\end{array}$ & 0.3130 & 0.08546 \\
\hline $\begin{array}{l}\text { Poisson ratios of the lamina in Transversal direction } \\
=v_{T L}\end{array}$ & 0.0189 & 0.08546 \\
\hline Shear modulus of the fiber $(\mathrm{GPa})=\mathrm{G}_{\mathrm{f}}$ & 92 & - \\
\hline Shear modulus of the matrix $(G P a)=G_{m}$ & 1.2500 & - \\
\hline Transverse shear modulus of the lamina (in GPa) $=G_{L T}$ & 2.9214 & - \\
\hline Shear modulus of the laminate Gxy & - & 3.28113 \\
\hline
\end{tabular}

\subsection{Stress Concentration}

Stress concentration, usually called stress risers, are the places in a material where stresses are more concentrated than the rest of the material. These concentrated areas are due to holes, notches etc. in a material. Stress concentration locations are the places of crack initiation and propagation. Hence the life of a machine part largely depends on these locations, so the enhancement of a machine part life is related to minimizing these locations during machining. In the current study, the hole manufacturing techniques are under investigation to identify whether these stress risers sites are induced due to the manufacturing technique used (CHAPTER 6) or not, and by calculating the stress concentration to find a suitable machining process which produces less stress concentration sites. In this section a criterion will also be developed, based on the infrared thermographic stress and thermal behaviour. 


\subsubsection{Stress Concentration Criteria}

A circular hole at the center of an infinite orthotropic plate of radius $\mathrm{R}$ with origin of an $\mathrm{x}-\mathrm{y}$ axes system shown in Figure 4-5 and Figure 4-6. A uniform remote tensile stress $\sigma_{0}$ is applied parallel to the $y$-axis at the end of the plate. If the $\mathrm{x}$ and $\mathrm{y}$ axes are assumed normal to the plane of elastic symmetry, the normal stress $\left(\sigma_{\mathrm{y}}\right)$ distribution along the net section plane $\mathrm{x}$-axis in front of the hole in the direction of the $y$-axis at a distance $x$ from the circular hole of radius $\mathrm{R}$, has been shown by Lekhnitskii as [135];

$$
\sigma_{\mathrm{y}}(\mathrm{x}, 0)=\frac{\sigma_{0}}{2}\left[2+\left(\frac{\mathrm{R}}{\mathrm{x}}\right)^{2}+3\left(\frac{\mathrm{R}}{\mathrm{x}}\right)^{4}-\left(\mathrm{K}_{\mathrm{t}}-3\right)\left[5\left(\frac{\mathrm{R}}{\mathrm{x}}\right)^{6}-7\left(\frac{\mathrm{R}}{\mathrm{x}}\right)^{8}\right]\right]
$$

Where, $\mathrm{x}>\mathrm{R}$, $\mathrm{x}$ is the distance ahead of the hole and $\mathrm{K}_{\mathrm{t}}$ is the orthotropic stress concentration factor for an infinite width plate. For an isotropic material, the value of $\mathrm{K}_{\mathrm{t}}$ is equal to 3 and for the orthotropic stress concentration factor for an infinite width plate as determined from the following relationship [135];

$$
K_{t}=1+\sqrt{\frac{2}{A_{11}}\left(\sqrt{A_{11} A_{22}}-A_{12}+\frac{A_{11} A_{22}-A_{12}^{2}}{2 A_{66}}\right)}
$$

Where $\mathrm{A}_{\mathrm{ij}}$ are the elements of the orthotropic in-plane laminate stiffness matrix of the composite plate and can be determined from laminated plate theory as [130];

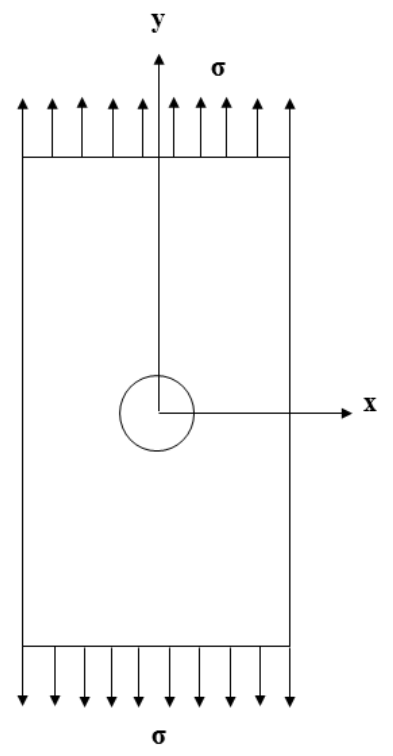

Figure 4-5 Orthotropic composite plate with circular hole 


$$
A_{i j}=\sum_{k=1}^{n}\left(\overline{Q_{i j}}\right)_{k}\left(h_{k}-h_{k-1}\right)
$$

and

$$
\begin{gathered}
\overline{Q_{11}}=Q_{11} \cos ^{4} \theta+Q_{22} \sin ^{4} \theta+2\left(Q_{12}+2 Q_{66}\right) \sin ^{2} \theta \cos ^{2} \theta \\
\overline{Q_{22}}=Q_{11} \sin ^{4} \theta+Q_{22} \cos ^{4} \theta+2\left(Q_{12}+2 Q_{66}\right) \sin ^{2} \theta \cos ^{2} \theta \\
\overline{Q_{12}}=\left(Q_{11}+Q_{22}-4 Q_{66}\right) \sin ^{2} \theta \cos ^{2} \theta+Q_{12}\left(\cos ^{4} \theta+\sin ^{4} \theta\right) \\
\overline{Q_{66}}=\left(Q_{11}+Q_{22}-2 Q_{12}-2 Q_{66}\right) \sin ^{2} \theta \cos ^{2} \theta+Q_{66}\left(\cos ^{4} \theta+\sin ^{4} \theta\right) \\
\overline{Q_{16}}=\left(Q_{11}-Q_{12}-2 Q_{66}\right) \sin \theta \cos ^{3} \theta-\left(Q_{22}-Q_{12}-2 Q_{66}\right)\left(\cos \theta \sin ^{3} \theta\right) \\
\overline{Q_{26}}=\left(Q_{11}-Q_{12}-2 Q_{66}\right) \sin ^{3} \theta \cos \theta-\left(Q_{22}-Q_{12}-2 Q_{66}\right)\left(\cos ^{3} \theta \sin \theta\right)
\end{gathered}
$$

where the values of $\mathrm{Q}_{11}, \mathrm{Q}_{22}$, etc, can be found from Equation (17).
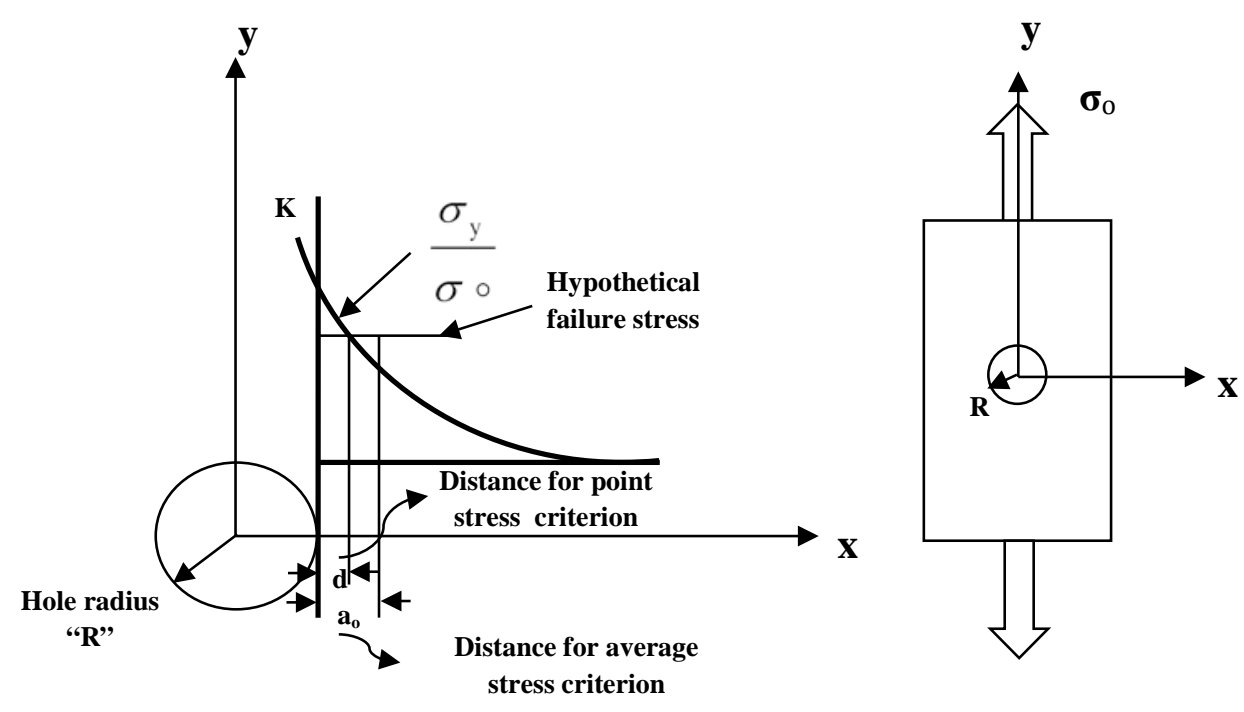

Figure 4-6 Failure mechanism of the circular hole specimen representing point and average stress criterion

So the unidirectional laminate $\mathrm{A}_{\mathrm{ij}}$ in Equation (26) may be replaced by $\mathrm{Q}_{\mathrm{ij}}$. In terms of effective elastic moduli in the principal laminate $\mathrm{x}$ - and y-axes, Equation 26 then becomes [130], 


$$
\mathrm{K}_{\mathrm{t}}=1+\sqrt{2\left(\frac{\mathrm{E}_{11}}{\mathrm{E}_{22}}-v_{12}\right)+\frac{\mathrm{E}_{11}}{\mathrm{G}_{12}}}
$$

Where $\mathrm{K}_{\mathrm{t}}=$ Stress concentration factor

and elastic constants for orthotropic plates are;

$E_{x}, E_{y}=$ Young's moduli in the $\mathrm{x}$ - and y-directions.

$v_{x y} \quad=$ In plane Poisson's ratio

$G_{x y} \quad=$ In plane shear modulus

For an orthotropic composite loaded in the longitudinal direction, Equation 29 becomes;

$$
\mathrm{K}_{\mathrm{t}}=1+\sqrt{2\left(\frac{\mathrm{E}_{\mathrm{L}}}{\mathrm{E}_{\mathrm{T}}}-v_{\mathrm{LT}}\right)+\frac{\mathrm{E}_{\mathrm{L}}}{\mathrm{G}_{\mathrm{LT}}}}
$$

Where $E_{L}, E_{T}, v_{L T}$ and $G_{L T}$ are elastic constants for the orthotropic composite. As a ply angle of \pm 45 was used, the axial modulus $\left(\mathrm{E}_{\mathrm{L}}\right)$ and the transverse modulus $\left(\mathrm{E}_{\mathrm{T}}\right)$ are equal. In other words, the ratio of the axial stiffness to the transverse stiffness in Equation (30) is unity. For calculating the orthotropic stress concentration factors of CFRP composite laminates, the Poisson ratio and the shear modulus are required. The values of these basic mechanical properties are listed in Table 4-2.

At the hole boundary $\mathrm{x}=\mathrm{R}$, the stress concentration factor from Equation (25) is given as [136];

$$
\mathrm{K}_{\mathrm{t}}=\frac{\sigma_{\mathrm{y}}(\mathrm{R}, 0)}{\sigma_{0}}
$$

Where $\sigma_{0}$ is the nominal or far field stress and $\sigma_{\mathrm{y}}$ is the maximum stress. As the circular hole size decreases, the stress concentration factor decreases and finally approaches unity (that is, a plate without a hole) [59].

To predict the failure modes of drilled composite laminates, various analytical criteria are developed. The main assumption that has been used is that there is a stress concentration in the vicinity of the damage area on the circular hole's drilled surface, which is a function of the material properties, specimen geometry and the applied loads. The criteria often used for calculating the strength of the specimens having circular holes are the Point Stress Criterion (PSC) and the Average Stress Criterion (ASC) proposed by Tercan M, and Whitney JM. [57, 58]. Both these criteria were calculated by Whitney and Nuismer from Lekhnitskii's Equation (25). The PSC assumes that failure occurs when the normal stress at some distance away from 
the circular hole, $d_{0}$, is equal to or greater than the unnotched laminate tensile strength or the tensile strength $\sigma_{\mathrm{f}}$ of the material. A comparable approach was introduced by Tercan [57], which assumed that failure occurs when the average stress over some distance ahead of the circular hole, $a_{0}$, equals the unnotched laminate strength as can be seen in Figure 4-6. An approximate solution in the form of a polynomial was used to obtain the normal stress distribution near to the hole in an infinite width plate. Considering these criteria, the stress around the circular hole in composite laminates was extensively studied by number of researchers [59, 119, 137], however for comparing two machining processes for its suitability, these criteria have not yet been studied. Details of these criteria are presented below;

\section{Point stress criterion}

The point stress criterion assumes that failure will occur when normal stress $\sigma_{y}$, at some fixed distance " $\mathrm{d}_{\mathrm{o}}$ " ahead of the circular hole along $\mathrm{x}$-axis direction, first reach the unnotched laminate tensile strength $\sigma_{\mathrm{f}}$, that is when [129];

$$
\sigma_{y}\left(R+d_{o}\right)=\sigma_{f}
$$

So the criterion of point stress can be obtained from Equation (25) and Equation (32), that is

$$
\begin{gathered}
\mathrm{K}_{\mathrm{point}}=\frac{\sigma_{\mathrm{y}}(\mathrm{x}, 0)}{\sigma_{\circ}}=\frac{1}{2}\left[2+\mathrm{p}_{1}^{2}+3 \mathrm{p}_{1}^{4}-\left(\mathrm{K}_{\mathrm{t}}-3\right)\left(5 \mathrm{p}_{1}^{6}-7 \mathrm{p}_{1}^{8}\right)\right] \\
\mathrm{PSC}=\mathrm{K}_{\mathrm{PSC}}=\left.\frac{\sigma_{\mathrm{y}}(\mathrm{x}, 0)}{\sigma_{\circ}}\right|_{\mathrm{x}=\mathrm{R}+\mathrm{d}_{0}}
\end{gathered}
$$

where

$$
p_{1}=\frac{R}{R+d_{0}}
$$

It may be noted that for very large holes, $p_{1} \rightarrow 1$. On the other hand, for vanishingly small hole sizes, $p_{1} \rightarrow 0$ and the ratio $\left(\sigma_{y} / \sigma_{\circ}\right) \rightarrow 1$, as would be expected [129]. 


\section{Average stress criterion}

This criterion assumes that failure occurs when the average values of $\sigma_{y}$, over some fixed distance $a_{\circ}$, ahead of the hole first reach the unnotched tensile strength $\left(\sigma_{f}\right)$ of the specimen, that is when [129];

$$
\frac{1}{a_{\mathrm{o}}} \int_{R}^{R+a_{\circ}} \sigma_{y}(x, 0) d x=\sigma_{f}
$$

From Equation (25) and Equation (35) we can obtain the ratio of "K", average stress criterion as,

$$
\begin{gathered}
\mathrm{K}_{\text {average }}=\frac{\sigma_{\mathrm{y}}(\mathrm{x}, 0)}{\sigma_{\circ}}=\frac{2-\mathrm{p}_{2}^{2}-\mathrm{p}_{2}^{4}-\left(\mathrm{K}_{\mathrm{t}}-3\right)\left(\mathrm{p}_{2}^{6}-\mathrm{p}_{2}^{8}\right)}{2\left(1-\mathrm{p}_{2}\right)} \\
\mathrm{K}_{\text {average }}=\left(\frac{1+\mathrm{p}_{2}}{2}\right)\left[2+\mathrm{p}_{2}^{2}+\left(\mathrm{K}_{\mathrm{t}}-3\right)\left(\mathrm{p}_{2}^{6}\right)\right] \\
A S C=K_{A S C}=\frac{1}{a_{0}} \int_{R}^{R+a_{0}} \sigma_{y}(x, 0) \cdot d x
\end{gathered}
$$

where

$$
p_{2}=\frac{R}{R+a_{\text {。 }}}
$$

The values of the characteristic distances $\mathrm{d}_{0}$ and $\mathrm{a}_{0}$ are determined by means of a curve fitting of the experimental data obtained from tensile tests of rectangular specimens containing holes of different sizes. Consequently, we used $\mathrm{a}_{0}=0.3 \mathrm{~mm}$ and $\mathrm{d}_{0}=0.72 \mathrm{~mm}$, resulting from the literature [59, 59, 119].

\subsubsection{IR Thermal and Stress concentration factor}

Stress concentration in an orthotropic laminate having a circular hole at the center of the specimen when uniaxial load was applied along the y-axis, as shown in Figure 4-6, is calculated in this section. In this case the maximum stress is the circumferential stress around the circular hole along the loading direction (at $\theta=90^{\circ}$ ). Stress concentration around the hole can be calculated from the following equation [136];

$$
K_{\text {exp }}=\frac{\sigma_{\max }}{\sigma_{\text {far field }}}
$$

The above equation can also be written as; 


$$
S C F_{I R}=K_{I R-S t r e s s}=\frac{\Delta \sigma_{\max }}{\Delta \sigma_{。}}
$$

Where, $S C F_{I R}$ is the stress concentration factor obtained from an IR camera's stress analysis, $\sigma_{\max }$ is the maximum stress at hole boundary, $\sigma_{0}$ is the nominal (far field) stress.

The stress concentration factor is dependent on the machined surface and on fatigue loading. As we know, the deformation of solid materials is almost always accompanied by releases of heat. When the material becomes deformed or is damaged and fissured with the increasing load, a part of the energy necessary to start and to propagate the damage is transformed in an irreversible way into heat as discussed in Section 3.4. So to find the stress concentration factor of the two types of specimens, the temperature parameter can be included in Equation (41). As we know, the thermo-elastic equation utilized by the IR camera to generate the stress fields is [138];

$$
\Delta \sigma=\Delta \mathrm{T} \rho \mathrm{C} /(\alpha \mathrm{T})
$$

where $\Delta \mathrm{T}$ is the temperature change sensed by the IR camera, $\rho$ is the specimen's material density, $C$ is the specimen's specific heat capacity, $\alpha$ is the specimen's coefficient of thermal expansion, and $\mathrm{T}$ is the ambient temperature sensed by the IR camera. This equation assumes adiabatic conditions, that is, there is no significant heat loss. The values inputted into the IR software for $\mathrm{CF} /$ Epoxy material were $\rho=1.44 \mathrm{~g} / \mathrm{cm}^{3}, \mathrm{C}=1040 \mathrm{~J} /(\mathrm{kgK})$, and $\alpha=2.6 \times 10^{-6}{ }^{\circ} \mathrm{C}^{-1}$. When the specimen's material density, specific heat capacity, coefficient of thermal expansion and the ambient temperature sensed by the IR camera was considered constant, then the change in stress is a function of change in temperature as can be seen from Equation 42. So a change in maximum and far field temperature is the quantitative measure of the respective stresses. Accordingly Equation 40 becomes;

$$
\begin{gathered}
\mathrm{K}_{\text {thermal }}=\frac{\Delta \mathrm{T}_{\max } \rho \mathrm{C} /(\alpha \mathrm{T})}{\Delta \mathrm{T}_{\text {far field }} \rho \mathrm{C} /(\alpha \mathrm{T})}=\frac{\Delta \mathrm{T}_{\max }}{\Delta \mathrm{T}_{\text {far field }}} \\
\mathrm{TCF}_{\mathrm{IR}}=\mathrm{K}_{\text {IR-Thermal }}=\frac{\Delta \mathrm{T}_{\text {max }}}{\Delta \mathrm{T}_{\mathrm{o}}}
\end{gathered}
$$

Where, $T C F_{I R}$ is the thermal concentration factor obtained from the IR camera's temperature reading, $\Delta T_{\max }$ is the difference between the maximum and the minimum temperatures $\left(T_{\max }-T_{\min }\right)$ on hole boundary, $\Delta T_{0}$ is the difference between the far-field maximum and minimum temperatures $\left(T_{\max }-T_{\min }\right)$. 
The Point stress criterion (PSC) and the average stress criterion (ASC) proposed by Tercan and Whitney $[57,58]$ as well as the stress concentration factor derived from the IR thermal and stress analyses were calculated and compared to investigate whether the type of machining affects these stress criteria or not. The expressions of each stress criterion was calculated analytically from Equation (33) for the PSC, Equation (38) for the ASC and experimentally from Equation (40) and Equation (43) using stress and thermal values obtained from an IR camera and the calculated values are given in Table 4-3.

Table 4-3 Effect of machining process on the stress concentration factors and strength of carbon/epoxy composite with circular hole under uniaxial tensile loading

\begin{tabular}{lcc}
\hline \multicolumn{1}{c}{ Description } & $\begin{array}{c}\text { Thermal and Stress } \\
\text { Concentration factor }\end{array}$ & Remarks \\
\hline Point stress criterion & 1.95 & $\mathrm{~d}_{0}=0.72 \mathrm{~mm}$ \\
& & $\mathrm{a}_{0}=0.3 \mathrm{~mm}$ \\
Average stress criterion & 1.87 & \\
$\begin{array}{l}\text { Thermal concentration factor for CM specimen } \\
\text { (Altair, temperature) }\end{array}$ & $2.29 \pm 0.06$ & \\
$\begin{array}{l}\text { IR stress concentration factor for CM specimen } \\
\text { (Altair LI, stress) }\end{array}$ & $2.39 \pm 0.15$ & \\
$\begin{array}{l}\text { Thermal concentration factor for AWJM } \\
\text { specimen }\end{array}$ & \\
\hline $\begin{array}{l}\text { IR stress concentration factor for AW } \\
\text { specimen }\end{array}$ & $1.93 \pm 0.03$ \\
\hline
\end{tabular}

It is evident that the influence of the machining process on the strength in the CM specimen was more pronounced than in the AWJM specimen. For the CM specimen the stress concentration factor was high $(2.39 \pm 0.15)$ showing larger stress riser locations at the machined surface. Therefore, the CM specimens failed earlier than the AWJM specimens. In addition, stress 
concentration values for the CM were also $14.85 \%$ and $18.35 \%$ greater than the values calculated from the point and average stress criterion, respectively. In the case of the AWJM specimens, the stress concentration value was close to the analytically calculated values for PSC (1.95) and ASC (1.87) which shows that the surface created in the AWJM process produced less stress concentration sites and is more close to the theoretical conditions. Thermal concentration values of CM specimens were over-estimated by $15.70 \%$ compared to the AWJM specimens.

To investigate the reason for this difference in the behaviour of the CM and the AWJM specimens further microscopic analysis is needed and is presented in CHAPTER 5 to CHAPTER 7.

\subsection{Stress field at and far from the circular hole}

The stress field around a circular hole were calculated from the strains values in xx, yy and xy directions obtained from experiments and will be validated with Lekhnitskii's equation (Equation 25) in this section.

Considering local variations in the mechanical properties are neglected, and under plane stress condition the overall mechanical properties of the composite specimen in the on-axis tensile loading are assumed to be orthotropic, then axial stresses around the circular hole can be obtained from the experimentally measured strain field $\left(\varepsilon_{x x}, \varepsilon_{y y}\right)$ using the Equation (44) [59];

$$
\sigma_{x x}=\frac{E_{x x} \varepsilon_{x x}}{\left(1-v_{x y}^{2}\left(E_{y y} / E_{x x}\right)\right)}+\frac{v_{x y} E_{y y} \varepsilon_{y y}}{\left(1-v_{x y}^{2}\left(E_{y y} / E_{x x}\right)\right)}
$$

The theoretical stress was calculated using Equation (25) and the stress distribution at different locations along the width of the specimens; obtained using Equation (44) for the applied load of $7 \mathrm{kN}$ is presented in Figure 4-7. Figure 4-7 shows that for all specimens, stresses are higher at and near the edge of the circular hole and then a sharp decrease in the stresses was noted near the edge of the hole, however, it deceases gradually when moving away from circular hole along the width of the specimen and was almost uniform towards the edge of the specimen. This higher stress at one location than the rest of the specimen also caused the early failure of the specimen. Another observation is also noted that near the hole, stresses calculated from the experimental strain values are lower than the stresses obtained from the analytical analysis using Equation (25). 
Figure 4-7 illustrated that for the CM specimen, the stress at the hole boundary is higher (around 16\%) than the AWJM specimen. A higher stress concentration in the CM specimen was produced from the strain field $\left(\varepsilon_{x x}, \varepsilon_{y y}\right)$ around the hole. This difference in the CM and AWJM specimens probably owed to the circular hole machined surface generated due to the different machining process. Stresses far from the hole edge were uniform, which indicate that there was no abnormality in and outside the specimen and only the circular hole's machined surface was responsible for the difference in the stress values. To identify the reason for this difference in the mechanical behaviour of the two machining processes, a further analysis is indeed needed. For this purpose static and fatigue tests under tension-tension loading condition were performed and are presented in the CHAPTER 5 to CHAPTER 7.

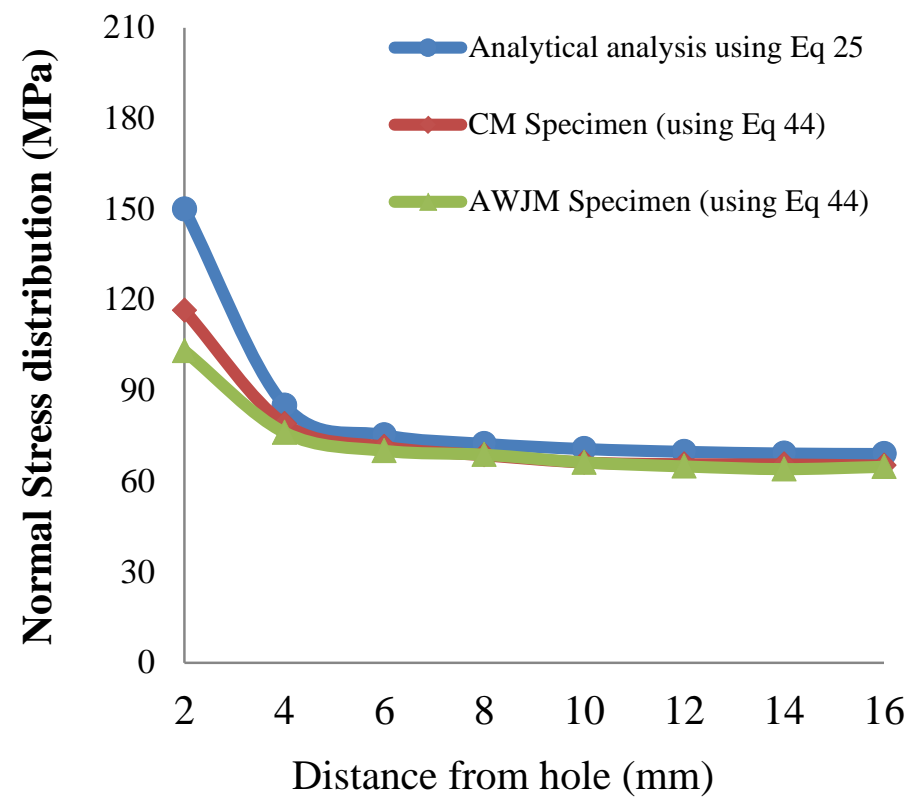

Figure 4-7 Stress distribution of CFRP composites with circular hole subjected to a tensile load along long axis of the specimen.

\subsection{Summary}

The difference in the stress-strain behaviour, IR thermal and stress concentration factors between conventional and non-conventional drilled specimens were mainly attributed to the machining process utilized for drilling holes. The IR thermal concentration factor was developed from the IR stress equation. It was found that IR thermal and stress concentration factors of the CM 
specimens were over-estimated by $15.70 \%$ and $16.30 \%$ respectively, compared to the AWJM specimens. This in turn reduces the mechanical properties of the CM specimens.

The difference in machined surface texture was responsible for the initiation of stress concentration sites as evident from the IR camera's thermal and stress analysis and stress field at and far from the circular hole, which showed a clear difference between the specimens drilled with conventional and non-conventional machining processes. To explore this difference in the mechanical behaviour, further experiments are indeed needed, which will be investigated by conducting static tests, a surface microstructure analysis and fatigue analysis.

All observations of this study indicated that the choice of the process of machining has an important role on the mechanical properties of the structural components. 


\section{CHAPTER 5. STATIC TEST RESULTS FOR THE SINGLE PLATE}

The current chapter will focus on static test results to find the strength, stiffness and ultimate tensile strength of a structural part. First, the static test results will be presented and discussed followed by the analysis of strain maps obtained from a CCD camera. At the end of chapter, a brief summary of the chapter will be presented.

\subsection{Static Test Analysis}

This section presents experimental results of the CM and the AWJM specimens made from UD prepreg subjected to tensile static loading using an Instron 4206 tester, to determine the behaviour of the load on the machined hole and its vicinity. Based on the experimental tests the results and discussion of the stress-strain response is presented in following.

\subsubsection{Results and discussion}

The stress vs strain curves for the CM and AWJM specimens are graphically shown in Figure 51. It was observed that, for all specimens the stress-strain curves passed through three phases: The first phase started when the static load was applied, initially higher stress yields lesser strain as shown by the linear elastic phase, a straight line, which is characterized by an elastic modulus $\mathrm{E}_{0}$. In this phase, the specimens starts to undergo matrix microcracking which is due to the large increase in the stress values. After the elastic limit the specimen underwent further microcracking in the second phase. In this phase, the slope of the curve decreased, reflecting the beginning of the elastic to plastic behaviour. A possible cause of this deviation from linearity was damage initiation resulting from strain concentration in the vicinity of the hole, which was slightly larger for the CM than the AWJM specimen. The degradations by shearing was dominating and this non-linearity was due to matrix ductility. The matrix continues to crack and the load was transferred progressively to the fibers after the non-linearity appears in the stressstrain curves.

For all specimens, the crack initiated at a value which was below the ultimate stress. When the specimen was further stressed, it resulted in the breakup of fibers as it reached a critical value. In the last phase, stress decreased until the final break. The failure load could be considered as the 


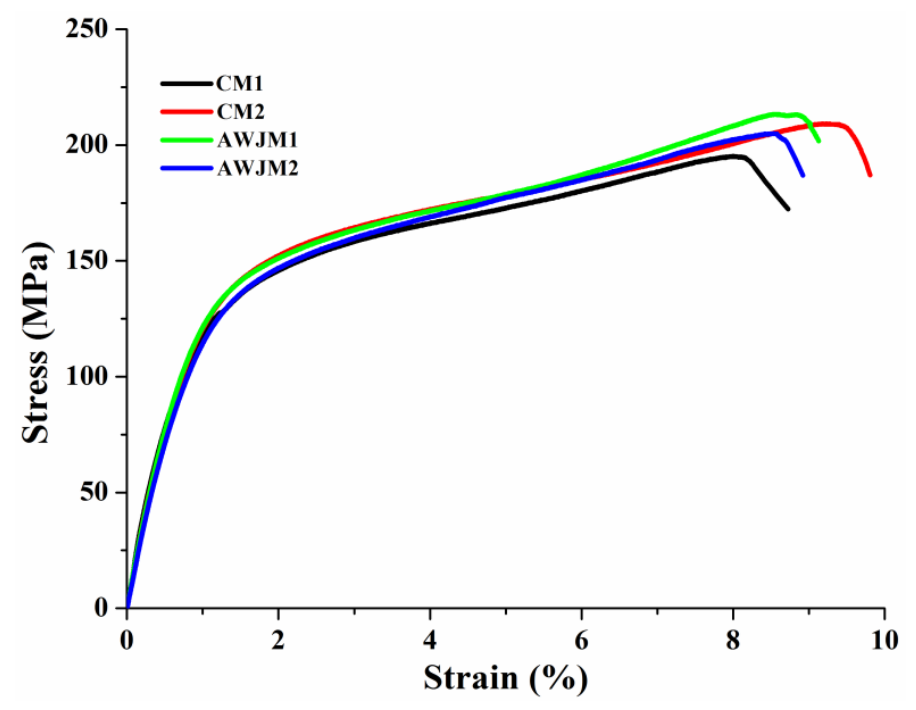

Figure 5-1 Static tests strain values from stress vs. strain graph for the laminates drilled with abrasive water-jet and conventional machining process

load at which material deviates from the non-linear stress-strain response. A sudden fracture in the vicinity of the hole was observed for all specimens. For the CM and AWJM specimens the average failure stress was $202 \pm 7 \mathrm{MPa}$ and $209 \pm 4 \mathrm{MPa}\left(0.21 \mathrm{kN} / \mathrm{mm}^{2}\right.$ or $\left.17.35 \mathrm{kN}\right)$ and the strain measured showed almost the same behaviour, which was $8.79 \pm 0.62 \%$ and $8.70 \pm 0.1 \%$, respectively. Failure stress for AWJM was 6.70\% higher than the CM specimen. All specimens showed failure due to the stress concentration near the hole. There was no significant change in the maximum stress per unit area (2.35 and 2.45 average for CM and AWJM specimens respectively) and the ultimate strength of the both specimens. All specimens showed that the machined hole surface controls the failure stress rather than any other abnormality far from the hole. It was found that the average ultimate tensile strength of all the tested specimens $\left(\left[ \pm 45^{\circ}\right]_{2 \mathrm{~S}}\right)$ were $17.35 \mathrm{kN}$, irrespective of the drilling process used.

\subsection{CCD Camera Analysis}

\subsubsection{Cartographic Analysis for Strain}

The damage mechanism of CM and AWJM specimens using images obtained from a CCD camera is described in this section. From these images strain distributions can also be investigated, which will be helpful to find stresses and strain concentrations around the holes. From the strain concentration we can find the stress concentration and consequently the effect of the machined surface texture created due to the different machining processes. 
The digital images of strain distribution measured along the longitudinal direction OY and transverse direction $\mathrm{OX}$ of the specimens under an axial tensile loading of $14 \mathrm{kN}$ is presented in Figure 5-2 and Figure 5-3, respectively. The cartography of the deformations $\varepsilon_{\mathrm{xx}}, \varepsilon_{\mathrm{yy}}$ and $\varepsilon_{\mathrm{xy}}$ was obtained from the CCD camera system. The captured area around the circular hole is shown in these digital images are to observe the influence of the drilling process on the behaviour of the composite laminate under tensile load before failure. The strain field shows high strain values around the hole indicating the non-uniformity as shown in these figures. In Figure 5-2a and Figure 5-3a, a positive strain along a longitudinal axis OY is present everywhere and a maximum value is near the edge of hole. In the area above and below the hole, strain values are more than in the small axis, where fibers are oriented in between the hole edge and the small width perpendicular to the axial loading direction. Consequently, negative values are obtained along the transverse axis OX as shown in strain field images (Figure 5-2b and Figure 5-3b).

These strain results were caused by the Poisson's effect. From all the images it can be seen that the strain concentration is propagated parallel to the fiber directions and makes a pattern similar to the fiber direction which is $\pm 45^{\circ}$. However, for the CM specimens the crack initiation can be seen in $-45^{\circ}$ fiber direction. A common trend of elongation of the hole in the direction of the applied tensile load OY is also observed in all specimens. At $160 \mathrm{MPa}$ the maximum strain $\left(\varepsilon_{\mathrm{yy}}\right)$ was $3.41 \%$ and $3.74 \%$ for CM and AWJM specimens respectively. From the digital images correlation it was observed that the strain $\left(\varepsilon_{\mathrm{yy}}\right)$ in the AWJM specimen is $8.82 \%$ higher than the CM specimen.

\subsection{Summary}

In static tests, the stress was correlated with the strain values and it was found that the point at which a crack was initiated the stress-strain curve became nonlinear. Test results showed that the static tensile testing did not indicate any significant difference in the mechanical response between conventional and abrasive water-jet machined specimens, that is, strength and stiffness, as both specimen types had almost similar ultimate tensile strengths and strain values. Static test results do not provide a significant difference between two machining processes and do not single out a more structurally resilient component. The specimens machined with the conventional drill bit withstand a comparatively lower force than the hole machined with the AWJM process. From the static test results it is evident that the machining processes have no 


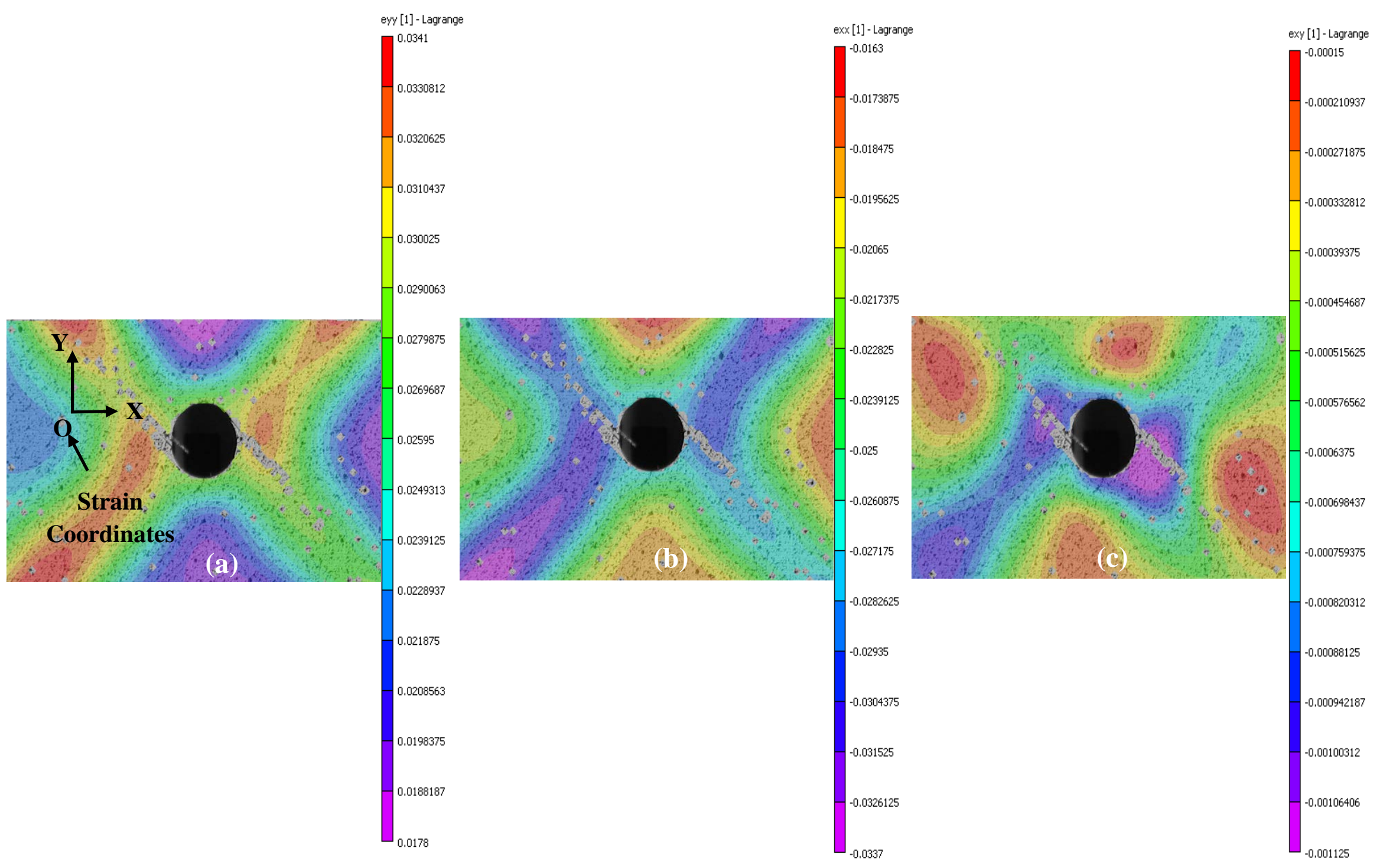

Figure 5-2 Digital images showing: (a) strain coordinates, and specimen showing cartography of the deformations in plan YY ( $\left.\varepsilon_{\mathrm{yy}}\right)$, (b) $\mathrm{XX}\left(\varepsilon_{\mathrm{xx}}\right)$ and (c) XY ( $\left.\varepsilon_{\mathrm{xy}}\right)$ under axial tensile load of $14 \mathrm{KN}$ in the direction of OY for CM specimen 


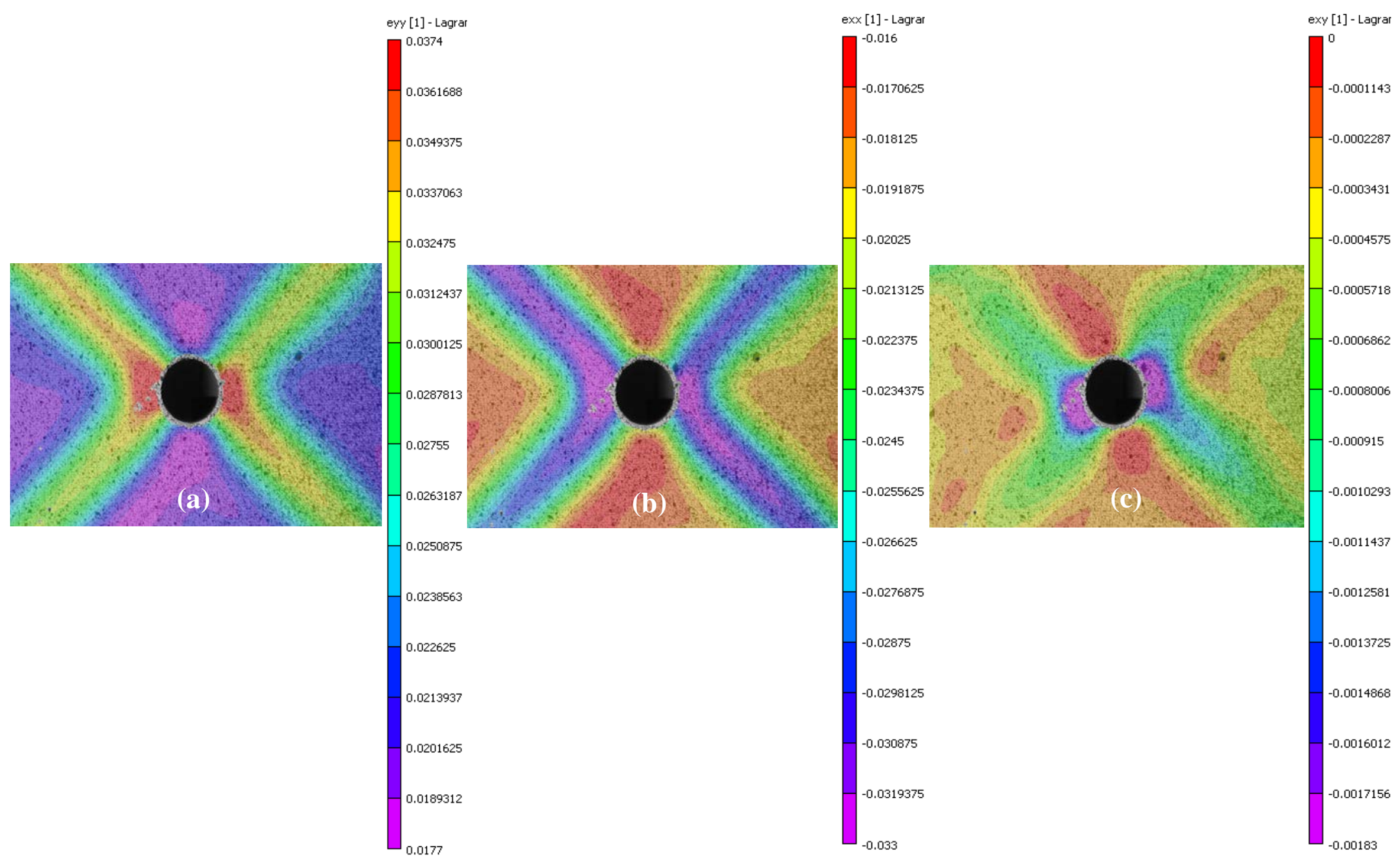

Figure 5-3 Digital images showing deformations in plans (a) YY ( $\left.\varepsilon_{y y}\right)$, (b) XX ( $\left.\varepsilon_{\mathrm{xx}}\right)$ and (c) XY ( $\left.\varepsilon_{\mathrm{xy}}\right)$ under axial tensile load of $14 \mathrm{KN}$ in the direction of OY for AWJM specimen. 
effect on the performance of carbon-epoxy composite laminates. So it is concluded that there is a need of an alternating experimental technique to obtain progressive damage evolution to assess the influence of the machining process on the surface of the circular hole. For this purpose, all of the structural parts and assembly specimens will be tested against fatigue cyclic tensile load to examine the damage propagation. 


\section{CHAPTER 6. FATIGUE TEST RESULTS FOR THE SINGLE PLATE}

The current chapter will focus on the damage detection using the local and global detection techniques during fatigue tests of a structural part. In the first section, reduction in elastic modulus during fatigue tests will be presented. In the following section, the damage evolution of the laminates having different stacking sequence tested with different tests parameters will be presented followed by the analysis of the surface microstructure of the pre-fatigue specimens using a surface roughness profilometer and scanning electron microscopy. In the third section, surface temperature recorded from IR thermography during cyclic tensile tests will be analyzed. The change in surface temperature will further be correlated with the damage evaluation. In this way the investigations of the local damage detection technique (extensometer) can also be validated with the global damage detection technique (IR thermography investigation). In section four, the endurance limit results will be discussed followed by the development of thermographic damage criterion. In the next section, the failure mechanism of all fractured laminates will be discussed using scanning electron microscopy. At the end of the chapter a brief summary of all tests results will be presented.

\subsection{Fatigue Analysis}

\subsubsection{Elastic Modulus Results and Discussion}

The elastic modulus was determined for all specimens tested at different step loads. It was calculated from the slope of the linear part of the stress-strain curves at each loading cycle. The stress was calculated from the axial force and initial cross sectional area whereas the resulting strain was measured by the Instron testing machine.

The graphs plotted in Figure 6-1 show the changes in the elastic modulus for all specimens under tensile cyclic loading from 3 to $11 \mathrm{kN}$. The elastic modulus that decreased during the test was plotted against the number of cycles for all specimens. It must be noted that in all figures the fatigue cycles were normalized $\left(\mathrm{N} / \mathrm{N}_{\mathrm{f}}\right)$ by dividing the initial cycle number by the number of cycles to final failure. The failure of all the specimens was measured at a specified elastic modulus and number of loading cycles.

Figure 6-1 shows a clear trend of stiffness degradation with an increasing number of cycles $\left(\mathrm{N} / \mathrm{N}_{\mathrm{f}}\right)$. For the load-controlled fatigue test, Figure 6-1 shows that stiffness was initially almost 

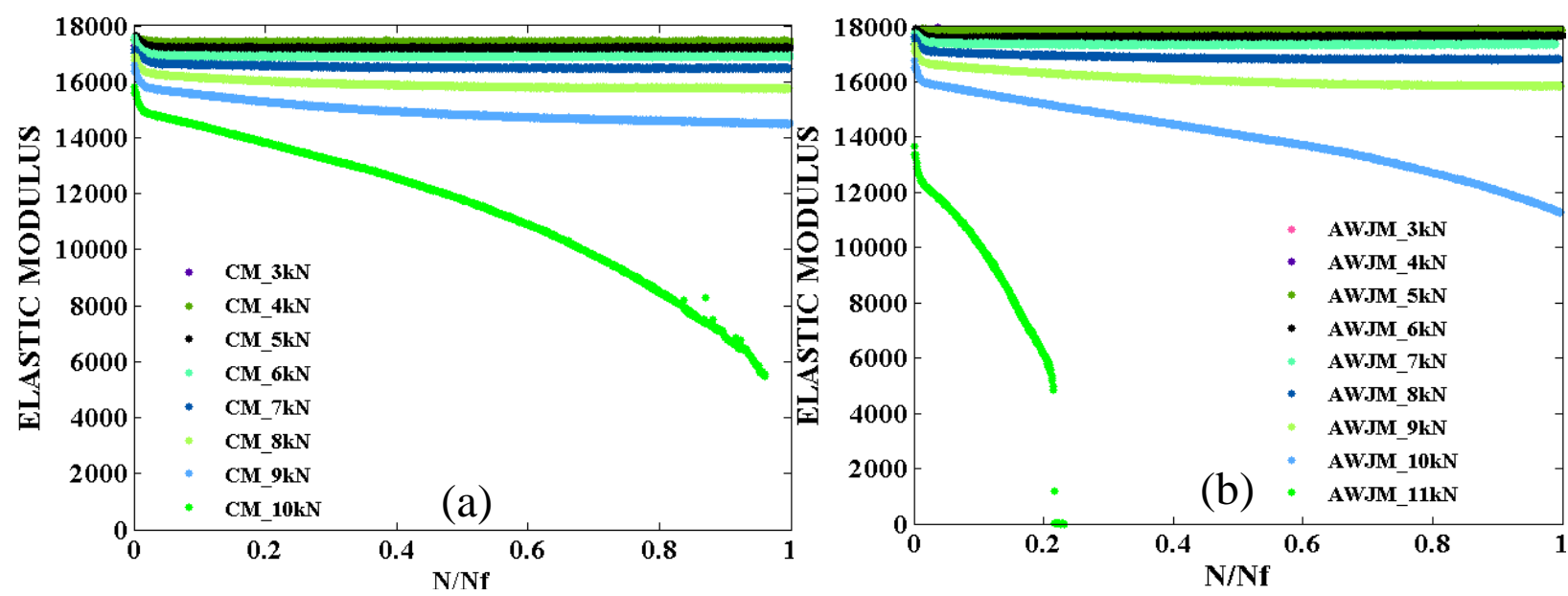

Figure 6-1 Calculated experimental tests results of the elastic modulus vs. normalized cycles for fatigue loading from $3 \mathrm{kN}$ to rupture of all (a) CM and (b) AWJM specimens. All units are in Megapascal.

the same for all specimens of the CM and the AWJM process. A loss of stiffness at the same loading stage (46\% of UTS) was 4\% more in CM than AWJM specimens. This difference in stiffness degradation was increased from $4 \%$ to $10 \%$ when the load was increased to $53 \%$ of UTS. It is important to note that a further increase of the load resulted in the failure of CM specimens at a loading of $59 \%$ of UTS $(10 \mathrm{kN})$. However, a further increase in the load to $59 \%$ of UTS resulted in 28\% of stiffness loss in the AWJM specimen when compared to the loss of stiffness which was noted in same specimen at the previous loading step (53\% of UTS) (Figure 6-2). In this case, the sudden stiffness loss was noted at a load of $65 \%$ of UTS, when the specimen failed.

Figure 6-1 shows a loss of stiffness in three different stages. Crack initiation and propagation in these three stages can be easily understood from Figure 6-3 [139]. The first microcrack developed in the matrix in the areas of voids, machining defect areas which are evident from the sharp increase in the damage curve. This stage is up to $10 \%$ of the total fatigue life. These microcracks grow with the increase of the cyclic load and at the interface of the matrix/fiber it changes its direction because of the high strength of the fiber. Crack propagation is continuous at the interface which also created stress concentration in that region. With a further increase in the load, stress concentration increases and finally breaks the fiber as shown from sudden decrease in stiffness in stage III (Figure 6-1) and sudden increase in damage (Figure 6-3) [110]. For CM specimens, this failure phenomenon was combined with the initial machining 

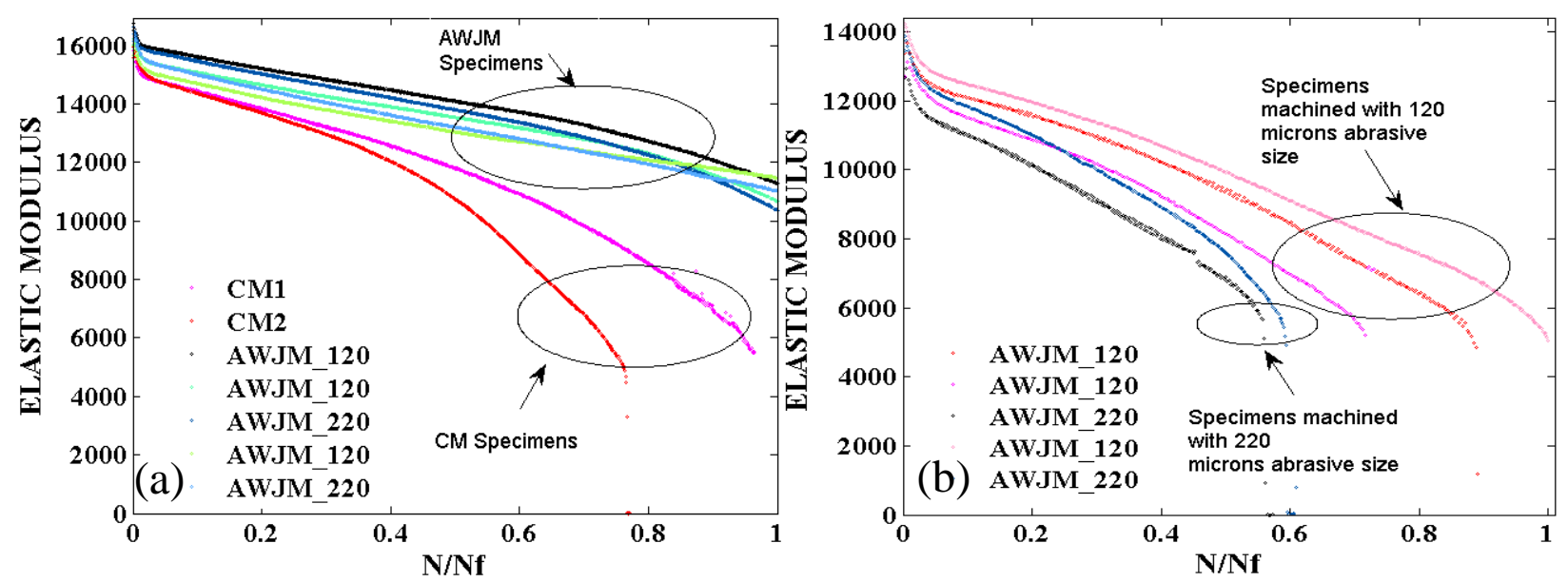

Figure 6-2 Calculated experimental tests results of the elastic modulus vs. normalized cycles for fatigue loading cases of (a) 53\% and (b) 59\% load to rupture of all specimens. A similar trend was also obtained for all other fatigue loading cases of specimens drilled with conventional and AWJ machining. All units are in Megapascal.

induced damage which increases the damage rate of these specimens and resulted in the early failure of the CM specimens when compared with AWJM specimens.

Besides the stiffness loss and the failure of AWJM specimens at a load of 65\% of UTS, Figure 62 also shows the effect of the abrasive particle size on the loss of stiffness. Composite laminates drilled with AWJM process used abrasive sizes of 120 and $220 \mu \mathrm{m}$ for machining holes. Figure 6-2 shows that AWJM specimens drilled with both types of abrasive sizes, failed at 65\% of load of UTS, however, a different fatigue life was observed for the specimens drilled with both types of abrasives. When abrasive of a large particle size was used we noted 18 - 40\% decrease in fatigue life than the specimens drilled with the small abrasive particles in the same loading stage. This shows that the large particle size created more stress concentration sites which was

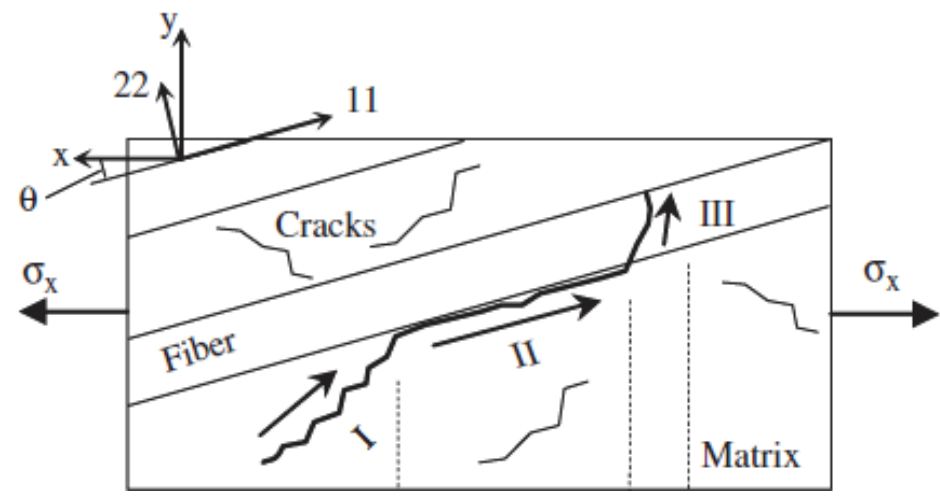

Figure 6-3 Three regions of cracking propagating mechanism in composite laminate [139] 
responsible for the early failure of the specimens. Nevertheless, a large abrasive size deteriorates the machined surface more than a small size on one side, but on the other side, a large abrasive size increases the material removal rate. To confirm and to investigate the reason of the difference in stiffness loss of the two types of specimens, further analysis was carried out and is presented in following section.

\subsection{Damage Analysis}

\subsubsection{Damage assessment (Angle ply Laminate)}

The change in stiffness degradation curves during cyclic loading is commonly used to quantify the damage in the unidirectional FRP composite laminates. The damage accumulation (D) is related to the change in the ratio of dynamic stiffness $\left(E_{i}\right)$ to the static stiffness $\left(E_{0}\right)$ and can be calculated from Equation (1).

Several fatigue tests were performed at various loading steps starting from $3 \mathrm{kN}$ with the increase of $1 \mathrm{kN}$ in each step, and for each step the damage growth was calculated by using Equation (1). Figure 6-4 (Appendix A-6 and Appendix A-7) plots the histories of the damage assessment versus the normalized cycles $\left(\mathrm{N} / \mathrm{N}_{\mathrm{f}}\right.$ where $\left.\mathrm{N}_{\mathrm{f}}=5000\right)$, whereas Figure 6-5 plots the histories of damage progression (damage vs normalized cycles) for a cyclic loading amplitude of
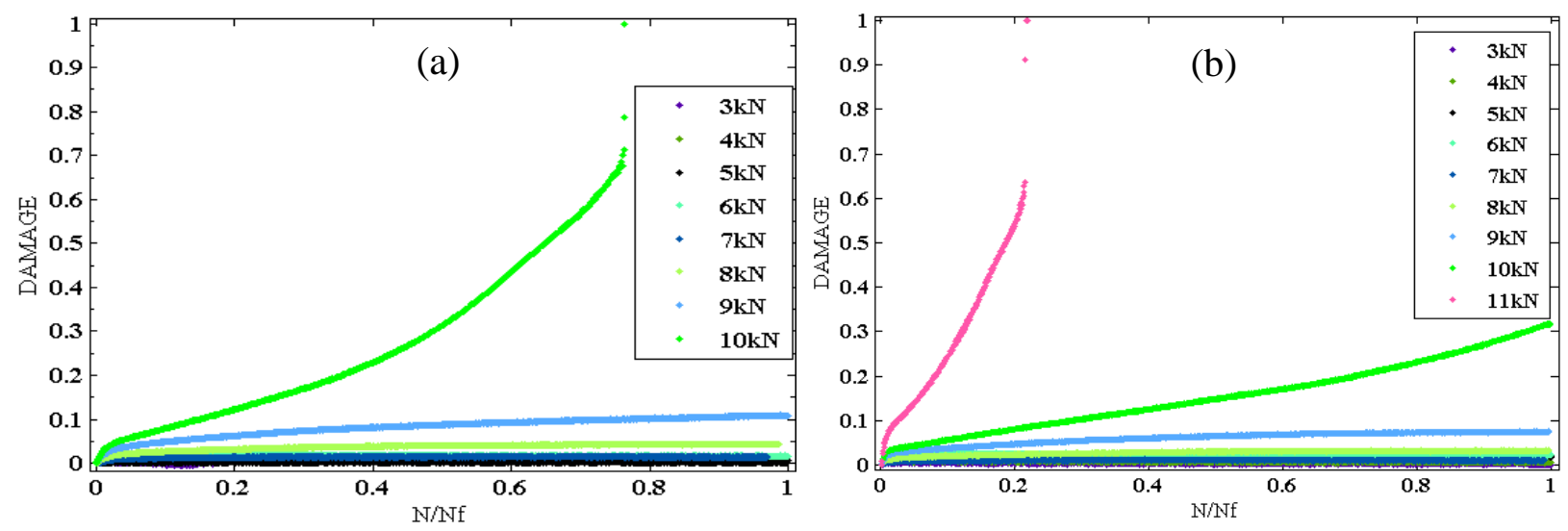

Figure 6-4 Comparison of the evolution of the damage for various level of loading for (a) Conventional machining and (b) Abrasive water-jet machining

$10 \mathrm{kN}$ (59\% of UTS) and $11 \mathrm{kN}$ (63\% of UTS) for all tested specimens. Data presented in these figures have been normalized $\left(\mathrm{N} / \mathrm{N}_{\mathrm{f}}\right.$, where $\mathrm{N}_{\mathrm{f}}=5000$ cycles). In the fatigue life cycles, up to 8 $\mathrm{kN}$ (47\% of UTS) and $9 \mathrm{kN}$ (53\% of UTS), the fatigue damage increased gradually with the 
increase in the load for CM and AWJM specimens, respectively, and the damage was less than $5 \%$. At these load levels, the behaviour of the two types of specimens are almost similar because of the continuous decrease of the mechanical properties, showing almost linear behaviour. When the load reaches $9 \mathrm{kN}$ (53\% of UTS) the cumulative damage does not exceed $10 \%$ for the CM specimens. In tests conducted at $10 \mathrm{kN}$ (59\% of UTS) shown in Figure 6-4a, the CM specimens ruptured after less than 3900 cycles $(\mathrm{N} / \mathrm{Nf}=0.8)$, accumulating damage levels was between 50$66 \%$. Under the same loading, after the same number of cycles, the AWJM specimens continued to withstand cyclic stresses and the accumulated damage was only 20\% (a difference of 30 percent when compared to the CM). The AWJM specimens lasted all 5000 cycles without rupturing, accumulating a maximum damage level of only 33\%. Finally, the AWJM specimen ruptured at $65 \%$ load of UTS. These results indicate that for any given load stage, AWJM specimens display higher resistance to fatigue damage than CM specimens.
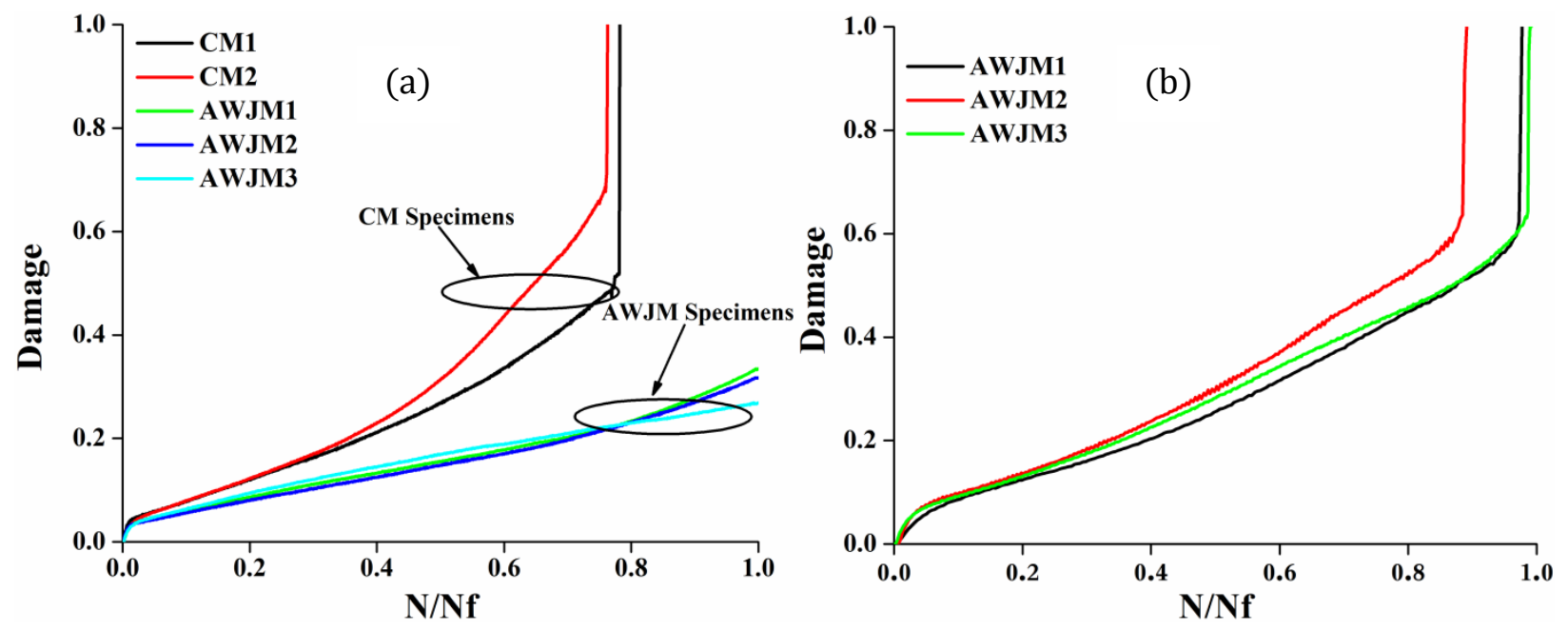

Figure 6-5 Comparison of the evolution of the damage for fatigue loading cases of (a) $59 \%$ and for (b) $65 \%$ load to rupture, for all examined specimens.

As the severity of the damage increases, a decrease in stiffness was also detected from the larger reduction in the elastic properties, as evident from Figure 6-1 and Figure 6-2. The experimental results of damage growth presented in Figure 6-4 (Appendix A-6 and Appendix A-7) show that there are three different stages during the damage evolution in composite laminate. These stages were explained in Section 6.1.1. 
In literature, only few studies are interested in identifying the influence of damage induced by the process of machining on the mechanical behaviour. However, these studies only considered conventional machining such as axial or orbital drilling. For instance, Davim et al. [140] found that during the drilling of a composite part made of carbon fiber reinforced plastics, the damage area increased when the cutting speed was increased from 50 to $70 \mathrm{~m} / \mathrm{min}$. In addition, it was also mentioned that the use of digital analysis was suitable for the evaluation of the damages induced by machining. In the same thematic, Ghidossi P. et al. [64] investigated the influence of cutting parameters on the damage development and mechanical response of off-axis glass/epoxy unidirectional coupons drilled with conventional machining. The off-axis tensile failure stresses of glass/epoxy composite specimens were significantly influenced by the machining parameters. In the research work conducted by [28] it was observed that the damage development depends on the tool geometry and feed rate. Rakesh et al. [141] examined GFRP laminates with drilled holes. It was observed that composite laminates failed around the circular hole under tensile load. The reasons were the matrix cracking and shear failure.

Furthermore, various researchers identified the root cause of the failure of the drilled composite during fatigue loading. Fatigue damage on a machined surface largely depends on the residual stresses, mechanical properties of the material, microstructure and on the stress concentration sites initiating from the surface topography. Surface irregularities generated by machining processes also play an important role in damage initiation and then propagation. Generally, the fatigue strength of a machine part decreases when the surface roughness increases [142]. In [84], it was identified that the surface roughness $R a$ is generally responsible for defining surface quality. Furthermore, it was identified that surface roughness is inversely proportional to the machined surface quality. A similar observation was also reported by [73], relating the fatigue life directly with the surface finish. It was found that fatigue strength was related to the surface irregularity depth. In the same thematic, Bayoum M.R. et al. [80] investigated the effect of a machined surface on the fatigue life. The fatigue life was found decreasing when more microcracks and micro voids were found on the surface, which was due to the increase in surface roughness. Furthermore, it was found that the increase of the surface roughness decreases the crack propagation life when the fatigue load was applied [80]. These microcracks act as crack nucleating sites with the increase in cyclic loading, which decrease the fatigue strength of the machine part [143]. A similar conclusion was also obtained by Taylo D. et al.[144]. It was 
observed that the machine surface generated with the conventional machining process resulted in a decrease in fatigue properties compared to the polished surface which is generally used for laboratory specimens. The decrease in the fatigue strength of the conventional machined specimen was strongly related to the roughness of the machined surface obtained from this process. Since fatigue is a surface sensitive testing method, when the cyclic load is applied to a machine part, crack nucleation starts from the free surface. Hence, a good fatigue life mainly depends on the roughness of the machined surface. Therefore surface roughness is considered as the most significant factor affecting the fatigue life [80]. All these previous research works shows that surface roughness is the only factor that affects fatigue life. Moreover, Griffiths [73] extensively studied the relation between the functional performance and surface integrity by taking into account the influence of the surface profile parameters on fatigue, stress and the fracture of the components, as shown in Table 6-1.

To investigate the reason for the different damage accumulation behaviour of the current study, a further analysis is needed to appreciate this difference between the two processes. Since it was identified from the literature that fatigue is surface sensitive loading application and surface roughness is mainly responsible for crack nucleation, so we analyzed the damage behaviour with surface roughness and SEM images, which are presented in the following section.

Table 6-1 illustrated functional performance and roughness parameters relation. A solid circle shows a significant amount of information linking the surface finish to the functional performance category, a half solid circle shows that there is some evidence and an open circle shows that there is some circumstantial indication [73].

\begin{tabular}{lcc}
\hline & Heights & Distribution and Shape \\
\cline { 2 - 3 } & Ra & Rsk \\
\hline Fatigue & 0 & 0 \\
Stress \& Fracture & 0 & 0 \\
\hline
\end{tabular}

- Much Evident $\quad \bigcirc=$ Some Evidence $\quad 0$ = Little or Circumstantial Evidence 
For surface roughness measurements, surface profilometry was used to examine the pre-fatigue machine surface. In order to establish a comparison between the two machining processes used, the surface roughness $R a$ was mainly considered as investigated from literature review. Figure 66 shows the micrographs of the machine specimens before being subjected to fatigue loading and after machining circular holes. It shows the effect of drilling processes on surface texture of the machined holes at different processing parameters as discussed in Section 3.2. All specimens after drilling holes showed no delamination. There was no material effect on the drilling process and final surface roughness parameters.

The hole wall surface features are captured in Table 6-2. Although the average surface roughness was similar for both types of specimens $(R a=3.50 \mu \mathrm{m}$ for AWJM and $R a=3.93 \mu \mathrm{m}$ for $\mathrm{CM})$ along the $\mathrm{x}$ and $\mathrm{y}$-axis, shown in Table 6-2, however the mechanical behaviour for these specimens was different as discussed above. These values showed that the comparatively lower surface roughness values for almost all the locations along the $\mathrm{x}$ and $\mathrm{y}$-axis were associated with specimens having a hole drilled with the AWJM process. To investigate the difference in the damage accumulation behaviour of both the specimens, the surface texture of the CM (Figure 66a) and AWJM (Figure 6-6b) specimens was examined. For specimens drilled with a conventional drill bit, the surface roughness photographs in Figure 6-6a show two discrete areas,

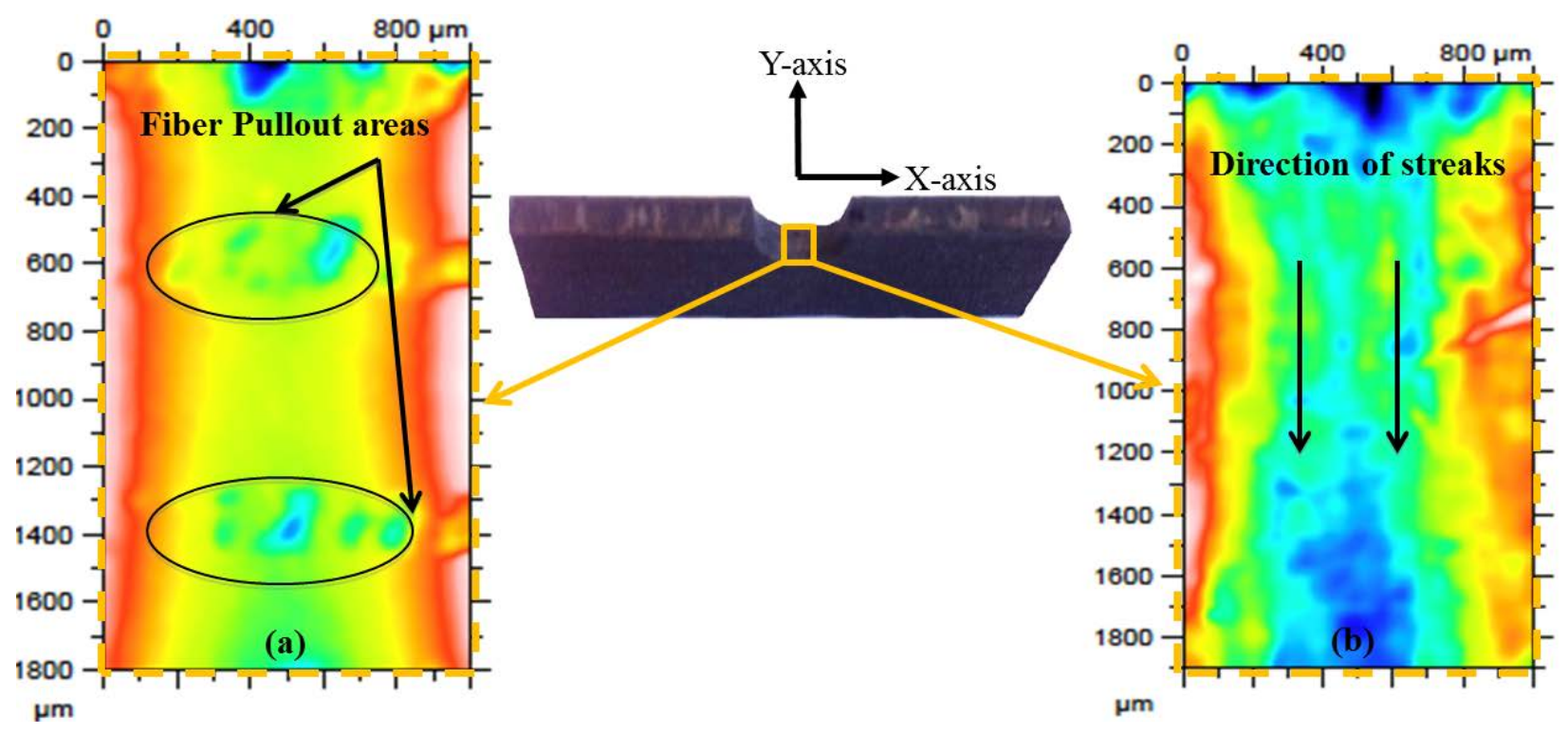

Figure 6-6 Cartography of the surface roughness of the wall of the hole for different specimens: (a) hole obtained with conventional machining using a cutting tool and (b) with abrasive waterjet. 
one with a rough surface finish which is shown as a protrusion while the second area has a smooth surface finish. The rough surface is one with the fiber pull-out and matrix degradation non-uniformly distributed on the wall of the drilled hole. These pull-out areas are related to the material removal mechanisms which are strongly influenced by the relative angle between the direction of the cutting speed and the direction of the fibers (Figure 6-6a). As during machining holes, a large amount of heat was also generated due to friction between workpiece and tool bit. The temperature reaches the glass temperature $\left(150^{\circ} \mathrm{C}\right)$ or even goes beyond, which may lead to localized heating and the softening of the matrix which causes the degradation of the composite laminate. Poor thermal conductivity and a difference in the coefficients of the thermal expansion of the fiber and the matrix may lead to localized heating, which was responsible for epoxy degradation (Figure 6-6a).

Table 6-2 2D Statistical roughness parameters of average surface roughness $R a$, maximum profile valley depth $R v$ and skewness Rsk for the two types of specimens, All values were obtained along the $\mathrm{X}$ and $\mathrm{Y}$ axis of the specimens

\begin{tabular}{cccc}
\hline Specimen & $\boldsymbol{R a}[\boldsymbol{\mu \mathrm { m } ]}$ & $\boldsymbol{R} \boldsymbol{v}[\boldsymbol{\mu \mathrm { m } ]}$ & $\boldsymbol{R s k}$ \\
\hline CM & $3.93 \pm 0.39$ & $15.44 \pm 2.53$ & $0.54 \pm 0.37$ \\
AWJ & $3.50 \pm 0.16$ & $12.85 \pm 1.30$ & $-0.20 \pm 0.10$ \\
\hline
\end{tabular}

In the case of the CM specimen, the maximum damage due to fiber pull-out was investigated when plies are oriented at $-45^{\circ}$ compared to the direction of the cutting speed. It showed that plies with $-45^{\circ}$ orientations were more prone to maximum damage when the conventional machining process was used. Fiber pull-out in the CM process owed to the inhomogeneous nature of composite material. However, the minimum damage is located in the zone where the fibers form an angle of $+45^{\circ}$ compared to the direction of the cutting speed. These observations are in good agreement with previous work conducted by Zitoune et al. [14] on the orthogonal cutting of UD composite specimens. Zitoune et al. [14] also observed that a continuous chip was formed by clear laminate shearing for $+45^{\circ}$ fibers. For the same fiber angle, a good surface finish was generated due to the perfect shearing of the fiber and matrix. The angle between the fiber 
orientations and the cutting speed direction influenced the mechanism of material removal and the mode of the rupture. Hence it was concluded that the machining process was influenced by the orientation of fibers under the action of the tool in the CM process.

The vibration of the machine and cutting tool also influenced the machine surface, which was not considered in the present study as an effort was made to eliminate vibrations during machining. To minimize the effect of vibration, the CNC machine and a collet chuck was used for the proper gripping of the specimen and drill bit.

By closely examining the AWJM specimen's surface morphology it was revealed that there were no distinct changes in the surface roughness of the AWJM specimen (Figure 6-6b) when compared with the conventional drilling process (Figure 6-6a). The AWJM specimens were characterized by streaks defect in the same direction of the displacement of the water-jet penetrated in the composite plate and craters defect due to the impact of the abrasive on the fibers. Globally striation marks and craters defects are uniformly distributed on the wall of the hole. No such prominent protrusion was observed as investigated in the specimens drilled with conventional tool (Figure 6-6a). The surface texture showed a uniform distribution of damage (streaks and crater defects) for the specimens machined with abrasive water-jet compared to the conventional drilled specimens. This surface texture was also seen and was confirmed by SEM (Figure 6-7) tests conducted after a destructive sectioning of the pre-fatigue testing specimens. SEM analysis confirmed the modes of degradation seen in the roughness analysis.

The results obtained from the NANOVEA 400 series showed that the maximum valley depth $R v$ was lesser for AWJM than CM specimens. $R v$ is indicated by the depth of the gap which is the distance between the center line and the lowest valley. The average $R v$ value for AWJM was 12.85 compared to 15.44 for the CM specimen, showing almost similarity. Another factor in addition to $R v$ was skewness (Rsk) whose average values were - 0.20 for the AWJM specimens and 0.54 for the CM specimens as shown in Table 6-2. Grzesik et al. have shown that negative Rsk values indicate surfaces with good bearing properties [145]. However in the current study, no prominent difference in the skewness value was observed.

From the research work stated above, it was observed that the surface roughness is commonly using for the characterization of the machined surface, however in present study, although the average surface roughness (cf. Table 6-2) was similar for both types of specimens, though the damage accumulation behaviour for specimens machined with different techniques was quite 
different. Hence, there is some disagreement in the literature and current study about the correlation between the workpiece surface roughness and fatigue life. In most cases in literature, it has been reported that lower roughness results in a longer fatigue life as explained earlier, while in the current study it was investigated that the surface texture damage was the main factor in determining the fatigue life rather than the average surface roughness $R a$ as the surface roughness in the current study was almost same for specimens drilled with two machining processes.
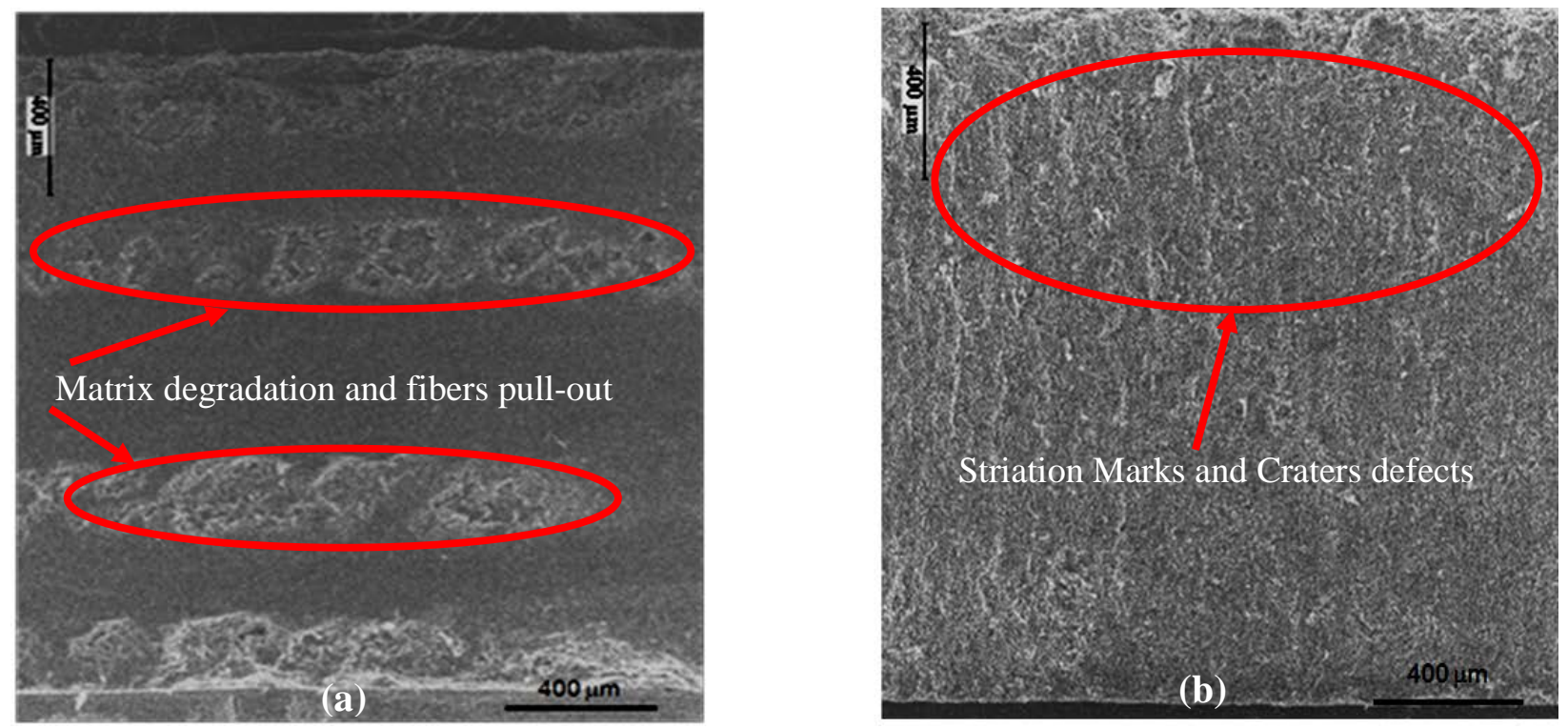

Figure 6-7 SEM photographs comparing the hole surfaces machined with two different techniques. A circular hole was machined with (a) conventional cutting tool and (b) Abrasive water-jet.

So in the current experiment, the characteristics of a CM specimen's surface, such as fiber pullout and matrix degradation have been shown in Figure 6-6a, influence fatigue failure more as shown in Figure 6-4 compared to the streaks and crater defects shown on the surface of the AWJM specimen (Figure 6-6b). Hence, the difference in the stiffness and damage accumulation between conventional and non-conventional drilled specimens was mainly attributed to the machining process. This was supported by the surface topography (roughness) tests as shown in Figure 6-6 which is already explained above. These damage areas (the fiber pull-out and matrix degradation area) on the conventional machined surface act as nucleation points for crack propagation which, with the application of cyclic load, caused a reduction of the mechanical 
performance of CM specimens compared to the AWJM specimen. One can conclude that unlike metallic materials, the criterion used for quantifying the quality of machining based on the average roughness $(R a)$ is not suitable for composite materials. Similar observations obtained from the surface roughness images on the hole's wall defects were confirmed by SEM micrographs as shown in Figure 6-7.

\subsubsection{Fatigue damage assessment of Angle ply and quasi isotropic lay-ups}

In order to investigate the fatigue damage accumulation in angle ply and quasi isotropic lay-up laminates drilled with the CM and AWJM processes, the initial and final stiffness of the specimens were first calculated from stress-strain data obtained from fatigue tests. Then, from Equation (1), the damage accumulation was calculated.

Figure 6-8 and Figure 6-9 show damage accumulation vs. the normalized cycles $\left(\mathrm{N} / \mathrm{N}_{\mathrm{f}}\right.$, where $\mathrm{N}_{\mathrm{f}}$ $=10000$ cycles), obtained for specimens with angle ply and quasi isotropic lay-up laminates drilled with AWJM and CM machining processes, respectively. From Figure 6-8, it was observed that the accumulated damage upto $5 \mathrm{kN}$ of the load was less than $10 \%$ for AWJM and around $10 \%$ for the CM specimens. Damage increased slowly to $10 \%$ at $6 \mathrm{kN}$ of a load for the AWJM specimen while at the same load, the damage increased moderately to $17 \%$ for $\mathrm{CM}$ specimens. Damage accumulation for the CM specimen increased rapidly to $32 \%$ at $7 \mathrm{kN}$ and the specimen rapture at $8 \mathrm{kN}$ load in less than 8000 cycles. However in the case of the AWJM specimen, at $7 \mathrm{kN}$ load, damage was less than $15 \%$. The damage increased rapidly to $25 \%$ for an
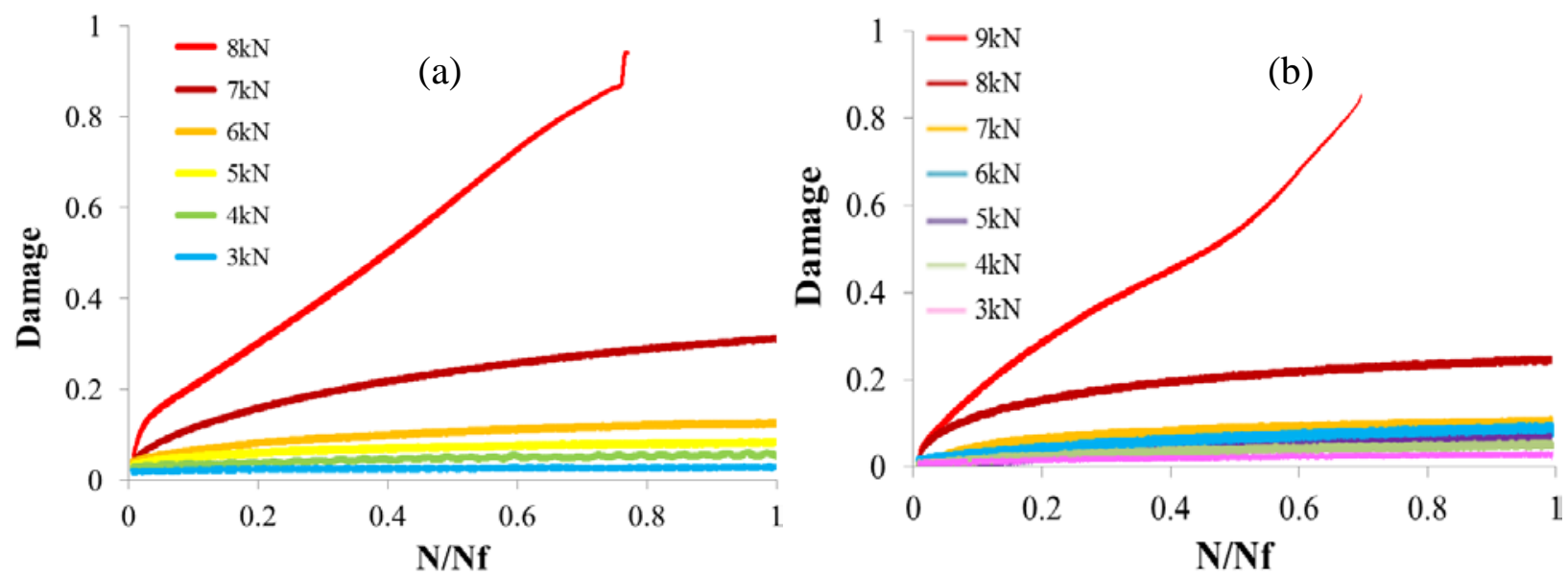

Figure 6-8 The evolution of damage for various level of for CF/Epoxy quasi isotropic specimens machined with (a) CM and (b) AWJM technique, tested at $10 \mathrm{~Hz}$ frequency and 10000 cycles. 
$8 \mathrm{kN}$ load and the AWJM specimen rapture at $9 \mathrm{kN}$ load. The CM specimens tested for 10000 cycles in each loading step failed earlier than the specimens tested at 5000 cycles (Section 6.2.1). It shows that increasing the processing parameters reduce the fatigue life, however, the difference of the machining process was still a factor affecting the mechanical behaviour. It was worth mention here that for $\left[ \pm 45^{\circ}\right]_{2 S}$ specimens, loaded for $10 \mathrm{~Hz}$ frequency and 10000 cycles, the damage was gradually and continuously increasing in each loading step as compared to the stabilization in damage which was due to the saturation in damage at 5000 cycles as previously investigated in Section 6.2.1, that mainly owed to the fatigue testing parameters. This continuous increase in damage in each loading step reduced the life of the specimen. However, in both studies, the influence of the machining effect can be seen from the early failure of the CM compared to the AWJM specimens.
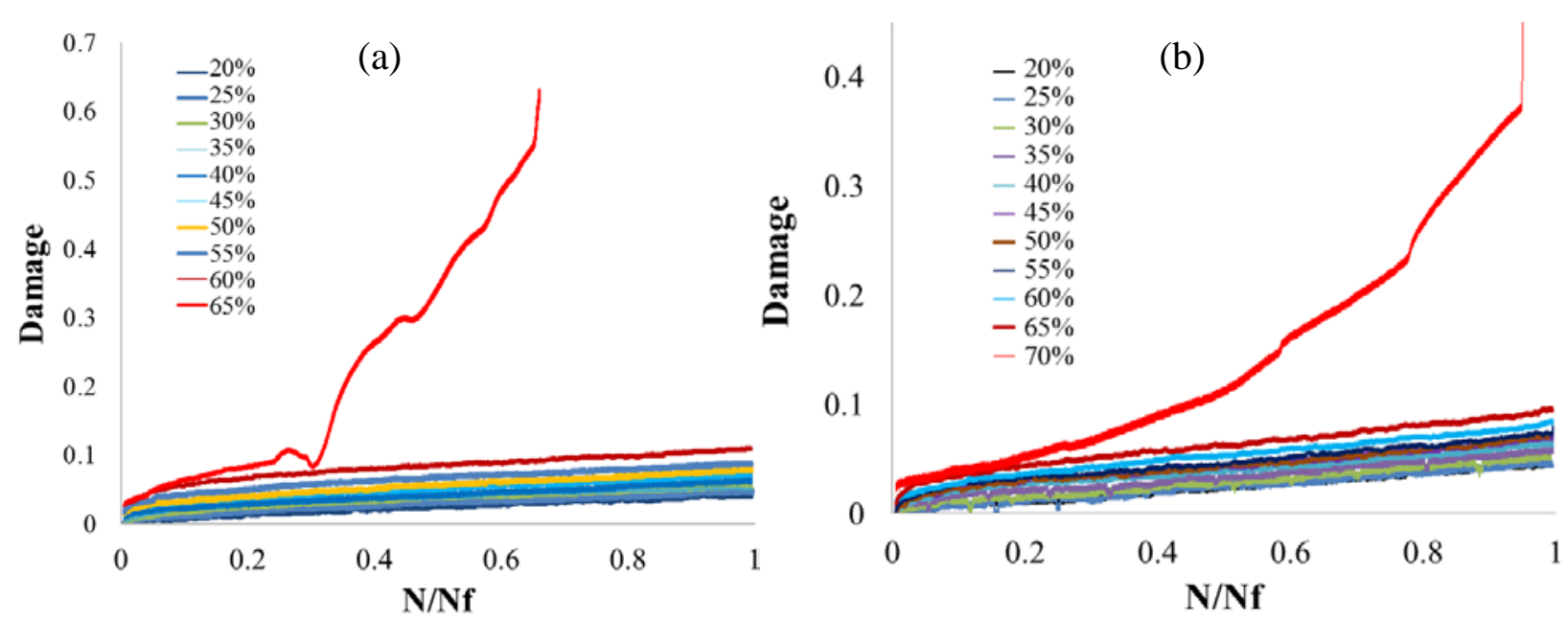

Figure 6-9 The evolution of damage for various level of loading for CF/Epoxy quasi isotropic specimens machined with (a) CM (b) AWJM technique, tested at $10 \mathrm{~Hz}$ frequency and 10000 cycles

In the case of quasi isotropic CM laminates (Figure 6-9), the accumulated damage was around $10 \%$ at a load of $60 \%(20.82 \mathrm{kN})$ of UTS, while in the case of AWJM, it was less than $10 \%$ at the same load. The damage increased rapidly at a load of $65 \%$ of UTS for the CM specimen and finally the specimen failed at around a 65\% load of UTS. However, for the AWJM specimen, the damage increased moderately to $10 \%$ at a $65 \%$ load of UTS then, the damage increased suddenly and the AWJM specimen failed at a 70\% load of UTS consuming almost 10000 cycles of the same loading stage. 
It was also investigated that the quasi isotropic specimens failed in a more brittle manner and showed linear mechanical behaviour, whereas angle ply CF/Epoxy specimens showed progressive damage due to a non-linear mechanical response. Since the damage increased almost uniformly till the second last loading step for all the quasi isotropic specimens and then suddenly failed in brittle manner, this behaviour was mainly attributed to the fiber dominated $\left(0^{\circ}\right)$ plies.

The reason for the difference in the damage accumulation of the CM and AWJM specimens, shown in Figure 6-8 and Figure 6-9 was investigated from the surface roughness images presented in Figure 6-10 and Figure 6-11 for angle ply and quasi isotropic specimens, respectively. Figure 6-10a and Figure 6-11a show fiber pull-out and matrix degradation areas on the surface of the CM specimens whereas Figure 6-10b and Figure 6-11b illustrated the streak marks on the surface created by the abrasive water-jet machining process. These damages induced by machining processes were mainly responsible for the difference in the fatigue life of the two type of laminates used for this study as already discussed in Section 6.2.1, although surface roughness $R a$ was the same for all tested laminates.
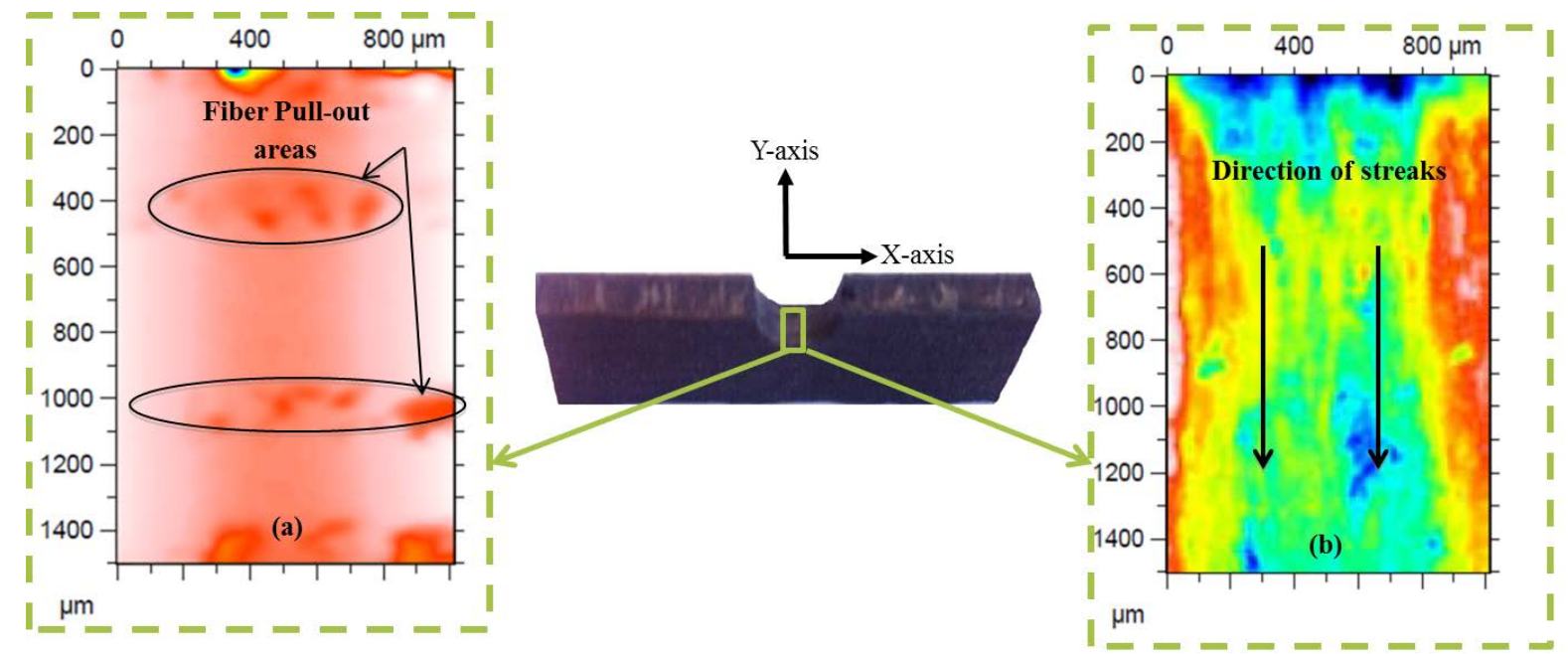

Figure 6-10 Surface Roughness images showing the cross section of circular hole of CF/Epoxy angle ply lay-up laminates, pre-fatigue test, a circular hole obtained with the (a) CM (b) AWJM techniques.

\subsubsection{Crack Density}

The pre-fatigue machined surface was analyzed with image analysis software for the percentage area of cracks produced by the machining processes. Image analysis step-by-step procedure is shown in Figure 6-12. The percentage area of cracks was calculated from ImageJ Software from 


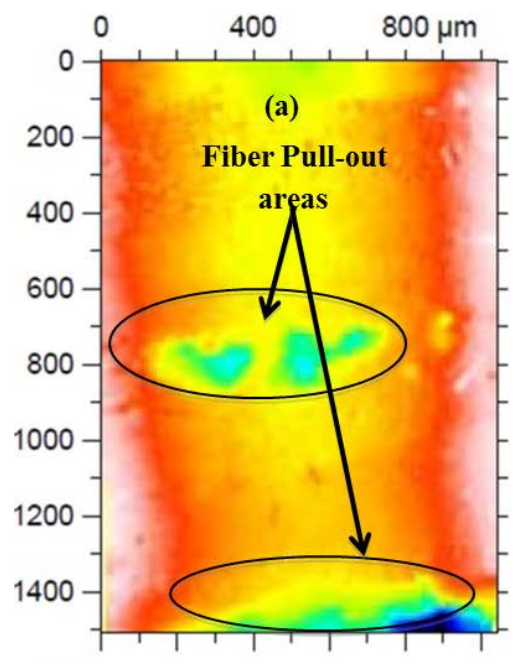

$\mu \mathrm{m}$
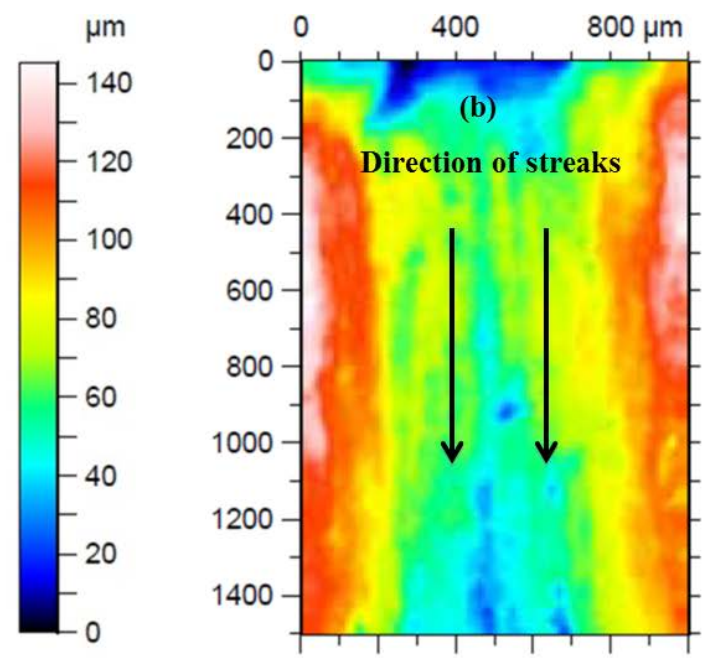

$\mu \mathrm{m}$

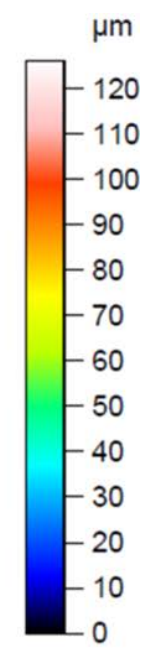

Figure 6-11 Surface Roughness images showing the cross section of circular hole of quasi isotropic lay-up laminate, pre-fatigue test, a circular hole obtained with the (a) CM and (b) AWJM techniques.

Table 6-3 Surface roughness of pre-fatigue CF/Epoxy assembly specimens drilled with the CM and AWJM processes.

\begin{tabular}{lcccc}
\hline & \multicolumn{4}{c}{ Surface roughness $(\mathbf{R a}) \boldsymbol{\mu m}$} \\
\cline { 2 - 5 } \multicolumn{1}{c}{ Description } & $\begin{array}{c}\text { Stacking sequence of angle ply } \\
\text { lay-up laminate }\end{array}$ & $\begin{array}{c}\text { Stacking sequence of quasi } \\
\text { isotropic laminate }\end{array}$ \\
\cline { 2 - 5 } & $\begin{array}{c}\text { Surface roughness } \\
(R a) \mu \mathrm{m}\end{array}$ & $\begin{array}{c}\text { Surface } \\
\text { roughness } \\
(S a) \mu \mathrm{m}\end{array}$ & $\begin{array}{c}\text { Surface } \\
\text { roughness } \\
(\mathrm{Ra}) \mu \mathrm{m}\end{array}$ & $\begin{array}{c}\text { Surface } \\
\text { roughness }(\mathrm{Sa})\end{array}$ \\
\hline $\begin{array}{l}\text { Conventional machined } \\
\text { specimens }\end{array}$ & $4.06 \pm 0.34$ & 12.03 & $4.54 \pm 0.51$ & 14.60 \\
$\begin{array}{l}\text { Abrasive water-jet } \\
\text { machined specimens }\end{array}$ & $3.59 \pm 0.26$ & 14.20 & $4.16 \pm 0.17$ & 14.60 \\
\hline
\end{tabular}

the National Institute of Health and are presented in Table 6-4. A higher crack density in the CM speciemns was due to the machining process used for drilling holes, consequently resulting in a higher damage accumulation. On the other hand, the AWJM specimens having a low crack density and streak defects on the machined surface consequently resulted in less damage accumulation. 

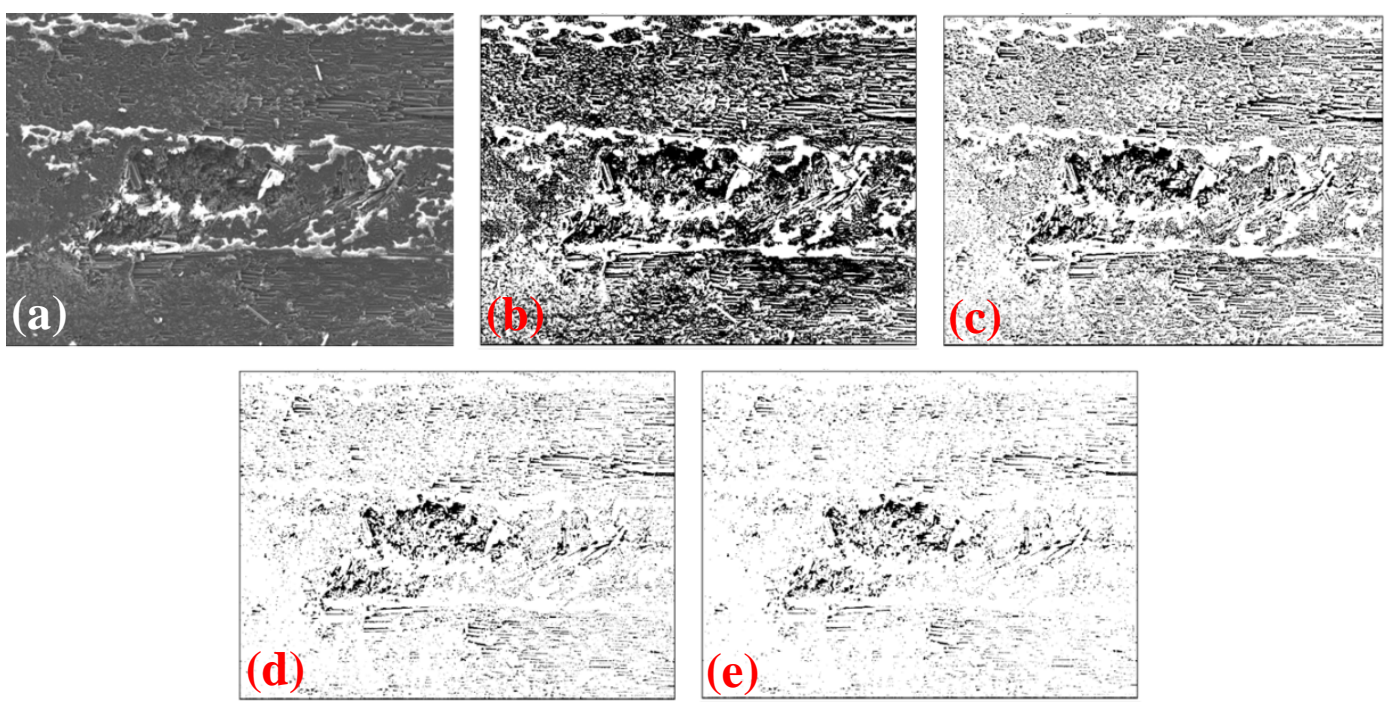

Figure 6-12 Image processing step by step procedure to determine the damage region.

Table 6-4 The \% area of crack of angle ply and quasi isotropic CF/Epoxy laminates machined with (a) CM and (b) AWJM technique, pre-fatigue testing

$\%$ area of crack (Crack density)

Description
Angle ply lay-up Quasi isotropic lay-up
laminate
laminate

Conventional Machined specimens

$8.77 \pm 2.14$

$5.39 \pm 1.71$

Abrasive water-jet machined specimens

$1.68 \pm 1.96$

$0.62 \pm 0.48$

\subsection{Thermal Analysis for Angle ply Laminate}

Heat dissipation during fatigue testing was studied for different specimens drilled with two machining processes. The infrared thermography technique was used in fatigue tests to obtain various images showing the examined specimen's surface temperature distributions during each cyclic step loading stage.

Two types of specimen were used for fatigue tests. During the tests, increasing loads from 3 to $11 \mathrm{kN}$ (18 to $63 \%$ of UTS) with the increments of $1 \mathrm{kN}$ in each step for a period of 5000 cycles, were applied. The change in temperature $(\Delta T)$ was recorded by the IR camera for each specimen 
for all the loading stages shown as curves in Figure 6-13 and Figure 6-14 (Appendix A-8 and Appendix A-9 for all tested specimens). These curves show load stages after $3 \mathrm{kN}$ of load. These temperature curves can be explained with infrared thermographic images shown in Figure 6-15 and Figure 6-16. Figure 6-15 showed the surface temperature at 3, 8 and $10 \mathrm{kN}$ (these loads represent respectively $18 \%, 47 \%$ and $59 \%$ of UTS) for the CM and the surface temperature contours recorded by the IR camera for AWJM specimen under load levels of 3, 8 and $11 \mathrm{kN}$ (these loads represent respectively 18\%, 47\% and 65\% of UTS) is shown in Figure 6-16 and there curves are presented in Figure 6-13 and Figure 6-14. All the images represented in these figures are obtained at 5000 cycles. The images recorded by the IR camera show that, for loads less than $7 \mathrm{kN}$, the temperature remains constant throughout the surface in the vicinity of the hole as shown in temperature maps ( Figure 6-15a and Figure 6-16a) and both specimens have shown similar damage and temperature increase. For a load of $8 \mathrm{kN}$, temperature fluctuations increase but remain moderate (Figure 6-15b and Figure 6-16b). For loads above this value, the temperature and damage was more important for the specimens drilled using CM process. However, for the loads at $10 \mathrm{kN}$ for the $\mathrm{CM}$ and at $11 \mathrm{kN}$ for the $\mathrm{AWJM}$ specimens, there were

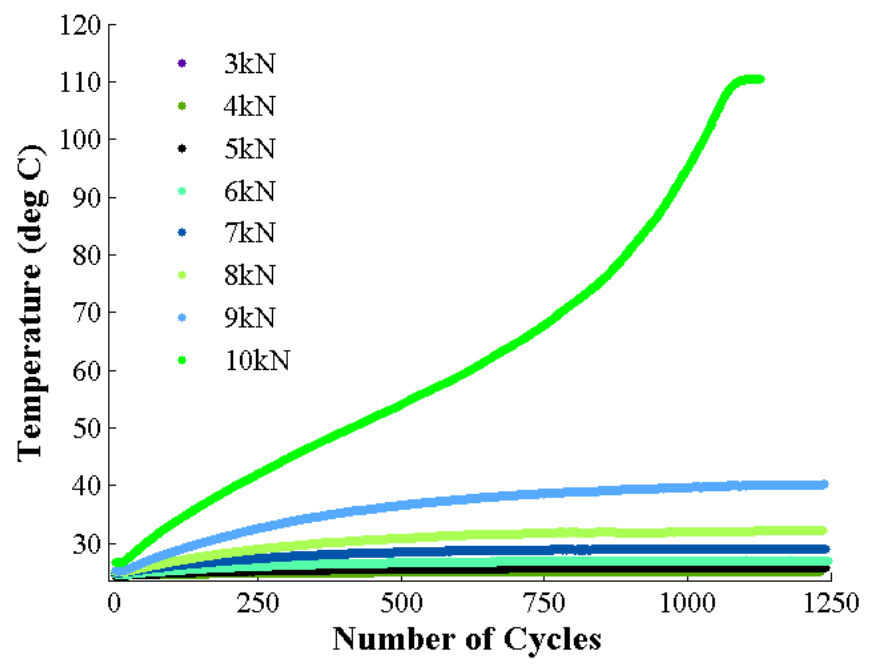

Figure 6-13 Stages of the temperature changes for fatigue loading. In each stage, a load was applied for 5000 cycles $(1250 \times 4)$ till rupture for the CM specimen. A similar temperature trend was also obtained for all the CM specimens

changes in the thermo-mechanical behaviour of the material and a significant increase in temperature and the fluctuations in the study area were observed which reached to $100 \%$ as shown in Figure 6-15 and Figure 6-16 respectively. In the tests conducted at $10 \mathrm{kN}$ (59\% of 
UTS) shown in Figure 6-15c the CM specimens ruptured around mean cycles of $4140\left(\mathrm{~N} / \mathrm{N}_{\mathrm{f}}=\right.$ 0.8 ) at a temperature of $110^{\circ} \mathrm{C}$. Under the same loading, after the same number of cycles, the AWJM specimens continued to withstand cyclic stresses and the temperature rise was around $50^{\circ} \mathrm{C}$, a difference of more than $100 \%$ was recorded when compared to the CM specimen. The AWJM specimens lasted all 5000 cycles at the same cyclic load without rupturing, dissipating a maximum temperature value of only $50^{\circ} \mathrm{C}$. These results indicated that for any given load level, AWJM specimens dissipated less temperature than CM specimens. The AWJM specimens ruptured after $1181 \pm 341$ mean cycles corresponding to a temperature increase up to a value of $78.5 \pm 7^{\circ} \mathrm{C}$ at $11 \mathrm{kN}$ load level. This showed that the CM specimens generated more heat and failed earlier at a $10 \mathrm{kN}$ load level corresponding to the failure at $11 \mathrm{kN}$ load level for all of the AWJM specimens with less heat dissipation. These values showed that the temperature of the specimens rise significantly at these loads and the slope of the temperature component suddenly moves to a higher value in the CM specimens than the AWJM specimen which was because of fiber pull-out and matrix degradation locations generated in the CM process which dissipated more heat and damage than the AWJM specimens having streaks and crater defects on the machined surface (cf. Figure 6-6). The higher temperature of the CM specimens produced higher localized plastic deformation which was responsible for the early fatigue failure of the specimen. This area was situated at $\pm 45^{\circ}$ from the axis of the load and also represents the axis of the

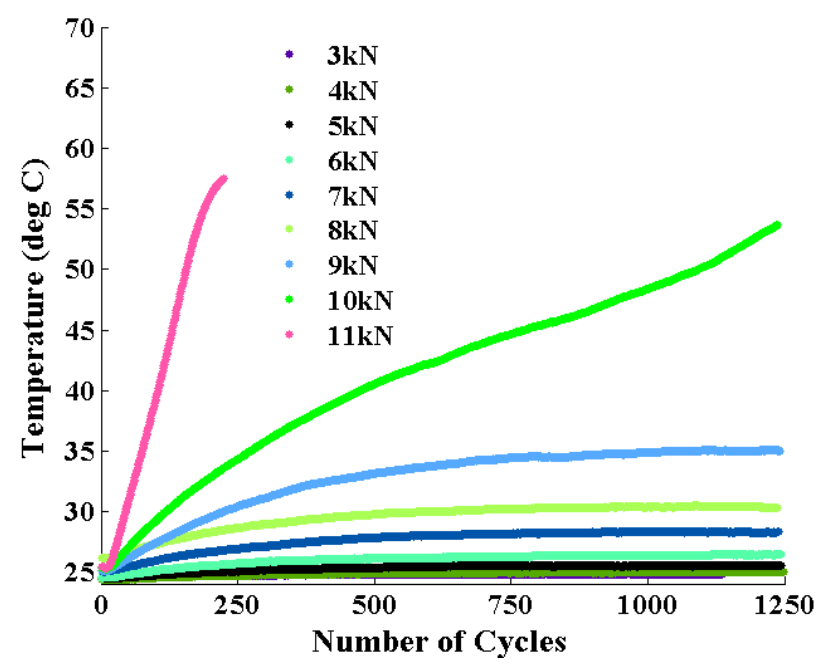

Figure 6-14 Stages of the temperature changes for fatigue loading. In each stage a load was applied for 5000 cycles (1250 x 4) till rupture for the AWJM specimen. A similar temperature trend was also obtained for all AWJM specimens. 


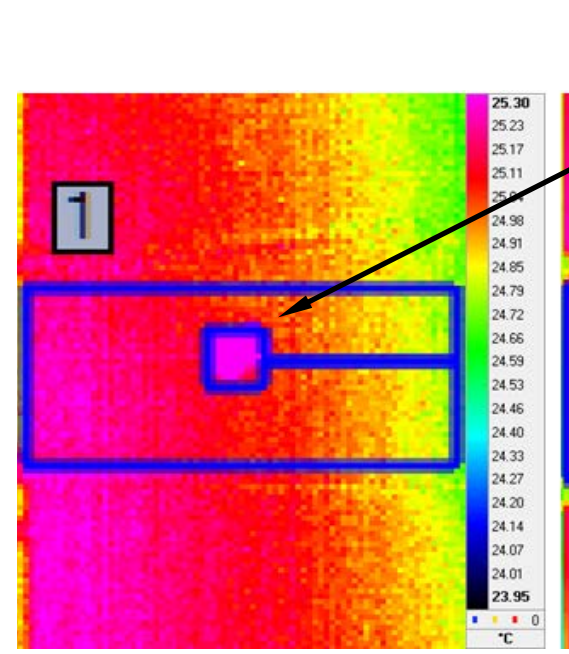

(a)
Areas considered for analysis

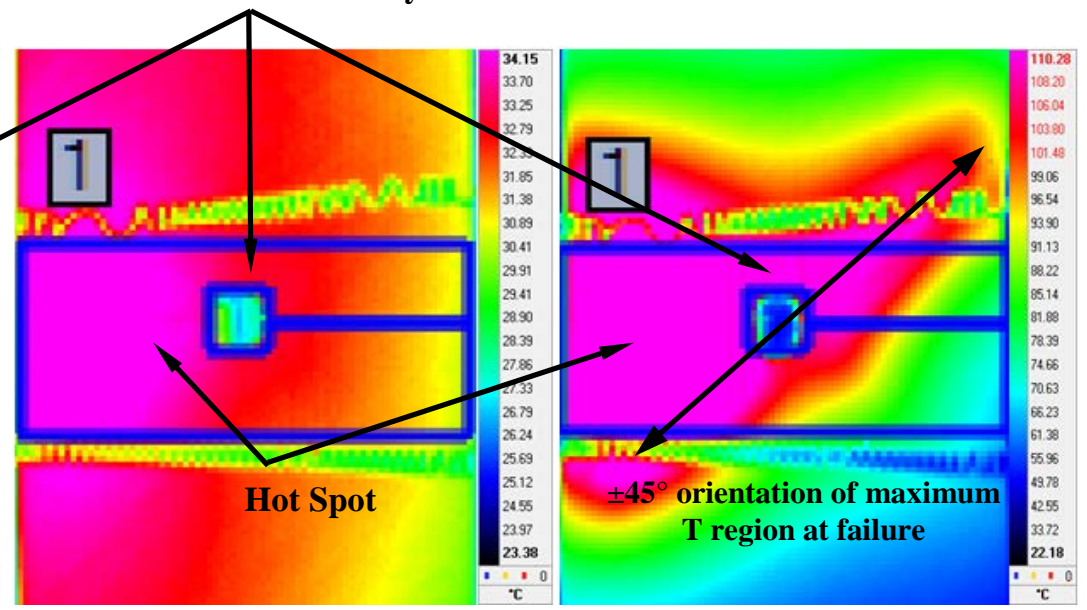

(b)

(c)

Figure 6-15 Temperature contours for the CM specimens at 5000 cycles under (a) $3 \mathrm{kN}$, (b) 8 $\mathrm{kN}$ and (c) $10 \mathrm{kN}$ cyclic loads. The area around the hole within the rectangle is considered for investigation.

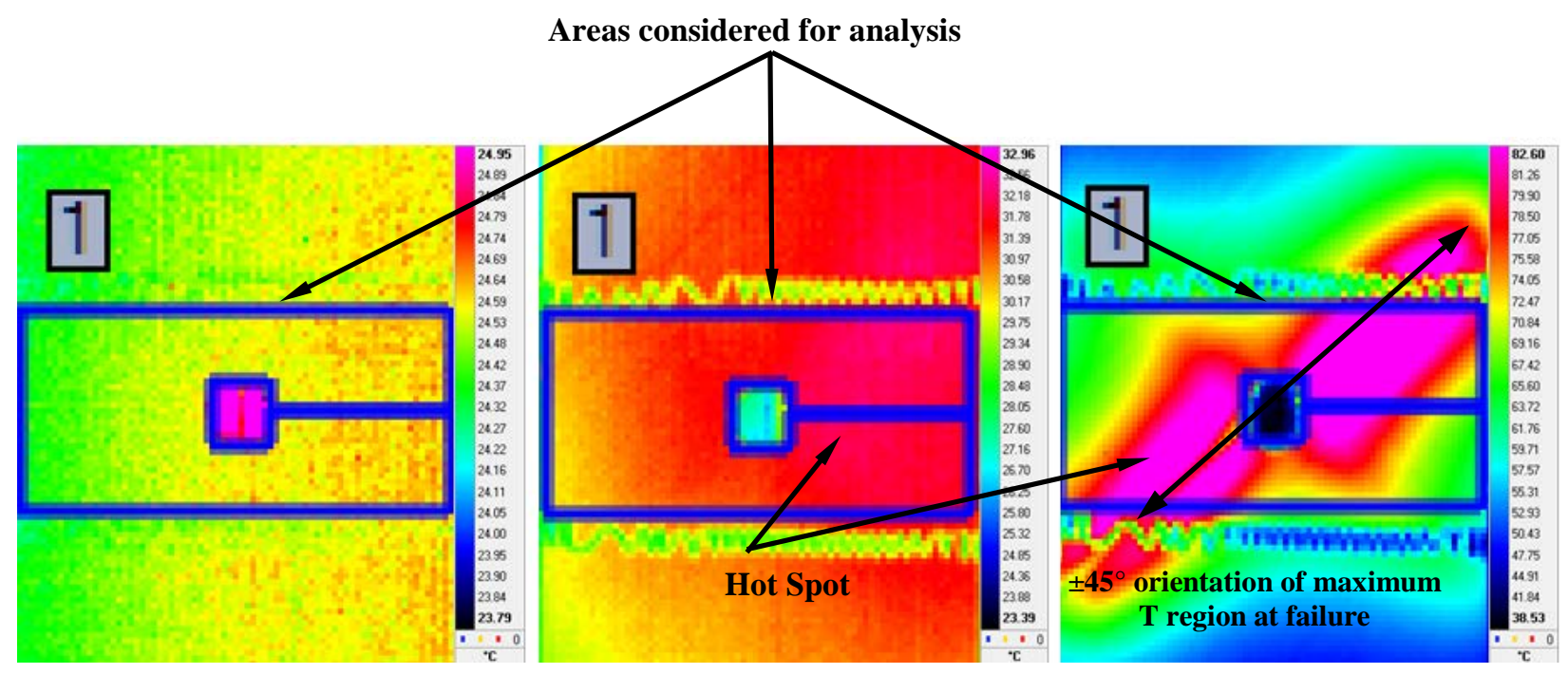

(a)

(b)

(c)

Figure 6-16 Temperature contours for AWJM specimens at 5000 cycles under (a) $3 \mathrm{kN}$, (b) 8 kN and (c) $11 \mathrm{kN}$ cyclic loads. The highest temperatures are in pink/violet and the lowest are in black/dark blue. The area around the hole within the rectangle is considered for investigation.

direction of the plies and the direction of the failure. A similar pattern was also observed in the case of specimens drilled with non-conventional machining. However, at higher loads, the temperature fluctuations in the CM specimens were more significant than those machined with the abrasive water-jet process for the same loading. The excessive and sudden heat generated in 
the CM specimens was recorded at the stress concentration areas which were fiber pull-out and matrix degradation areas that created more damage and heat dissipation than the striations marks observed on the surface of AWJM specimens. Except the machined surface which is shown as a hot spot (Figure 6-15 and Figure 6-16), no other damage location was found in infrared thermography images. These images represented the increase in the maximum surface temperature as a function of the fatigue life for a tension-tension fatigue test.

In literature [146, 147] increased in temperature was related to the matrix cracking, delamination and fiber breakage. The evolution of the temperature appears according to three stages (Figure 613 and Figure 6-14). In the first stage, the variation in the temperature was due to the friction of the fibers/matrix and to the damages which started and were propagated during the fatigue test. In the second stage, the temperature reached a balance that was due to saturation in the damage and crack propagated in the matrix/fiber interface (Figure 6-3). Due to the reversible thermoelastic heating and cooling during each initial loading cycle, a small temperature variation was observed. As there was a small quantity of heat loss, the temperature remains at a constant value during most of the initial fatigue life. The high sensitivity and thermal noise improvement provided by the IR camera gives the final temperature resolution of the instrument to be less than $1 \mathrm{mK}$ which helps in detecting the small mean temperature rise induced by energy dissipation inside the specimen during the early stages of loading in both specimens. The stability observed in the second stage was followed by an abrupt increase of the temperature in stage three. High temperature showed high localized deformation which was responsible for the rupture of the composite plates as the deformation of solid materials are almost always accompanied by release of heat. When the material becomes deformed or is damaged and fissured, a part of the energy necessary to starting and propagating the damage is transformed in an irreversible way into heat [26].

Finally, it was determined that IR thermography is a useful inspection technique for flat surfaces, and is used to identify sub-surface flaws represented in the form of hotspots in IR images as shown in Figure 6-15 and Figure 6-16. 


\subsection{Damage assessment of angle ply and quasi isotropic laminates using IR thermographic}

Figure 6-17 and Figure 6-18 illustrated temperature evolution of angle ply lay-up laminates drilled with CM and AWJM processes whereas in the case of quasi isotropic laminates, infrared thermographic images shown in Figure 6-19 and Figure 6-20 depicted the temperature evolution for the specimens drilled with CM and AWJM processes.

Figure 6-17 showed that the temperature almost remained same till 5kN of load for angle ply CM specimens. Further increase of loading until $7 \mathrm{kN}$, the temperature increased moderately (Figure 6-18c). Similarly for the AWJM specimen (Figure 6-18a), initially the temperature was comparable to the $\mathrm{CM}$ and remained uniform until a $5 \mathrm{kN}$ load. The temperature increased moderately $\left(28^{\circ} \mathrm{C}\right)$ with the increase in load till $7 \mathrm{kN}$. This temperature in the case of the CM specimens was achieved at a $5 \mathrm{kN}$ load. A further increase in the load resulted in a rapid raise of temperature for both specimens, consequently the CM and AWJM specimens raptured with the

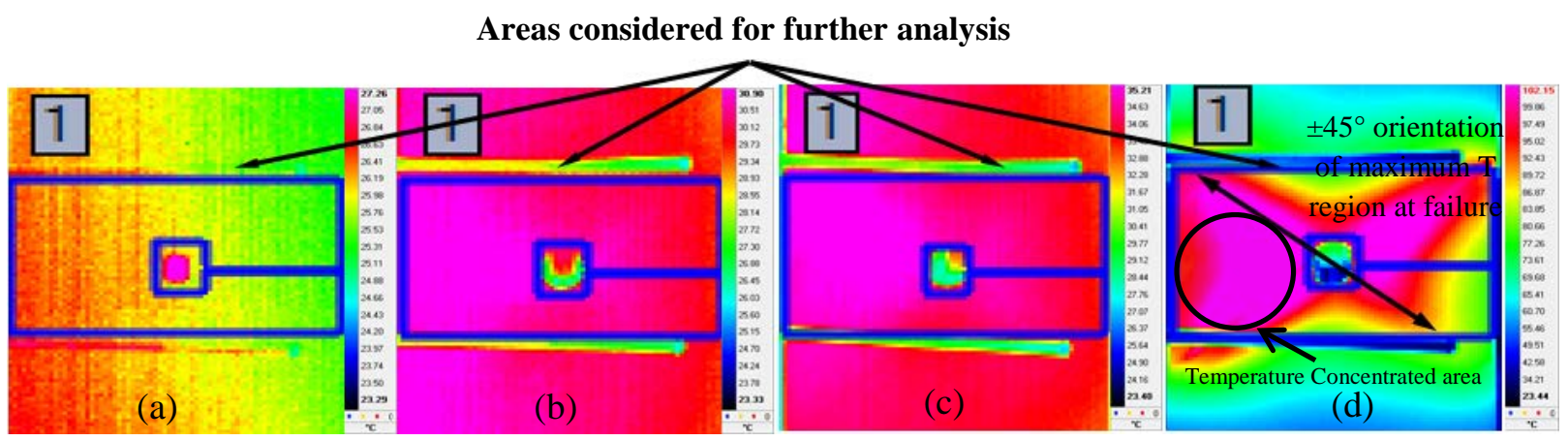

Figure 6-17 Temperature maps for loads (a) $3 \mathrm{kN}$, (b) $5 \mathrm{kN}$ (c) $7 \mathrm{kN}$ and (d) $8 \mathrm{kN}$ of angle ply CM specimen at $10 \mathrm{~Hz}$ frequency and 10000 cycles.

sudden increased in temperature, at 8 and $9 \mathrm{kN}$ of load, respectively. However the failure temperature of the CM specimens was more than the AWJM specimens, which was around $102^{\circ} \mathrm{C}$ and $74^{\circ} \mathrm{C}$ respectively. This high temperature for the case of the $\mathrm{CM}$ specimens also showed more damage accumulation, as shown in Figure 6-9. Furthermore, Figure 6-18 showed more temperature concentration in $\pm 45^{\circ}$ direction, which was also the failure direction of angle ply lay-up laminates. The temperature concentration was more in the $-45^{\circ}$ direction for the CM specimens as has been shown in Figure 6-17 (b, c, d) than in the AWJM specimens (Figure 618). This can easily understood from Figure 6-10a, which showed fiber pull-out and matrix 
degradation on the surface of the $\mathrm{CM}$ specimens, which was responsible for more heat dissipation and damage. A similar observation was also noted for the CM and AWJM for the case of quasi isotropic laminates, where temperature increased exponentially when the loading cycle increased. However, the failure of quasi isotropic laminates occurred at a lesser temperature than angle ply lay-up laminates, i.e. $48^{\circ} \mathrm{C}$ compared to $102^{\circ} \mathrm{C}$ for $\mathrm{CM}$ and for the case of AWJM specimens it was $39^{\circ} \mathrm{C}$ compared to $74^{\circ} \mathrm{C}$, respectively.

Figure 6-19 (images c and d) depicting that temperature concentration started in a $-45^{\circ}$ and $90^{\circ}$

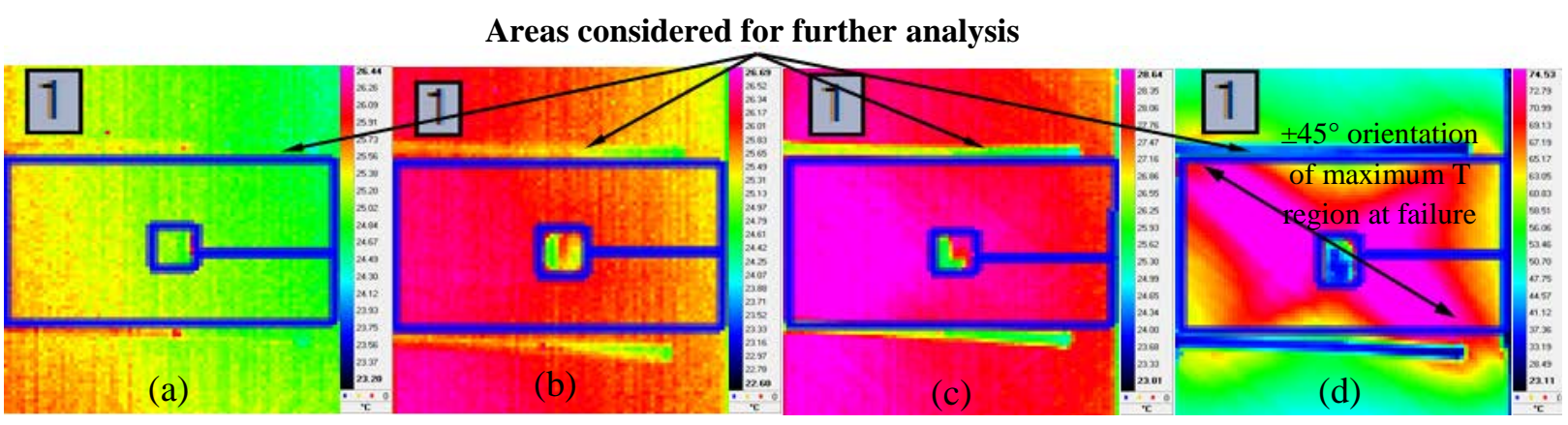

Figure 6-18 Temperature maps for loads (a) $3 \mathrm{kN}$, (b) $5 \mathrm{kN}$ (c) $7 \mathrm{kN}$ and (d) $9 \mathrm{kN}$ of angle ply AWJM specimen at $10 \mathrm{~Hz}$ frequency and 10000 cycles.

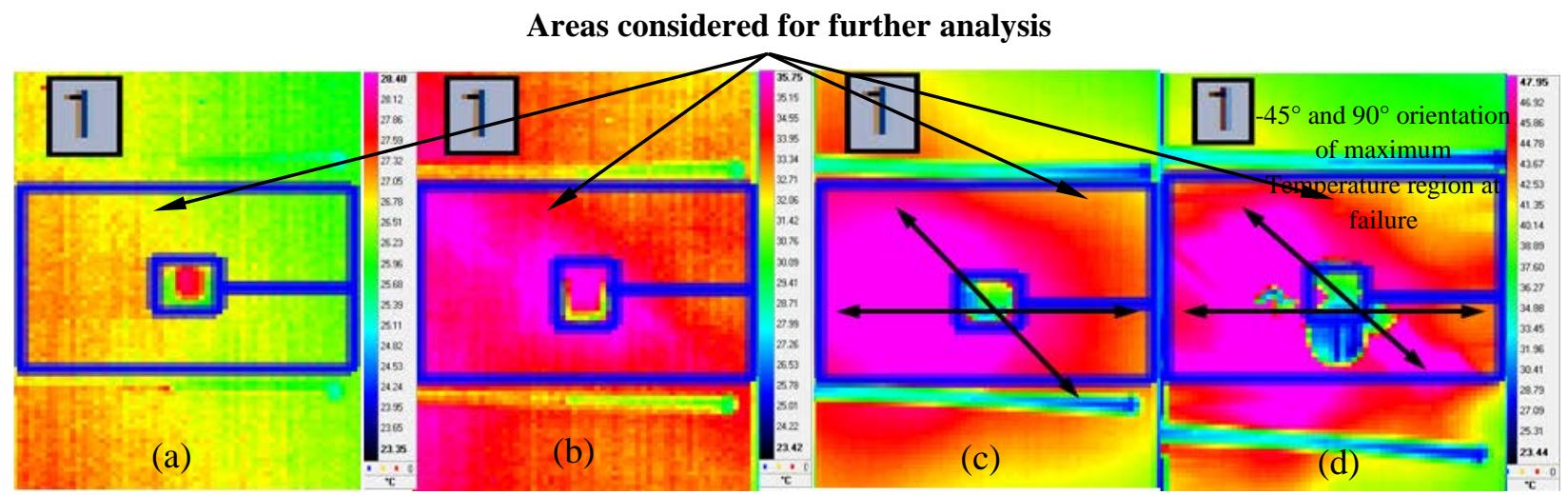

Figure 6-19 Temperature maps for loads (a) 20\%, (b) 55\% and (c) and (d) $65 \%$ of quasi isotropic CM specimen at $10 \mathrm{~Hz}$ frequency and 10000 cycles.

direction as these plies were more effected by CM process as shown in surface roughness images of quasi isotropic laminates (Figure 6-11a). However, in the case of quasi isotropic laminates drilled with AWJM process (Figure 6-20), these plies were least affected by the drilling process 
used. In this case, the failure first initiated and propagrated in $90^{\circ}$ plies which were matrix dominated plies and were more affected by the increase in applied cyclic load. Failure initiated and propagrated in a $90^{\circ}$ direction was evident from the temperature concentration in this direction (Figure 6-20d). Generally, in this case (AWJM), the increase in temperature and damage was related to matrix degradation, fiber pull-out/breakage and delamination during fatigue cyclic loading. Initially, the increase in temperature was related to the degradation of the matrix at weak/damage portion of the specimen. Consequently, fiber/matrix delamination and debonding takes place, which resulted in a further increase in temperature and damage as the number of loading cycles increased. A further increase in the loading cycles caused an abrupt increase in temperature, resulting from the breaking of the fibers. This increase in temperature continued till the final failure of the specimen [146, 147].

From the above discussion, it can also be observed that damage accumulation and temperature can be related with each other. Therefore, at this stage of the current investigation, we will try to obtain a correlation between the thermographic analysis and the damage accumulation which is presented in the following sections.

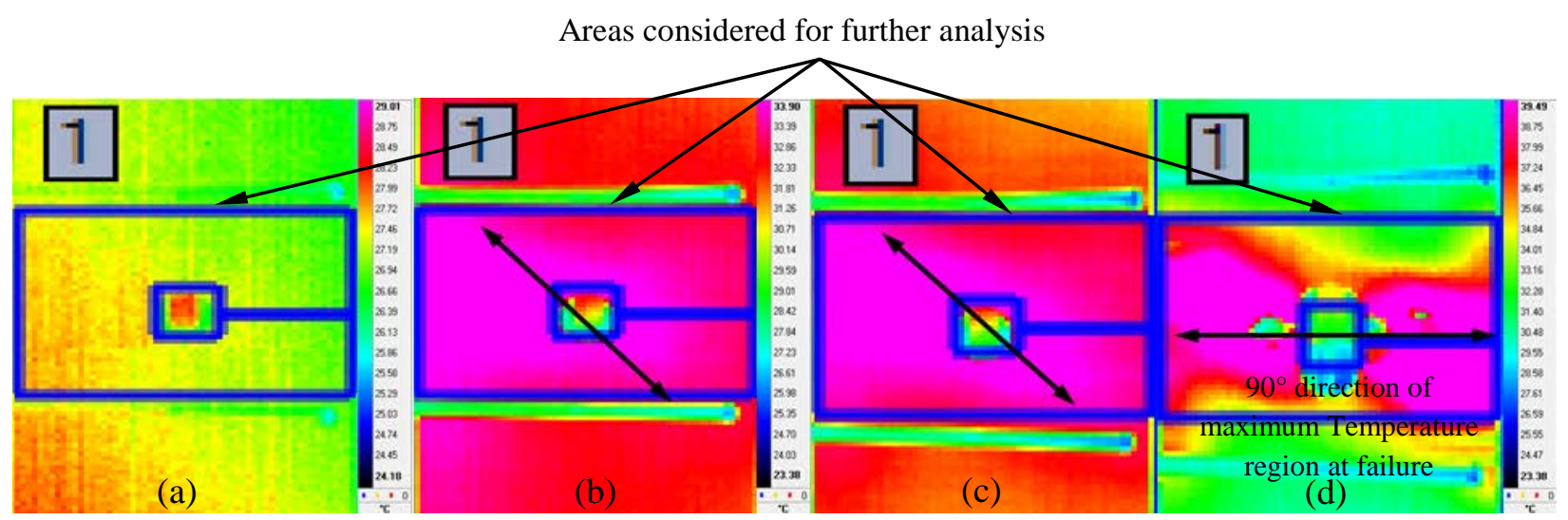

Figure 6-20 Temperature maps for loads (a) 20\%, (b) 55\% and (c) and (d) 70\% of quasi isotropic AWJM specimen at $10 \mathrm{~Hz}$ frequency and 10000 cycles.

\subsection{Correlation between Heat Dissipation and Damage accumulation}

Up till now, the damage accumulation curves and temperature maps were analyzed in Sections 6.2 to 6.4. Now the surface temperature raised measured from the heat dissipation is correlated with the damage accumulation, determined by the IR non-destructive testing (NDT) technique to evaluate two drilling processes. From the above discussion (Sections 6.4), it was 
observed that the damage growth due to fatigue loading stimulates a significant increase in temperature. As it is known that the deformation of a structure is usually followed by heat dissipation. When the material is deformed or is damaged, a part of the energy necessary to the starting and the propagation of the damage is irreversibly transformed into heat $[26,123,148]$. Therefore in the current study it is necessary to measure this heat loss in order to correlate it with the damage of the specimen.

Figure 6-21 depicts the evolution of the maximum temperature and damage before the final failure of the specimen against normalised number of cycles. The CM and AWJM specimens showed almost uniformity and the temperature change was less than $1^{\circ} \mathrm{C}$ for load stages of $6 \mathrm{kN}$ and $8 \mathrm{kN}$. This heat dissipation is a form of energy, which is responsible for damage accumulation. So fatigue damage is closely related to plastic deformation and heat dissipation, which affects the temperature of the materials. It was observed that there was a strong similarity between the trends of the damage assessment curves and those representing the change in temperature and a strong, direct correlation between the recorded temperature and the damage accumulated around the hole. The temperature of the studied area (region within the rectangle in Figure 6-15 and Figure 6-16) rises as the damage accumulates. The direction of the damage propagation was according to the applied load in the same way as the temperature. This trend corroborates the observations of Toubal et al. [26].

The evolution of the maximum temperature and damage accumulation before the final failure of the specimen occurs according to three stages as depicted in Figure 6-21. In first stage, the variation of the temperature is due to the thermo-elasticity of the material and the friction between layers (i.e. fibers/fibers and/or fibers/matrix), whereas, in second stage, the temperature reaches a balance that is due to saturation in the damage. With the increase in load, the rate of the damage and the friction become more important. This stability is followed by an abrupt increase of the damage and temperature of the specimen corresponding to the thermo-mechanical effects, plasticity and failure [26, 114].

The thermal dissipation and damage accumulation results discussed in this section indicate that the defects produced on the wall of the hole in the CM holes (fiber pull-out and matrix degradation, Figure 6-6a and Figure 6-7a) tend to stimulate more damage under long-term cyclic loading than the defects in the AWJM holes (striations on the wall of drilled hole, Figure 6-6b and Figure 6-7b). Based on the above analysis, we can say that the type of defects generated by 
machining processes as shown in Figure 6-6 may be the cause of this variation in the thermal dissipation and damage accumulation of the composite laminates. At this stage, the following question may arise:

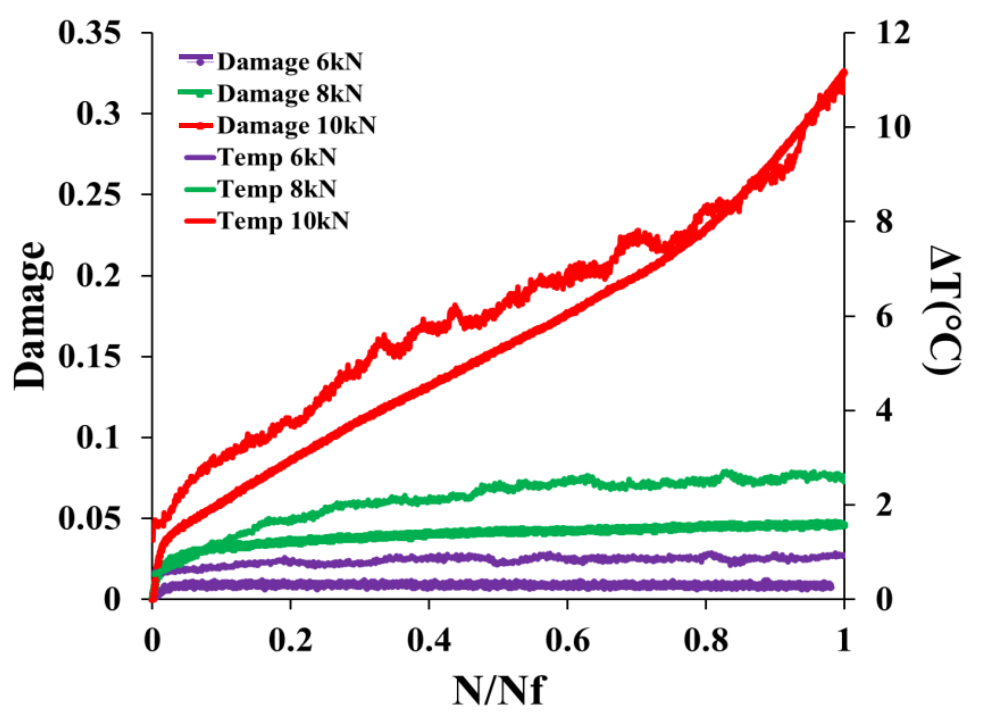

Figure 6-21 Comparison between the change of the maximum temperature and the damage for AWJM specimen at a different level of loading: $6 \mathrm{kN}, 8 \mathrm{kN}$ and $10 \mathrm{kN}$.

Do the machining-related defects observed in Figure 6-6 and Figure 6-7 influence the limit of endurance? To answer this question, further analysis is indeed needed.

\subsection{Endurance Limit}

An engineering material can support a stress level without failure when cyclic loading is applied to it. Below this safe load the material can withstand an infinity number of cycle without failure. This safe load is called the fatigue endurance limit [122].

In general, the endurance limit is obtained by Whöler curves (stress vs cycles). So the endurance limit in such case can be obtained from the temperature stabilization $(\Delta \mathrm{T})$ curves $[122,123]$ by intersecting the two straight lines that interpolate the stabilization temperature and the corresponding stress level as shown in Figure 6-22. The endurance limit can be found as the intercept of the curve on the $x$-axis. Thus the endurance limit can be defined as the stress level at which an evident change in the stabilized temperature $(\Delta \mathrm{T})$ take place as shown in Figure 6-23. This can be obtained from the following equation; 


$$
\Delta \mathrm{T}=\mathrm{T}_{\mathrm{f}}-\mathrm{T}_{0}
$$

where $\Delta \mathrm{T}$ is the change in temperature, $\mathrm{T}_{\mathrm{f}}$ is the final temperature and $\mathrm{T}_{0}$ is the initial temperature.

Using the above concept on the current research's experimental data, we can obtain a correlation between the thermographic analysis and stress to obtain the endurance limit as presented below.

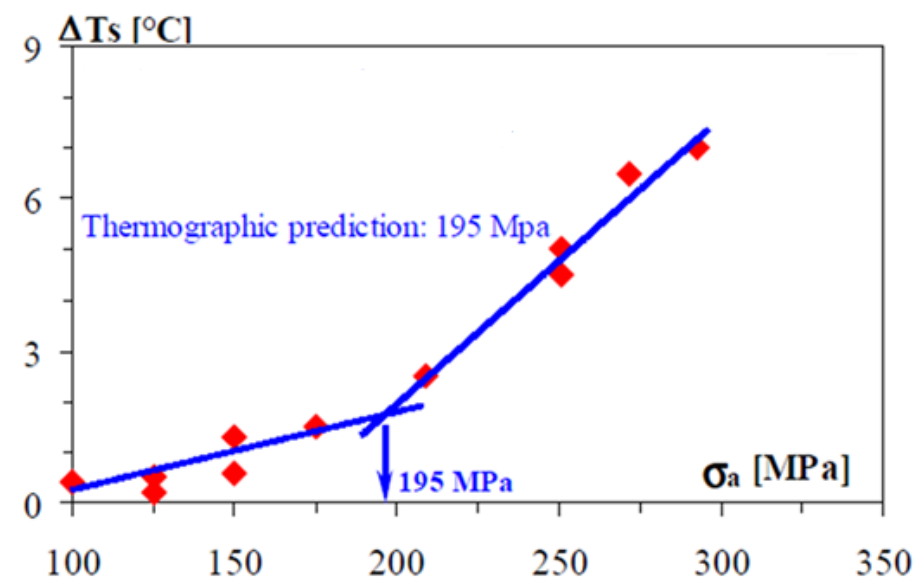

Figure 6-22 Endurance limit prediction [122]

\subsubsection{Results and Discussion}

IR experiments were performed on ten specimens drilled with two different machining processes. From the temperature $(\Delta \mathrm{T})$ and stress experimental data, various plots were obtained, where the change in temperature $(\Delta \mathrm{T})$ was observed with respect to the corresponding stress. The temperature gradient at the beginning of the loading cycle and the stabilized temperature profile at 5000 cycles for different loads for the CM and AWJM specimens that were shown in the graphs between temperature profile $\Delta \mathrm{T}$ and stress, in Figure 6-23. After the application of the load when the temperature of the specimen is stabilized, the endurance limit can be observed as described above (Section 6.6). The temperature increase was considerable when the loading level exceeds the endurance limit. As can be seen, the endurance limit of the specimen drilled using CM (Figure 6-23a) is lower than that of the AWJM (Figure 6-23b). There was virtually no significant change in heat dissipation was observed when the stress amplitude was lower than 93.5 $\pm 0.76 \mathrm{MPa}$ and 83.3 $\pm 4 \mathrm{MPa}$ for five conventionally drilled specimens and five water-jet specimens, respectively. So initially the values of the mean rise in temperature $(\Delta T)$ can be 
considered as negligible for all specimens, irrespective of the drilling process used as all curves show linearity in data points. The slope of the first curve shows that the stress amplitude changes largely with less increase in temperature. Initially a less increase in temperature corresponded to the less damage accumulation. It was also observed that around 93.5 $\pm 0.76 \mathrm{MPa}$ for AWJM and 83.3 $\pm 4 \mathrm{MPa}$ for the CM specimens some changes in the thermo-mechanical behaviour occurred and the stresses at this point are higher than the elastic limit and the thermoelastic law is no longer valid and micro-plasticity started to appear. The average endurance limit for AWJM specimens was $10 \%$ more than specimens drilled with CM process, however all the specimen show an almost similar trend. Progressive increase in temperature of the specimens during each loading step was observed when the specimens was loaded above the endurance limit. Therefore, a considerable increase in the slope of second curve was observed. Thus, after the endurance limit, the temperature $(\Delta \mathrm{T})$ rises more rapidly with less change in stress so failure occurred with less increase in stress but at high temperature.
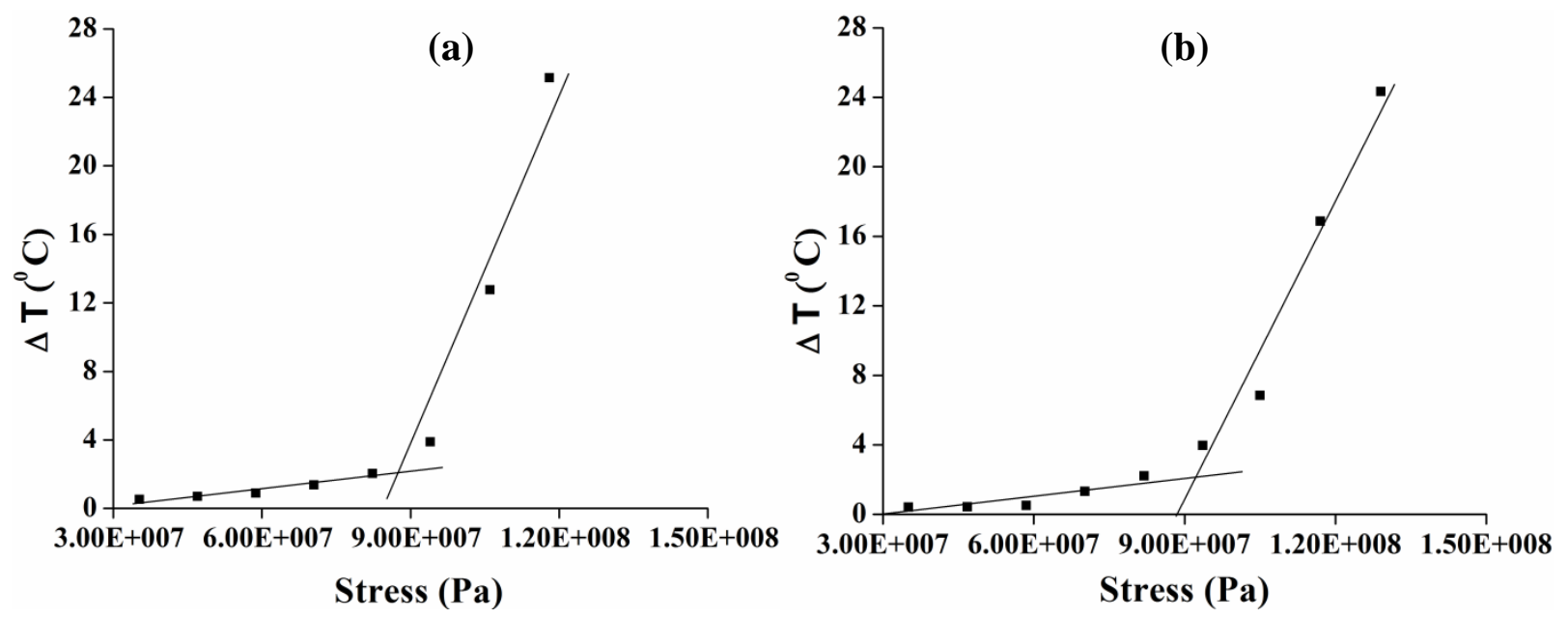

Figure 6-23 Graphical representation of endurance limits for the specimens (a) CM and (b) AWJM. The endurance limit obtained by intersecting the two straight lines that interpolate the experimental data of the stress and temperature.

The temperature curves obtained from Equation (45) when specimens were tested at 10,000 cycles for different step loading stages and for the specimens drilled with CM and AWJM processes having angle ply and quasi isotropic lay-up are presented in Figure 6-24. 
The endurance limit of angle ply laminate (Figure 6-24) was $90.01 \pm 0.045$ and $78.14 \pm 1.53 \mathrm{MPa}$ for AWJM and CM specimens, respectively. It showed that AWJM overestimated the CM specimens by $13.19 \%$. However, when comparing with the specimens tested at 5000 cycles, the
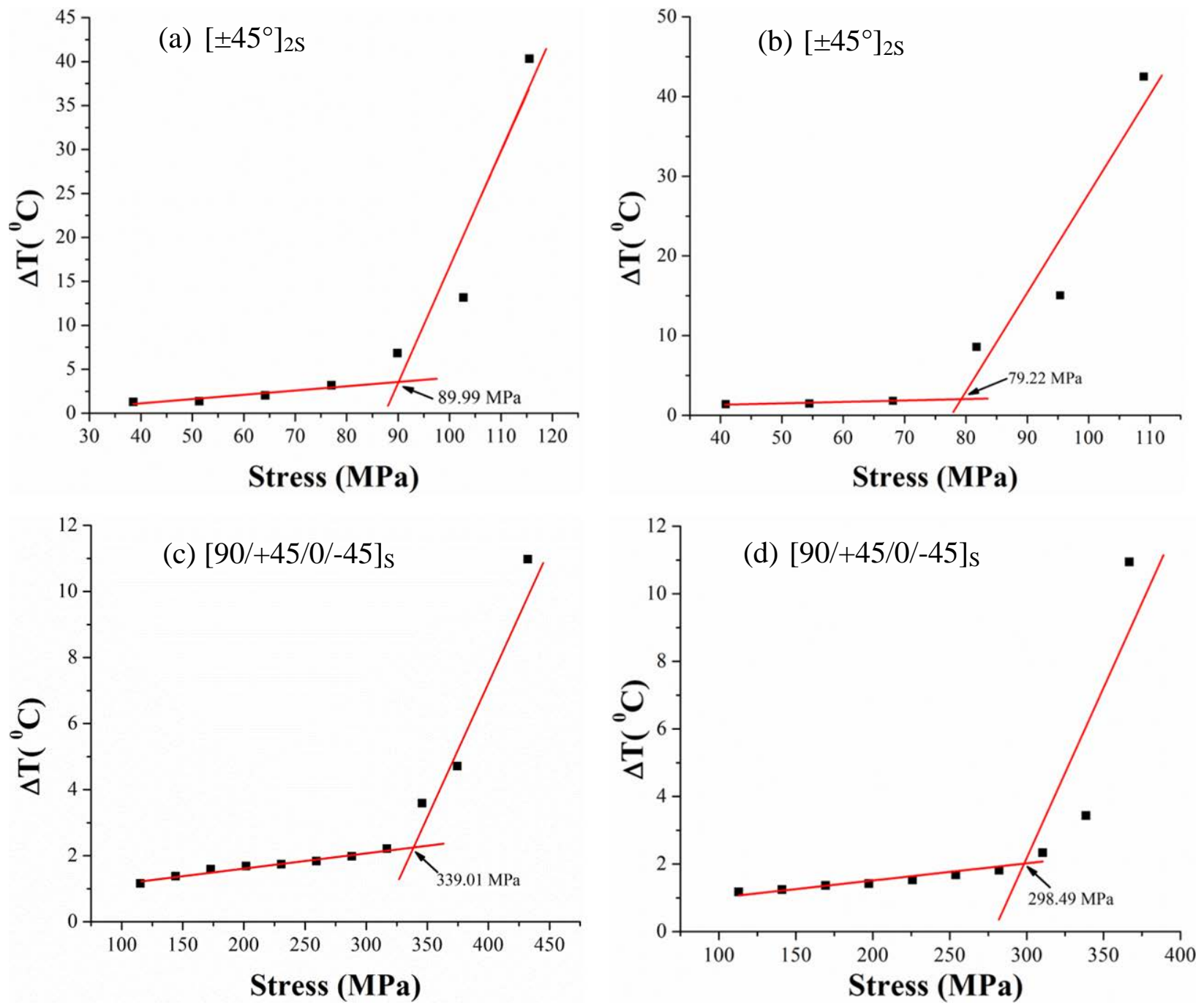

Figure 6-24 The endurance limit of CF/Epoxy specimens machined with (a)(c) AWJM and (b)(d) CM processes, tested at $10 \mathrm{~Hz}$ frequency and at 10000 cycles.

difference in endurance was less than 10\%. Similarly for quasi isotropic lay-up laminate, endurance limit was 298.05 \pm 2.72 and 339.02 $\pm 0.007 \mathrm{MPa}$, respectively for the CM and AWJM specimens which showed an improvement of 12.08\% in AWJM over CM specimen. A lower endurance limit of both laminates drilled with the CM process was owed to the fiber pull-out and degraded matrix as observed on the machined surface presented in Figure 6-10 and Figure 6-11. 
Similar results were obtained during the fatigue tests of pin loading specimens (compressioncompression) conducted by Persson E et al. [11] on specimens drilled by two processes of machining. The authors have obtained that, specimens drilled with an unused Polycrystalline Diamond (PCD) drill (conventional machining) present a failure strength of around 19\% lower compared to the specimens drilled with the KTH method (orbital drilling) using a diamond graincoated tool. Similarly Taylo D. et al. [144] found that machined surfaces with different surface roughness values, generated by the conventional machining process, has an obvious influence on endurance limit of the specimens, consequently, authors found that surface roughness has strong influence on endurance limit.

Based on the above analysis, we can advance that the type of defects generated by machining processes as shown in Figure 6-10 and Figure 6-11 may be the cause of this variation in the endurance limit values of the composite laminates. The endurance limit decreased for CM specimens as there was fiber pull-out and damage area in the matrix which created more crack initiation sites than AWJM specimens having striation marks. As the endurance limit decreased by 10-13 percent, the crack initiation occurs more rapidly in the vicinity of hole (in the fiber pullout and matrix damage areas) in the CM than in AWJM specimens. These results clearly showed that the signature of the machining process has an important role on the mechanical behaviour of a machined composite structure part.

The size of inclusions plays an important role in fatigue life, it acts as a stress concentration area. When its magnitude is higher than the $R a$ value then it is responsible for $50 \%$ reduction in endurance limit [149]. In the present study, the number of voids was negligible so the fatigue life was only dependent upon the surface texture already explained in Section 6.2.1.

\subsection{Thermographic damage criterion}

The study conducted by Wei et al. [148] proposed a criterion quantifying the rate of damage based on the measurement of the damage area recorded by infrared thermography. In this case, the damage rate was calculated by;

$$
D\left(N_{i}\right)=\frac{A\left(N_{i}\right)}{A\left(N_{f}\right)}
$$

where $D$ is the damage function, $A\left(N_{i}\right)$ is the damage area measured at the $i^{\text {th }}$ cycle and $A\left(N_{f}\right)$ is the damage area at the number of cycles to failure. The results presented by the authors showed a 
correlation between the damage predicted by this method (Equation 46) and the one measured by the conventional method (loss of rigidity). However, no threshold chosen to quantify the evolution of the damage inside the studied zone was given (Figure 6-25). In the present study, extrapolating from the work of Wei et al. [148], we propose to quantify the damage rate with a new approach, by determining a critical threshold (Figure 6-26). As the damage accumulation and maximum temperature rise was more concentrated towards the end of each loading stage at 5000 cycles, as shown in Figure 6-15, Figure 6-16 and Figure 6-26. So there was a need to explore these areas for damage accumulations as increased in temperature represented the damage accumulation. So these populated area was considered for the damage evolution in current study. For damage and analysis of heat dissipation, circular hole was eliminated (Figure 6-27) as this open area added the ambient temperature to the specimen's temperature which could affect the minimum and maximum temperature of the specimen. Thus this research proposes damage comparison and fatigue failure prediction using a thermal threshold - the

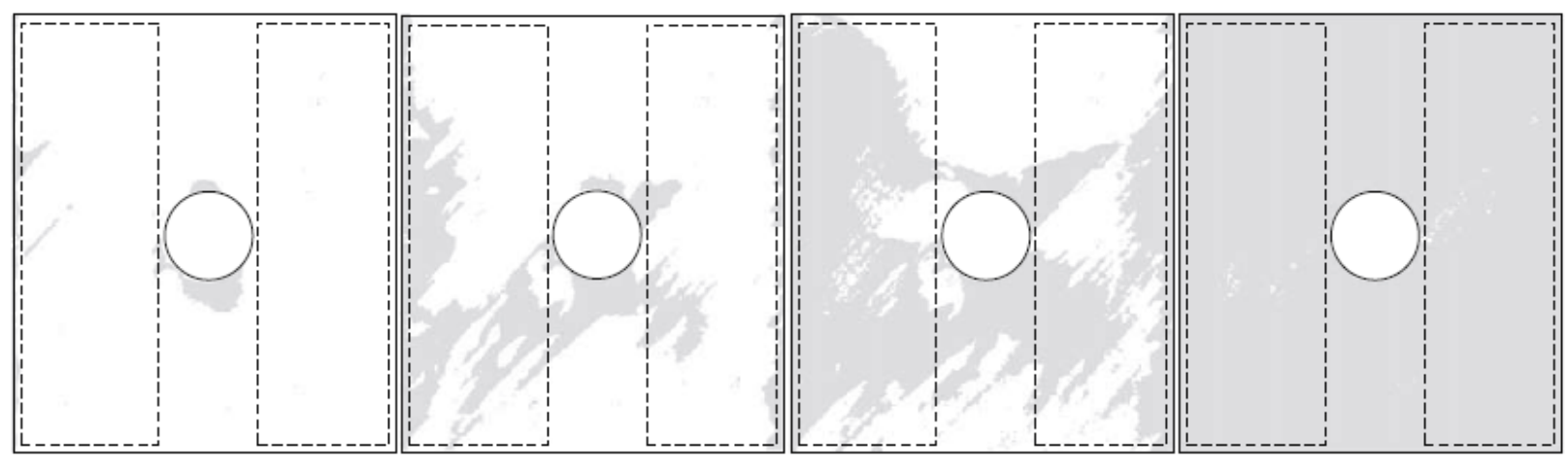

Figure 6-25 Cumulative damage zone under increasing fatigue cycles [148]

Thermographic Damage Criterion (TDC) to observe the behaviour of the damage accumulation in both machining processes. Thermographic damage criterion (TDC) is an area ratio, based on the area of maximum temperature recorded in infrared thermographic images (Figure 6-15, Figure 6-16 and Figure 6-26). The TDC was observed at different fatigue loading stages for each specimen. For this purpose following criterion was developed and was used;

$$
T D C=\frac{A_{i}\left(\Delta T_{\max }\right)}{A_{\text {total }}}
$$

where TDC is IR thermographic damage criterion, $A_{i}\left(\Delta T_{\max }\right)$ is the area (pixels) of the maximum 
Thermal threshold area

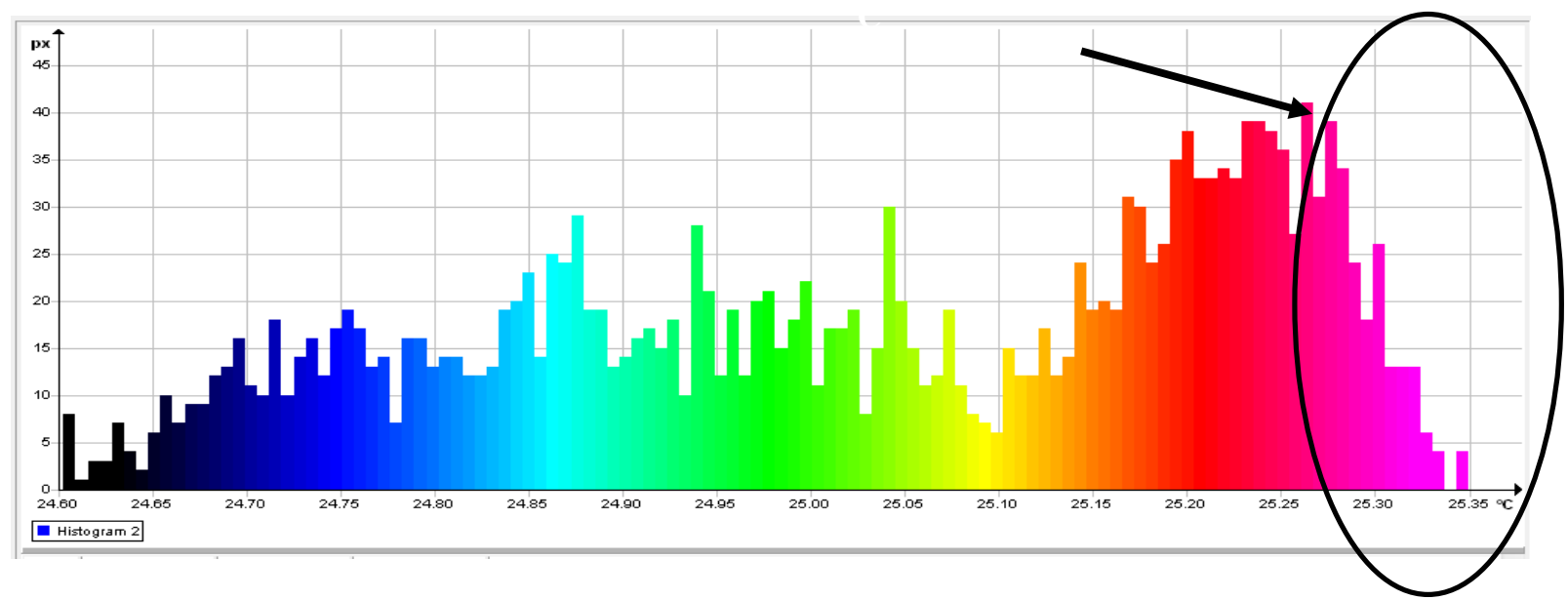

Figure 6-26 shows the thermal threshold area considered in Equation (47) for evaluating the damage

temperature region at the $i^{\text {th }}$ cycle (as shown in Figure 6-26) and $A_{\text {total }}$ is the area of the overall sample region under study as shown in Figure 6-27. For all analysis initial condition of 3kN load was considered as reference point to examine the temperature and damage accumulation for each loading conditions at 5000 cycles. By using Equation (47) we can plot the TDC over each fatigue test for the two specimens made of AWJM and one specimen made of CM (cf. Figure 6-28).

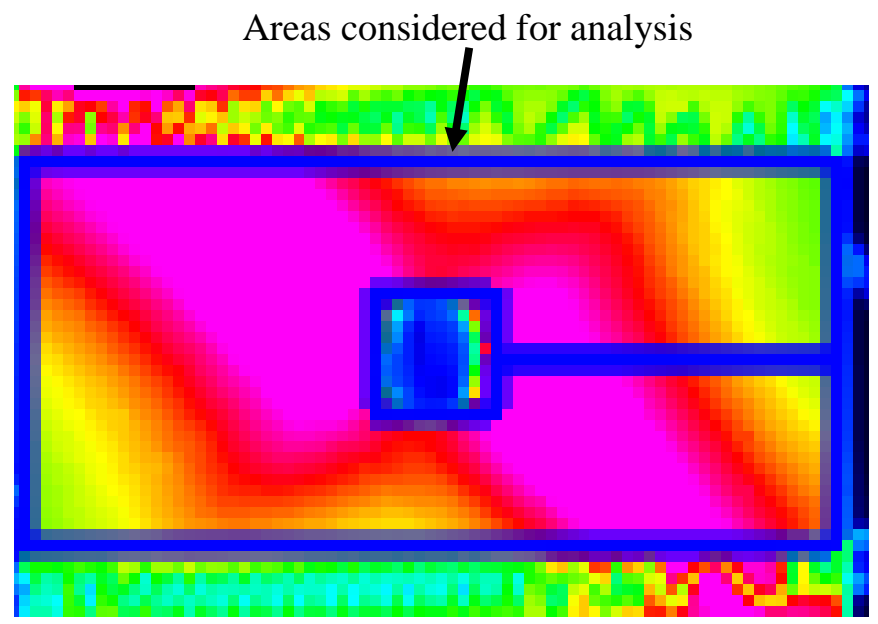

Figure 6-27 Infrared thermographic image showing thermal threshold area

Figure 6-28 showed that in early stages of fatigue life, the change in temperature was less, consequently the damage area was also less for all specimens and moved uniformly till the $8 \mathrm{kN}$ load for CM and AWJM specimens. After these load stages, the thermal threshold area increased 
as the fatigue cycle increased in the CM specimen. It also showed that for a given loading stage, the thermal threshold area was the least in the case of AWJM specimens than in the CM specimens as the number of cycles and load increased till $10 \mathrm{kN}$ load. The same behaviour can also be seen from Figure 6-29, where temperature changes for both types of specimens were the same up to $8 \mathrm{kN}$ load. After that it increased more rapidly in the CM specimens and it was continuous till failure. Thus, it was observed that, for all applied loads, the TDC of specimens machined with AWJM was lower than the TDC of specimens drilled with CM as the AWJM specimens were less sensitive to temperature than the CM hole specimens because the increase in damage accumulation as a function of temperature was far more pronounced in the $\mathrm{CM}$ specimens. These results were in good agreement to that observed with the extensometer (cf. Figure 6-4 and Figure 6-5). However, regardless of the machining process used and for the same load, the damage rates calculated from the heat dissipation (Equation 47) are larger than those

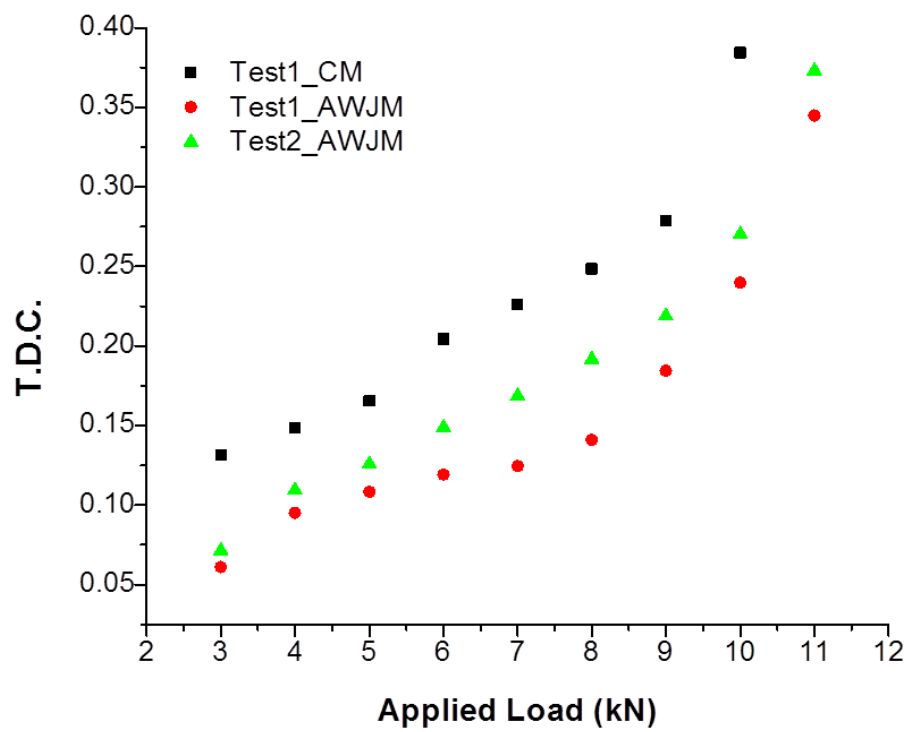

Figure 6-28 Comparison of the area of the portion having maximum temperature with the each loading stage from $3 \mathrm{kN}$ to $11 \mathrm{kN}$

calculated from the decrease of rigidity (Equation 1). These results are consistent with the findings of Toubal et al. [26] where a direct correlation was observed between the accumulated damage and the surface temperature. We can observe from Figure 6-28 that thermal threshold area was more for CM specimens than the AWJM specimens which was due to the surface topography created during machining processes. The fact was that the CM surface consists of 
more fiber pull-out and matrix damage areas which produced the highest temperature, and was more sensitive to fatigue crack initiation and propagation than striation marks created in the AWJM process as already explained in Section 6.2.1. So it means that the damage evaluation was the least in the case of AWJM specimens than the CM specimens as the number of cycles and load increased.

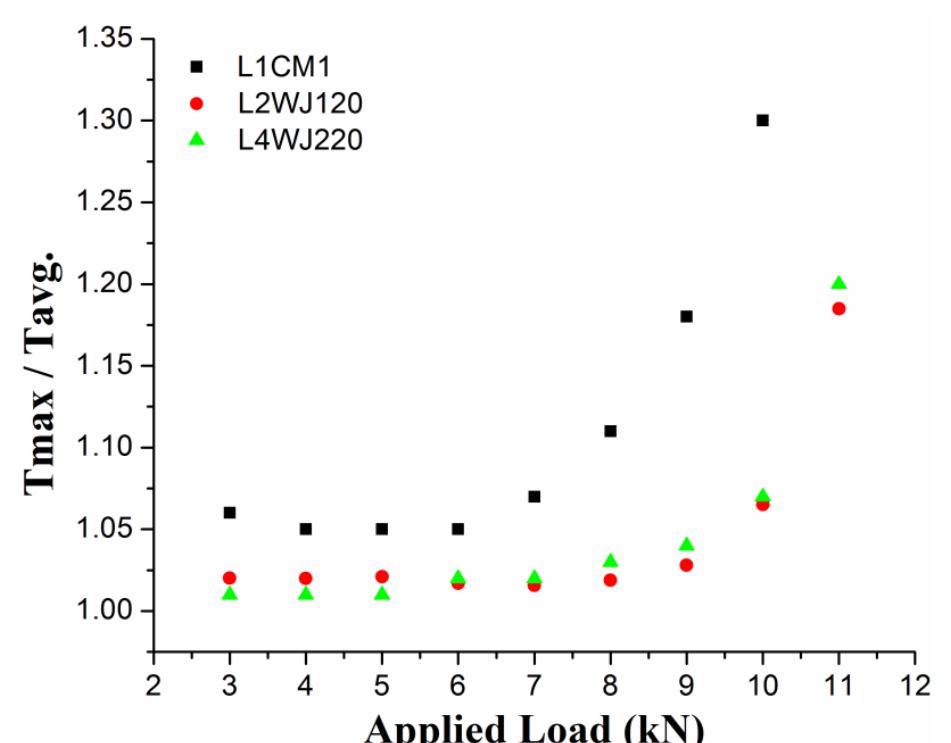

Figure 6-29 Comparison of the maximum and mean temperature against applied load for CM and AWJM specimens

The proposed TDC shown was very effective in predicting the damage accumulation by considering the thermal threshold in the selected zone as the increase in damage accumulation corresponded to the increase in temperature (Figure 6-21). The TDC can thus be used to predict damage accumulation under a given fatigue loading scenario and TDC further confirms the influence of the machining process on the mechanical behaviour of composite laminates.

\subsection{Surface Morphology of the Post-fatigued Angle ply Laminate}

\section{Specimen}

Microscopic observations were conducted after a destructive sectioning of a number of postfatigue tested specimens. Fractured specimens were cut accordingly to inspect the microstructure and the damage propagation in the machined surface. CF/Epoxy fractured specimens were examined using a scanning electron microscope (SEM) for the fractographic characterization of 
the machined surface after specimen failure during fatigue step loading. CM specimens loaded in axial tension failed in various modes as shown in Figure 6-30. All specimens failed at the gauge section at a small width as shown in Figure 6-31. Figure 6-30 illustrated the presence of the crack propagation phenomena due to the initial damages (fiber pull-out and matrix degradation) induced by the interaction between the machining tool and the carbon fibers as shown in the surface roughness and SEM observations of pre-fatigue specimens, shown in Figure 6-6 and Figure 6-7. These cracks were propagated due to the shear between the planes owing to the axial mechanical load in the composite with stacking sequence of $\left[ \pm 45^{\circ}\right]$. Figure 6-30 showed that the crack propagation resulted in the longitudinal and interface debonding.

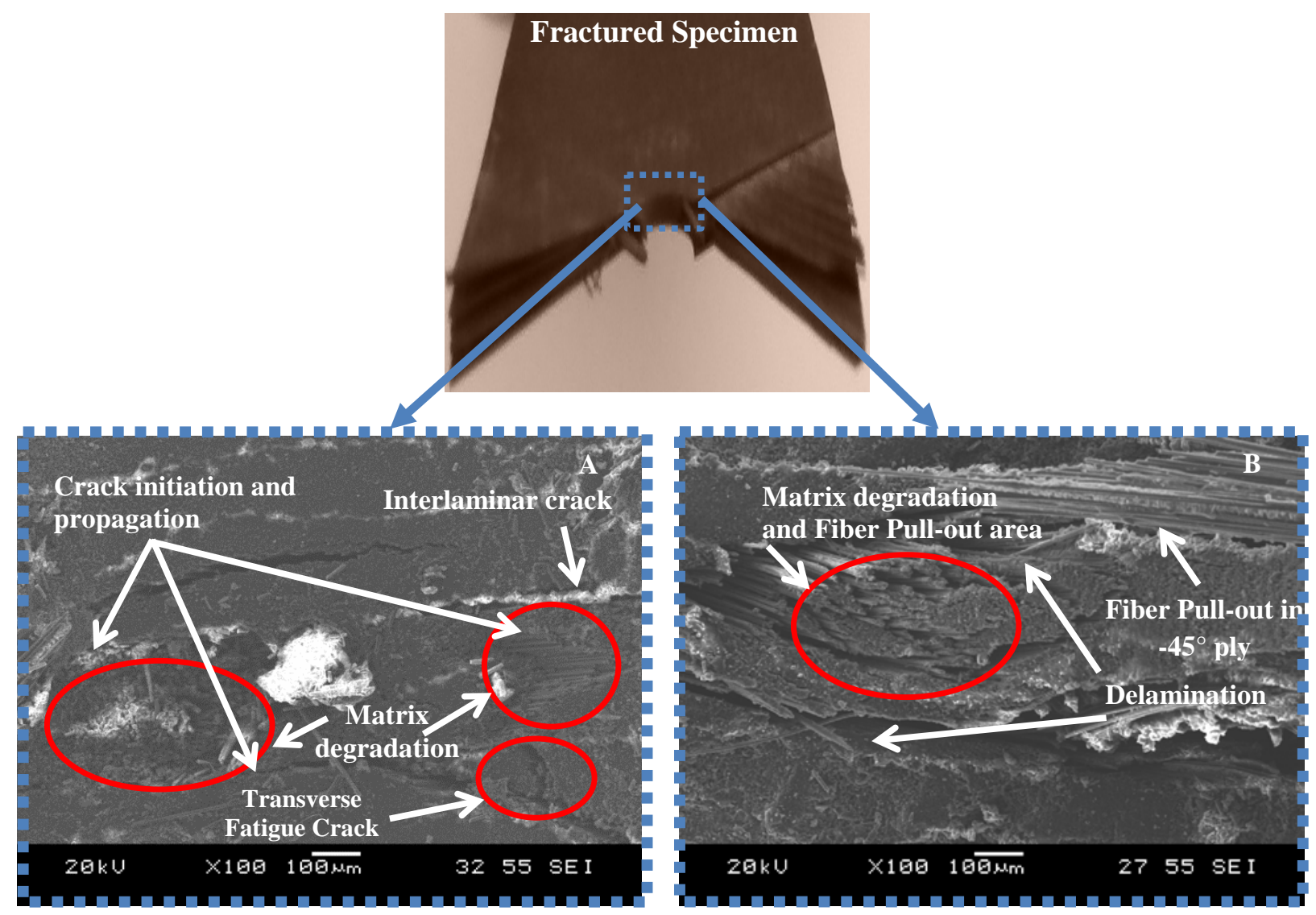

Figure 6-30 SEM micrograph showing a cross section of the circular hole of CF/Epoxy angle ply composite, circular hole obtained with the CM

The mechanically fatigued specimen also exhibited a transverse fracture (Figure 6-30a) in matrix degradation areas resulting from the drilling hole in the fiber's transverse direction. Figure 6-30b 
showed loose fibers and delamination in the plies from the fiber pull-out and matrix degradation locations as shown in the roughness images of the CM pre-fatigue specimen (Figure 6-6 and Figure 6-7).

These loose fibers subsequently reduced the strength of CM specimens. It is worth mentioning that the matrix degradation and fiber pull-out (induced by machining process) in CM specimens affected the fiber-matrix load transfer, and also affected interface bonding and loosened the pullout fiber consequently leading to significant material deterioration under tensile loading conditions which reduced the overall strength of the specimens. The different fracture modesgenerated under the fatigue cyclic load in the CM specimens led to reduction in various mechanical properties such as a decrease in the elastic modulus (Figure 6-1 and Figure 6-2), an increase in damage accumulation (Figure 6-4 and Figure 6-5), and a decrease in the endurance limit (cf. Figure 6-23). Hence, the major observable damages, fiber pull-out and matrix degradation, generated during the $\mathrm{CM}$ act as a stress concentrator for crack initiation and propagation at the surface of the machined hole and then gradually propagate width-wise toward the edge of the specimen, along the fiber direction i.e. $45^{\circ}$ (Figure 6-31), subsequently leading to the catastrophic failure of CM specimens and the final failure was oriented parallel to the fiber direction of the laminates (Figure 6-31), rather than across the width.

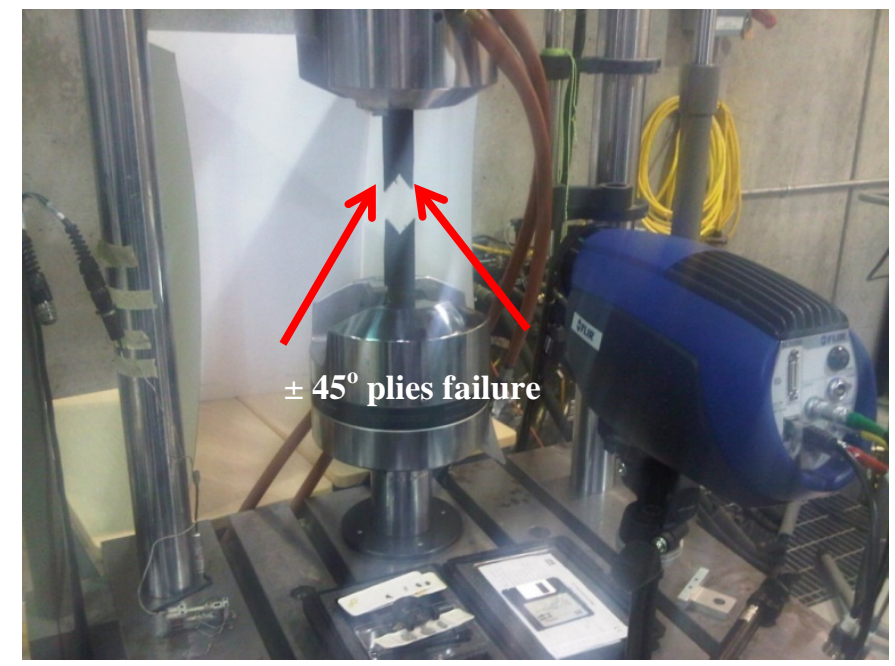

Figure 6-31 Fractured carbon epoxy composite laminate

\section{AWJM Specimen}


A post mortem surface observation of the AWJM specimens after fatigue loading was conducted using a scanning electron microscope. The SEM images presented in Figure 6-33 showed streak marks in the direction of the water-jet after fatigue loading. There was no visible fracture mode, crack or delamination observed in these images. However, internal component failure in composite specimens is normally initiated before any change in the macroscopic appearance is observed. The final failure of the AWJM specimen occurred according to three stages; in the first stage, there was friction between fibers/matrix and fibers/fibers.
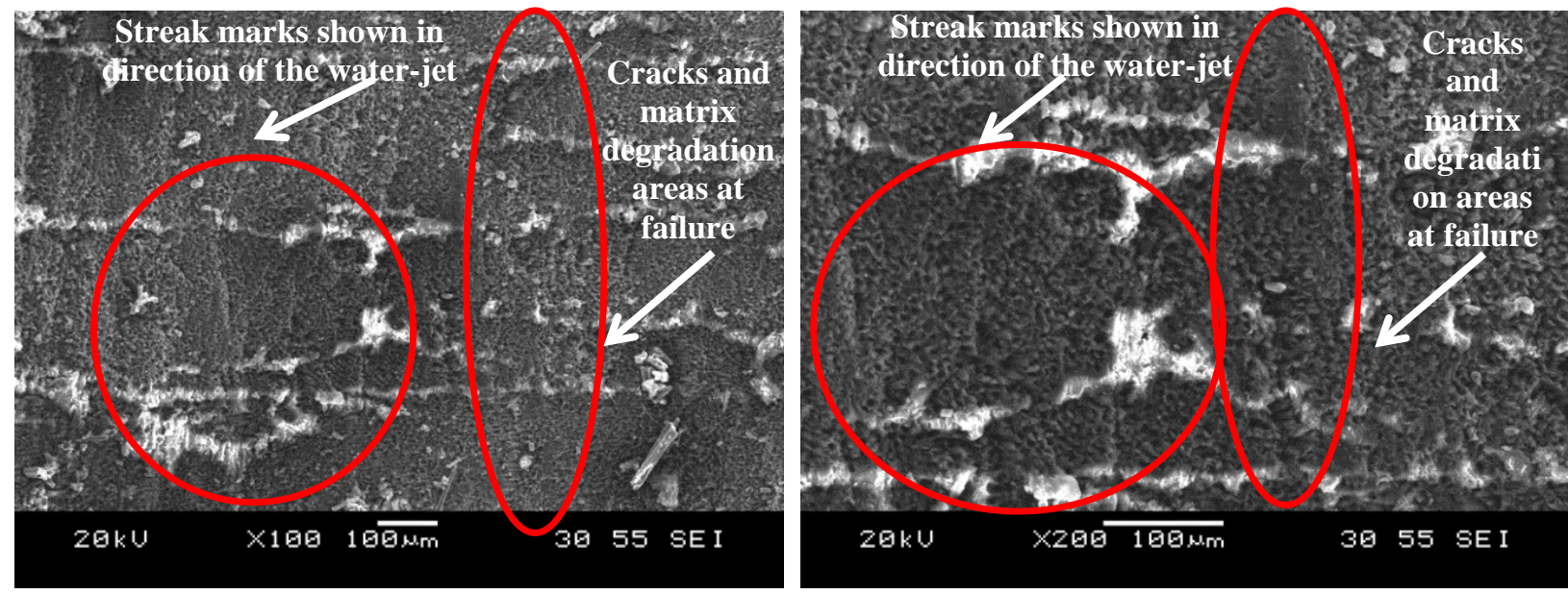

Figure 6-32 SEM micrograph showing cross section of circular hole of CF/Epoxy angle ply composite, circular hole obtained with AWJM

In the second stage, some microcracks developed in the matrix, resulting from the enhanced fiber/fiber and fiber/matrix friction. Microcracks propagated in the matrix and with the passage of time they reached the fiber/matrix interface. Due to the high strength of fiber, they change its direction and propagate along the fiber/matrix interface. With the increase in loading, the rate of the damage and the friction became more significant due to an increase in stress concentration at the fiber/materix interface. Finally, this stability was followed by an abrupt increase of the damage and a decrease in the stiffness of the AWJM specimens in stage three, which corresponded to the thermo-mechanical effects, plasticity and failure. This stage reached at $11 \mathrm{kN}$ load for AWJM when the angle ply specimen was tested at 5000 cycles. At failure, some cracks and matrix degradation areas were also observed on the machine surface (Figure 6-32). 


\subsection{Surface Morphology of the Post-fatigued Angle ply and Quasi Isotropic laminates}

Microscopic observations were conducted after a destructive sectioning of a number of postfatigue tested angle ply and quasi Isotropic laminates, tested at 10000 cycles to inspect their microstructure and damage propagation in the machined surface. The SEM images of the laminates having angle ply and quasi isotropic lay-ups, machined with the CM and AWJM processes were presented in Figure 6-33 and Figure 6-34 respectively. Specimens with different lay-ups and drilled with different machining processes experienced different damage mechanisms which can be related to the initial surface created after drilling processes.

Figure 6-33 ( $a$ and $b$ ) presented the failure mechanism of the angle ply laminate machined with the $\mathrm{CM}$ process and fatigue tested at 10000 cycles. Failure mainly occurred from transverse, interlaminar cracks and delamination. A transverse fatigue crack propagated from the matrix degradation area with the increased loading cycles which created stress concentration. Stress concentration from a weak matrix degradation area propagated to adjacent fibers. A further increased in the load resulted in a transverse fatigue crack in the CM angle ply laminate from the area generated during the machining process which resulted in the decrease of mechanical properties. Similarly, an interlaminar crack, generated from a pre-fatigue damage area, propagated with an increase in loading cycles and delaminated the interface between $-45^{\circ}$ and $+45^{\circ}$ ply which also contributed to the early failure of the CM specimens.

Figure 6-33 (c and d) presented the failure mechanism of quasi isotropic CM laminates; it showed failure mechanism almost similar to the CM angle ply laminate. Loose and broken $-45^{\circ}$ fibers can easily be seen (Figure 6-33c) and were generated from the degraded area initially produced during the machining process. This also resulted in the mid-plane delamination of the neighbouring ply $\left(0^{\circ}\right)$ with the increasing loading cycles. A similar phenomena caused the degradation of the mechanical properties of the $90^{\circ}$ ply and consequently the abrupt rupture of the specimen took place which at the time of failure also delaminated the $+45^{\circ}$ and $0^{\circ}$ plies as shown in Figure 6-33 (c and d).

AWJM specimens of both stacking sequences (Figure 6-34) showed streak marks and matrix degraded areas. Matrix degradation areas found on the machined surface resulted from the friction between fiber/fiber and fiber/matrix with the application of tensile cyclic loads. This friction, with the increasing applied load created stress concentration in the adjacent fibers and was mainly responsible for the failure of the AWJM specimens, as already discussed in detail in 

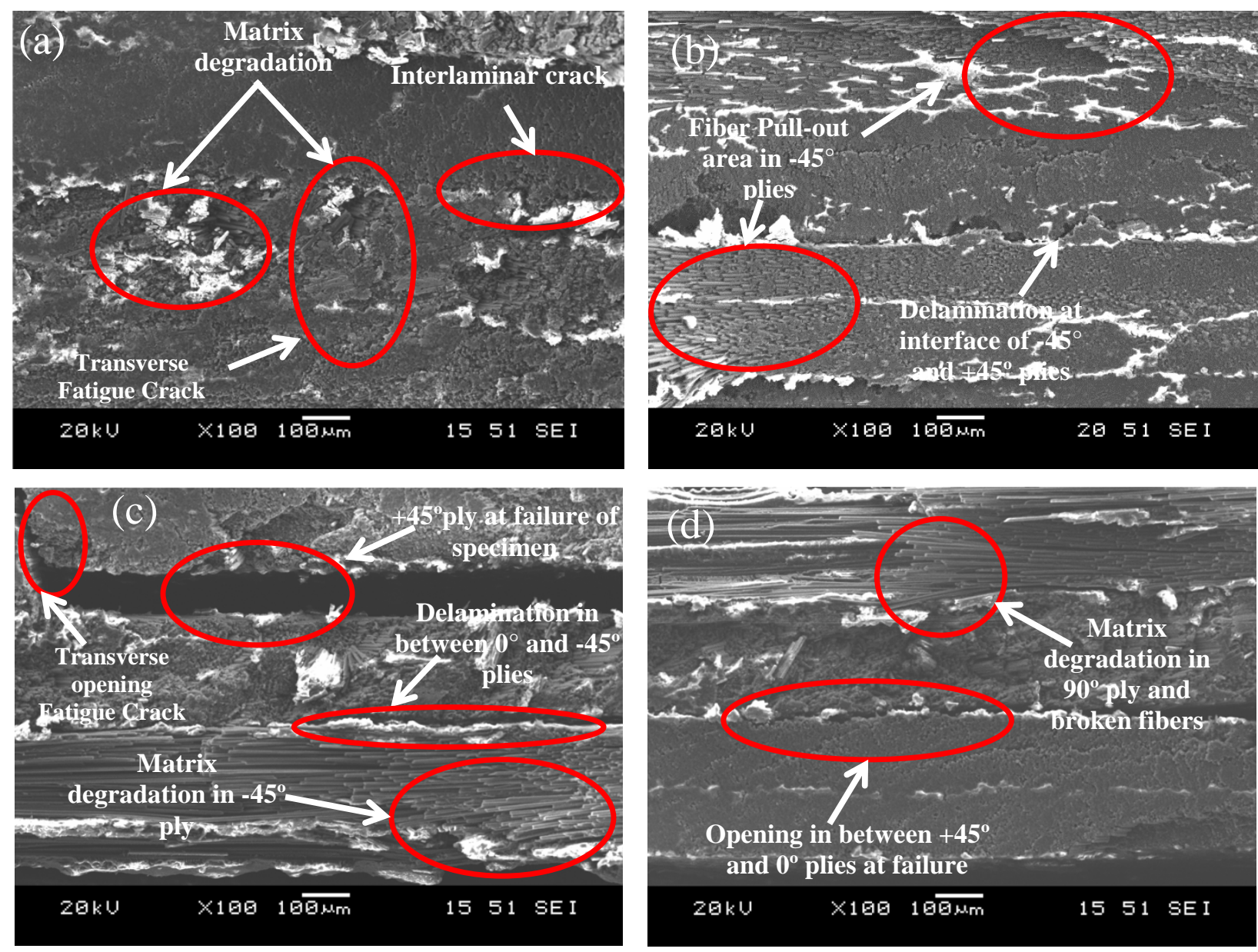

Figure 6-33 SEM micrograph showing the cross section of a circular hole of CF/Epoxy angle ply (Fig. $a$ and b) and quasi isotropic (Fig. $c$ and d) laminates, the circular hole was obtained with the $\mathrm{CM}$ process
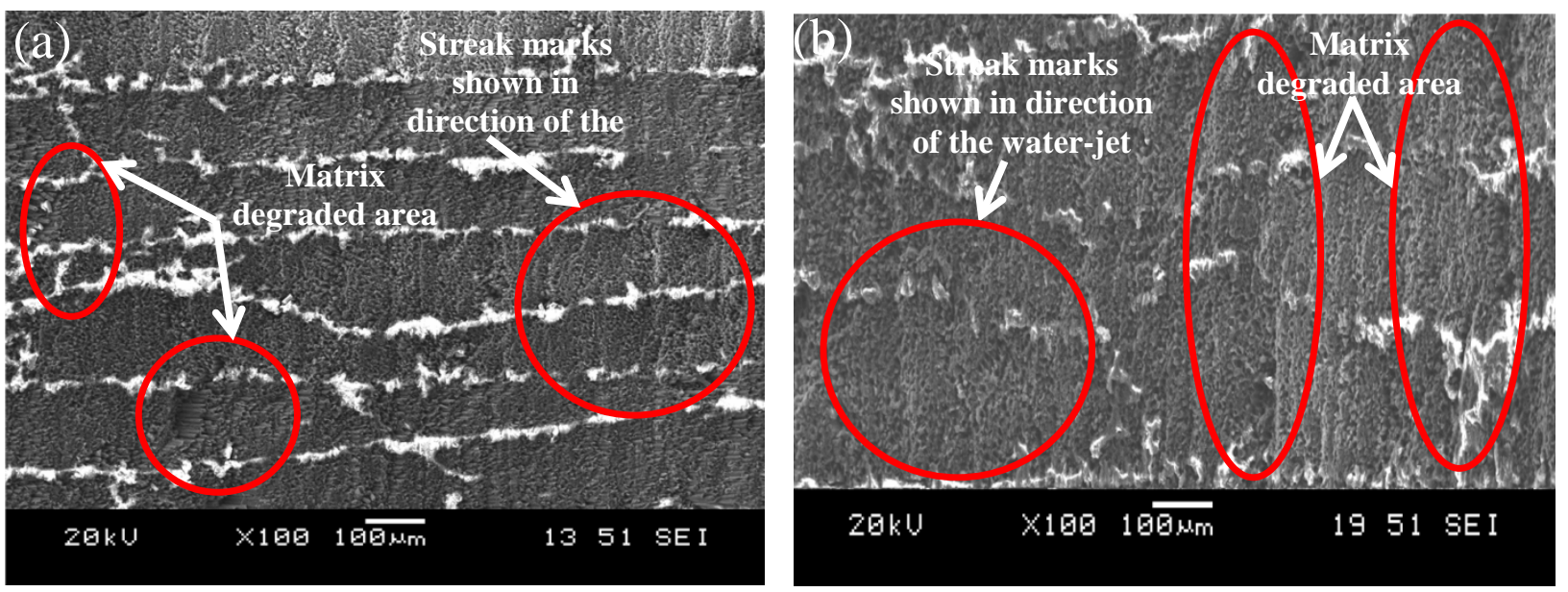

Figure 6-34 SEM micrograph showing cross section of circular hole of CF/Epoxy angle ply (Fig. a) and quasi isotropic (Fig. b) laminates, circular hole obtained with AWJM process 
Section 6.9. Furthermore, it was also noted that the matrix degraded area of the quasi isotropic laminate was more than the angle ply laminate, which was probably due to matrix dominated plies $\left(90^{\circ}\right)$.

\subsection{Summary}

$\mathrm{CF} /$ Epoxy angle ply and quasi isotropic specimens were investigated for surface damage at the wall of the machined hole after drilling with two machining processes, and were subsequently put to fatigue tests at 5000 and 10000 cycles to evaluate the influence of hole surface defects on the mechanical performance of CFRP specimens.

Based on the experimental analysis, the following were observed:

- Both specimens have shown similar damage for loads less than $46 \%$ of UTS. For loads above this value, the damage was more significant for the specimens drilled using conventional machining. The damage accumulation in the case of conventional drilled specimens was higher than the one with AWJM specimens. This difference can be related to the initial surface integrity after machining induced by the difference in the mechanism of material removal between the two processes used. This is supported by the surface topography (roughness) tests as shown in Figure 6-6 which showed that surface texture plays an important role in the damage accumulation of the material as a surface produced by the CM process has a poorer damage behaviour than AWJM machined surface. It was observed that, when the conventional machining process was used, the maximum damage due to fiber pull out was observed when plies were oriented at $-45^{\circ}$ compared to the direction of the cutting speed. However, the minimum damage was located in the zone where the fibers form an angle of $+45^{\circ}$ compared to the direction of the cutting speed. The angle between the fiber orientations and the cutting speed direction influenced the mechanism of material removal and the mode of the rupture. So it was concluded that the damage was dependent upon the machining process used, which was also confirmed from the analysis of the SEM images of the post-fatigue fractured specimens. These specimens confirmed that the fiber pull-out and matrix degradation areas act as a stress concentrator for crack initiation and propagation at the machined surface of the hole in CM specimens. Furthermore, damage accumulation in CM specimens, in the case of angle ply was more than the quasi isotropic laminates because the quasi isotropic laminates consist of fiber 
dominated plies. The effect of the test parameters were also studied and were correspondingly compared and found that increasing tests parameters decreases the fatigue life for all of the tested specimens.

- Surface roughness images showed that the machined hole surface of CM laminates showed visibly distinct regions of fiber pull-out and epoxy matrix degradation, while those of AWJM laminates showed striation marks in the direction of water-jet and craters defects due to the impact of the abrasives on the fibers. No regions of fiber pull-out or thermal matrix damage was evident on the AWJM hole surface. It means that the fiber pull-out and matrix degradation were responsible for greater values of stress concentration sites in CM specimens compared to the striation marks created in AWJM specimens. Surface roughness parameters $(R a$ and $R v)$ indicated that AWJM and CM holes have the same surface roughness values, although the type and the form of the damage generated by each machining process was completely different. Hence the roughness criteria generally used to quantify the quality of machined surfaces are not representative of the mechanical performances of the composite material. In addition, they cannot be representative of the subsurface damage. One can conclude that unlike metallic materials, the criterion used for quantifying the quality of machining based on the average roughness $(R a)$ is not suitable for composite laminates.

- It was concluded that the maximum temperature profiles in the area surrounding the hole (i.e., studied area) follow the same evolution as the damage profiles for both machining processes. However, the presence of defects induced by the cutting tool in conventional machining (pull-out of fibers and degradation of the resin) provoke more heat dissipation than ridges and crater defects observed on the wall of the holes machined by the abrasive water-jet machining process. Infrared thermographic (IR) images showed less heat dissipation in $[90 /+45 / 0 /-45]_{S}$ than $\left[ \pm 45^{\circ}\right]_{2 S}$ laminates, however when comparing the machining process, AWJM dissipated less heat than CM specimens for all tested laminates. Quasi isotropic laminates failed in a more brittle manner and showed a linear mechanical response during the fatigue life than angle ply laminates therefore it dissipated less heat than angle ply laminates. These results corresponded well to the damage accumulation in these laminates. 
- IR thermography can be effectively used for accessing the effect of different machining processes on the mechanical behaviour of CFRP during fatigue testing. IR thermography can be effeciently used for in-service monitoring of the crack initiation and propagation. Moreover, heat dissipation during fatigue loading can be used as a damage criterion for the fast monitoring of in-service crack initiation and propagation. We can conclude that heat dissipation can be presented as a superb technique to ascertain mechanical behaviour of the CF/Epoxy composite laminates.

- For all specimens, the change in temperature vs stress graphs showed a sudden change in the slope of the temperature curve when the endurance limit was attained and after that point the temperature increased rapidly because of the propagation of the microcracks and increased damage accumulation. However, below the endurance limit small temperature changes were observed which was due to the thermoelastic effect. CM specimens underestimated the endurance limit by 10-15\% when compared to the AWJM specimens that have no fiber pull-out and matrix degradation areas. This showed that endurance limit is strongly influenced by the surface texture created after machining.

- A thermographic damage criterion (TDC) has been defined to describe the two types of machining processes which quantitatively described the damage accumulation, correlating it with the thermal threshold area. By analyzing the heat dissipation during the application of cyclic loading, it was possible to evaluate the damage accumulation. The damage obtained from the TDC was larger than those calculated from the decrease of rigidity. The proposed thermographic damage criterion was very effective in predicting the damage evolution by considering the thermal threshold. This criterion showed that the increase in the thermal threshold area was responsible for the earlier failure of the CM specimen. It was observed that fatigue failure shown by an increase in damage accumulation and an increase in the thermal threshold area resulted in deterioration in the mechanical properties of CM specimens, which were strongly dependent on the surface texture. The surface texture (fiber pull-out and matrix degradation area) was responsible for the initiation of stress concentration sites, increasing the thermal threshold area and decreasing the mechanical properties of the CM specimens while AWJM specimens generated less temperature and damage. Thus the degree of fatigue life improvement by AWJM specimens is found to be dependent on the circular hole surface created during 
the machining process. This study showed that the fatigue improvement can be achieved by selection of appropriate machining processes. Besides damage accumulation, heat dissipation and the endurance limit analysis, the TDC further confirmed the difference between AWJM and CM specimens.

Finally, it can be concluded that the use of abrasive water-jet machining process will result in a more efficient utilization of composite laminates manufactured from different lay-ups for use in aerospace industries. All observations of this study indicated that the choice of the process of machining has an important role on the functionality of the structural parts. 


\section{CHAPTER 7. FATIGUE TESTING OF CFRP ASSEMBLY}

In this chapter, first the damage assessment of bolted joint composite assembly is presented. Additionally, heat dissipation from assembly is assessed using infrared thermography. In the next section, the high cyclic fatigue strength is presented to find the fatigue life followed by the analysis of assembly fractured specimens to identify the failure mechanism of the conventional and abrasive water-jet machined specimens. At the end of the chapter, a brief summary of this chapter is presented.

\subsection{Fatigue damage assessment}

Damage accumulation during the cyclic loading was calculated from the data obtained from fatigue experiments on assembly specimens using Equation 1. The influence of machining processes on the damage accumulation for assembly specimens of stacking sequence [90/+45/0/$45]_{S}$ and $\left[ \pm 45^{\circ}\right]_{2 S}$, in each step load during fatigue testing is shown in Figure 7-1 and Figure 7-2 (Appendix A- 10), respectively.

For quasi isotropic assembly specimens, the cumulative damage, for a given value of the applied load inferior or equal to $40 \%$ of UTS, was uniform and increased gradually for all specimens tested and for any process of machining used. For exemple, when the applied load was equal to $30 \%$ of the UTS, the accumulated damage of the CM specimens and for the AWJM specimens was identical and estimated around 20\% (cf. Figure 7-1). With the increase of the applied load, it was observed that the accumulated damage was strongly affected by the machining process. It was noticed that, with the increase of the applied load from $40 \%$ to $65 \%$ of UTS the accumulted damage increased rapidly for all specimens tested and for any process of machining used. However, the accumulated damage monitored on specimens machined by the $\mathrm{CM}$ process reached up to $58 \%$ compared to only $38 \%$ damage for the assembly made of the specimens machined with the AWJ process. Finally, after a loading range of between $65 \%$ and $75 \%$ of UTS, a sudden augmentation of the accumulated damage till the failure of the assembly made of the CM process was observed. Nevertheless, when the AWJM specimens were considered, the damage increased till $50 \%$ when the applied load was equal to $75 \%$ of UTS and with the increasing of the loading till $80 \%$ of UTS the accumulated damage reached the maximum (80\%) 
at the 10000 cycles and when the applied load was equal to $85 \%$ of UTS, a sudden failure was observed at 2000 cycles.
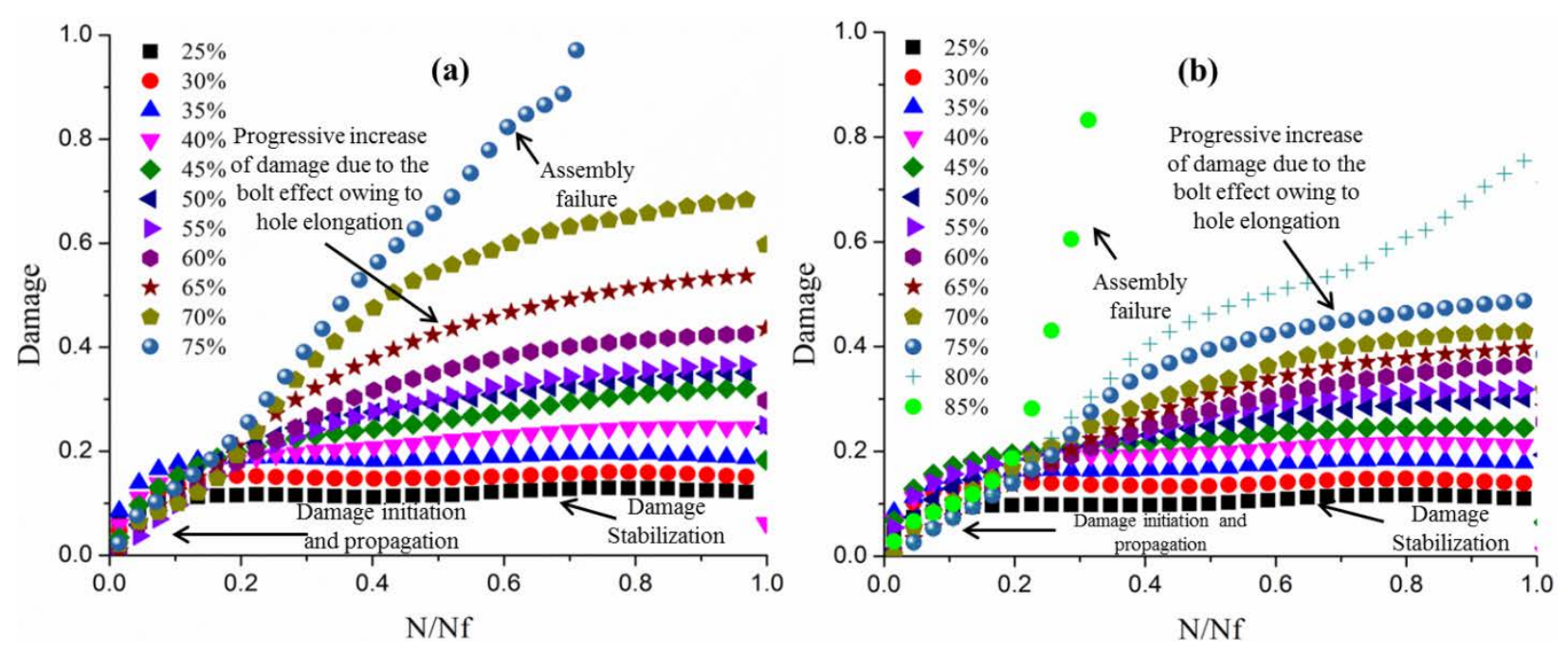

Figure 7-1. The evolution of the damage for various level of loading for CF/Epoxy quasi isotropic assembly specimens. Specimens machined with (a): the CM process and (b): the AWJM process. Condition of the tests: frequency of $10 \mathrm{~Hz}$ and number of the cycles for each level of loading is 10000

In fact from Figure 7-1 and Figure 7-2, it is clear that the damage increased in two stages when the load was inferior or equal to $60 \%$ and $70 \%$ of UTS for the $\left[ \pm 45^{\circ}\right]_{2 S}$ and quasi isotropic assembly specimens respectively, when the AWJM process was considered. In the first stage, a sudden increase in damage was observed while in the second stage, a stabilization in damage was noticed till the end of 10000 cycles in each step loading. Damage in the first stage can be associated to the matrix cracking due to the effect of the fastener and the second stage can be explained by the saturation in the damage. However, for the CM process, the two stages were observed when the load was inferior or equal to $55 \%$ and $60 \%$ of UTS for the $\left[ \pm 45^{\circ}\right]_{2 S}$ and quasi isotropic assembly specimens. In addition, with CM specimens, the first stage of damage was also combined with the crack propagation from the locations of the initial damages induced by the machining process, which is evident from the higher damages in each load stage in CM than AWJM specimens, as discussed above.

In the case of assembly specimens when the stacking sequence of $\left[ \pm 45^{\circ}\right]_{2 S}$ was considered, the behaviour of damage accumulation was uniform till 55\% and $60 \%$ load of UTS, when the CM (Figure 7-2a) and AWJM (Figure 7-2b) processes were used, respectively. The assembly 
specimen with stacking sequence of $\left[ \pm 45^{\circ}\right]_{2 S}$ showed that, from the first loading (25\% of UTS) a higher damage accumulation was recorded when specimens were machined with the CM process compared to the damage accumulation of the specimens machined with AWJM process. This gap was estimated around $10 \%$ for the CM and AWJM specimens. The higher damage at the first
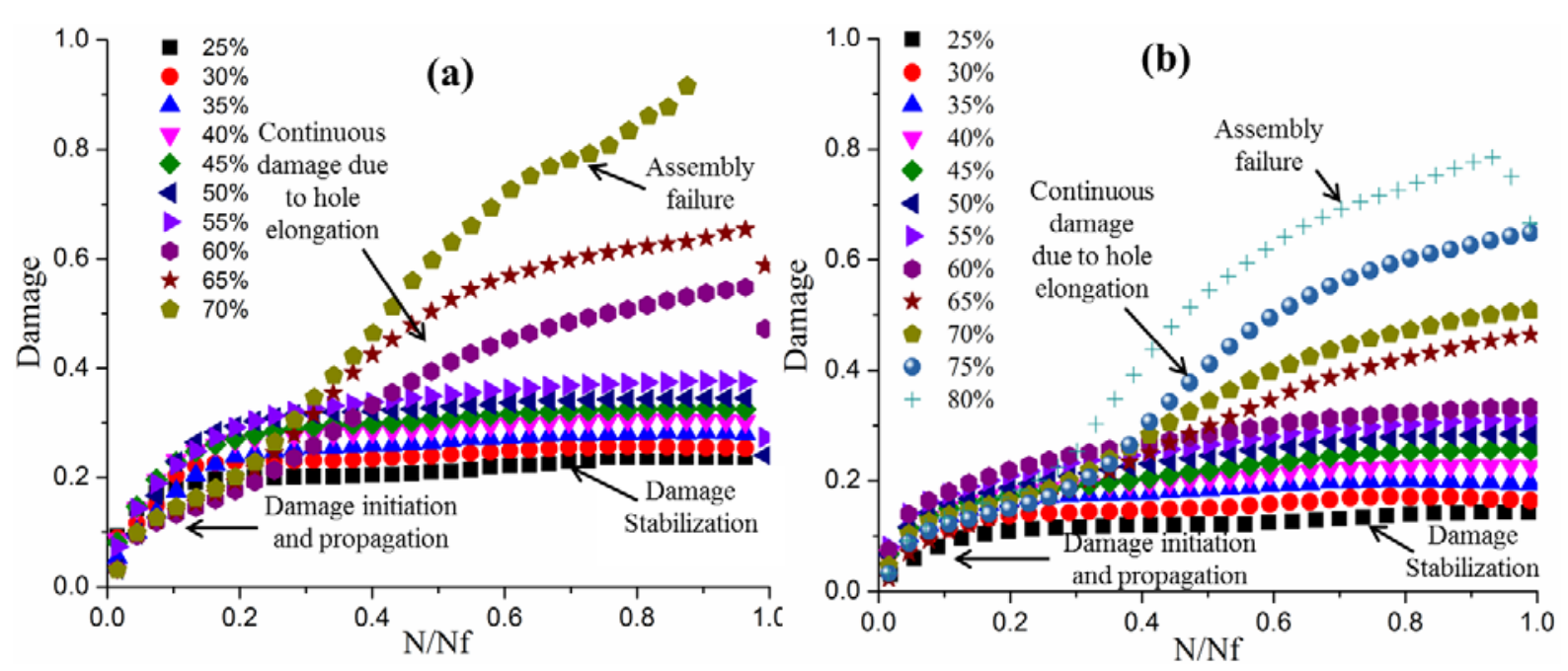

Figure 7-2 The evolution of the damage for various level of loading for CF/Epoxy angle ply assembly specimens. Specimens machined with (a): the CM process and (b): the AWJM process. Condition of the tests: frequency of $10 \mathrm{~Hz}$ and number of the cycles for each level of loading is 10000

loading in the CM specimen was due to two reasons. Firstly, due to the initial damages induced by the machining process, secondly, the contact of bolt-hole surface generated frictional forces which resulted in the cracking of the matrix. After the first loading, damage increased at a constant rate in the CM specimen until loading of 55\% of UTS $(3.95 \mathrm{kN})$. In this case, the accumulated damage on assembly made of the CM process was around $40 \%$. However, in the specimen drilled with the AWJM process, the accumulated damage was 31\% at the same loading. It is important to notice that the damage rapidly increased to $57 \%$ in the CM assembly at $60 \%$ load of UTS $(4.31 \mathrm{kN})$ and it failed at $70 \%$ load of UTS $(5.03 \mathrm{kN})$ after less than 8500 cycles. However at $60 \%$ load of UTS and at same number of cycles, the AWJM specimen continued to withstand cyclic stresses and the damage was less than $30 \%$ and a difference of around $60 \%$ in damage was noted when compared to CM assembly at same load and number of cycles. However, when the AWJM assembly lasted all 10000 cycles without failure, the accumulated damage increased moderately to $34 \%$, but after that, damage increased rapidly to 
$65 \%$ at $75 \%$ load of UTS and it further increased continuously till failure at $80 \%$ load of UTS $(5.74 \mathrm{kN})$. In addition, it has been noticed that the rate of damage increases in the damage stabilization stage is comparatively less than the specimens with a quasi isotropic stacking sequence.

Damage behaviour discussed above showed that the fatigue failure of the assembly specimens occurred at a lower load level than single component during fatigue test as was observed in Section 6.2. However, AWJM specimens sustained a higher load before failure than conventional machined assembly specimens of both stacking sequecnces.

To better understand that why the process of machining has an important influence on the accumulated damage under fatigue loading, the machined surface of the wall of the holes have been investigated by performing surface roughness tests of the pre-fatigue specimens with the help of a NANOVEA 400 series surface profilometer. The surface roughness $(R a)$ was measured according to ISO 4287/1 standard using NANOVEA 3D software. Surface roughness images obtained from a NANOVEA 400 series profilometer were shown in Figure 7-3 and Figure 7-4 for CM and AWJM specimens having a stacking sequence of $[90 /+45 / 0 /-45]_{S}$ and $\left[ \pm 45^{\circ}\right]_{2 \mathrm{~S}}$ respectively. Surface roughness $(R a)$ values represented in Table 7-1 showed almost the same roughness values for all specimens of both stacking sequences. However, during the fatigue tests, the tolerance of damage of these assembly specimens was completely different. For the CM specimens and for both stacking sequence, fiber pull-out and matrix degradation areas were observed with the crack density of 8.77 and 5.39\% (Table 7-2). However, AWJM assembly specimens showed streak marks in the direction of the displacement of the water jet having a crack density of 1.68 and $0.62 \%$ for a stacking sequence of $\left[ \pm 45^{\circ}\right]_{2 S}$ and $[90 /+45 / 0 /-45]_{S}$, respectively. Hence, the higher damage accumulation in all of the CM assembly specimens was characterized by a higher crack density and fiber pull-out while streak marks and less crack density in AWJM assembly specimens produces less damage accumulation. Surface roughness images were also confirmed by scanning electron microscopy images shown in Figure 7-3 (a1 \& b1). The position of the damage (fiber pull-out and matrix degradation) induced by the CM process was strongly influenced by the stacking sequence of the considered specimens. However, when the AWJ machining was used, the form of the generated damage was not impacted by the stacking sequence of the tested specimens. This can be explained by the fact that, when the AWJM process was used, the mechanism of the material removal was the erosion combined with 
the abrasion. However, with the CM process, the mechanism of the material removal is more complex and depends on the relative angle between the cutting speed and the fiber orientation of the ply.

From topographies of the different machined surfaces presented in Figure 7-3 and Figure 7-4 it is clear that the field of contact pressure which was developed between the wall of the hole and the bolt was strongly influenced by the microscopic state of the machined surface. Therefore, the local stress (Bolt/Wall of the hole) was strongly superior to the applied macro stress. Hence with the increase in the loading steps (applied macro stress), the damage was continuously increased
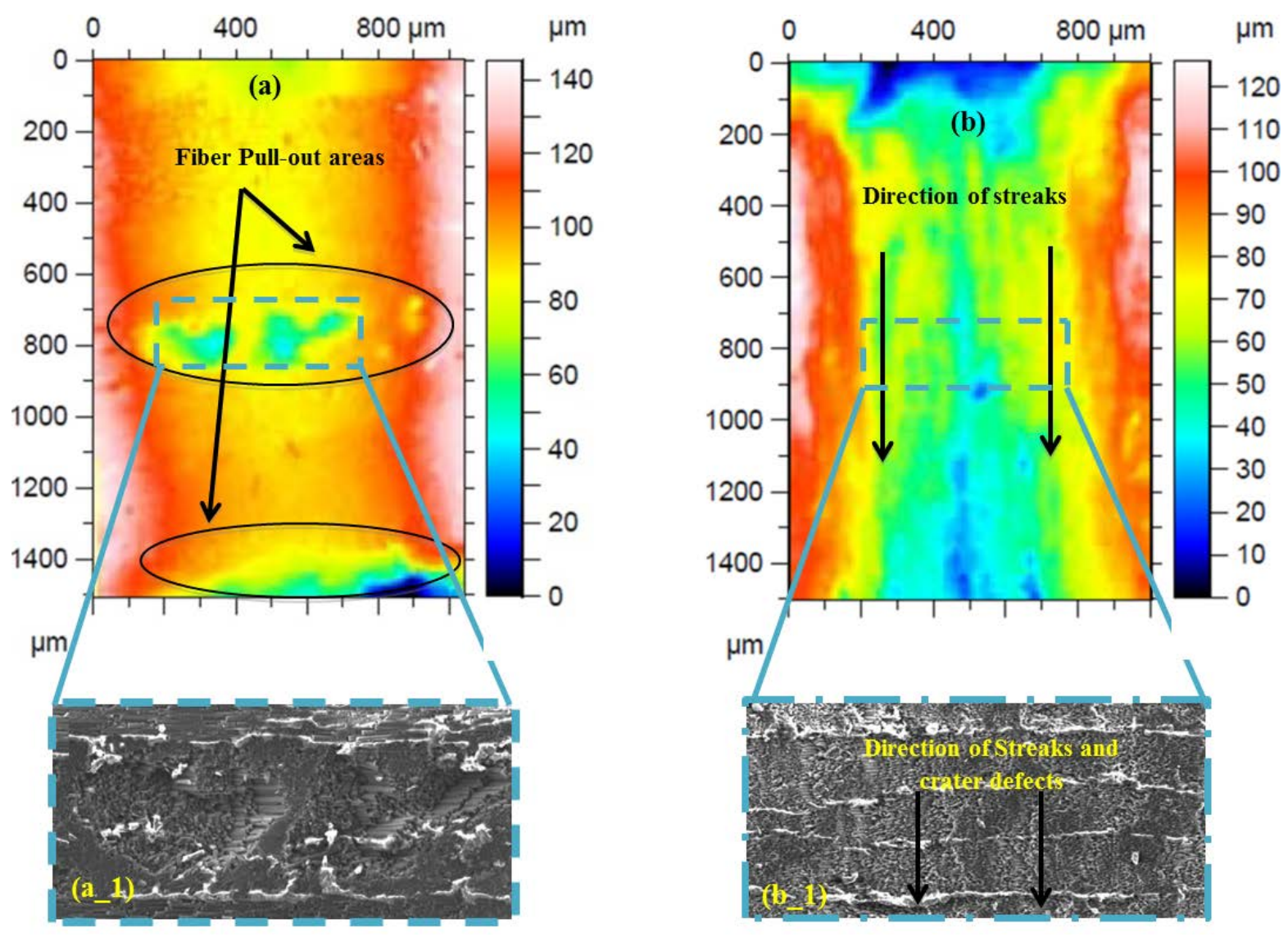

Figure 7-3 Surface Roughness images showing cross section of circular hole of pre-fatigue Carbon/Epoxy quasi isotropic laminate, circular hole obtained with (a) CM and (b) AWJM processes, while Figure (a_1) and (b_1) shows SEM images of the selected locations.

throughout 10000 cycles in each loading step, which favours the hole elongation in all assembly specimens as shown step by step in the images of Figure 7-5 and Figure 7-6 obtained from an infrared thermographic camera. Further, the elongation of the hole in assembly specimens 
occurred due to the bolt tilting which created more clearance between the hole and bolt surface. During the last stages of the step loading, the bolt bended with the surface of the hole due to the clearance created in previous step loading. This created contact pressure between the bolt and hole surface, which increased the strain rate and was responsible for the damage created around hole. Consequently, the rate of damage accumulation increased progressively in the last step loading stages when compared to the sudden increase and then stabilization of the damage accumulation in the initial step loading stages, as reflected by the slopes of the curves represented in Figure 7-1 and Figure 7-2.

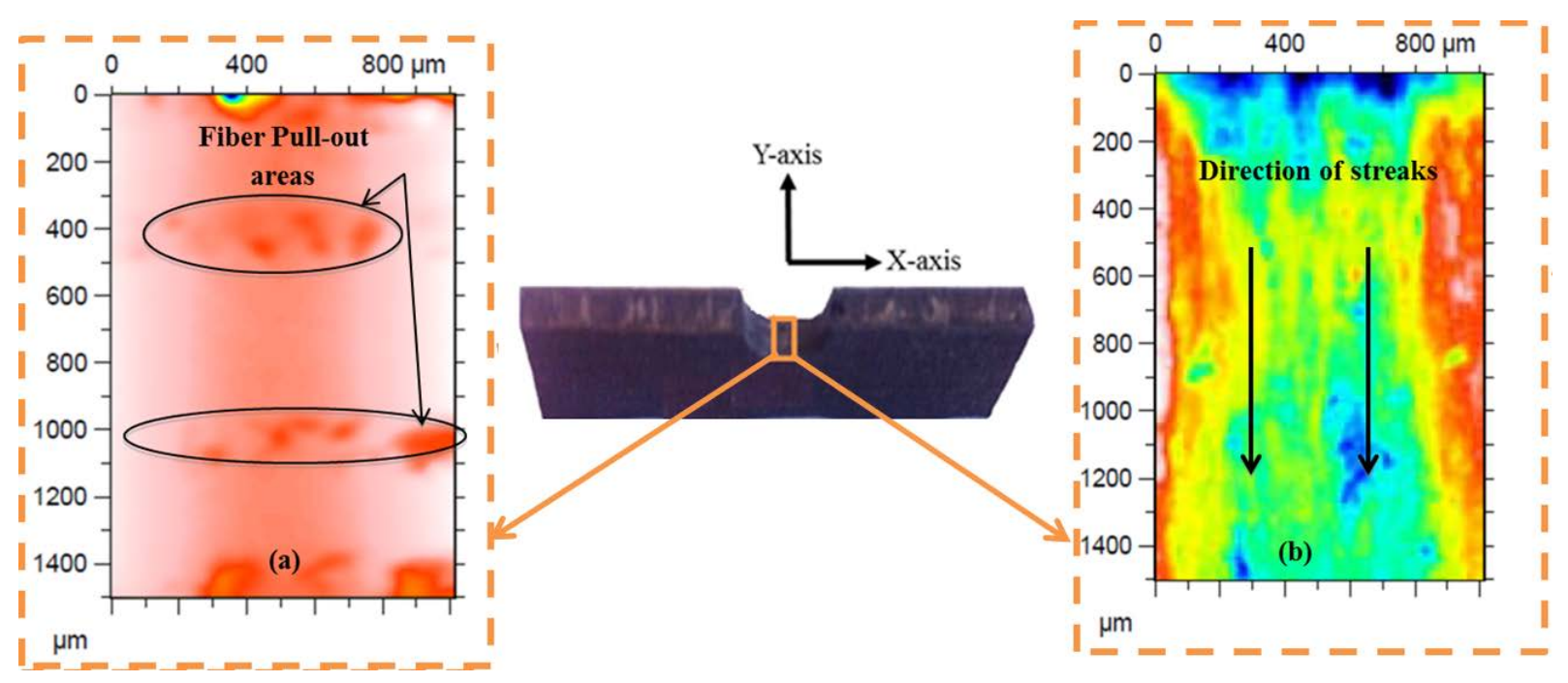

Figure 7-4 Surface Roughness images showing angle ply of circular hole of pre-fatigue Carbon/Epoxy angle ply lay-up laminate, circular hole obtained with (a) CM (b) AWJM processes

It is important to mention that, although the surface roughness " $R a$ " obtained from the two machining processes were almost similar, however the fatigue behaviour of these specimens was entirely different. The roughness criteria $(R a)$ currently using in industry in order to characterize the quality of the composite machined surface (drilled or milling) is not credible. These results are in good agreement with those presented in Section 6.2.1 [111] and from literature by [112], which were concerned with the drilling and trimming of the specimens obtained with different process of the machining and were subjected to the fatigue loading. In addition, in the work of Minnetyan et al. [7], authors have used single bolted 48 plies graphite/epoxy specimen with a stacking sequence of $[0 / \pm 45 / 90]_{6 S}$ and holes were drilled with a conventional tool. It was found 
that the damage commenced in $90^{\circ}$ plies by matrix cracking, adjacent to the bolt. The rate of damage decreased in the damage stabilization stage and then a sharp decrease in stiffness was found when through-the-thickness local fracture takes place. Similar results about the initial damage progression were observed in the composite laminates investigated in present research.

Table 7-1 Surface roughness of pre-fatigue CF/Epoxy assembly specimens drilled with CM and AWJM processes.

\begin{tabular}{lcccc}
\hline & \multicolumn{3}{c}{ Surface roughness $(\mathbf{R a}) \boldsymbol{\mu m}$} \\
\cline { 2 - 5 } \multicolumn{1}{c}{ Description } & $\begin{array}{c}\text { Stacking sequence of angle ply } \\
\text { lay-up laminate }\end{array}$ & $\begin{array}{c}\text { Stacking sequence of quasi } \\
\text { isotropic laminate }\end{array}$ \\
\cline { 2 - 5 } & $\begin{array}{c}\text { Surface roughness } \\
(\mathrm{Ra}) \mu \mathrm{m}\end{array}$ & $\begin{array}{c}\text { Surface } \\
\text { roughness } \\
(\mathrm{Sa}) \mu \mathrm{m}\end{array}$ & $\begin{array}{c}\text { Surface } \\
\text { roughness } \\
(\mathrm{Ra}) \mu \mathrm{m}\end{array}$ & $\begin{array}{c}\text { Surface } \\
\text { roughness }(\mathrm{Sa})\end{array}$ \\
\hline $\begin{array}{l}\text { Conventional machined } \\
\text { specimens }\end{array}$ & $4.06 \pm 0.34$ & 12.03 & $4.54 \pm 0.51$ & 14.60 \\
$\begin{array}{l}\text { Abrasive water-jet } \\
\text { machined specimens }\end{array}$ & $3.59 \pm 0.26$ & 14.20 & $4.16 \pm 0.17$ & 14.60 \\
\hline
\end{tabular}

Table 7-2 Crack density (\% area of crack) of CF/Epoxy pre-fatigue specimens drilled with CM and AWJM processes. Crack density was calculated using image analysis software.

\begin{tabular}{lcc}
\hline \multicolumn{1}{c}{ Description } & \multicolumn{2}{c}{ Crack density (\% area of crack) } \\
\cline { 2 - 3 } & $\begin{array}{c}\text { Stacking sequence of } \\
\text { angle ply lay-up laminate }\end{array}$ & $\begin{array}{c}\text { Stacking sequence } \\
\text { of quasi isotropic } \\
\text { laminate }\end{array}$ \\
\hline Conventional Machined specimens & $8.77 \pm 2.14$ & $5.39 \pm 1.71$ \\
Abrasive water-jet machined specimens & $1.68 \pm 1.96$ & $0.62 \pm 0.48$ \\
\hline
\end{tabular}

However, a progressive increase in damage was observed before the final failure owing to the elongation of the hole, as explained earlier. In another study, Starikov et al. [92] showed that when a high load was applied on the bolted joint specimen, various severe damages were produced around the bolt holes. Quick changes in damage development were observed between 
bolt and the adjacent hole surface. The significant effect of friction and hole wear on the fatigue behaviour of the bolted joint specimen was found. In the present work, in addition to the bolthole friction effect, it was also shown that the damage produced around the hole was also affected by the initial surface integrity induced by the difference in the mechanism of material removal, as illustrated in Figure 7-3 and Figure 7-4. Saunders et al. [102] found that erosion process was affected somehow by the stacking sequence of the laminates while in the current study, different machining processes were considered and it was observed that the conventional machining process is strongly influenced by the stacking sequence of the tested assembly specimens.

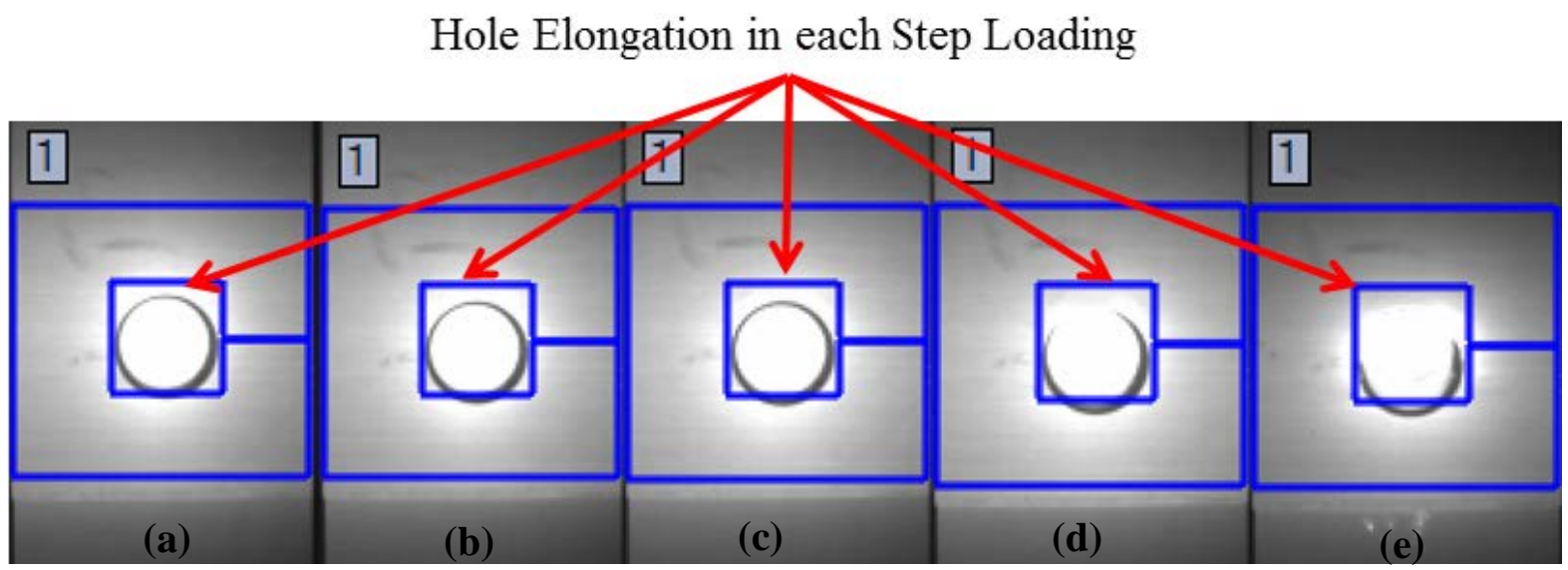

Figure 7-5 Hole elongation during fatigue testing of AWJM angle ply lay-up assembly specimen for loads of (a) $65 \%$ (b) $65 \%$ (c) $70 \%$ (d) $75 \%$ (e) $80 \%$ of UTS.

Hole Elongation in each Step Loading

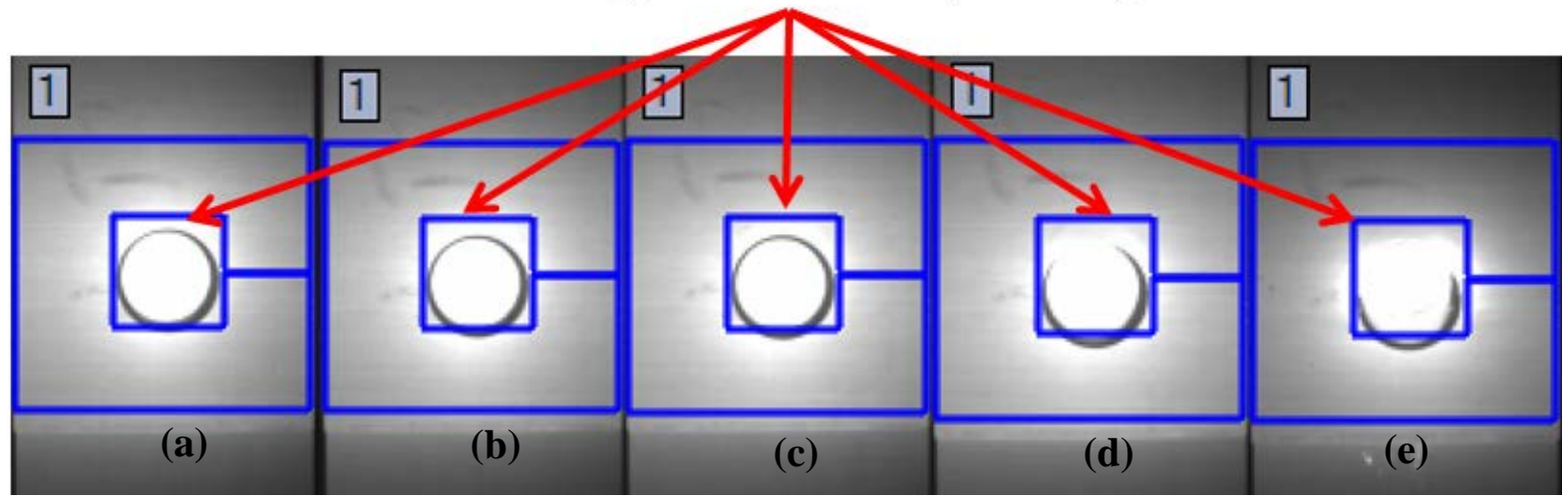

Figure 7-6 Hole elongation during fatigue testing of AWJM quasi isotropic assembly specimen for loads of (a) $65 \%$ (b) $70 \%$ (c) $75 \%$ (d) $80 \%$ (e) $85 \%$ of UTS. 


\subsection{Damage assessment using infrared thermography}

An infrared thermographic camera was used to obtain heat dissipation by continuously recording the surface temperature for each step loading stage during the fatigue test. Figure 7-7 to Figure 710, shows the temperature profile of joint-bolted composite laminates with a stacking sequence of $\left[ \pm 45^{\circ}\right]_{2 S}$ and $[90 /+45 / 0 /-45]_{S}$ and for the both processes of machining used (CM and AWJM). These images were obtained for 25, 45 and $65 \%$ load of UTS and the final image shows the temperature profile just before failure (i.e. 70 and $80 \%$ of UTS for CM and AWJM assembly). Figure 7-7 and Figure 7-8 shows that initially at 25\% load of UTS, temperature was almost constant $\left(26^{\circ} \mathrm{C}\right)$ for AWJM and CM angle ply assembly specimens. With the increasing of load to $45 \%$ of UTS, temperature increased slowly $\left(38^{\circ} \mathrm{C}\right)$ in AWJM specimens than $\mathrm{CM}$ specimens $\left(43^{\circ} \mathrm{C}\right)$. Specimens machined with AWJM process reached to $61^{\circ} \mathrm{C}$ at $65 \%$ load of UTS. Conversely, specimens machined with the $\mathrm{CM}$ process underwent a rapid increase of temperature with the increase of the load and reached $75^{\circ} \mathrm{C}$ at $65 \%$ load of UTS and the temperature further increased to $110^{\circ} \mathrm{C}$ at $75 \%$ load of UTS when $\mathrm{CM}$ specimen failed. However, the specimen machined with AWJM failed at 85\% load of UTS and the recorded temperature was $88^{\circ} \mathrm{C}$. Thus it was observed that conventional machined assembly specimens dissipated 20\% more heat and the direction of the temperature concentration and heat dissipation was $\pm 45^{\circ}$ which was the direction of failure of the CM specimens and it was also the direction of the fiber orientation.

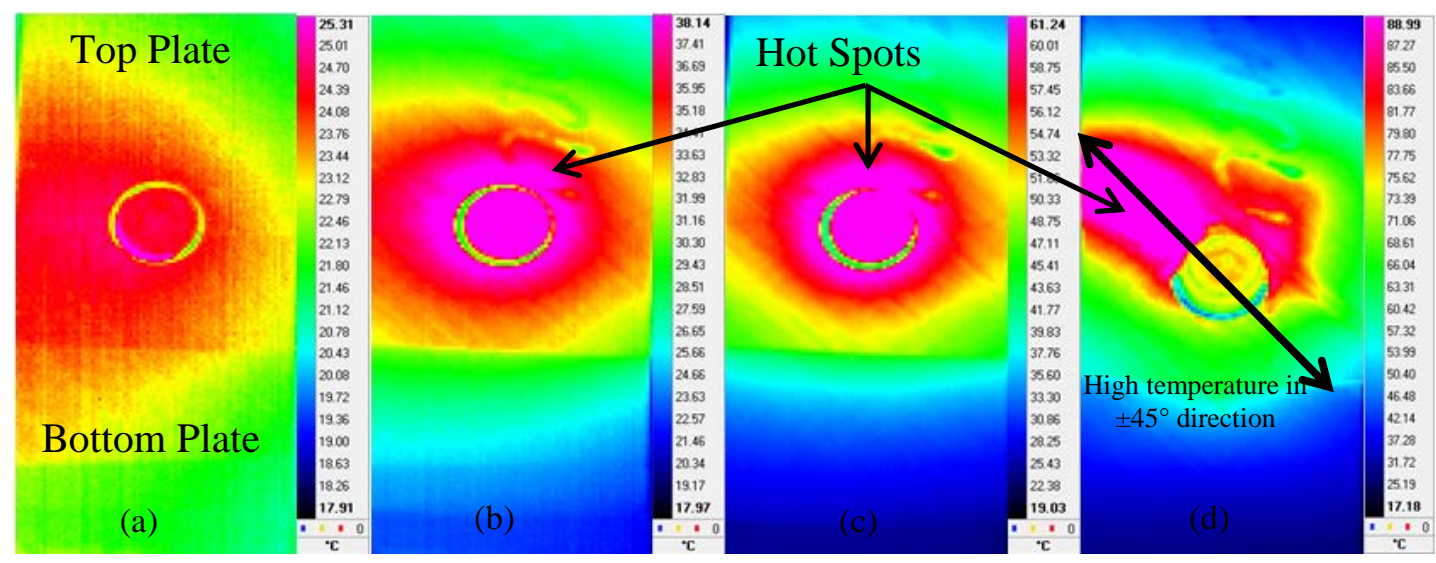

Figure 7-7 Temperature maps for loads of (a) $25 \%$, (b) $45 \%$ (c) $65 \%$ and (d) $80 \%$ of AWJM angle ply lay-up assembly specimen tested at $10 \mathrm{~Hz}$ frequency and 10000 cycles. 


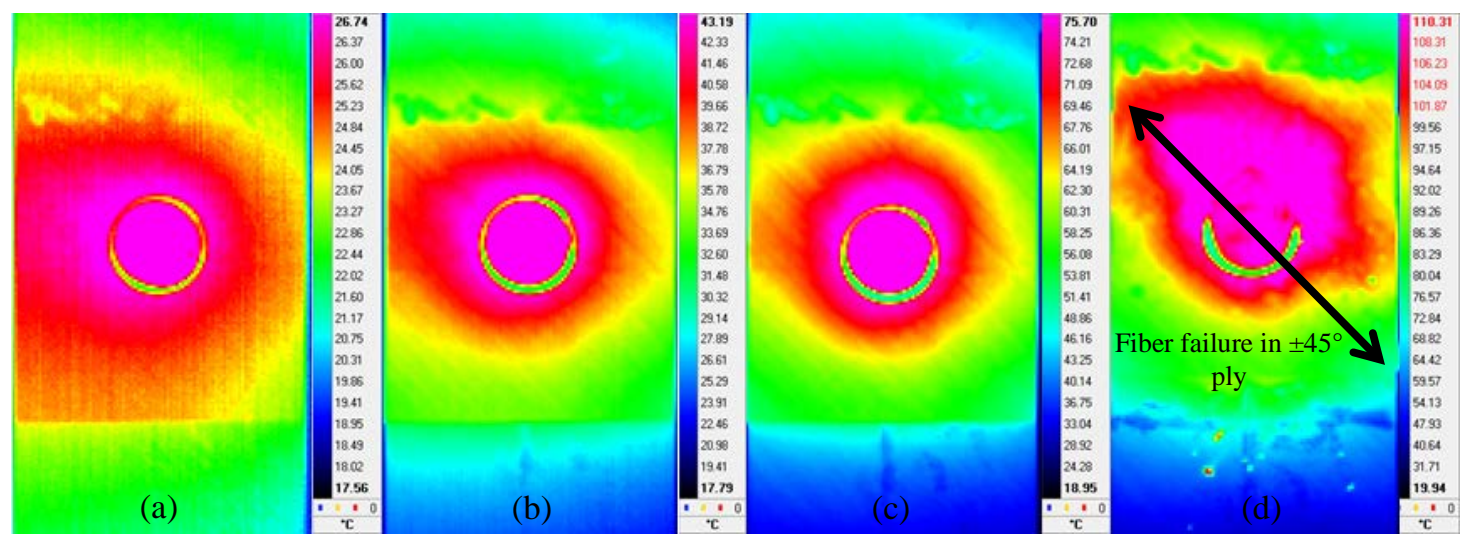

Figure 7-8 Temperature maps for loads of (a) 25\% (b) 45\% (c) 65\% and (d) 70\% for CM angle ply lay-up assembly specimen tested at $10 \mathrm{~Hz}$ frequency and 10000 cycles.

Temperature images illustrated in Figure 7-9 and Figure 7-10 show almost the same temperature $\left(25^{\circ} \mathrm{C}\right)$ initially recorded for CM and AWJM quasi isotropic lay-up assembly specimens. The temperature remained almost same $\left(36^{\circ} \mathrm{C}\right)$ up to $40 \%$ load of UTS for CM and AWJM assembly specimens. The temperature increased moderately $\left(49^{\circ} \mathrm{C}\right)$ till $65 \%$ load of UTS. Finally, an abrupt increase in temperature $\left(68^{\circ} \mathrm{C}\right)$ was noted in $\mathrm{CM}$ specimens and failure occurred in the load stage of 75\% of UTS, whereas the AWJM specimens failed at the load stage of $85 \%$ of UTS when temperature reached $57^{\circ} \mathrm{C}$.

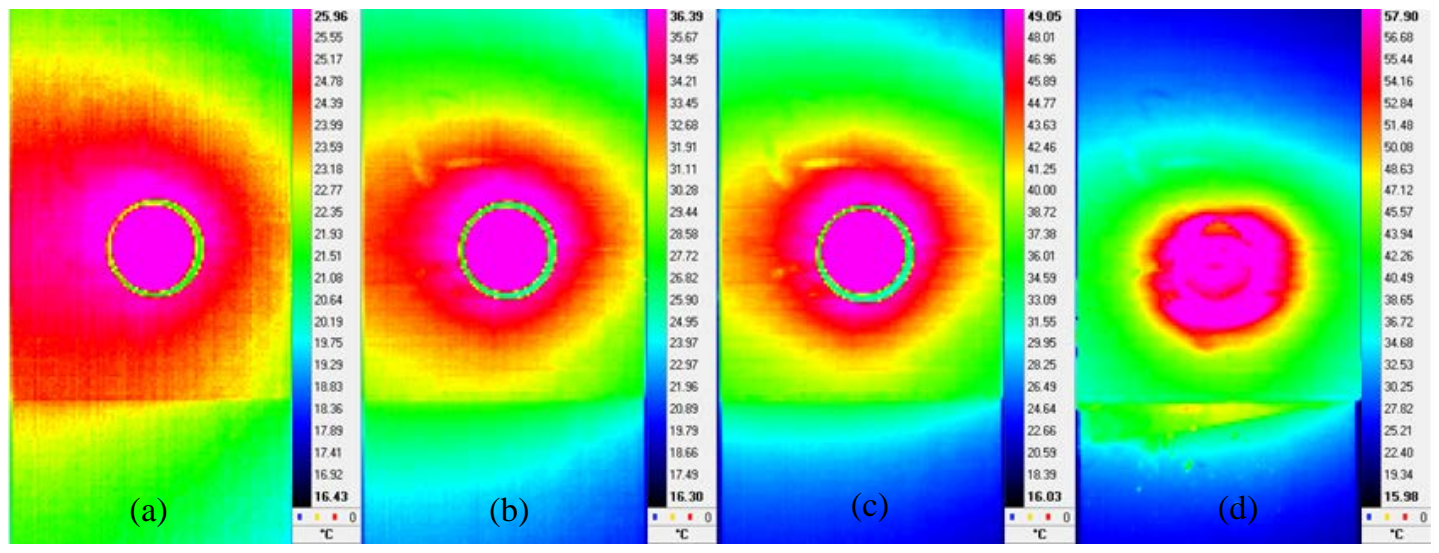

Figure 7-9 Temperature maps for loads of (a) 25\% (b) $45 \%$ (c) $65 \%$ and (d) $85 \%$ for AWJM quasi isotropic lay-up assembly specimen tested at $10 \mathrm{~Hz}$ frequency and 10000 cycles.

Figure 7-10d shows high heat concentrated and the beginning of the failure of CM specimens from $90^{\circ}$ plies. This failure is located at $-45^{\circ}$ (cf. Figure $7-10 \mathrm{~d}$ ). This showed that when 
conventional machining was used to make holes, the damage generated by the interaction between the cutting tool and the composite specimen was located in the vicinity of the plies orientated at $90^{\circ}$ and $-45^{\circ}$. Consequently, this conducts the early failure of CM specimens compared to the AWJM assembly specimens.

For both machining processes used, the increase in temperature was mainly related to the degradation of the matrix at a weak/damaged portion of the specimen induced by the machining process. The heat was also generated with the increase in friction between the bolt and the machined surface with the increase in cyclic load which contributed to the rise of temperature in CM and AWJM assembly specimens. Besides this, the heat generated by the friction between fibers/fibers and fiber/matrix was also responsible for heat dissipation. However, the results showed that streaks marks that resulted from the AWJM process generated less heat and damage during cyclic loading than fiber pull-out and matrix degradation areas, induced by the CM process. Hence the damage induced by the machining process was the main source of increase in temperature and damage.

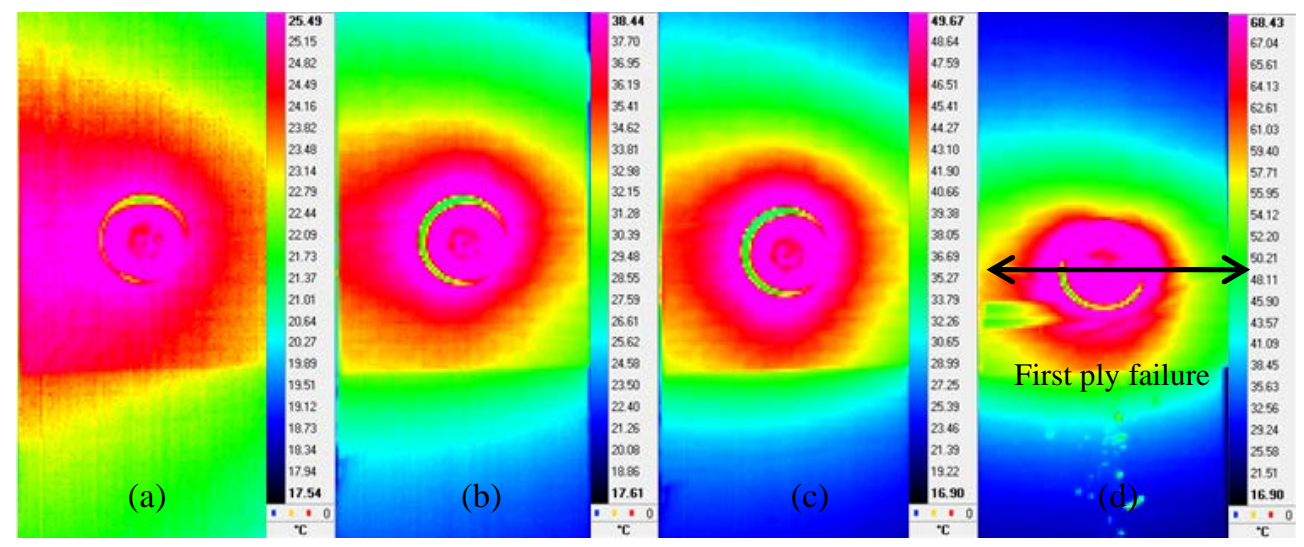

Figure 7-10 Temperature maps for loads of (a) 25\% (b) $45 \%$ (c) $65 \%$ and (d) $75 \%$ for CM quasi isotropic lay-up assembly specimen tested at $10 \mathrm{~Hz}$ frequency and 10000 cycles

From the infrared thermographic images (Figure 7-7 to Figure 7-10), it was observed that all CM assembly specimens dissipated more heat than AWJM assembly specimens. Furthermore, high heat dissipation was observed in assembly specimens having a stacking sequence of $\left[ \pm 45^{\circ}\right]_{2 \mathrm{~S}}$ than those having a [90/+45/0/-45]s stacking sequence. It is worth noting that, during infrared thermography tests, all specimens were inspected for any heat dissipation far from the machined hole in order to detect any defect created during the manufacturing of the composite laminates. 
However, for all specimens the temperature concentration, damage and failure were observed around the circular hole.

Figure 7-11 illustrates the correlation between the change in temperature and the damage accumulation against the normalized number of cycles in order to better understand the relationship between the mechanical damage and the heat dissipated. It is noticed that the increase in heat dissipation in various step loading corresponded to the damage evolution in the same step loading stages. The increase in temperature is related to matrix degradation, fiber pull-
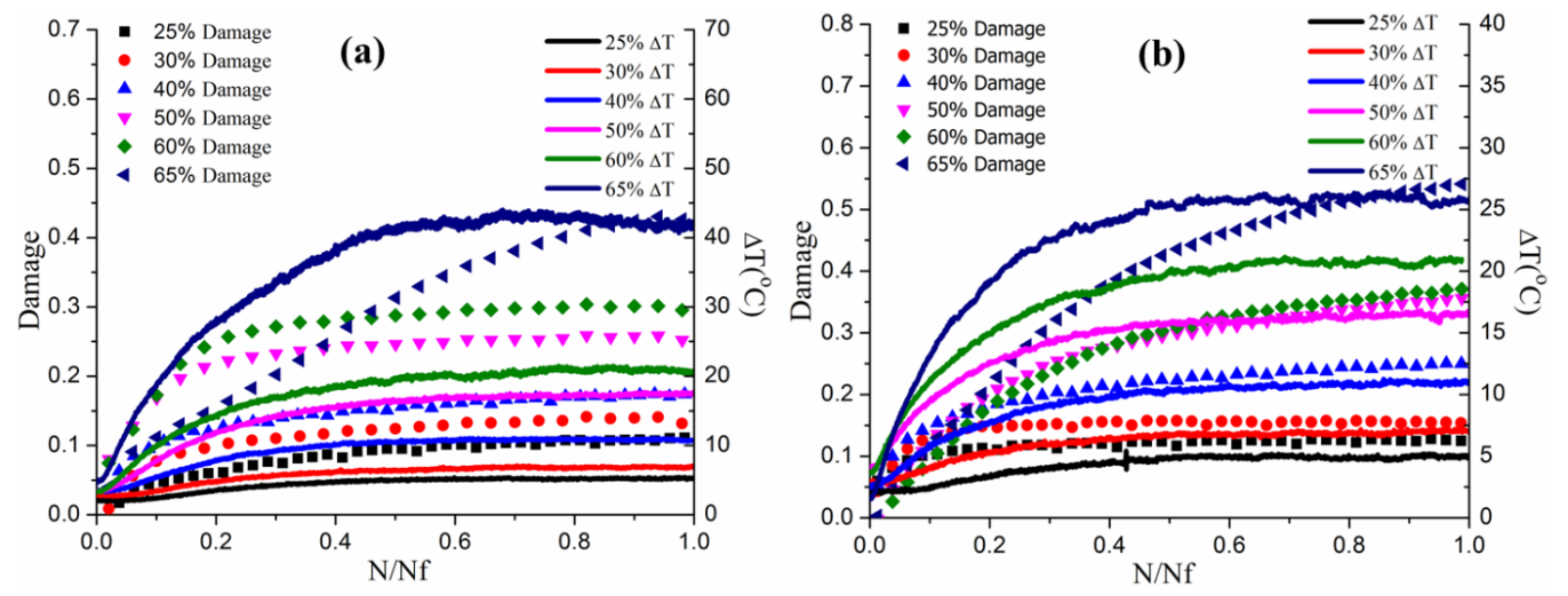

Figure 7-11 Graphs showing correlation of damage and temperature of (a) angle ply and (b) quasi isotropic lay-up assembly specimens drilled with AWJM process

out/breakage and the friction generated due to the contact pressure created between the bolt-hole surface during fatigue cyclic loading as explained above. Consequently, fiber/matrix degradation and the delamination phenomena take place, which resulted in the further increase of temperature as the number of loading cycles increased. This increase in temperature continued till the final failure of the assembly specimen. Hence, damage accumulation and temperature can be related to each other as shown in Figure 7-11. In this way we can also validate the results obtained from the extensometer (loss of stiffness) with the heat dissipation obtained from infrared thermography.

\subsection{High Cycle Fatigue Strength}

Using the infrared thermographic technique, high cycle fatigue strength (HCFS) can be obtained from the temperature curves by intersecting the two straight lines that interpolate the stabilization 
temperature $(\Delta T)$ and the corresponding stress level [150]. This can be obtained from the following equation;

$$
\Delta \mathrm{T}=\mathrm{T}_{\mathrm{f}}-\mathrm{T}_{0}
$$

where $\Delta \mathrm{T}$ is the change in temperature, $\mathrm{T}_{\mathrm{f}}$ is the final temperature and $\mathrm{T}_{0}$ is the initial temperature.

Using Equation (48), the change in temperature for all specimens was calculated for 10000 cycles for different stress levels. Figure 7-12 and Figure 7-13 shows HCFS plots for AWJM and
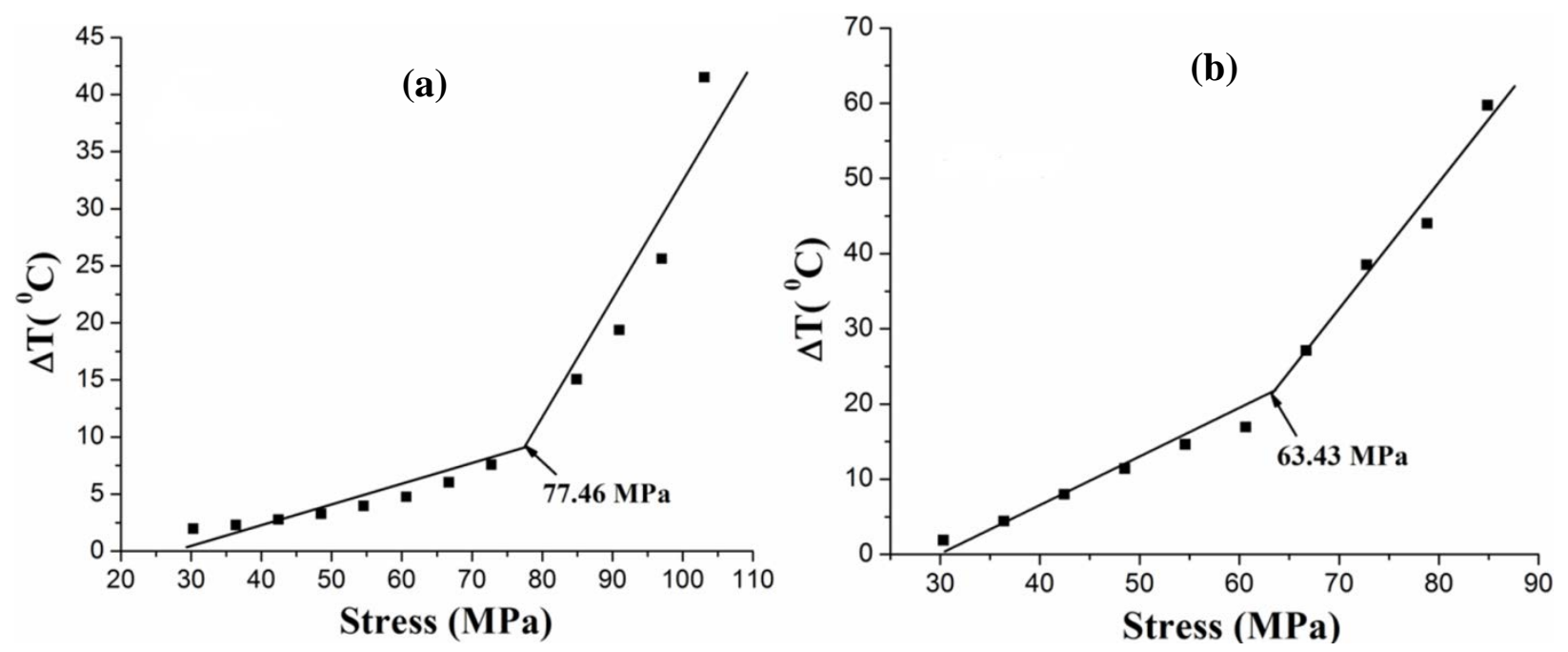

Figure 7-12 The high cycle fatigue strength of CF/Epoxy angle ply lay-up assembly specimens machined with (a) AWJM and (b) CM process, tested at 10Hz frequency at 10000 cycles.

CM assembly specimens for the stacking sequence of $\left[ \pm 45^{\circ}\right]_{2 S}$ and $[90 /+45 / 0 /-45]_{s}$. The HCFS calculated for the case of $\left[ \pm 45^{\circ}\right]_{2 S}$, drilled with AWJM process was $77.06 \pm 0.45 \mathrm{MPa}$, whereas for CM assembly specimen of the same stacking sequence, it was underestimated by $16.97 \%$ and its value was $63.98 \pm 0.57 \mathrm{MPa}$. For the case of the AWJM laminates with $[90 /+45 / 0 /-45]_{S}$ stacking sequence, 15.71\% improvement in HCFS was found from the laminates drilled with conventional tool of the same stacking sequence. In this case, the HCFS for CM and AWJM specimens was $65.74 \pm 0.26 \mathrm{MPa}$ and $77.99 \pm 2.43 \mathrm{MPa}$, respectively. In addition, these graphs also shows that the fatigue life is dependent on the $\Delta \mathrm{T}$ values i.e. the larger the $\Delta \mathrm{T}$ value, the shorter the fatigue life. The higher improvement in the HCFS of AWJM assemblies was associated with the surface quality generated during the machining processes, which was already 
discussed in section 7.1. From these results it is clear that, the high cycle fatigue strength of the drilled specimens made of composite materials dedicated to the assembling, cannot be characterized by the roughness criteria $(R a)$ initially developed for the isotropic materials.
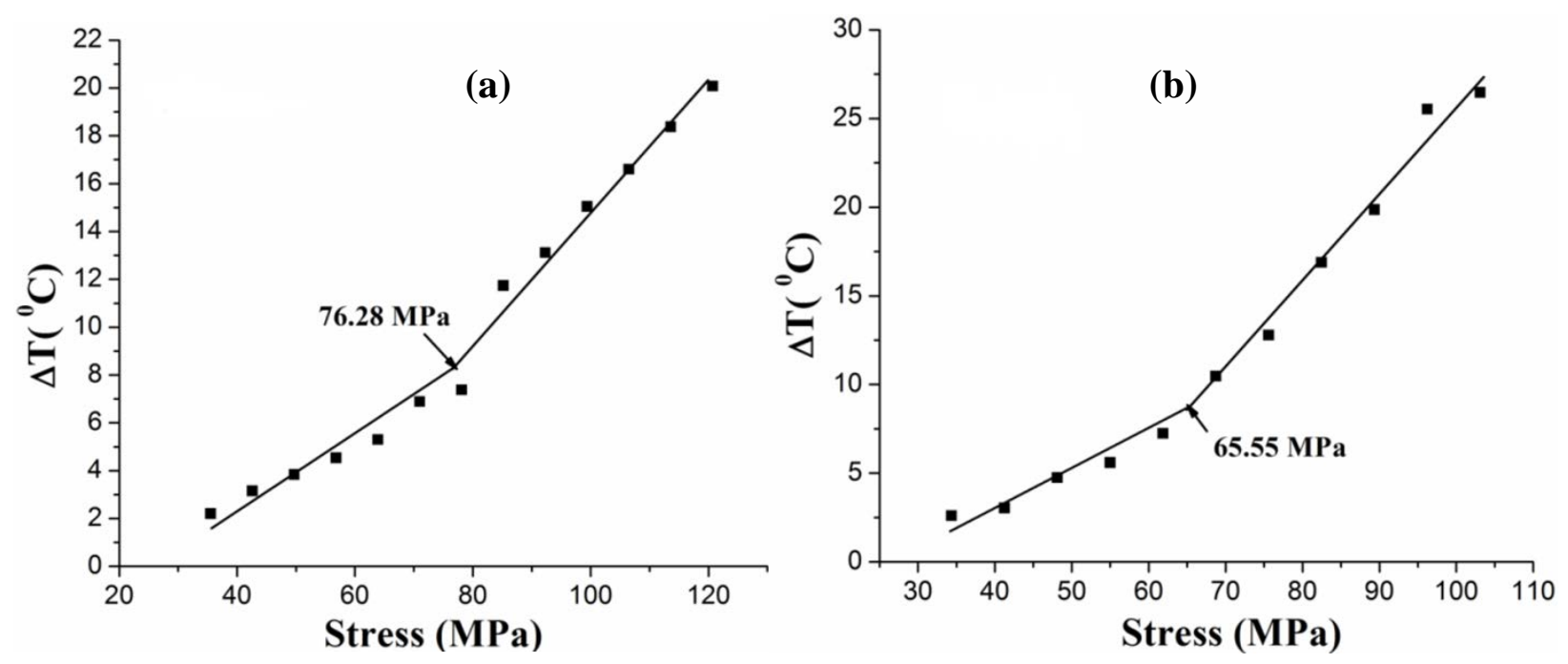

Figure 7-13 The high cycle fatigue strength of CF/Epoxy quasi isotropic lay-up assembly specimens machined with (a) AWJM and (b) CM technique, tested at $10 \mathrm{~Hz}$ frequency at 10000 cycles.

\subsection{Fatigue failure analysis}

In order to investigate the failure mechanism of single-lap, single-bolted joint assembly specimens, IR images and a post-mortem analysis of the photographs of the fractured specimens were analyzed. All specimens failed around the joints, therefore this area was considered for the failure mechanism examination. Figure 7-14 and Figure 7-15 depicts IR images of the failure of CF/Epoxy composite assembly having lay-ups of $\left[ \pm 45^{\circ}\right]_{2 S}$ and $[90 /+45 / 0 /-45]_{s}$. Figure 7-14 (a, b) shows the failure of the AWJM assembly specimen of angle ply lay-up, which shows fastener pull-through failure mode as a result of the delamination of plies along the loading direction. However, for AWJM assembly specimens, I think that the streak marks illustrated in Figure 7-4b can not be responsible for crack initation and then propagation. Two main mechanisms were responsible for failure of AWJM $\left[ \pm 45^{\circ}\right]_{2 s}$ assembly specimen. Firstly, the intense friction created between bolt and the machined surface was mainly responsible for the crack initiation in the matrix of these specimens during initial step loading stages as explained above. This also increased the stress concentration around the hole surface with the increase in cyclic load, which 

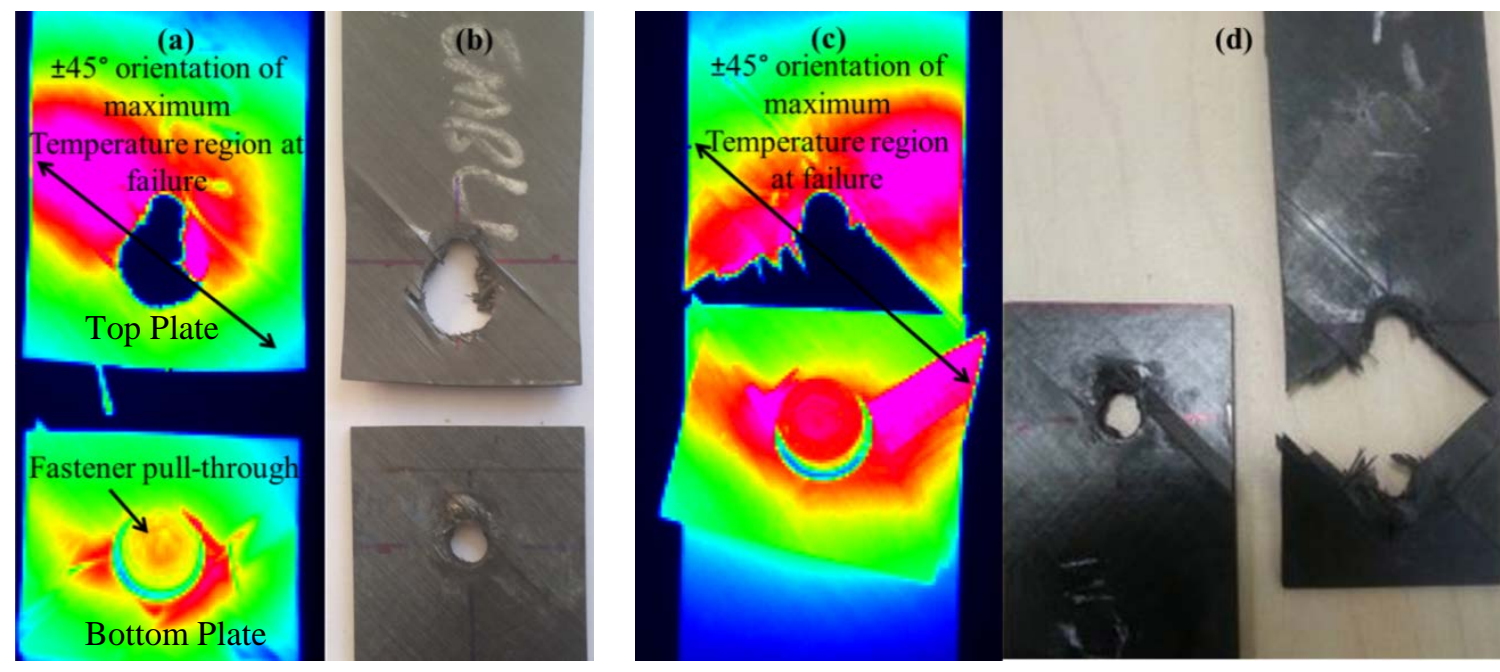

Figure 7-14 Images showing failure mode of CF/Epoxy angle ply lay-up laminates (a) IR image and (b) photograph of AWJM fractured specimen. While (c) and (d) shows IR image and photograph of CM fractured specimen, respectively

resulted in the damage of the fiber and matrix around the hole. Secondly, due to the cyclic loading, interlaminar frictional shear forces were developed more and more between fibers/fibers and/or fibers/matrix interface, resulting in crack propagation which consequently created damages. With the further increase in the loading cycles, the frictional forces further created damages in the fiber and matrix, and were also responsible for the elongation of the circular hole (Figure 7-5 and Figure 7-6). These damages also resulted a thermal gradient shown on the surface of the assembly specimen with the hot spots (Figure 7-7) in the region of the maximum stress conentration where the said damages were created. These damaged fibers and matrix reduced the strength around the hole surface and caused bolt tilting with the applied tensile load, hence creating clearance between the hole and bolt surface with the increase in the number of loading cycles. Tilting the bolt further deformed the surface of the hole due to the intense contact pressure. This tilting reduced the strength of AWJM angle ply lay-up assembly specimens which led to the final failure. The failure of assembly specimens with a stacking sequence of $\left[ \pm 45^{\circ}\right]_{2 S}$ and machined with the AWJM process occurred by fastener pull-through (bearing failure) mode at $80 \%$ load of UTS $(5.74 \mathrm{kN})$.

Concerning the CM assembly specimens of angle ply lay-up (Figure 7-14b), the specimen failure was observed along the fiber direction, i.e. along a $\pm 45^{\circ}$ direction. The initial damages i.e. the pull-out of fibers and matrix degradation were created during the CM (cf. Figure 7-4a). These 
pulled out fibers and the degraded matrix acted as nucleation points for fatigue cracks, which further propagated in CM assembly specimens with the increase in loading cycles and consequently reduced the strength of the specimen which was followed by an abrupt increase in damage as the number of loading cycles further increased and finally resulted in the catastropic fracture of the assembly along the fiber direction i.e. $\pm 45^{\circ}$. The failure modes for CM specimens were the bearing of one lap and the shear out of the other lap (Figure 7-14b).

The failure of quasi isotropic lay-up assembly specimens machined with the AWJM process is shown in Figure 7-15 (a1 to e1). In this case failure occurred in the fastener pull-through mode
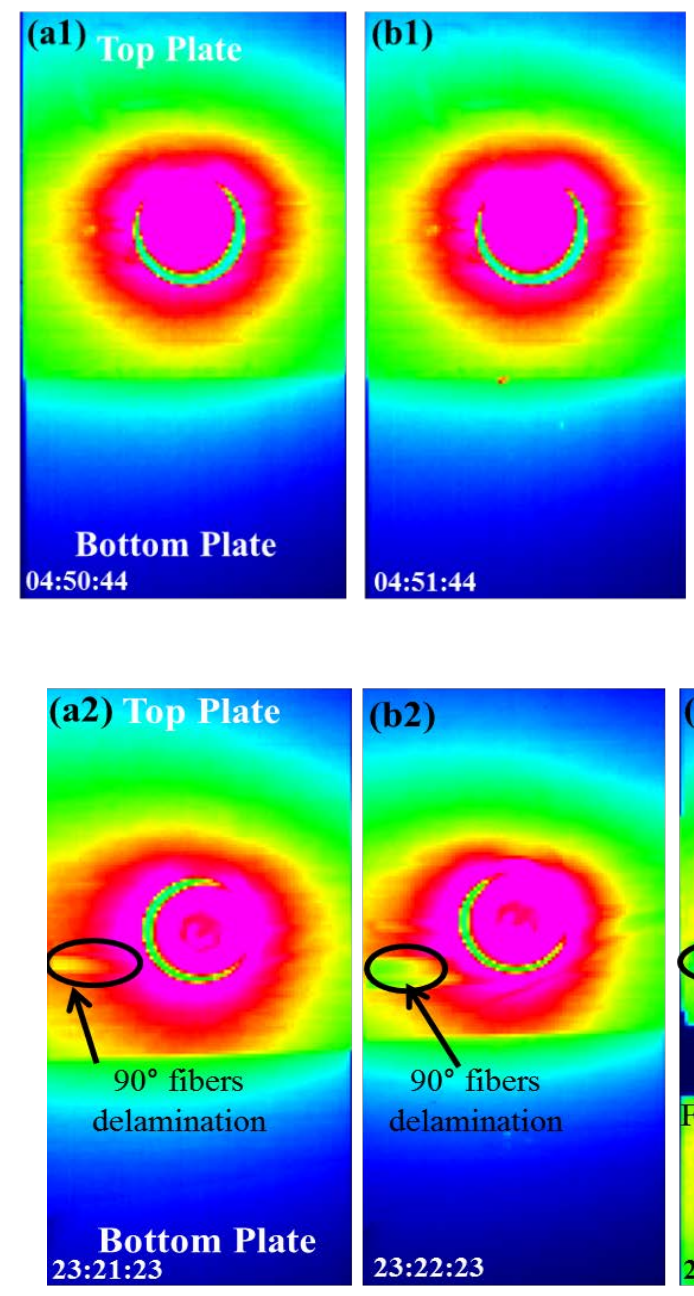
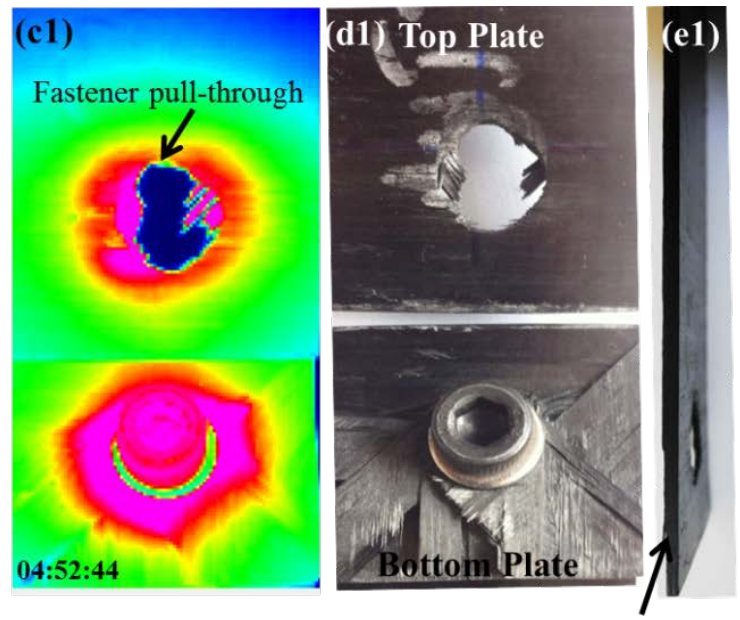

No mid-plane delamination

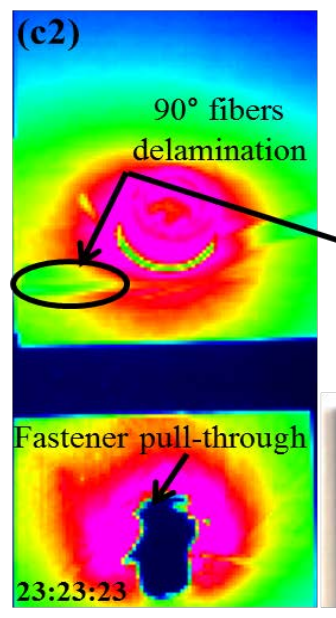

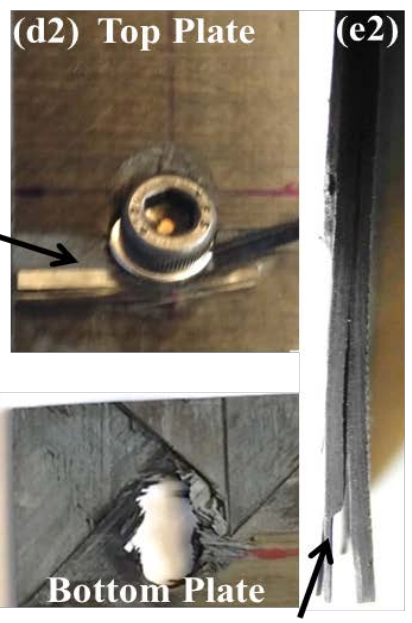

Mid-Plane delamination

Figure 7-15 Images showing failure modes of CF/Epoxy quasi isotropic lay-up composite assembly. IR images (a1,b1) and (a2,b2) shows images before final failure, IR images (c1) and (c2) shows failure, while (d1) and (d2) is the photograph of fractured specimen and (e1) and e2 illustrates fractured specimens from thickness side of AWJM and CM laminates, respectively 
and followed the same damage mechanism which was found in angle ply composite assembly specimens drilled with AWJM process.

The failure mechanism of CM specimen with quasi isotropic lay-up is shown in Figure 7-15 (a2 to e2). Similarly to quasi isotropic lay-up AWJM specimens, the failure in CM specimens also occurred due to fastener pull-through mode, however the failure modes were different. At first, delamination induced by the matrix cracking started in the matrix dominated ply ( $90^{\circ}$ ply) (Figure 7-15a2) which further propagated with the increase of cyclic load (Figure 7-15b2). It is worth mentioning here that the appearance of delamination of $90^{\circ}$ ply can also be related to the initial damages (fiber pull-out and matrix degradation) produced by the conventional machining process (cf. Figure 7-4a). This delamination in the $90^{\circ}$ ply around the circular hole decreased the strength of the CM assembly specimens. Furthermore, the mid-plane delamination, which can clearly be observed from Figure 7-15e2, was also responsible for the final failure. This type of mid-plane delamination can not be seen in the specimens machined with the AWJM process (Figure 7-15e1). This mid-plane delamination of $-45^{\circ}$ ply in case of CM assembly specimens, was mainly due to the surface quality generated by the machining process (pull-out of $-45^{\circ}$ fibers and matrix degradation) which corresponds to the same location of the damage induced by the machining process as identified in surface roughness images (Figure 7-4a). The same behaviour was also stated in [151] which showed that due to the non-homogeneous behaviour of composite laminates, a roughness defect was observed on the drilled hole which was due to the fiber direction in relation to the direction of the rotation of the cutting tool. The complete shearing of $+45^{\circ}$ and tearing of $-45^{\circ}$ fibers was investigated by [151].

In addition to the fiber pull-out $\left(-45^{\circ}\right)$, the strength of the CM assembly specimen around the circular hole was also affected by stress concentration created, owing to the application of a steel bolt used for joining purpose. This consequently decreased the strength of the machined surface which resulted in the abrupt failure of the quasi isotropic assembly specimen drilled with the CM process. Failure in this case occurred in the fastener pull-through mode. The delamination of the last ply (matrix dominated ply) was also observed during the examination of the fractured specimen, which was due to the fastener pulled out from the hole at the time of failure and can be seen in Figure 7-15d1 and Figure 7-15d2. 


\subsection{Summary}

The current thesis investigated the mechanical behaviour of CF/Epoxy composite bolted joint assembly specimens drilled with CM and AWJM processes. Two stacking sequences namely; angle ply $\left(\left[ \pm 45^{\circ}\right]_{2 S}\right)$ and quasi isotropic $\left([90 /+45 / 0 /-45]_{S}\right)$ were tested. This is the first study that uses destructive and non-destructive techniques (including fatigue testing, IR thermography, surface roughness and SEM measurements) to identify the effect of surface quality (texture) on the mechanical performance of CFRP laminates. The following conclusions were drawn from the present investigation:

- Conventional machined specimens accumulated 15\% more damage than specimens drilled with AWJM. This difference in the mechanical behaviour exhibited by the assembly specimens was attributed to the surface quality generated by each machining process. It appears from the results of the CM specimens of both lay-ups, that $-45^{\circ}$ plies were more susceptible to damage and delamination during fatigue tests. Therefore AWJM is more suitable for composites of all stacking sequences than the conventional machining process.

- The composite assembly specimen drilled with the AWJM process shows more than 15\% higher HCFS than those drilled with the CM process. Thus it is highly recommended that for critical structural assembly, the AWJM process should be taken into account.

- The joining of the composite laminates with the fastener reduces the mechanical properties during cyclic loading when compared to a single plate specimen with a circular hole.

- Heat dissipation from the machined surface can be used as a suitable criterion to predict the propagation of crack and finally the damage assessment in the composite material structural assembly.

- This study concluded that the CM is inferior to the AWJM process in terms of the quality of holes and failure life. Hence the failure mode and the mechanical behaviour of all laminates tested were affected by the type of the machining processes used for the manufacturing of circular holes in composite laminates.

All of the above observations showed that the machining process plays an important role on the mechanical behaviour of CF/Epoxy composite laminates. 


\section{CHAPTER 8. CONCLUSION, CONTRIBUTIONS AND FUTURE WORK}

\subsection{Conclusions}

CF/Epoxy structural component and single-lap, single-bolted joint assembly specimens with different lay-ups namely, angle ply and quasi isotropic, were investigated after drilling with conventional machining (CM) and abrasive water-jet machining (AWJM) processes. These specimens were subsequently put to static and fatigue tensile tests to evaluate the influence of the hole surface defects on the mechanical performance. The most distinguished observations that can be concluded from present study are briefly presented here:

- The static tensile testing did not indicate any significant difference in the mechanical response between CM and AWJM specimens (i.e. strength and stiffness), as both specimen types had similar ultimate tensile strengths and strains. Hence, composite material is less sensitive to the machining process in the area where static load is applied to the machined part or assembly.

- The surface roughness images of pre-fatigue laminates indicated that the mechanical behaviour and failure modes of composite laminates are highly affected by the choice of the machining process as well as the type of stacking sequence. It appears from the results of the $\mathrm{CM}$ specimens that $-45^{\circ}$ plies are more susceptible to damage and delamination during fatigue tests. Fiber pull-out from $-45^{\circ}$ plies are related to material removal mechanisms which are strongly influenced by the relative angle between the direction of the cutting speed and the direction of the fibers. The surface roughness parameter $(R a)$ indicated that the AWJM and CM surface have the same roughness values, although the type and the form of the damage generated by each machining process are completely different. This was confirmed by SEM tests conducted after a destructive sectioning of the pre-fatigue specimens. Crack density was also calculated to investigate the damage induced by two machining processes. Crack density further confirmed the roughness and SEM results.

- All single rectangular plate specimens have shown similar damage accumulation for lesser loads. For higher loads, the damage is more significant for the specimens of different stacking sequences drilled using conventional machining than the AWJM specimens. The effect of the damage induced (fiber pull-out and matrix degradation) by 
the machining technique was evident from high damage accumulation in all laminates drilled with the conventional machining process during fatigue tests. SEM observations of the post-fatigue specimens confirm that the fiber pull-out and matrix degradation areas on the surface of CM specimens act as a stress concentrator for crack initiation and propagation. In comparison, in the case of striation marks and craters defects induced by the AWJM process on the machined surface, it was susceptible to less damage during fatigue tests. Furthermore, the effect of test parameters were correspondingly compared and found that increasing tests parameters decreases the fatigue life. However, the failure mode and the mechanical behaviour of all laminates were affected by the choice of the machining process and the stacking sequence used for the manufacturing of composite laminates.

- Conventional machined assembly specimens accumulated 15\% more damage than singlelap, single-bolt joint assembly specimens drilled with AWJM. This difference in the mechanical behaviour exhibited by the assembly specimens was attributed to the surface quality generated by each machining process.

- Infrared thermographic images were analyzed for thermal dissipation; the maximum temperature profiles in the area surrounding the hole (i.e., studied area) followed the same evolution as the damage profiles for the laminates drilled with both machining processes. However, the presence of damages induced by the cutting tool in conventional machining provoke more heat dissipation during fatigue testing than the ridges and craters damages observed on the wall of the holes machined by the abrasive water-jet process. It was found that, when conventional machining was used, the damage and heat dissipation in the angle ply laminates were more pronounced than that found in quasi isotropic ones. Furthermore, quasi isotropic laminates showed a linear mechanical response during fatigue loading and the type of fracture observed was brittle as compared to the non-linear mechanical response of angle ply laminates due to the progressive damages. Therefore, angle ply laminates, due to their non-linear mechanical response, dissipated more heat than quasi isotropic laminates. However, when comparing machining processes, AWJM dissipated less heat during fatigue loading than the CM specimens of all tested laminates. These results corresponded to the damage accumulation in these laminates. Hence, the AWJM process can efficiently be used for 
making holes in all types of composite laminates and also can effectively work under a fatigue loading condition compare to CM specimens.

- IR thermography can be effectively and successfully utilized to identify a suitable machining process for aerospace grade CFRP composite assembly and its parts during fatigue testing. Furthermore, IR thermography can efficiently be used for the in-service monitoring of crack initiation and propagation. Heat dissipation from the machined surface can be used as a suitable criterion to predict the propagation of crack and damage assessment in composite laminates.

- The endurance limit for the structural part and assembly specimens drilled with CM was 10-17\% inferior compared to the AWJM specimens of different stacking sequence. This improvement in endurance limit for specimens drilled with AWJM process can be related to the initial surface integrity after machining induced by the difference in the mechanism of material removal between the two processes used.

- IR thermal concentration factor was calculated which was developed from the infrared thermographic stress equation and was compared to the stress concentration factor. IR thermal and stress concentration values of the CM specimens were over-estimated by $15.70 \%$ and $16.30 \%$ compared to AWJM specimens. The Point Stress Criterion (PSC) and the Average Stress Criterion (ASC) proposed by Tercan and Whitney were also calculated and compared with the concentration factor derived from the IR thermal and stress analysis to investigate whether the type of machining affects these stress criteria or not. The stress concentration values for CM specimens were $14.85 \%$ and $18.35 \%$ greater than the values calculated from the point and average stress criterion, respectively. In the case of the AWJM specimens, the stress concentration values were close to the analytically calculated values obtained from PSC (1.95) and ASC (1.87) which showed that striation marks created in AWJM process produced less stress concentration sites. It can clearly be seen that the effect of the machining process on the thermal and stress concentration was more pronounced in the CM specimen compared to the AWJM specimen and consequently this reduced the mechanical properties of the CM specimens, therefore, the CM specimens failed earlier than AWJM specimens.

- A Thermographic Damage Criterion (TDC) based on heat dissipation was developed in order to assess the effect of two types of machining processes (conventional machining 
vs. abrasive water-jet machining) on the mechanical behaviour of CFRP specimens. For all applied loads, the TDC of specimens machined with AWJM was lower than the TDC of specimens drilled with CM. TDC further confirms the difference between the specimens drilled with AWJM and CM processes. These results were in good agreement to that observed with the loss of rigidity obtained from the extensometer. However, regardless of the machining process used and for the same load, the damage rates calculated from the heat dissipation (TDC) are larger than those calculated from the decrease of rigidity.

- Angle ply assembly specimens drilled with CM failed along the fiber direction. These failure modes were consistent with the surface roughness images. However, for the quasi isotropic assembly specimens, the failure mode was a combination of fastener pullthrough and mid-plane delamination. Unlike CM, no mid-plane delamination was observed in the case of AWJM quasi isotropic assembly specimens. Mid-plane delamination in CM quasi isotropic assembly specimen was due to the defect induced by the machining process in $-45^{\circ}$ ply. The failure mode of the assembly specimens drilled with AWJM was fastener pull-through dominated for both studied stacking sequences. This indicates that the AWJM process is less sensitive to the composite's stacking sequence compared to conventional machining.

- The roughness criteria generally used to quantify the quality of machined surfaces are not representative of the mechanical performances of all the tested specimens. In addition, they cannot be the representative of the subsurface damage. One can conclude that unlike metallic materials, the criterion used for quantifying the quality of machining based on the average roughness $(R a)$ is not suitable for composite materials.

Finally, all observations of this study indicated that the choice of the machining process has a significant impact on the mechanical behaviour and functionality of CRFP structure parts and assembly. 


\subsection{Contributions}

The main contributions made through this research are summarized in the following and that were published/under review and presented in related journals, book section and conferences $[111,152-156]$;

- This research is the first to study the effect of the surface texture obtained by two different machining processes (namely, abrasive water-jet vs conventional machining) on the mechanical behaviour of CFRP composites. Previous works focused only on conventional axial or orbital drilling, furthermore, a comparative study of both machining processes for the effect of the surface quality of a structural part is not yet investigated. There is also no research work available in open literature that correlates the fatigue behaviour of the assembly specimens drilled with abrasive water-jet machining process to the surface integrity. To the best of my knowledge, this is the first study that investigated the influence of the CM and AWJM processes on the mechanical behaviour of the aerospace grade composite structure part and assembly and correlated the mechanical behaviour to the machined surface quality. The mechanical behaviour of these specimens was investigated using different analytical and experimental techniques. The results of this research will also be helpful for the manufacturing engineers to select an appropriate machining process for the areas where defect free structural component is required.

- From literature review it was investigated that the criterion used for metallic materials to quantify the quality of machined surface based on the average roughness $(R a)$ affects the fatigue life. It was observed that these criteria cannot describe the subsurface flaws/cracks. In current research it was investigated that these roughness parameters have no contribution towards the fatigue life of the composite laminates drilled with two different types of machining processes using particular combination of the drilling parameters. As $R a$ for CM and AWJM specimens was same, however different mechanical behaviour of the two types of the laminates was observed. The main contributing factor towards this difference was the damages induced by the machining process, which is another significant contribution of the present research.

- The development of the thermal concentration factor to identify influence of the machining process on mechanical behaviour by utilizing infrared thermographic concept 
is again a unique and another significant contribution to the present research. Furthermore, the concentration factors derived from the IR thermal and stress analyses were confirmed with the point stress criterion (PSC) and the average stress criterion (ASC) to investigate whether the type of machining affects these stress criteria or not. These stress criteria have not yet been studied for comparing two machining processes for its suitability.

- The development of the thermographic damage criteria is another unique contribution to this research. This criterion predicts the damage evolution by considering the increase in the thermal threshold. TDC confirmed that, during fatigue testing heat dissipation from the machined surface can be used as a suitable criterion to predict the propagation of a crack and finally the damage assessment in the composite laminates. Hence the heat dissipation can be correlated with the damage accumulation. Thus this criterion can be used for the suitability of different machining processes, which is a unique contribution of this study.

- A detailed study of the static and fatigue behaviour of the laminates drilled with a conventional and non-conventional machining process was done, in which the machined surface correlated with the mechanical properties during fatigue tests. The machined surface which caused the degradation of the engineering components that finally led to the failure, was identified. It was identified that the AWJM process can efficiently be used for machining holes in all types of composite laminates and can also effectively work under fatigue loading conditions compare to CM laminates.

- This is the first study that uses an infrared thermographic technique to monitor the actual step by step heat dissipation by the drilled surface generated from two machining processes. IR thermography can be effectively utilized to access the mechanical performance of CFRP composite structural part and assembly to find a suitable machining process, which has not been previously employed for such a study.

- In the present research, the experimental work was performed with a combination of techniques to monitor damage detection. We used local and global damage detection techniques. For local damage detection, an extensometer was used and an infrared thermographic technique was utilized as a global damage detection technique. A 
combination of these techniques has not been previously employed for the damage assessment of the machined surface of aerospace grade composite structure parts and assembly.

- A list of publications and manuscripts is currently in progress, related to the current research is presented in Appendix A-13.

\subsection{Future work}

Though this research gave a clear idea of the effect of the machining process on the mechanical behaviour of CFRP composite laminates, more research can be done to further explore the effect of machining processes on the aerospace grade composite laminates. A summary of recommendations is given below.

- Future studies may wish to develop an FE model to investigate and validate the effect of the surface quality on the damage accumulation and the fatigue life of CFRP composite laminates.

- It is recommended to investigate the effect of the surface quality on the mechanical behaviour, especially the damage, under other modes of loading such as bending, torsion and compression, and identify whether the mode of loading has a significant impact on the effect of the surface quality

- Futur work may also include fatigue tests on CFRP composites with various hole diamaters to evaluate the effect of the hole diameter on the mechanical behaviour of the composite.

- The current research investigated only two configurations (i.e., anply ply and quasi isotropic). Other ply configurations should be examined.

- Finally, a future study could involve investigating the possible effects of the composite's material on the surface quality. 


\section{Appendices}

\section{Appendix A-1 Matlab code for calculating Material Properties and Stresses}

\% This M-file calculates stiffness and compliance matrices as well as A, B

$\%$ and $\mathrm{D}$ matrices for an arbitrary laminate.

epsilon11G=input('Enter epsilon11G:');

epsilon21G=input('Enter epsilon21G:');

epsilon31G=input('Enter epsilon31G:');

epsilonG=zeros(3,1);

epsilonG=[epsilon $11 \mathrm{G}$

epsilon21G

epsilon31G];

sigmaL=zeros $(3,1)$;

$\%$ Enter the Young's modulus of fiber and matrix

$\mathrm{Ef}=$ input('Enter the Young modulus of the fiber ( $\mathrm{Ef}$ in GPa):');

Em=input('Enter the Young modulus of the matrix (Em in GPa):');

$\%$ Enter the Poisson's ratio of fiber and matrix

Nuf=input('Enter the Poisson ratio of the fiber (Nuf):');

Num=input('Enter the Poisson ratio of the matrix (Num):');

\% Enter the volume fraction of fibers within each lamina

$\mathrm{Vf}=$ input('Enter the volume fraction of fibers within each lamina $(\mathrm{Vf}<1)$ :');

$\mathrm{Vm}=1-\mathrm{Vf}$

\% Calculation of longitudinal and transversal Young's modulus of the lamina

disp('Longitudinal and Transversal Youngs modulus of the lamina (in GPa), respectively:')

$\mathrm{EL}=\mathrm{Ef} * \mathrm{Vf}+\mathrm{Em} * \mathrm{Vm}$

$\mathrm{ET}=\mathrm{Ef} * \mathrm{Em} /(\mathrm{Ef} * \mathrm{Vm}+\mathrm{Em} * \mathrm{Vf})$

$\%$ Calculation of Poisson's ratio of the lamina

disp('Poisson ratios of the lamina:')

$\mathrm{NuLT}=\mathrm{Nuf} * \mathrm{Vf}+\mathrm{Num} * \mathrm{Vm}$

$\mathrm{NuTL}=(\mathrm{ET} / \mathrm{EL}) * \mathrm{NuLT}$

$\%$ Calculation of shear modulus of the fiber and matrix

disp('Shear modulus of the fiber and matrix (in GPa), respectively:') 
$\mathrm{Gf}=\mathrm{Ef} /(2 *(1+\mathrm{Nuf}))$

$\mathrm{Gm}=\mathrm{Em} /(2 *(1+\mathrm{Num}))$

$\%$ Calculation of transverse shear modulus of the lamina

disp('Transverse shear modulus of the lamina (in GPa):')

$\mathrm{GLT}=\mathrm{Gf} * \mathrm{Gm} /(\mathrm{G} f * \mathrm{Vm}+\mathrm{Gm} * \mathrm{Vf})$

$\%$ Calculation of stiffness and compliance matrices for local coordinate system (L-T)

$\mathrm{Q} 11=\mathrm{EL} /(1-\mathrm{NuLT} * \mathrm{NuTL})$;

Q22=ET/(1-NuLT*NuTL);

$\mathrm{Q} 12=\mathrm{NuLT} * \mathrm{ET} /\left(1-\mathrm{NuLT}^{*} \mathrm{NuTL}\right)$;

$\mathrm{Q} 66=\mathrm{GLT}$;

disp('Stiffness matrix for local coordinate system (L-T):')

$\mathrm{Q}=[\mathrm{Q} 11 \mathrm{Q} 120$

Q12 Q22 0

00 Q66]

$\mathrm{S} 11=1 / \mathrm{EL}$;

$\mathrm{S} 22=1 / \mathrm{ET}$;

S12=-NuLT/EL;

S66=1/GLT;

disp('Compliance matrix for local coordinate system (L-T):')

$\mathrm{S}=[\mathrm{S} 11 \mathrm{~S} 120$

S12 S22 0

00 S66]

$\%$ Value of $\mathrm{n}$

$\mathrm{n}=$ input ('Enter the value of $\mathrm{n}:$ ');

$\mathrm{h}=\mathrm{zeros}(\mathrm{n}+1,1)$;

Theta=zeros(n,1);

$\mathrm{A}=\mathrm{zeros}(3,3)$;

$\mathrm{B}=\mathrm{zeros}(3,3)$;

$\mathrm{D}=$ zeros $(3,3)$;

for $\mathrm{i}=1: \mathrm{n}+1$

$h(i)=$ input ('Enter the appropriate values of h, from h0 to hn (in mm), respectively:'); 
end

thickness $=2.22$;

for $k=1: n$

\% Angle of Theta (Angle between loading and fiber directions)

Theta(k)=input('Enter the Angle between loading and fiber directions in each lamina, (Start from the top, Theta in Radian):');

\% Calculation of Transformation matrix

$\mathrm{T} 1=\left[\cos (\operatorname{Theta}(\mathrm{k}))^{\wedge} 2 \sin (\operatorname{Theta}(\mathrm{k}))^{\wedge} 22^{*} \cos (\operatorname{Theta}(\mathrm{k}))^{*} \sin (\right.$ Theta(k))

$\sin (\operatorname{Theta}(\mathrm{k}))^{\wedge} 2 \cos (\operatorname{Theta}(\mathrm{k}))^{\wedge} 2-2 * \cos (\operatorname{Theta}(\mathrm{k}))^{*} \sin (\operatorname{Theta}(\mathrm{k}))$

- $\left.\cos (\operatorname{Theta}(\mathrm{k}))^{*} \sin (\operatorname{Theta}(\mathrm{k})) \cos (\operatorname{Theta}(\mathrm{k}))^{*} \sin (\operatorname{Theta}(\mathrm{k})) \cos (\operatorname{Theta}(\mathrm{k}))^{\wedge} 2-\sin (\operatorname{Theta}(\mathrm{k}))^{\wedge} 2\right]$;

T1_1=inv(T1);

$\mathrm{T} 2=\left[\cos (\operatorname{Theta}(\mathrm{k}))^{\wedge} 2 \sin (\operatorname{Theta}(\mathrm{k}))^{\wedge} 2 \cos (\operatorname{Theta}(\mathrm{k}))^{*} \sin (\right.$ Theta $(\mathrm{k}))$

$\sin (\operatorname{Theta}(\mathrm{k}))^{\wedge} 2 \cos (\operatorname{Theta}(\mathrm{k}))^{\wedge} 2-\cos (\operatorname{Theta}(\mathrm{k}))^{*} \sin (\operatorname{Theta}(\mathrm{k}))$

$\left.-2 * \cos (\operatorname{Theta}(\mathrm{k})) * \sin (\operatorname{Theta}(\mathrm{k})) 2 * \cos (\operatorname{Theta}(\mathrm{k}))^{*} \sin (\operatorname{Theta}(\mathrm{k})) \cos (\operatorname{Theta}(\mathrm{k}))^{\wedge} 2-\sin (\operatorname{Theta}(\mathrm{k}))^{\wedge} 2\right]$;

\% Calculation of stiffness matrix for global coordinate system (x-y) as

$\%$ well as A, B and D matrices

disp('Stiffness matrix for each lamina with respect to the global coordinate system (x-y):')

Qbar=inv(T1)*Q*T2

epsilonL=T2*epsilonG

sigmaL $=Q^{*}$ epsilonL

\% Local stress (in GPa)

hQbar=Qbar* $(\mathrm{h}(\mathrm{k}+1)-\mathrm{h}(\mathrm{k}))$;

$\mathrm{A}=\mathrm{A}+\mathrm{hQbar}$;

h2Qbar=Qbar* $\left(\mathrm{h}(\mathrm{k}+1)^{\wedge} 2-\mathrm{h}(\mathrm{k})^{\wedge} 2\right)$;

$\mathrm{B}=\mathrm{B}+\mathrm{h} 2 \mathrm{Qbar}$;

h3Qbar=Qbar* $\left(h(k+1)^{\wedge} 3-h(k)^{\wedge} 3\right)$;

$\mathrm{D}=\mathrm{D}+\mathrm{h} 3 \mathrm{Qbar}$;

end

disp('Extensional stiffness matrix (A in GPa.mm), Coupling stiffness matrix (B in GPa.mm^2) and Bending stiffness matrix (D in GPa.mm^3), respectively:')

A

$\mathrm{B}=(1 / 2) * \mathrm{~B}$ 
$\mathrm{D}=(1 / 3) * \mathrm{D}$

$\mathrm{Ex}=(\mathrm{A}(1,1) * \mathrm{~A}(2,2)-\mathrm{A}(1,2) \wedge 2) /($ thickness $* \mathrm{~A}(2,2)) \%$ laminate stiffness

$\mathrm{Ey}=(\mathrm{A}(1,1) * \mathrm{~A}(2,2)-\mathrm{A}(1,2) \wedge 2) /($ thickness $* \mathrm{~A}(1,1)) \%$ laminate stiffness

$\mathrm{Gxy}=\mathrm{A}(3,3) /$ thickness \%laminate shear modulus

Nuxy $=A(1,2) / A(2,2) \%$ laminate poisson ratio

Nuyx $=A(1,2) / A(1,1) \%$ laminate poisson ratio

from above code find the below

Stiffness matrix for local coordinate system (L-T)

$$
Q=\left[\begin{array}{ccc}
135.6744 & 2.5699 & 0 \\
2.5699 & 8.2105 & 0 \\
0 & 0 & 2.9214
\end{array}\right]
$$

Compliance matrix for local coordinate system (L-T)

$$
S=\left[\begin{array}{ccc}
0.0074 & -0.0023 & 0 \\
-0.0023 & 0.1225 & 0 \\
0 & 0 & 0.3423
\end{array}\right]
$$




\begin{tabular}{|c|c|c|c|c|c|c|c|c|c|c|}
\hline \multirow[t]{2}{*}{ Specimen } & \multirow[t]{2}{*}{$\begin{array}{l}\text { Force } \\
(\mathbf{k N})\end{array}$} & \multicolumn{3}{|c|}{$\begin{array}{l}\text { Experimental Strain values in global } \\
\text { coordinate system from CCD camera images }\end{array}$} & \multicolumn{3}{|c|}{$\begin{array}{l}\text { Calculated Strain values } \\
\text { (Equation } 20 \text { ) in local coordinate } \\
\text { system for }-45 \text { degree angle ply }\end{array}$} & \multicolumn{3}{|c|}{$\begin{array}{l}\text { Calculated Strain values (Equation } \\
20 \text { ) in local coordinate system for } \\
\text { +45 degree angle ply }\end{array}$} \\
\hline & & Strain “ $x$ ” & Strain “y” & $\begin{array}{c}\text { Shear Strain } \\
\text { "xy" }\end{array}$ & $\begin{array}{c}\text { Strain } \\
\text { "L" }\end{array}$ & Strain "T" & $\begin{array}{c}\text { Shear Strain } \\
\text { "LT" }\end{array}$ & Strain "L" & Strain " $T$ " & $\begin{array}{c}\text { Shear Strain } \\
\text { "LT" }\end{array}$ \\
\hline \multirow[t]{5}{*}{ CM } & 7 & -0.00379169 & 0.00480729 & -0.00006891 & 0.0005 & 0.0005 & 0.0086 & 0.0005 & 0.0005 & -0.0086 \\
\hline & 9 & -0.00571597 & 0.00707902 & -0.0000793 & 0.0006 & 0.0007 & 0.0128 & 0.0007 & 0.0006 & -0.0128 \\
\hline & 10 & -0.00704433 & 0.0085911 & -0.000122994 & 0.0007 & 0.0008 & 0.0156 & 0.0008 & 0.0007 & -0.0156 \\
\hline & 11 & -0.008582 & 0.0103016 & -0.000130672 & 0.0008 & 0.0009 & 0.0189 & 0.0009 & 0.0008 & -0.0189 \\
\hline & 14 & -0.0210754 & 0.0233115 & -0.000316741 & 0.0010 & 0.0013 & 0.0444 & 0.0013 & 0.0010 & -0.0444 \\
\hline \multirow[t]{5}{*}{ AWJM } & 7 & -0.00370494 & 0.00490793 & -0.0000801867 & 0.0006 & 0.0006 & 0.0086 & 0.0006 & 0.0006 & -0.0086 \\
\hline & 9 & -0.00575873 & 0.00718998 & -0.000111655 & 0.0007 & 0.0008 & 0.0129 & 0.0008 & 0.0007 & -0.0129 \\
\hline & 10 & -0.00703579 & 0.00872131 & -0.000157349 & 0.0008 & 0.0009 & 0.0158 & 0.0009 & 0.0008 & -0.0158 \\
\hline & 11 & -0.00869734 & 0.010428 & -0.00017107 & 0.0008 & 0.0010 & 0.0191 & 0.0010 & 0.0008 & -0.0191 \\
\hline & 14 & -0.0226644 & 0.0249762 & -0.000516244 & 0.0009 & 0.0014 & 0.0476 & 0.0014 & 0.0009 & -0.0476 \\
\hline
\end{tabular}

Appendix A-2 Experimental and Calculated Strain values (Equation 20) in global and local coordinate system for +45 and -45 degree angle plies 


\begin{tabular}{|c|c|c|c|c|c|c|c|}
\hline \multirow[t]{2}{*}{ Specimen } & \multirow[t]{2}{*}{$\begin{array}{l}\text { Force } \\
(\mathbf{k N})\end{array}$} & \multicolumn{3}{|c|}{$\begin{array}{c}\text { Calculated Stress values (Equation } \\
24 \text { ) in local coordinate system for - } \\
45 \text { degree angle ply (GPa) }\end{array}$} & \multicolumn{3}{|c|}{$\begin{array}{l}\text { Calculated Stress values in local } \\
\text { (Equation 24) coordinate system } \\
\text { for }+45 \text { degree angle ply (GPa) } \\
\end{array}$} \\
\hline & & Stress "L" & Stress “T” & $\begin{array}{c}\text { Shear Stress } \\
\text { "LT" }\end{array}$ & Stress "L" & Stress "T" & $\begin{array}{c}\text { Shear } \\
\text { Stress "LT" }\end{array}$ \\
\hline \multirow[t]{5}{*}{ CM } & 7 & 0.0656 & 0.0057 & 0.0251 & 0.0748 & 0.0053 & -0.0251 \\
\hline & 9 & 0.0889 & 0.0076 & 0.0374 & 0.0995 & 0.0071 & -0.0374 \\
\hline & 10 & 0.0987 & 0.0087 & 0.0457 & 0.1151 & 0.0080 & -0.0457 \\
\hline & 11 & 0.1132 & 0.0096 & 0.0552 & 0.1276 & 0.0089 & -0.0552 \\
\hline & 14 & 0.1395 & 0.0129 & 0.1297 & 0.1746 & 0.0112 & -0.1297 \\
\hline \multirow[t]{5}{*}{ AWJM } & 7 & 0.0778 & 0.0067 & 0.0252 & 0.0885 & 0.0063 & -0.0252 \\
\hline & 9 & 0.0915 & 0.0080 & 0.0378 & 0.1064 & 0.0074 & -0.0378 \\
\hline & 10 & 0.1060 & 0.0095 & 0.0460 & 0.1270 & 0.0086 & -0.0460 \\
\hline & 11 & 0.1082 & 0.0098 & 0.0559 & 0.1310 & 0.0088 & -0.0559 \\
\hline & 14 & 0.1254 & 0.0139 & 0.1392 & 0.1624 & 0.0110 & -0.1392 \\
\hline
\end{tabular}

Appendix A-3 Calculated Stress values (from Equation 24) in local coordinate system for +45 and -45 degree angle plies 

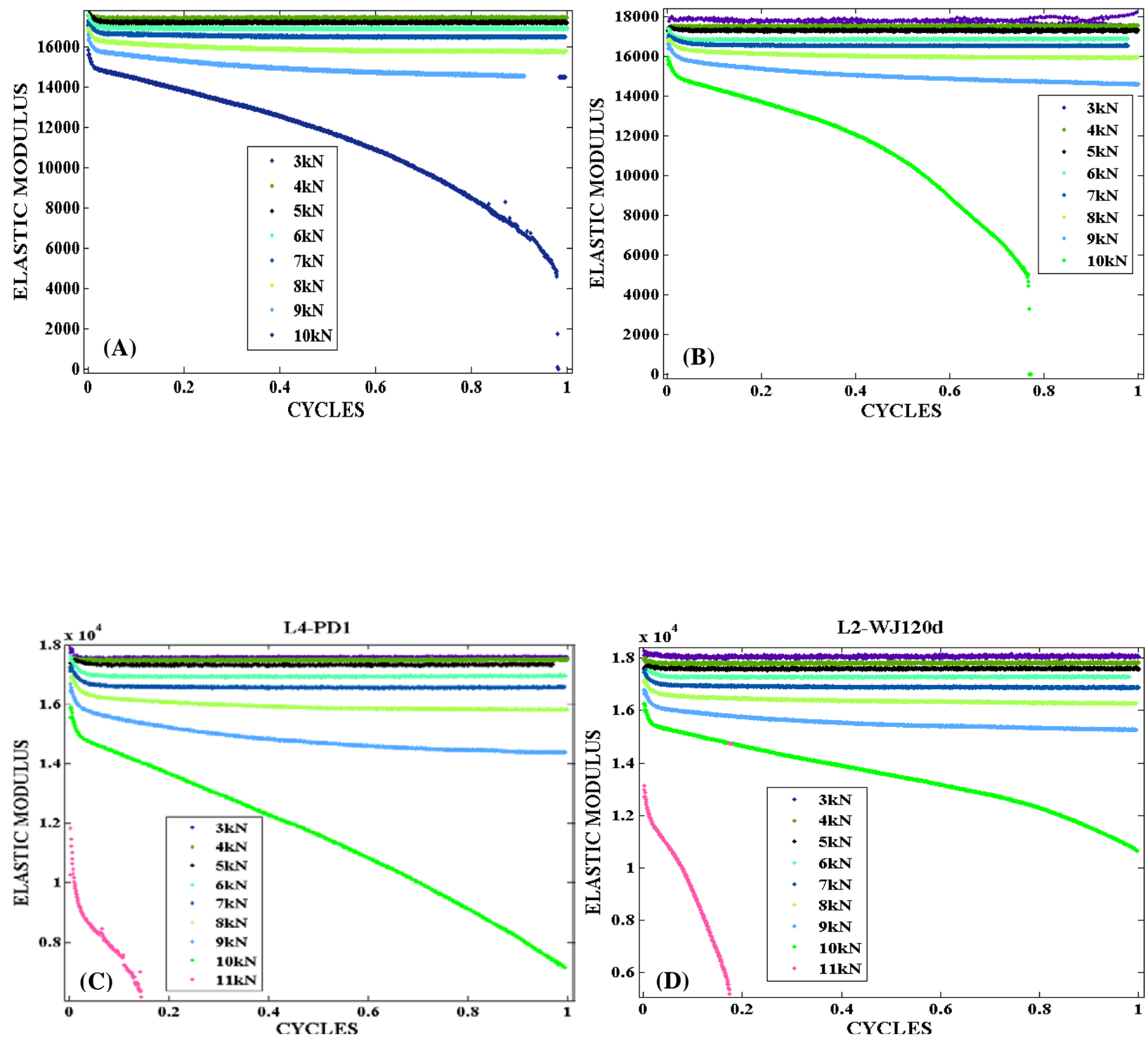

Appendix A-4 The elastic modulus vs normalized cycles for fatigue loading for (a) L1-CD1, (b) L2-PD1, (c) L4-PD1 and (d) L2-WJ120d specimens. 

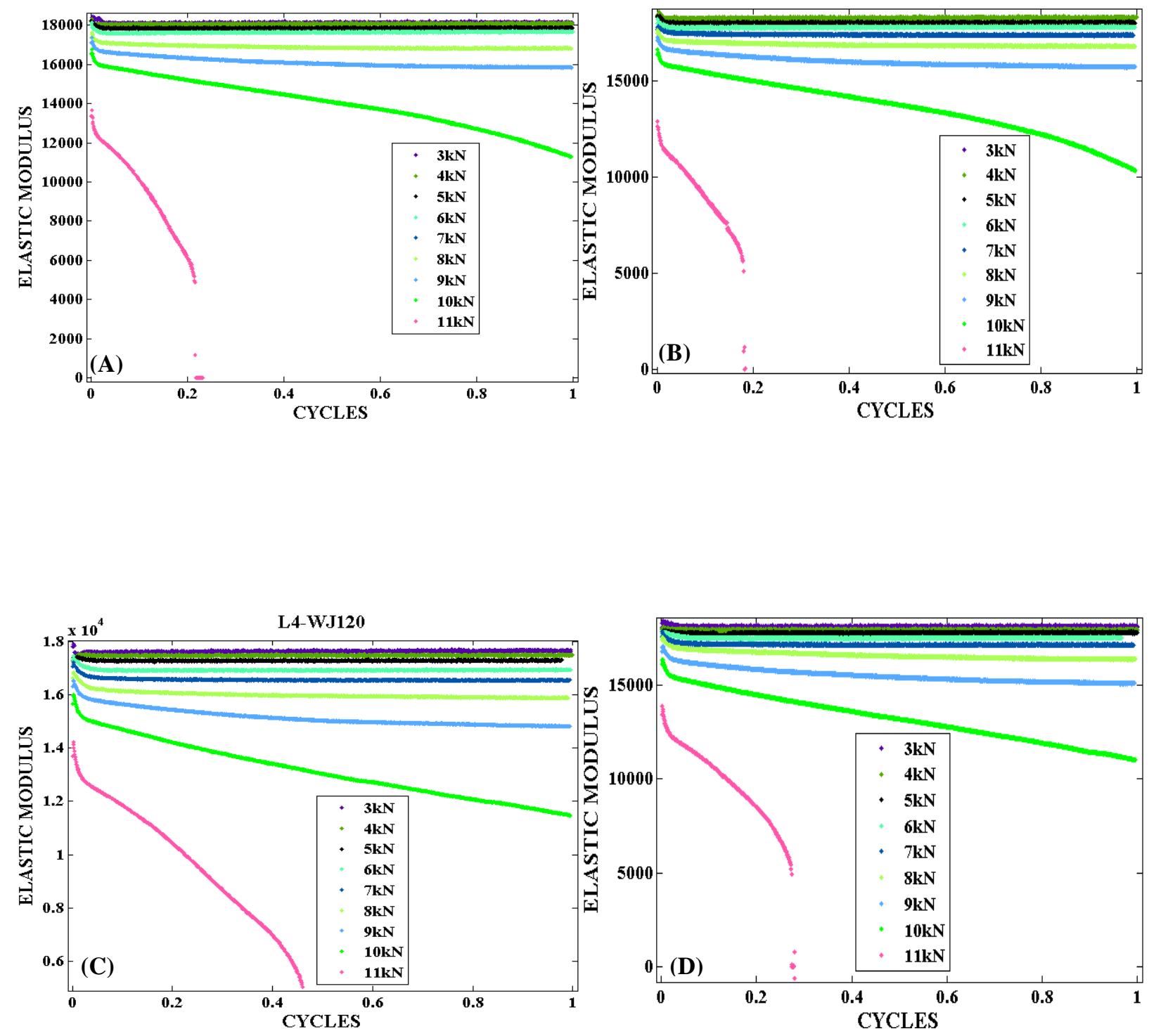

Appendix A-5 The elastic modulus vs normalized cycles for fatigue loading for (a) L2-WJ120, (b) L2-WJ220, (c) L4-WJ120 and (d) L4-WJ220 specimens. 

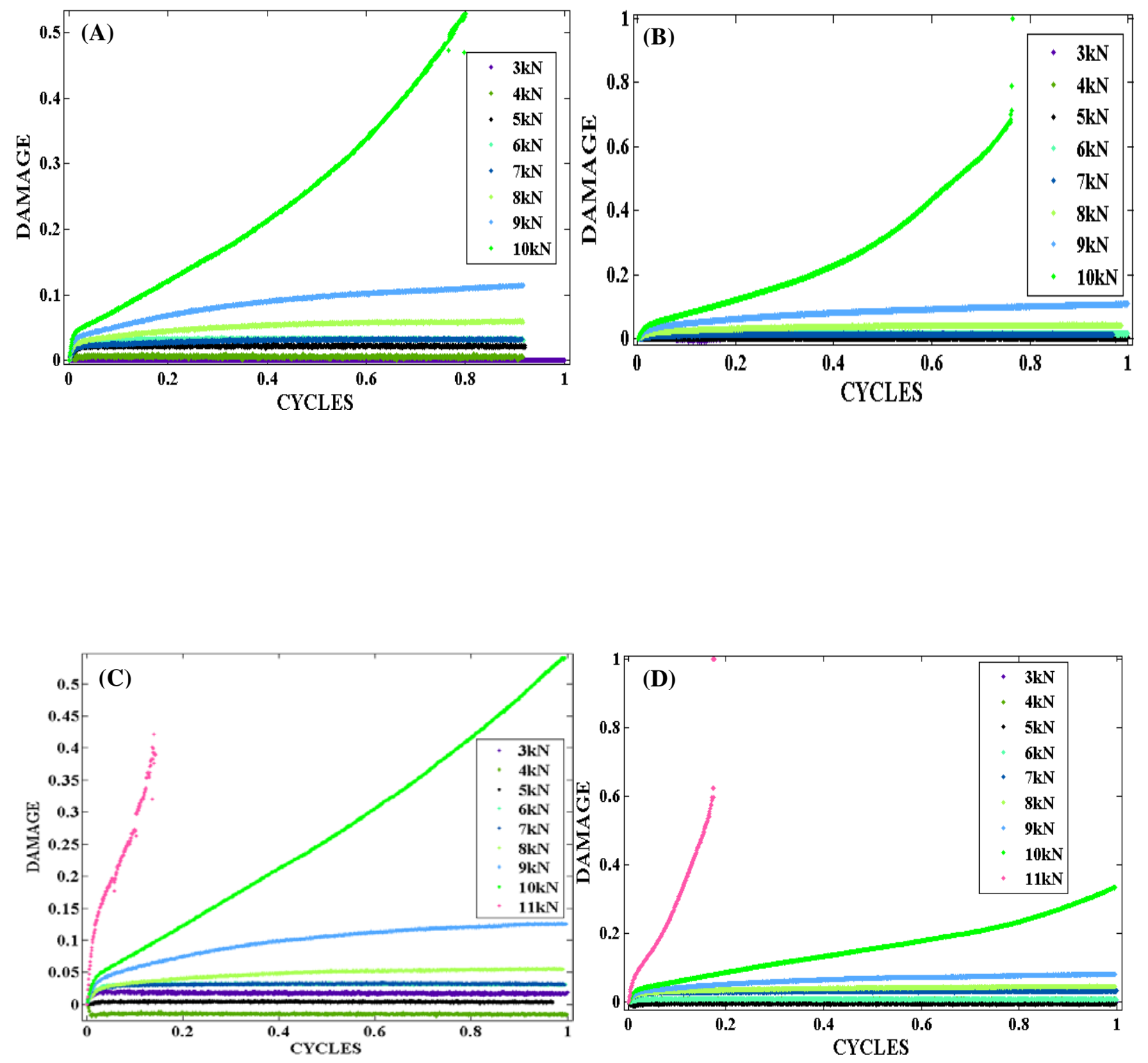

Appendix A-6 Comparison of the evolution of the damage for specimens (a) L1-CM1, (b) L2PD1, (c) L4-PD1 and (d) L2-WJ120d. 

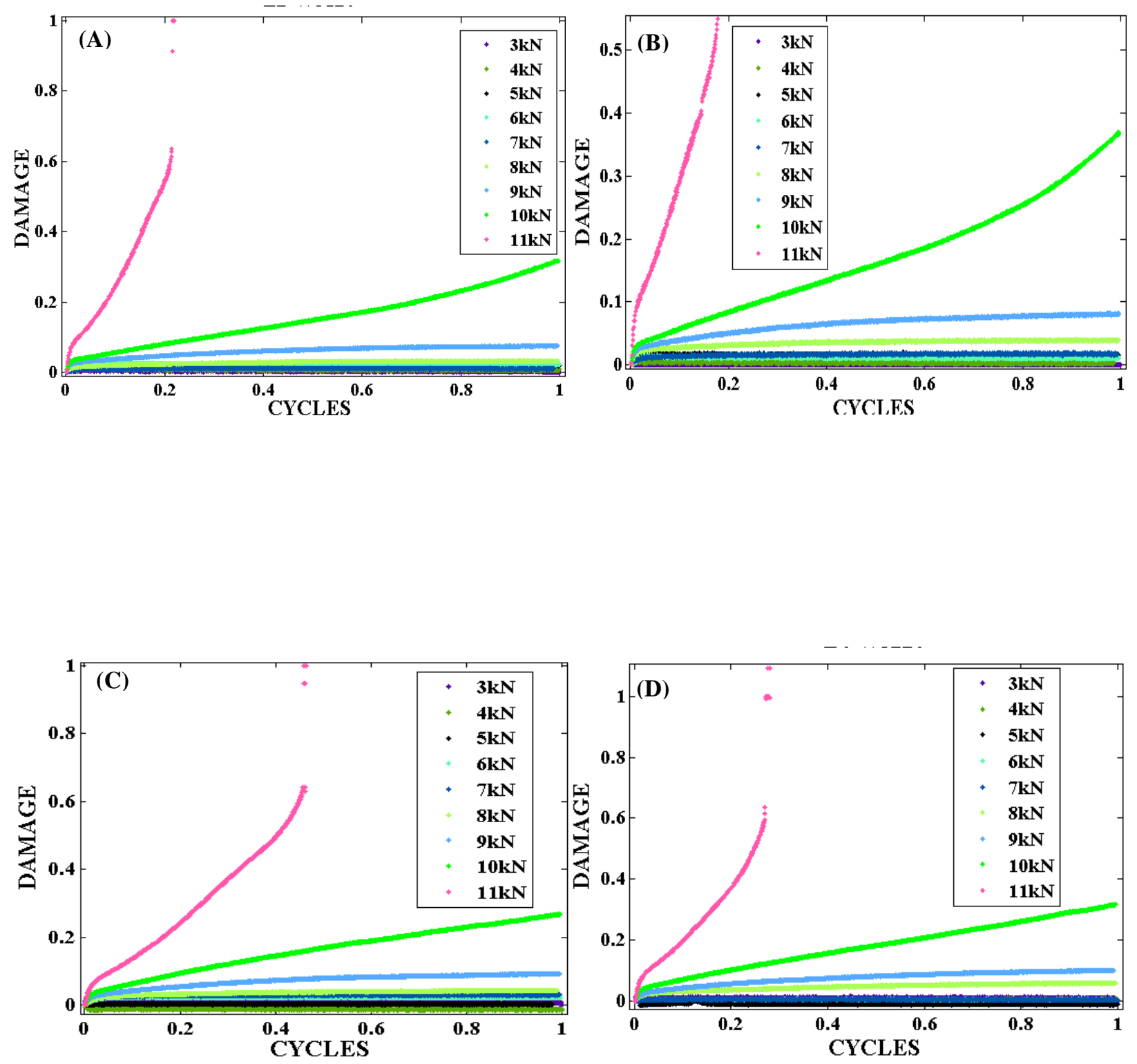

Appendix A-7 Comparison of the evolution of the damage for specimens (a) L2-WJ120, (b) L2WJ220, (c) L4-WJ120 and (d) L4-WJ220. 

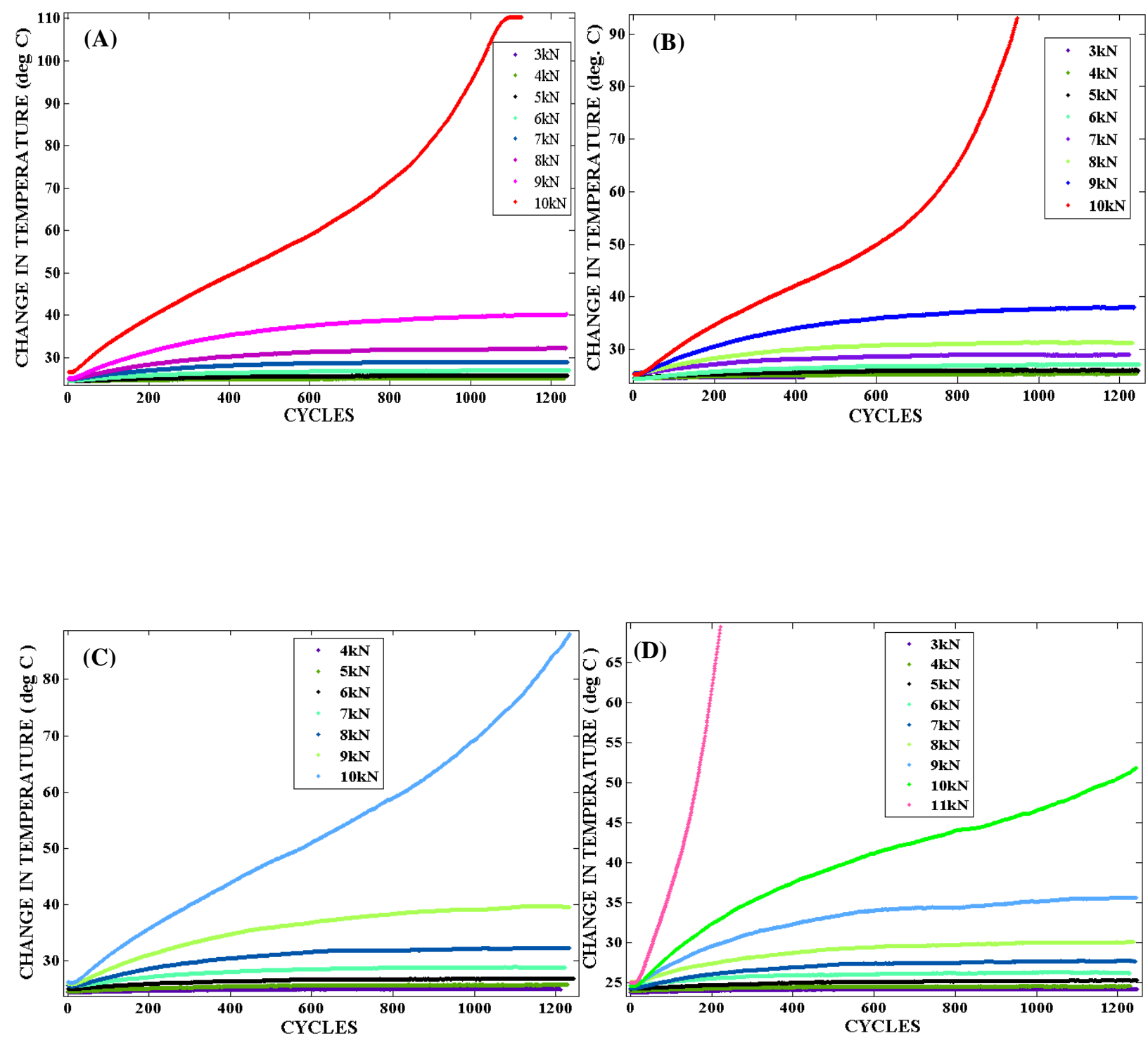

Appendix A-8 Various stages of the temperature for fatigue loading cases of 18, 24, 29, 35, 41, 47, 53, and 59 \% load to rupture for (a) L1-CM, (b) L2-PD1, (c) L4-PD1, (d) L2-WJ120d specimens. 

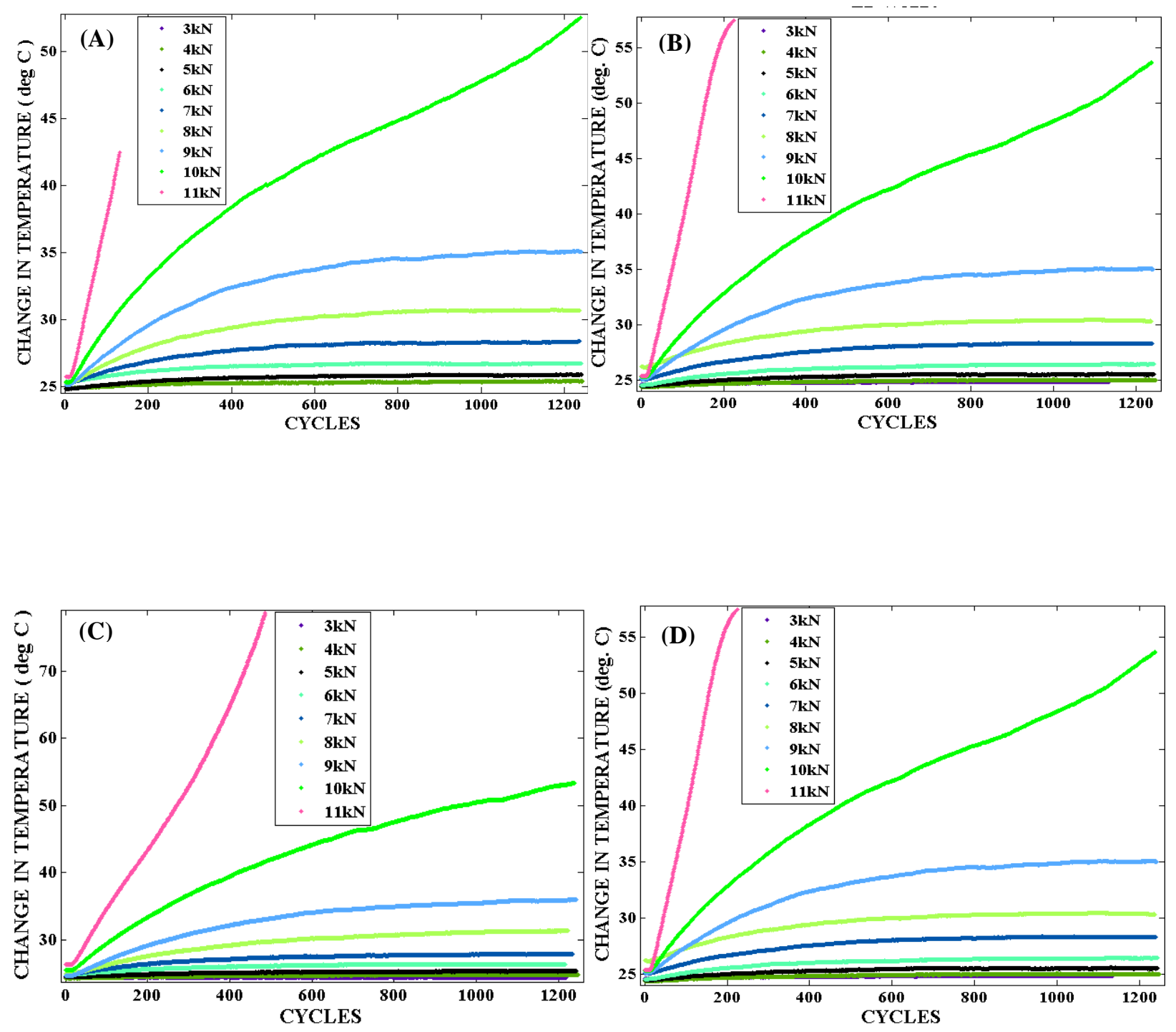

Appendix A-9 Various stages of stabilization of the temperature for fatigue loading cases of 18, 24, 29, 35, 41, 47, 53, and 59 \% load to rupture for (a) L2-WJ120, (b) L2-WJ220, (c) L4-WJ120, (d) L4-WJ220 specimens. 

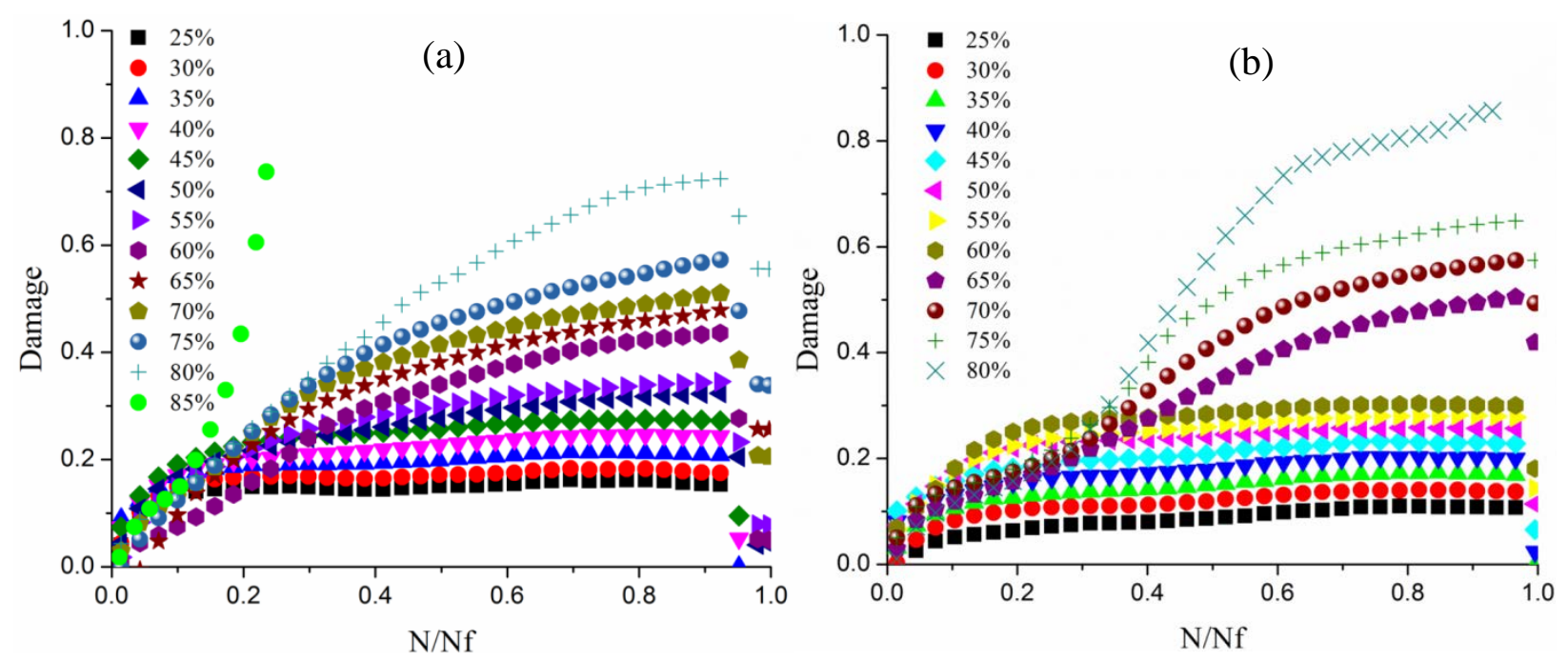

Appendix A-10 The evolution of the damage for various level of loading for Carbon/Epoxy (a) quasi isotropic and (b) angle ply lay-up assembly specimen tested at $10 \mathrm{~Hz}$ frequency for 10000 cycles, drilled with AWJM processes.
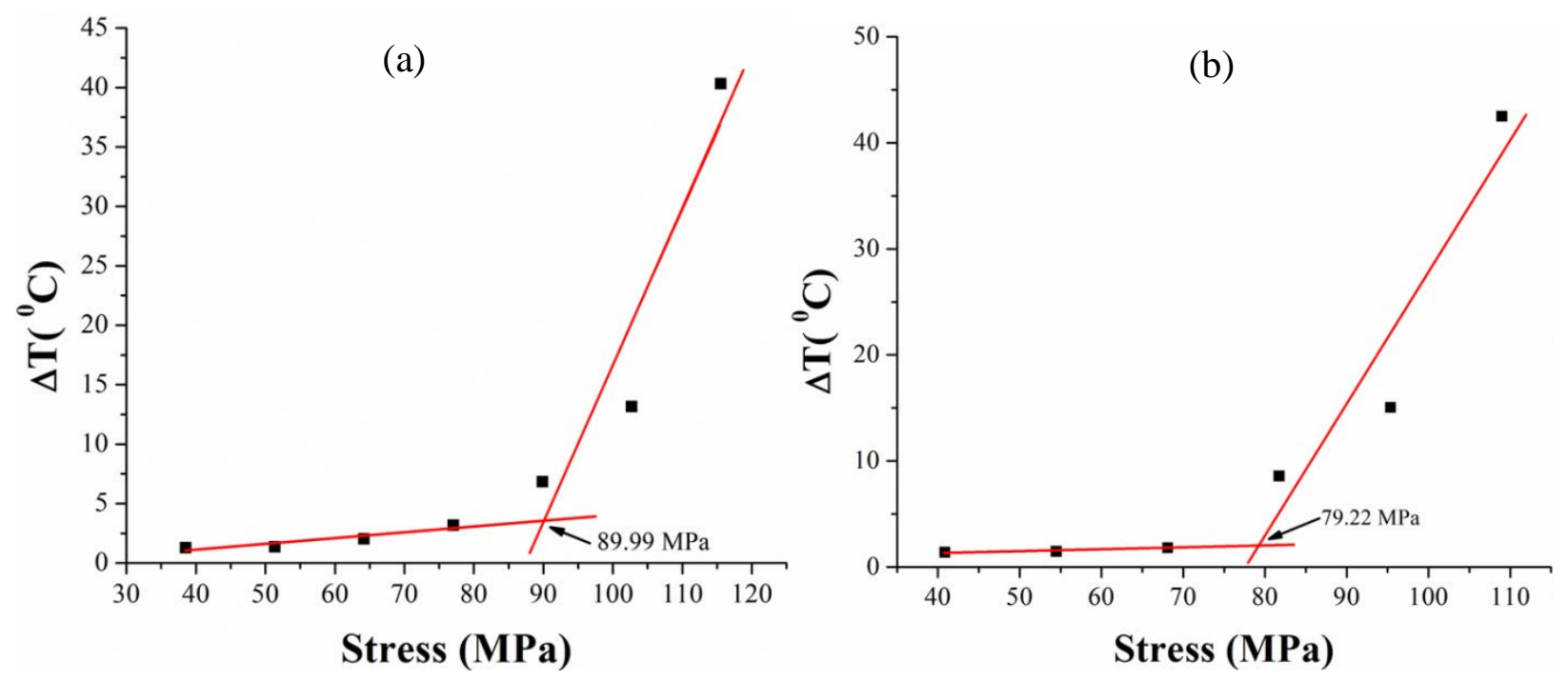

Appendix A-11 The endurance limit of $\left[ \pm 45^{\circ}\right]_{2 S}$ CF/Epoxy specimens machined with (a) AWJM and (b) CM technique, tested at $10 \mathrm{~Hz}$ frequency at 10000 cycles. 

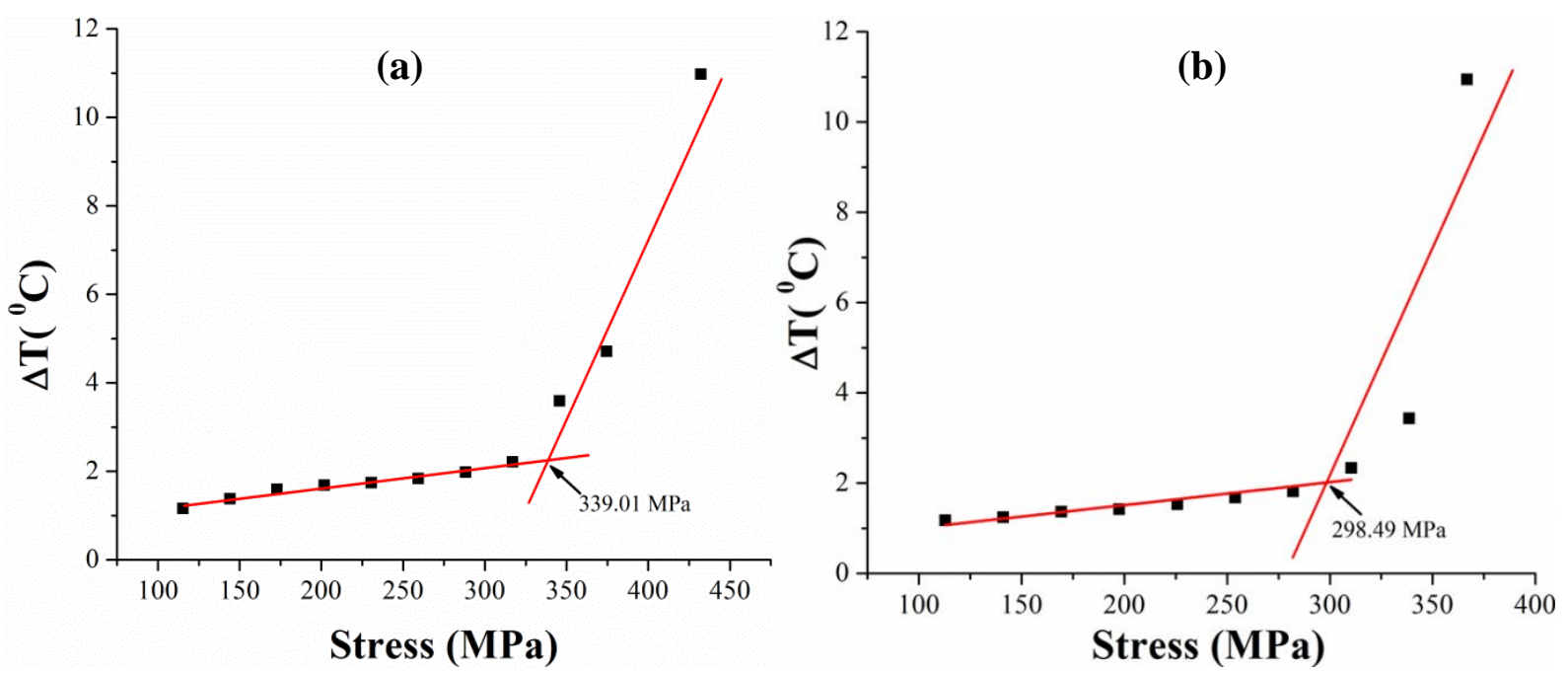

Appendix A-12 The endurance limit of [90/+45/0/-45] $]_{S}$ CF/Epoxy specimens machined with (a) AWJM and (b) CM technique, tested at $10 \mathrm{~Hz}$ frequency and at 10000 cycles. 


\section{Appendix A-13, List of Publications}

\section{List of Journal Paper, Book Section:}

M. Saleem, L. Toubal, R. Zitoune, H. Bougherara, Investigating the effect of machining processes on the mechanical behaviour of composite plates with circular holes, Composites A: Applied Science and Manufacturing, Vol. 55 December, 2013. p. 169-177, DOI: 10.1016/j.compositesa.2013.09.002. ISSN: 1359-835X (5-Year Impact Factor: 3.453)

M. Saleem, H. Bougherara, L. Toubal, F. Cénac, R. Zitoune, Analysis of Stresses in CFRP Composite Plates Drilled with Conventional and Abrasive Water-jet Machining, In book Materials Science Forum - Vol 763, p.127-143. Ed. Trans Tech Publications, Switzerland. 2013 ISBN:doi:10.4028/www.scientific.net/MSF.763.127

\section{List of Papers under preparation:}

Influence of conventional and abrasive water-jet machining processes on the damage behaviour of the CFRP composite single-lap, single-bolted joint assembly.

Effect of Conventional and Abrasive Water-jet machining technique on the damage behaviour of different stacking sequence of Carbon/Epoxy Composite Material

\section{Conference Proceedings/Presentation:}

M. Saleem, L. Toubal, R. Zitoune, H. Bougherara, Thermographic evaluation of CFRP specimens drilled with conventional and abrasive water-jet techniques, in proceedings of the $19^{\text {th }}$ International Conference on Composite materials (ICCM), Montreal, Canada, July 28 August 2, 2013.

S. Muhammad, L. Toubal, R. Zitoune, H. Bougherara and F. Cénac, Influence of experimental drilling process on the mechanical behaviour of CFRP composite plate, Presented in; $15^{\mathrm{TH}}$ European Conference on Composite Materials (ECCM 15), Venice, Italy. June 24-28 2012. 


\section{Bibliography}

[1] Zitoune R, Krishnaraj V, Collombet F. Study of drilling of composite material and aluminium stack. Composite Structures 2010; 92: 1246-1255

[2] Zitoune R, Crouzeix L, Collombet F, Tamine T, Grunevald Y-. Behaviour of composite plates with drilled and moulded hole under tensile load. Composite Structures 2011

[3] Mohamed H. Abrasive waterjet cutting has grown along with the increased use of composites in airframes, and advanced techniques help make it a preferred technology. Cutting Tool Engineering 2009; 61: 43-48

[4] Bonello K.J. Damage accumulation in Graphite/Epoxy laminates due to cyclic gradient stress fields, Master of science thesis, Massachusetts Institute of Technology. Dept. of Aeronautics and Astronautics 1988

[5] Schon J, Nyman T. Spectrum fatigue of composite bolted joints. International Journal of Fatigue 2002; 24: 273-279

[6] Sheikh-Ahmad JY. ed. Machining of Polymer Composites, ISBN 978-0-387-35539-9. New York, USA. ISBN: 978-0-387-35539-9: Springer. 2009

[7] Minnetyan L. Damage Progression in Bolted Composites. Journal of thermoplastic composite materials May 1998; 11: 231-248

[8] Venkayya VB, Ramkumar RL, Tischler VA, Snyder BD, Burns JG. "Recent studies on bolted joints in composite structures", in "Behaviour and analysis of mechanically fastened joints in composite structures” 1987; 427: 43-56

[9] Bi ZM, Hinds B, Jin Y, Gibson R, McToal P. ed. Studies on composites drilling-The state of the art: Nova Science Publishers. 2009 
[10] Zitoune R, Collombet F. Numerical prediction of the thrust force responsible of delamination during the drilling of the long-fibre composite structures. Composites Part A: Applied Science and Manufacturing 2007; 38: 858-866

[11] Persson E, Eriksson I, Zackrisson L. Effects of hole machining defects on strength and fatigue life of composite laminates. Composites Part A: Applied Science and Manufacturing 1997; 28: 141-151

[12] König W, Wulf C, Graß P, Willerscheid H. Machining of Fibre Reinforced Plastics. CIRP Annals - Manufacturing Technology 1985; 34: 537-548

[13] Davim JP, Reis P. Drilling carbon fiber reinforced plastics manufactured by autoclaveexperimental and statistical study. Materials and Design 2003; 24: 315-324

[14] Zitoune R, Collombet F, Lachaud F, Piquet R, Pasquet P. Experiment-calculation comparison of the cutting conditions representative of the long fiber composite drilling phase. Composites Science and Technology 2005; 65: 455-466

[15] Abrate S, Walton DA. Machining of composite materials. Part I: Traditional methods. Composites Manufacturing 1992; 3: 75-83

[16] Sanjay KM. Composites Manufacturing Materials Product and Process Engineering: ISBN 0-8493-0585-3. 2002

[17] Ramulu M, Arola D. The influence of abrasive waterjet cutting conditions on the surface quality of graphite/epoxy laminates. International Journal of Machine Tools and Manufacture 1994; 34: 295-313

[18] Arola D, Mccain ML. Surface texture and the stress concentration factor for FRP components with holes. Journal of Composite Materials 2003; 37: 1439-1460

[19] Bhatnagar N, Nayak D, Singh I, Chouhan H, Mahajan P. Determination of machininginduced damage characteristics of fiber reinforced plastic composite laminates. Materials and Manufacturing Processes 2004; 19: 1009-1023 
[20] Torres M, Gonzalez JL, Hernandez H. Residual strength and fracture path for drilled epoxyglass composites. Advanced Materials Research 2009; 65: 89-96

[21] Axinte DA, Srinivasu DS, Kong MC, Butler-Smith PW. Abrasive waterjet cutting of polycrystalline diamond: A preliminary investigation. International Journal of Machine Tools and Manufacture 2009; 49: 797-803

[22] Abdel-Rahman AA. A Closed-form Expression for an abrasive waterjet cutting model for ceramic materials. International Journal of Mathematical Models and Methods in Applied Sciences 2011; 5: 722-729

[23] Fordham JD, Pilkington R, Tang CC. The effect of different profiling techniques on the fatigue performance of metallic membranes of AISI 301 and Inconel 718. International Journal of Fatigue 1997; 19: 487-501

[24] Brooghani SY, Hassanzadeh H, Kahhal P. Modeling of single particle impact in abrasive water jet machining 2007; 36: 243-248

[25] Horn GP, Mackin TJ, Kurath P. Composite machining damage quantification using thermoelastic stress analysis. Polymer Composites 2002; 23: 193-200

[26] Toubal L, Karama M, Lorrain B. Damage evolution and infrared thermography in woven composite laminates under fatigue loading. International Journal of Fatigue 2006; 28: 1867-1872

[27] Ocnarescu M, Spanu P, Vlase A, Opran C. Machining of composite materials using traditional methods in " Nonconventional Technologies Review " ed. Aurel JM. 2008; 3: 43-46

[28] Wern CW, Ramulu M, Colligan K. A study of the surface texture of composite drilled holes . Journal of Materials Processing Technology 1993; 37: 373-389

[29] Tsao CC, Hocheng H. Taguchi analysis of delamination associated with various drill bits in drilling of composite material. International Journal of Machine Tools and Manufacture 2004; 44: $1085-1090$ 
[30] Kim D, Ramulu M, Doan X. Influence of consolidation process on the drilling performance and machinability of PIXA-M and PEEK thermoplastic composites. Journal of Thermoplastic Composite Materials 2005; 18: 195-217

[31] Davim JP, Reis P, António CC. Experimental study of drilling glass fiber reinforced plastics (GFRP) manufactured by hand lay-up. Composites Science and Technology 2004; 64: 289-297

[32] Gaitonde VN, Karnik SR, Campos Rubio J, Correia AE, Abro AM, Davim JP. Analysis of parametric influence on delamination in high-speed drilling of carbon fiber reinforced plastic composites. J Mater Process Technology 2008; 203: 431-8

[33] Campos Rubio J, Abrao AM, Faria PE, Correia AE, Davim JP. Effects of high speed in the drilling of glass fibre reinforced plastic: Evaluation of the delamination factor. International Journal of Machine Tools and Manufacture 2008; 48: 715-720

[34] Krishnaraj V, Vijayarangan S, Ramesh Kumar A. Effect of drilling parameters on mechanical strength in drilling glass fibre reinforced plastic. International Journal of Computer Applications in Technology 2007; 28: 87-93

[35] El-Sonbaty I, Khashaba UA, Machaly T. Factors affecting the machinability of GFR/epoxy composites. Composite Structures 2004; 63: 329-38

[36] Jain S, Yang DCH. Delamination free drilling of composite laminates. Processing, Fabrication, and Manufacturing of Composite Materials 1992; 35

[37] Lin S-Chien. SJ. Drilling Unidirectional Glass Fiber-Reinforced Composite Materials at High Speed. Journal of Composite materials 1999; 33: 827-851

[38] Fernandes M, Cook C. Drilling of carbon composites using a one shot drill bit. Part I: Five stage representation of drilling and factors affecting maximum force and torque. International Journal of Machine Tools and Manufacture 2006; 46: 70-75 
[39] Zhang L-, Wang L-, Liu X-. A mechanical model for predicting critical thrust forces in drilling composite laminates. Proceedings of the Institution of Mechanical Engineers, Part B: Journal of Engineering Manufacture 2001; 215: 135-144

[40] Isbilir O GE. Finite element analysis of drilling of carbon fibre reinforced composites. Appl Compos Mater 2012; 19: 637-56

[41] Ho-Cheng H, Dharan CKH. Delamination during drilling in composite laminates. Journal of engineering for industry 1990; 112: 236-239

[42] Lin SC, Chen IK. Drilling carbon fiber-reinforced composite material at high speed. Wear 1996; 194: 156-162

[43] Dharan CKH, Won MS. Machining parameters for an intelligent machining system for composite laminates. International Journal of Machine Tools and Manufacture 2000; 40: 415426

[44] Zhang H, Chen W, Chen D, Zhang L. Assessment of the exit defects in carbon fibrereinforced plastic plates caused by drilling. Key Engineering Materials 2001; 196: 43-52

[45] Singh I, Bhatnagar N, Viswanath P. Drilling of uni-directional glass fiber reinforced plastics: Experimental and finite element study. Materials and Design 2008; 29: 546-553

[46] Piquet R, Ferret B, Lachaud F, Swider P. Experimental analysis of drilling damage in thin carbon/epoxy plate using special drills. Composites Part A: Applied Science and Manufacturing 2000; 31: 1107-1115

[47] Langella A, Nele L, Maio A. A torque and thrust prediction model for drilling of composite materials. Composites Part A: Applied Science and Manufacturing 2005; 36: 83-93

[48] Di Ilio A, Paoletti A, Veniali F. Tool wear in drilling thermoset and thermoplastic matrix composites. American Society of Mechanical Engineers, Petroleum Division (Publication) PD 1996; 75: 41-46 
[49] Davim JP, Reis P. Study of delamination in drilling carbon fiber reinforced plastics (CFRP) using design experiments. Composite Structures 2003; 59: 481-487

[50] Davim JP. Study of drilling metal-matrix composites based on the Taguchi techniques. Journal of Materials Processing Technology 2003; 132: 250-254

[51] Chen W-. Some experimental investigations in the drilling of carbon fiber-reinforced plastic (CFRP) composite laminates. International Journal of Machine Tools and Manufacture 1997; 37: 1097-1108

[52] Kilickap E. Optimization of cutting parameters on delamination based on Taguchi method during drilling of GFRP composite. Exp Syst Appl 2010; 37: 6116-22

[53] A. Di Ilio, A. Paoletti. Damage reduction methods in drilling polymeric matrix composites 2009: 45-55

[54] Rivero A, Ostra T, Rodriguez G, Lechner M. Delamination phenomenon during drilling of carbon fiber composites in"Drilling of Composite Materials" ed.Davim JP. 2009: 96-110

[55] Manna A, Mohindra V, Patra S, Sandeep M. Drilling of e-glass fibre reinforced polymer composite. International Journal of Machining and Machinability of Materials 2008; 3: 343-355

[56] Wang X, Wang LJ, Tao JP. Investigation on thrust in vibration drilling of fiber-reinforced plastics. Journal of Materials Processing Technology 2004; 148: 239-244

[57] Tercan M, Asi O, Aktaş A. Determination of the critical crack length of notched weftknitted glass fiber variable width composite plates. Composite Structures 2007; 77: 111-119

[58] Whitney JM, Nuismer RJ. Stress fracture criteria for laminated composites containing stress concentrations. Journal of Composite Materials 1974; 8: 253-265

[59] Toubal L, Karama M, Lorrain B. Stress concentration in a circular hole in composite plate. Composite Structures 2005; 68: 31-36 
[60] Mohd Ariffin MKA, Mohd Ali MI, Sapuan SM, Ismail N. An optimise drilling process for an aircraft composite structure using design of experiments. Scientific Research and Essays 2009; 4: 1109-1116

[61] Hossain ME, Ketata C, Khan MI. Islam MR. A sustainable drilling technique. Journal of Nature Science and Sustainable Technology 2010; 4: 73-93

[62] Zitoune R CF. Drilling of composite materials In: Influence of machining quality on composite part manufacturing . 2009

[63] Arola DD, Ramulu M. Net-Shape Machining and the Process-Dependent Failure of FiberReinforced Plastics under Static Loads. Journal of Composites Technology and Research 1998; 20: $210-220$

[64] Ghidossi P, Mansori ME, Pierron F. Influence of specimen preparation by machining on the failure of polymer matrix off-axis tensile coupons. Composites Science and Technology 2006; 66: $1857-1872$

[65] D.M.S.I. Diversified metal services incorporation, http://www.diversifiedmetalservices.com/waterjet.html; 2012

[66] Youssef HA. Machining technology : machine tools and operations. 6000 Broken Sound Parkway NW, Suite 300 Boca Raton, FL: CRC Press. 2008

[67] Geng H. Manufacturing Engineering Handbook (2): The McGraw-Hill Companies, DOI: 10.1036/0071398252. 2004

[68] Durão LMP, Gonçalves DJS, Tavares JMRS, Albuquerque, VHC, Vieira AA, Marques AT. Drilling tool geometry evaluation for reinforced composite laminates. Composite Structures 2010; 92

[69] Schwartz M. Composite Material Handbook: McGraw-Hill, New York USA. McGraw-Hill, New York USA,1992 
[70] Liu H-. Hole drilling with abrasive fluidjets. International Journal of Advanced Manufacturing Technology 2007; 32: 942-957

[71] Jain NK, Jain VK. Modeling of material removal in mechanical type advanced machining processes: A state-of-art review. International Journal of Machine Tools and Manufacture 2001; 41: 1573-1635

[72] Hashish M. Milling with abrasive-waterjets: a preliminary investigation. In. Proceedings of the fourth U.S. Water Jet Conference, Berkeley, California, pp. 179-188 1987

[73] Griffiths B. Manufacturing surface technology: Surface integrity and functional performance: Penton Press, an imprint of Kogan Page Ltd. 2001

[74] Mark J.J DJP, ed. Machining with Abrasives, ISBN 978-1-4419-7301-6: Springer New York. 2011

[75] Shipway PH, Fowler G, Pashby IR. Characteristics of the surface of a titanium alloy following milling with abrasive waterjets. Wear 2005; 258: 123-32

[76] Fowler G. Abrasive water-jet - controlled depth milling of titanium alloys, PhD thesis, University of Nottingham, School of Mechanical, Materials, Manufacturing, Engineering and Management July 2003

[77] Arola D, Ramulu M, Arola DD. Material removal in abrasive waterjet machining of metals surface integrity and texture. Wear 1997; 210: 50-58

[78] Marinescu ID, Rowe WB, Dimitrov B, Inasaki I. ed. Tribology of abrasive machining process. United States of America: William Andrew, Inc. 2004

[79] Sheikh-Ahmad JY. Machining of polymer composites. New York: Springer. 2009

[80] Bayoumi MR, Abdellatif AK. Effect of surface finish on fatigue strength. Engineering Fracture Mechanics 1995; 51: 861-70 
[81] Davim JP, Reis P, António CC. Drilling fiber reinforced plastics (FRPs) manufactured by hand lay-up: Influence of matrix (Viapal VHP 9731 and ATLAC 382-05). Journal of Materials Processing Technology 2004; 155-156: 1828-1833

[82] Davim JP, Reis P. Damage and dimensional precision on milling carbon fiber-reinforced plastics using design experiments. Journal of Materials Processing Technology 2005; 160: 160167

[83] Davim JP, Mata F. Influence of cutting parameters on surface roughness in turning glassfibre-reinforced plastics using statistical analysis. Industrial Lubrication and Tribology 2004; 56: $270-274$

[84] Latha B, Senthilkumar VS. Modeling and analysis of surface roughness parameters in drilling GFRP composites using fuzzy logic. Materials and Manufacturing Processes 2010; 25: 817-827

[85] Palanikumar K, Mata F, Davim JP. Analysis of surface roughness parameters in turning of FRP tubes by PCD tool. Journal of Materials Processing Technology 2008; 204: 469-474

[86] Tsao CC, Hocheng H. Evaluation of thrust force and surface roughness in drilling composite material using Taguchi analysis and neural network. Journal of Materials Processing Technology 2008; 203: 342-348

[87] Khashaba UA, El-Sonbaty IA, Selmy AI, Megahed AA. Machinability analysis in drilling woven GFR/epoxy composites: Part I - Effect of machining parameters. Composites Part A: Applied Science and Manufacturing 2010; 41: 391-400

[88] Anwar S, Axinte DA, Becker AA. Finite element modelling of a single-particle impact during abrasive waterjet milling 2011; 225: 821-832

[89] Lawlor VP, Stanley WF, McCarthy MA. Characterisation of damage development in single shear bolted composite joints. Journal of Plastics, Rubber and Composites 2002; 31: 126-133 
[90] Lawlor VP, McCarthy MA, Stanley WF. Experimental study on the effects of clearance on single-bolt, single-shear, composite bolted Joints. Journal of Plastics, Rubber and Composites; 31: $405-411$

[91] Bauer J. "Mechanism of single shear fastened joints", in "Behaviour and analysis of mechanicallyfastened joints in composite structures" 1987; 427: 234-239

[92] Starikov R, Schön J. Local fatigue behaviour of CFRP bolted joints. Composites Science and Technology February 2002; 62: 243-253

[93] Jiang Y, Zhang M, Lee C.-H. A study of early stage self-loosening of bolted joints. Journal of Mechanical Design Sept. 2003; 125: 518-526

[94] Cooper C, Turvey GJ. Effects of joint geometry and bolt torque on the structural performance of single bolt tension joints in pultruded GRP sheet material. Composite Structures 1995; 32: 217-226

[95] Persson EE, I. Fatigue of multiple-row bolted joints in carbon/epoxy laminates: ranking of factors affecting strength and fatigue life. International Journal of Fatigue April 1999; 21: 337353

[96] Kelly G, Hallström S. Bearing strength of carbon fibre/epoxy laminates: effects of bolt-hole clearance. Composites: Part B 2004; 35: 331-343

[97] Smith PA, Pascoe KJ. Fatigue of Bolted Joints in (0/90) CFRP Laminates. Composites Science and Technology 1987; 29: 45-69

[98] Scalea D, Lanza F, Cloud GL, Cappello F. A study on the effects of clearance and interference fits in a pin-loaded cross-ply FGRP laminate 1998; 32: 783-802

[99] Banbury A, Kelly DW. A study of fastener pull-through failure of composite laminates. Part 1: Experimental. Composite Structures 1999; 45: 241-254 
[100] Lim TS, Kim BC, Lee DG. Fatigue characteristics of the bolted joints for unidirectional composite laminates. Composite Structures Jan. 2006; 72: 58-68

[101] McCarthy MA, Lawlor VP, Stanley WF, McCarthy CT. Bolt-hole clearance effects and strength criteria in single-bolt, single-lap, composite bolted joints. Composites Science and Technology August 2002; 62: 1415-1431

[102] Saunders DS, Galea SC, Deirmendjian GK. Development of fatigue damage around fastener holes in thick graphite/epoxy composite laminates. Composites Jun 1993; 24: 309-321

[103] Starikov R, Schön J. Experimental study on fatigue resistance of composite joints with protruding head bolts. Composite Structures 2002; 55: 1-11

[104] Okutan B. The effects of geometric parameters on the failure strength for pin-loaded multidirectional fiber-glass reinforced epoxy laminate. Composites Part B: Engineering December 2002; 33: 567-578

[105] Aktas A. Failure analysis of serial pinned joints in composite materials. Indian Journal of Engineering and Materials Sciences April 2011; 18: 102-110

[106] H. Mao SM. Fatigue damage modelling of composite materials. Composite Structures 2002; 58: 405-410

[107] Abdel-Latif A.M. An overview of the applications of NDI/NDT in engineering design of structural integrity and damage tolerance in aircraft structures. Damage and Fracture Mechanics: Failure Analysis of Engineering Materials and Structures 2009: 93-100

[108] Yao WX, Himmel N. A new cumulative fatigue damage model for fibre-reinforced plastics. Composites Science and Technology 2000; 60: 59-64

[109] Ye L. On fatigue damage accumulation and material degradation in composite materials. Composites Science and Technology 1989; 36: 339-350 
[110] Varvani-Farahani A, Shirazi A. A fatigue damage model for (0/90) FRP composites based on stiffness degradation of $0^{\circ}$ and $90^{\circ}$ composite plies. Journal of Reinforced Plastics and Composites 2007; 26: 1319-1336

[111] Saleem M, Toubal L, Zitoune R, Bougherara H. Investigating the effect of machining processes on the mechanical behavior of composite plates with circular holes. Composites Part A: Applied Science and Manufacturing 2013; 55: 169-177

[112] Haddad M, Zitoune R, Bougherara H, Eyma F, Castanié B. Study of trimming damages of CFRP structures in function of the machining processes and their impact on the mechanical behavior . Composites: Part B 2014; 57: 136-143

[113] Hamdoun Z, Guillaumat L, Lataillade J-. Influence of the drilling quality on the fatigue compression behaviour of carbon epoxy laminates. International Journal of Fatigue 2006; 28: 1-8

[114] Toubal L. Analytical and experimental approaches of the damage by fatigue of a composite carbon/epoxy 2004: 2130

[115] Vijayan K, Zitoune R. Davim JP, ed. Drilling of polymer matrix composites: ISBN 978-184821-170-4, John Wiley \& Sons Inc: Springer. 2010

[116] ASTM Standard D5766/D5766M - 11 (2007) Standard Test Method for Open-Hole Tensile Strength of Polymer Matrix Composite Laminates

[117] ASTM Standard D5961/D5961M - 10 (2010) Standard Test Method for Bearing Response of Polymer Matrix Composite Laminates

[118] ASTM Standard D3039/D3039 m-08 (2007) Standard test method for tensile properties of polymer matrix composite materials.

[119] Pandita SD, Nishiyabu K, Verpoest I. Strain concentrations in woven fabric composites with holes. Composite Structures 2003; 59: 361-368 
[120] Fargione G, Geraci A, La Rosa G, Risitano A. Rapid determination of the fatigue curve by the thermographic method. International Journal of Fatigue 2002; 24: 11-19

[121] Krapez J-C, Pacou D, Bertin C. Application of lock-in thermography to rapid evaluation of fatigue limit in metals. Workshop on Advanced Infrared Technology and Applications Venice (Italy) September 28-30, 1999

[122] Luong MP. Infrared thermographic scanning of fatigue in metals. Nuclear Engineering and Design 1995; 158: 363-376

[123] Luong MP. Fatigue limit evaluation of metals using an infrared thermographic technique. Mechanics of Materials 1998; 28: 155-163

[124] La Rosa G, Risitano A. Thermographic methodology for rapid determination of the fatigue limit of materials and mechanical components. International Journal of Fatigue 2000; 22: 65-73

[125] Liaw PK, Wang H, Jiang L, Yang B, Huang JY, Kuo RC, Huang JG. Thermographic detection of fatigue damage of pressure vessel steels at 1,000 Hz and $20 \mathrm{~Hz}$. Scripta Materialia 2000; 42: 389-395

[126] Yang B, Liaw PK, Wang H, Jiang L, Huang JY, Kuo RC, Huang JG. Thermographic investigation of the fatigue behavior of reactor pressure vessel steels. Materials Science and Engineering A 2001; 314: 131-139

[127] Kurashiki K, Ni Q-, Iwamoto M. Fatigue Damage Evaluation of GFRP Using Infrared Thermography - Effects of Matrix Resin and Fiber Content on Evaluation of Fatigue Damage. Journal of the Society of Materials Science, Japan 2003; 52: 1253-1257

[128] Chrysochoos A. Infrared thermography, a potential tool for analysing the material behaviour. Mecanique et Industries 2002; 3: 3-14

[129] Arola D, Ramulu M. An Examination of the Effects from Surface Texture on the Strength of Fiber Reinforced Plastics. Journal of Composite Materials 1999; 33: 102-123 
[130] Agarwal BD, Broutman LJ, Chandrashekhara K. Analysis and performance of fiber composites, $3^{\text {rd }}$ ed. John Wiley \& Sons Inc. Hoboken, NJ. Hoboken, N.J.: John Wiley \& Sons Inc. Hoboken, NJ. 1990

[131] Akavci SS. Analysis of thick laminated composite plates on an elastic foundation with the use of various plate theories. Mechanics of Composite Materials 2005; 41

[132] Nettles AT. Basic mechanics of laminated composite plates, NASA reference publication No. 1351. National Aeronautics and Space Administration Marshall Space Flight Center MSFC, Alabama 35812. 1994: 10-16

[133] Torayca T700 preliminary technical data sheet No. CFA-006, Toray carbon fibers America, Inc. www.torayusa.com

[134] Hexcel, HexPly, M21 $356^{\circ} \mathrm{F} \quad\left(180^{\circ} \mathrm{C}\right)$ curing epoxy matrix, http://www.matweb.com/search/datasheet.

[135] Hüsnü Dirikolu M, Aktaş A. Analytical and finite element comparisons of stress intensity factors of composite materials. Composite Structures 2000; 50: 99-102

[136] Haque A, Ahmed L, Ramasetty A. Stress Concentrations and Notch Sensitivity in Woven Ceramic Matrix Composites Containing a Circular Hole-An Experimental, Analytical, and Finite Element Study. Proceedings of High Temperature Ceramic Matrix Composites 2005; 88: 2195-2201

[137] Ng SP, Lau KJ, Tse PC. 3D finite element analysis of tensile notched strength of $2 / 2$ twill weave fabric composites with drilled circular hole. Composites Part B: Engineering 2000; 31: $113-132$

[138] Choi M, Park J, Kang K, Kim W. Application of thermography to analysis of thermal stress in the NDT for compact tensile specimen 2006 
[139] Varvani-Farahani A, Haftchenari H, Panbechi M. A fatigue damage parameter for life assessment of off-axis unidirectional GRP composites. Journal of Composite Materials 2006; 40: $1659-1670$

[140] Davim JP, Rubio JC, Abrao AM. A novel approach based on digital image analysis to evaluate the delamination factor after drilling composite laminates. Composites Science and Technology July 2007; 67: 1939-1945

[141] Rakesh PK, Singh I, Kumar D. Failure prediction in glass fiber reinforced plastics laminates with drilled hole under uni-axial loading. Materials and Design 2010; 31: 3002-3007

[142] Arola D, Williams CL. Estimating the fatigue stress concentration factor of machined surfaces. International Journal of Fatigue 2002; 24: 923-930

[143] Ramulu M, Kunaporn S, Jenkins M, Hashish M, Hopkins J. Fatigue performance of highpressure waterjet-peened aluminum alloy. Journal of Pressure Vessel Technology, Transactions of the ASME 2002; 124: 118-123

[144] Taylor D, Clancy OM. The fatigue performance of machined surfaces. Fatigue \& Fracture of Engineering Materials \& Structures 1991; 14: 329-36

[145] Grzesik W, Rech J, Zak K, Claudin C. Machining performance of pearlitic-ferritic nodular cast iron with coated carbide and silicon nitride ceramic tools. International Journal of Machine Tools \& Manufacture 2009; 49: 125-33

[146] Naderi M, Kahirdeh A, Khonsari MM. Dissipated thermal energy and damage evolution of Glass/Epoxy using infrared thermography and acoustic emission. Composites Part B (Engineering) April 2012; 43: 1613-20

[147] Naderi M, Khonsari MM. On the role of damage energy in the fatigue degradation characterization of a composite laminate. Composites Part B: Engineering Feb. 2013; 45: 528-37 
[148] Wei B-, Johnson S, Haj-Ali R. A stochastic fatigue damage method for composite materials based on Markov chains and infrared thermography. International Journal of Fatigue 2010; 32: 350-360

[149] Novovic D, Dewes RC, Aspinwall DK, Voice W, Bowen P. The effect of machined topography and integrity on fatigue life. International Journal of Machine Tools \& Manufacture 2004; 44: 125-34

[150] Quaresimin. M. Fatigue of woven composite laminatesunder tensile and compressive loading 2002: 1 - 8

[151] Rahme P, Landon Y, Lagarrigue P, Lachaud F, Piquet R. Study into causes of damage to carbon epoxy composite material during the drilling process. International Journal of Machining and Machinability of Materials 2008; 3: 309-325

[152] Saleem M, Bougherara H, Toubal L, Cénac F, Zitoune R. Analysis of stresses in CFRP composite plates drilled with conventional and abrasive water jet machining. In: . Switzerland: Trans Tech Publications. 2013: 127-143

[153] Saleem M, Toubal L, Zitoune R, Bougherara H. Thermographic evaluation of CFRP specimens Drilled with conventional and abrasive water Jet techniques, The 19TH International Conference on Composite Materials, eProceedings, Montreal Canada July 28 to August 2, 2013 : 3785-3795

[154] Saleem M, Toubal L, Zitoune R, Bougherara H, Cénac F. Influence of experimental drilling process on the mechanical behaviour of CFRP composite plates, Presented in ECCM15 $15^{\text {TH }}$ European Conference on Composite Materials, Venice, Italy 24-28 June 2012,

[155] Saleem M, Sawi I. El, Zitoune R, Bougherara H. Influence of Conventional and NonConventional machining technique on the damage behavior of the CFRP Composite Assembly (under prepration) 
[156] Saleem M, Sawi I. El, Zitoune R, Bougherara H. Effect of conventional and abrasive water-jet machining technique on the stress life of qasi-isotropic carbon/epoxy composite specimens (under prepration) 